\title{
Resource-use and recursion by a mega-herbivore Elephas maximus borneensis
}

\author{
A thesis presented in fulfilment of the requirement \\ for the degree of \\ Doctor of Philosophy \\ in \\ Ecology and Biodiversity \\ at \\ Victoria University of Wellington
}

\section{Megan English \\ 2015}




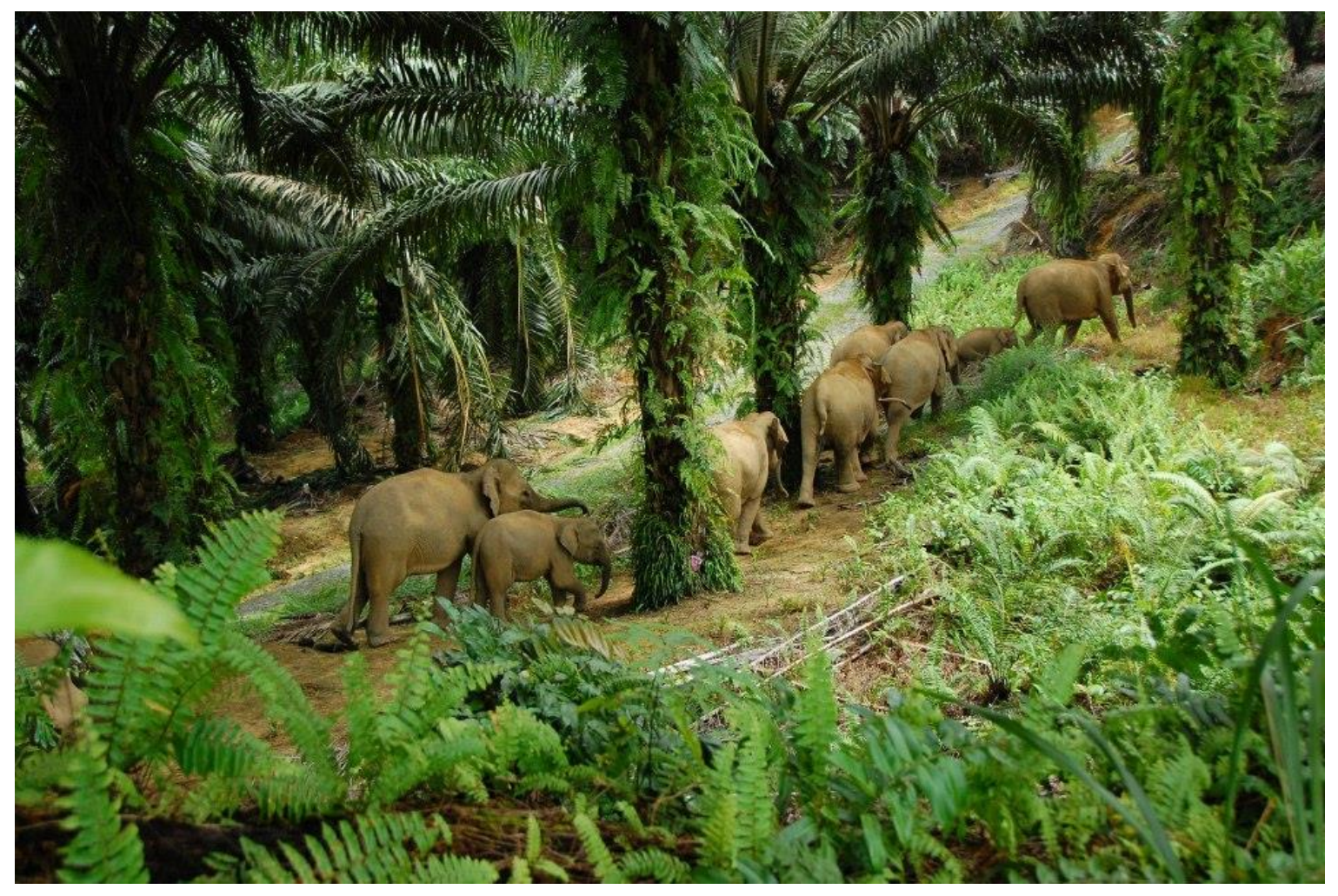

A herd of Bornean elephants (Elephas maximus borneensis) traverse an oil palm plantation between forest fragments, Sabah 2011.

Photo by Sulaiman Ismail 


\begin{abstract}
This thesis examines the ecology of elephants (Elephas maximus borneensis) that inhabit the Lower Kinabatangan region of Sabah, Malaysia. My research focused on improving our understanding of their habitat use and food-plant preference over varying spatial and temporal scales, and tested recursion hypotheses. Recursion, the reuse of sites or plants over time, has rarely been explored in wild animals. Studies of recursion promote understanding of species ecology as they explore temporal variation in resource-use. Recursion by herbivores may be a foraging strategy for optimising resource-use by returning to sites to coincide with plant recovery. A review of the recursion literature revealed that previous studies had not considered recursion that leads from foraging theory; this informed the research and the design of the chapters on recursion at two spatial scales - site and plant. The review also demonstrated the need to integrate the large amount of research on recursion-like processes with the new research topic of recursion. Such processes include site reuse associated with spatial memory, resource recovery and foraging site-fidelity. The scarcity of studies of these topics in large, wild herbivores was also evident.

I chose to investigate recursion ecology in the Bornean elephant because this provided an opportunity to test hypotheses for repeated resource-use, and to improve our understanding of resource ecology for a mega-herbivore. I expected recursion to occur less frequently in elephants compared with smaller herbivores. Mega-herbivores have a reduced requirement for high quality food. They should also consume more resources per visit, resulting in more time needed for resources to recover and therefore less frequent recursions. However, elephants have a more highly developed spatio-temporal memory than other herbivores and therefore may have greater ability to return more often to profitable foraging sites and plants.

To investigate elephant habitat use I first characterised the habitat types in the Lower Kinabatangan Wildlife Sanctuary (LKWS) based on their floristic composition and physical characteristics. Elephant habitat-use was measured using indirect observations of feed sign along ten $1 \mathrm{~km}$ long and $4 \mathrm{~m}$ wide strip-transects randomly placed throughout the LKWS. Elephants exhibited a strong preference for open grass areas along forest margins and avoided swamps and recently logged or cultivated habitat. Two of the most common habitats, lowland mixed
\end{abstract}


dipterocarp and semi-inundated forest, were neither selected nor avoided. The habitat types identified and their use by elephants underpinned the subsequent chapters on recursion and elephant food-plant preferences.

I investigated recursion to foraging sites using GPS collars to track the two main elephant herds in the LKWS in order to examine their behaviour and sample sites. Recursion was common, occurring at 48 of 87 foraging sites, within 48 hours and between 151-250 days. This indicated that elephant foraging strategies involve site sampling and timing of returns to coincide with some periodicity in site quality. I also found that recursion occurred if the site had previously been occupied for longer periods than sites receiving no recursions. The number of days that had passed between the first visit and recursion was also positively correlated with time spent at the recursion site. Sites that received most recursions were within the elephants' preferred habitat; that is, open grass areas along forest margins. These findings indicate that recursion occurs for the repeated exploitation of higher quality foraging sites and is perhaps timed for the recovery of their food plants.

The hypothesis that plant recovery rates influence recursion periodicity had not been tested previously in wild populations. The growth of new shoots on plants from 30 species that were previously selected for feeding by elephants were measured each month for 9 months, or until they were re-browsed by elephants, to learn if plant recovery rates influence recursion time. Recursion to grasses was found to coincide with full recovery but the elephants prematurely browsed other plant types. This suggests that elephant foraging strategies influence vegetation community structuring and may maintain or enhance grass patches. My results from Chapters 35 demonstrate recursion at two spatial scales, site and plant, and indicate that the elephants forage optimally in their preferred habitat.

It was also necessary to understand the proportion of grass and browse in the elephant diet and what influences the selection of browse species. Food-plant use and availability analyses found that contrary to what was expected of a generalist herbivore, plants were not selected by elephants in proportion to their availability. The finding that grasses form a major and highly preferred part of Bornean elephant diet was unexpected because they had been regarded as a forest-feeding species. One hundred and eighty-two plants were eaten and 185 plants from 18 species were measured for species availability along 12 transects. I identified a preference for 
grass rather than browse species despite grasses being less abundant, confirming the importance of grass and grass patches - two spatial scales - to elephants in the LKWS. Previous work has implicated the size and vigour of plants as important in herbivore food-plant selection. I found that elephant browse preferences were not influenced by plant vigour and plant size. Bornean elephant foraging strategies are therefore primarily focussed on the optimum harvesting of grass in discrete patches within the forest and the riparian zone.

To manage this population of elephants it is important to understand their relationship with their food resources. My study has contributed in this regard in ways that are of general importance to understanding elephants and other mega-herbivores, as well as in ways that are of specific importance to the conservation of Bornean elephants in the LKWS. Firstly, in order to meet the elephant population's dietary needs, access to open grass areas must be maintained. Forest rehabilitation projects should incorporate these areas as part of their landscape management strategy. For example, corridors should be designed and placed to assist the movement of elephants amongst preferred grassy foraging sites.

Secondly, this study provides scientific support for effective ways to identify profitable foraging areas for wild herbivores across a variety of landscapes. Studies of recursion and the characters of sites and plants that receive repeated visits provide more direct and rapid ways of identifying what is important to herbivore populations.

Moreover, if the elephant, the largest land mammal and herbivore, is so elaborately and concurrently recursive at different spatial and temporal scales, then recursion is likely to be widespread amongst other herbivores. I therefore recommend further examination of recursion behaviour across a wider range of wild herbivores. Advances in our understanding of herbivore ecology and our ability to conserve and manage wildlife habitat will require a direct refocus on repeated resource-use and will depend on redesigning studies to consider the scales at which recursion occurs. 


\section{Acknowledgements}

In particular I thank my supervisor Wayne Linklater for his advice, encouragement and support throughout the last 4 years. He has always responded thoughtfully and patiently to my enquiries and has done his best to guide me towards clear solutions. Thanks to other students and staff at Victoria University of Wellington who have been good friends or offered advice, including Roan Plotz, Rosalyn Anderson-Lederer, Andrew Stringer, Gaius Wilson, Phil Lester, Phil GarnockJones and Dalice Sim. Thank you also to administrative staff Mary Murray, Sandra Taylor, Paul Marsden, Patricia Stein and Mark Stephen. The PhD scholarship and completion scholarship awarded by VUW was much appreciated.

Graeme Gillespie, who was the Director of Conservation at Zoos Victoria, encouraged me to apply for support from his institution, which generously funded the project. I am also grateful to Chris Banks, who is the Manager of International Conservation Partnerships at the zoo.

Graeme, who became my co-supervisor, encouraged me to consider a variety of options when it came to statistical analyses and other aspects of method. This assisted me to refine my analyses, consider all options and justify my decisions. Thanks also to my second co-supervisor, Benoit Goossens, for his ongoing advice and for his help in my obtaining a research permit for Sabah. Benoit and another of his PhD students, Nurzhafarina Othman, from Cardiff University and Danau Girang Field Centre, allowed me to access the GPS data for the elephants collared by them. Other students at the field centre, in particular Danica Stark and Andrew Hearn, were also willing to help when needed.

Although they were not directly involved with this study, I must thank Professors Lesley Rogers and Gisela Kaplan from the University of New England. They supervised my earlier Honours research on zoo elephants and have offered encouragement and excellent advice throughout the $\mathrm{PhD}$ process.

While based in Sukau, a small village in the Lower Kinabatangan region where I did my fieldwork, I worked with staff from the non-government organisation Hutan Kinabatangan Orang-utan Conservation Project, which also has an Elephant Conservation Unit. The Hutan directors Marc Ancrenaz and Isabelle Lackman allowed me to use their staff as field assistants. People like them, who work at the grass roots level of conservation and face countless obstacles 
in their work but charge on relentlessly to protect species like the orang-utans, the elephants and their habitats, have been inspirational. I was lucky to be able to collaborate with them.

Thank you to the Sabah Wildlife Department, in particular my counterpart Sen Nathan and the director Laurentius Ambu, for allowing me to undertake research on the Bornean elephant; and to the Economic Planning Unit, Kuala Lumpur, which readily approved and extended my research permits.

Without my research assistants I could not have completed the fieldwork. The six staff from the Elephant Conservation Unit helped me to locate and track the elephants and I thank them very much, in particular Sulaiman Ismail. On several occasions when we were confronted with danger he would hoist me up trees when we had overly curious elephants to deal with, deter poachers from our campsite at night, chase off wild boar and monitor lizards from our food supply, and keep an eagle-eye on our surroundings for crocodiles and snakes when we tracked the elephants through the swamps. He also got me to the clinic when I was badly stung by hornets and again to the hospital a few hours away when I contracted what the doctors diagnosed after many blood tests as 'jungle fever', an illness that brought on fever and rapid weight loss (later discovered to be caused by a tick bite). I also thank Mardiana Ismail, Sulaiman's sister. She joined us on our camping expeditions and helped to prepare food including such things as giant river prawns, salted green mangoes and sambal after a long day in the forest. One of my favourite memories is sitting up until 3 am with the two of them and hand-sewing our tents back into one piece after a surprise visit from a sub-adult male elephant. We had been gathering data elsewhere and as we arrived back at camp we were just in time to see him disappear into the forest with a pillow under his trunk and a tent over his back. I found my passport in a pool of elephant urine, an explanation that Australian immigration bemusedly accepted as an explanation for the fragrant and faded yellow pages when I returned to Australia a few weeks later.

The parents of Sulaiman and Mardiana would often come and visit us in the forest. Their father, Ismail, a local fisherman, would share his catch of the day, grilled over an open fire along with the delicious kampung nasi goreng made by their mother, Asiah. I used to sit late into the night in my hammock enjoying the animated stories shared by this family about the forest and the elephants and Sungai culture. 
Lastly I would like to thank my family. My parents, Linda and Tony, have travelled and worked in some of the most remote places in the world, easily adapting to and appreciating different cultures. This upbringing ensured my brother Cain and I grew up open-minded and with a sense of adventure, taking advantage of any excuse to be out of our comfort zone. They, as well as Cain and his wife Yvonne, have encouraged and supported me throughout this journey in many ways. I already knew how lucky I was with the card I was dealt with my family, but this extended time apart and their unconditional support throughout has made me appreciate them more than I already did.

I dedicate this thesis to Mum and Dad and also to my late Poppa, Len. He taught me to believe in myself and encouraged me to achieve anything I set my heart on. He and I had a saying, from when I was about 4 years old (while we posed together like Auguste Rodin's 'The Thinker'), "This causes for careful deliberation!" This saying frequently came to mind during the last four years and put a smile on my face whenever I faced a challenge. 


\section{Disclaimer}

The present dissertation includes two paper manuscripts accepted for publication in scientific peer-reviewed journals. This results in some duplication in the description of methods for Chapters Five and Six and in the Introductions on pages 42 and 74. The submitted manuscripts include other authors with me as lead author in both cases. For each paper my input was greatest. I designed the research, undertook the field work, analysed the data and wrote the manuscripts. I was, however, assisted by my co-authors. I hereby declare all the work to be my own and that I have acknowledged all those that helped me and contributed in producing this dissertation.

Megan English 


\section{Prologue}

In 1981 I was born in Kandy, Sri Lanka, to Australian parents who were working there. We lived there for only a few years before moving to the Philippines. Although my memory of that time in Kandy is meagre, I grew up with my parents' photographs of the large tusker kept at the Temple of the Tooth in Kandy, and the elephants that would bathe daily with their mahouts in the Mahaweli River a few hundred metres from our house. As I got older and moved from one country to another, I became aware of the increasing threats to elephants in the wild and the welfare problems facing those in captivity. At high-school in Australia, and later at university, my scientific interest in elephant behaviour and conservation grew.

Early in 2002 I began my undergraduate degree at LaTrobe University in Melbourne. I completed a Bachelor of Animal Science majoring in Zoology, after which I was offered a place for an Honours year. I decided that before undertaking further study I would go back to Sri Lanka to volunteer with elephants. As a volunteer, I collected behavioural data on two elderly female elephants for scientists at the University of Peradeniya.

When I enquired at LaTrobe about the topic of my Honours, I was unable to find a supervisor willing to supervise a study on elephants so I looked at other universities in Australia and overseas. Many years earlier, when I was at high school, I had completed work experience with Professors Lesley Rogers and Gisela Kaplan at the University of New England. They were eager to supervise my Honours research on elephants, so I enrolled at UNE, where I studied the influence of enrichment activities on the behaviour of elephants at Melbourne Zoo (English et al. 2014a).

After completing my Honours year with a good result I travelled throughout Asia, considering my options for a $\mathrm{PhD}$ in elephant behaviour and ecology. My original plan was to study the Sri Lankan elephant but due to the escalating conflict associated with the late stages of the country's civil war I was advised against doing fieldwork there. Later that year I travelled to Borneo, eager to learn about the elusive Bornean elephant. There I met with conservationists to discuss research prospects on this under-studied species. It was clear that in order to manage the population of elephants that live in a highly degraded and fragmented landscape it was of particular importance to understand their relationship with their food resources. I approached Wayne Linklater at Victoria University of Wellington to be my supervisor. He agreed, and this thesis is the outcome. It is my hope that my work will benefit elephant conservation in Borneo by providing information on their resource-use that can inform policy makers of suitable landscape management strategies within the Lower 
Kinabatangan Wildlife sanctuary. I also hope that this study contributes to developing techniques for applying foraging theory to wild large herbivore foraging strategies. 


\section{Table of Contents}

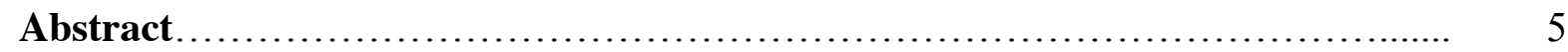

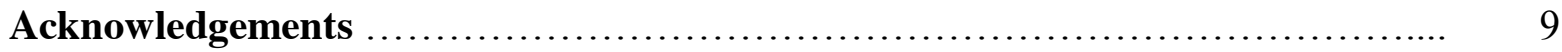

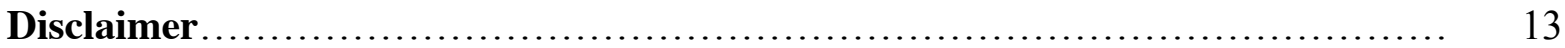

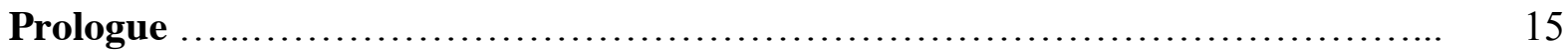

Chapter 1 Introduction ................................................. 19

Optimal foraging by herbivores ......................................... 20

Mega-herbivore food-plant relationships.................................... 22

Recursion ................................................................ 24

Conservation implications and the future of recursion studies...................... 25

Focal species............................................................ 26

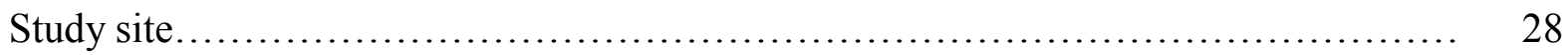

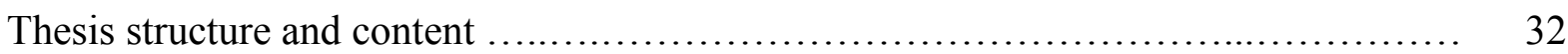

Chapter 2 Recursion and its application to foraging by a mega-herbivore ............. 35

Optimal foraging and recursion............................................ 37

Previous studies of recursion .................................................... 40

Recursion at two scales: site and plant....................................... 44

Recursion by elephants.................................................... 44

Body size.................................................................. 44

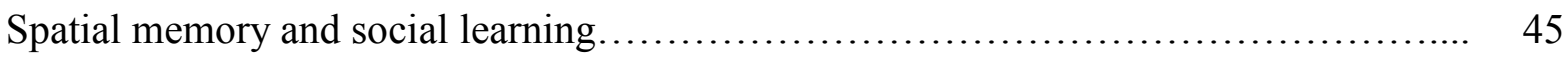

Technological advances facilitate studies of recursion............................ 47

Recursion, ecology and conservation......................................... 48

Chapter 3 Habitat classification, availability and use by elephants in the Lower

Kinabatangan Wildlife Sanctuary............................................ 51

Introduction................................................................. 53

Methods................................................................. 55

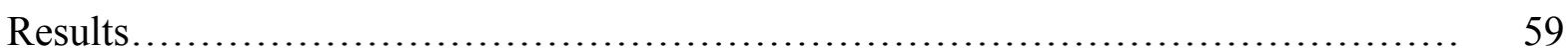

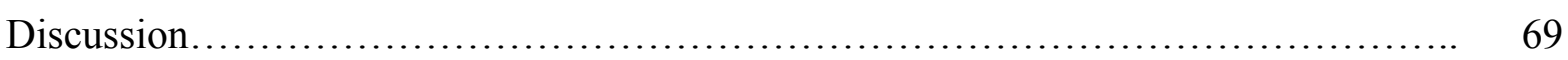

Chapter 4 Foraging site recursion by forest elephants ......................... 73

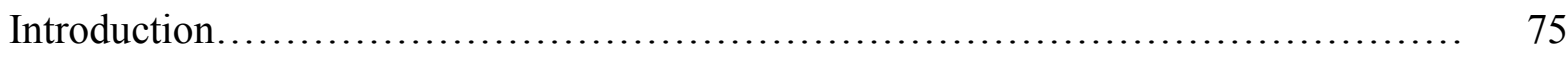

Methods................................................................. 77

Results............................................................... 82

Discussion.............................................................. 86 
Chapter 5 Recursion to browsed plants ....................................... 91

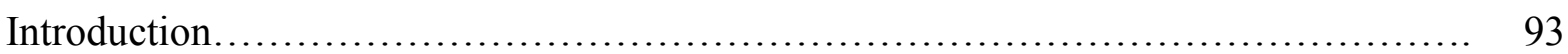

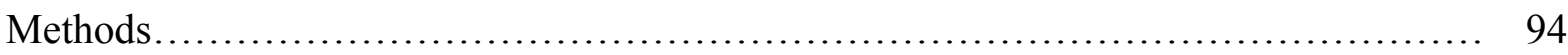

Results.................................................................. 97

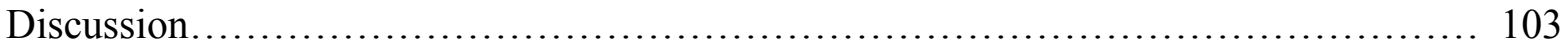

Chapter 6 Plant selection and avoidance by the Bornean elephant in tropical forest: does plant recovery rate after herbivory influence food choices? ......................... 107

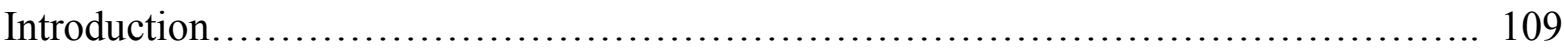

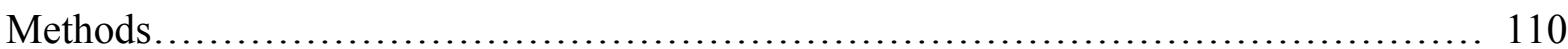

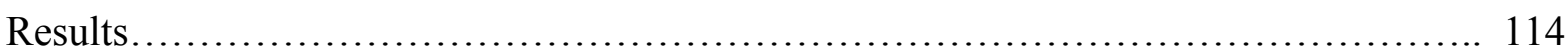

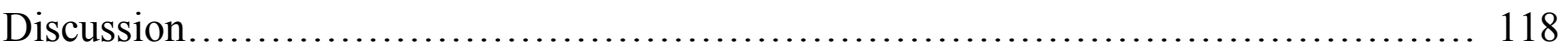

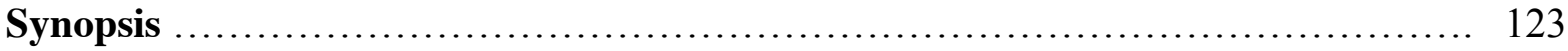

Appendix One Distance across elephant herd while foraging ...................... 131

Appendix Two Comparison of recursion data between two focal female elephants ..... 133

Appendix Three Preliminary analyses of elephant movement using a HMM............ 135

Appendix Four PDF published paper (Chapter Four) .......................... 141

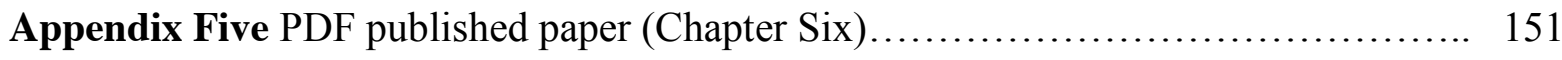

Appendix Six Electronic file literature from review chapter............... Electronic file

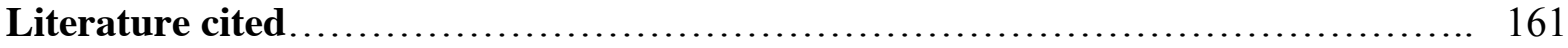




\title{
Chapter One
}

\author{
Introduction
}

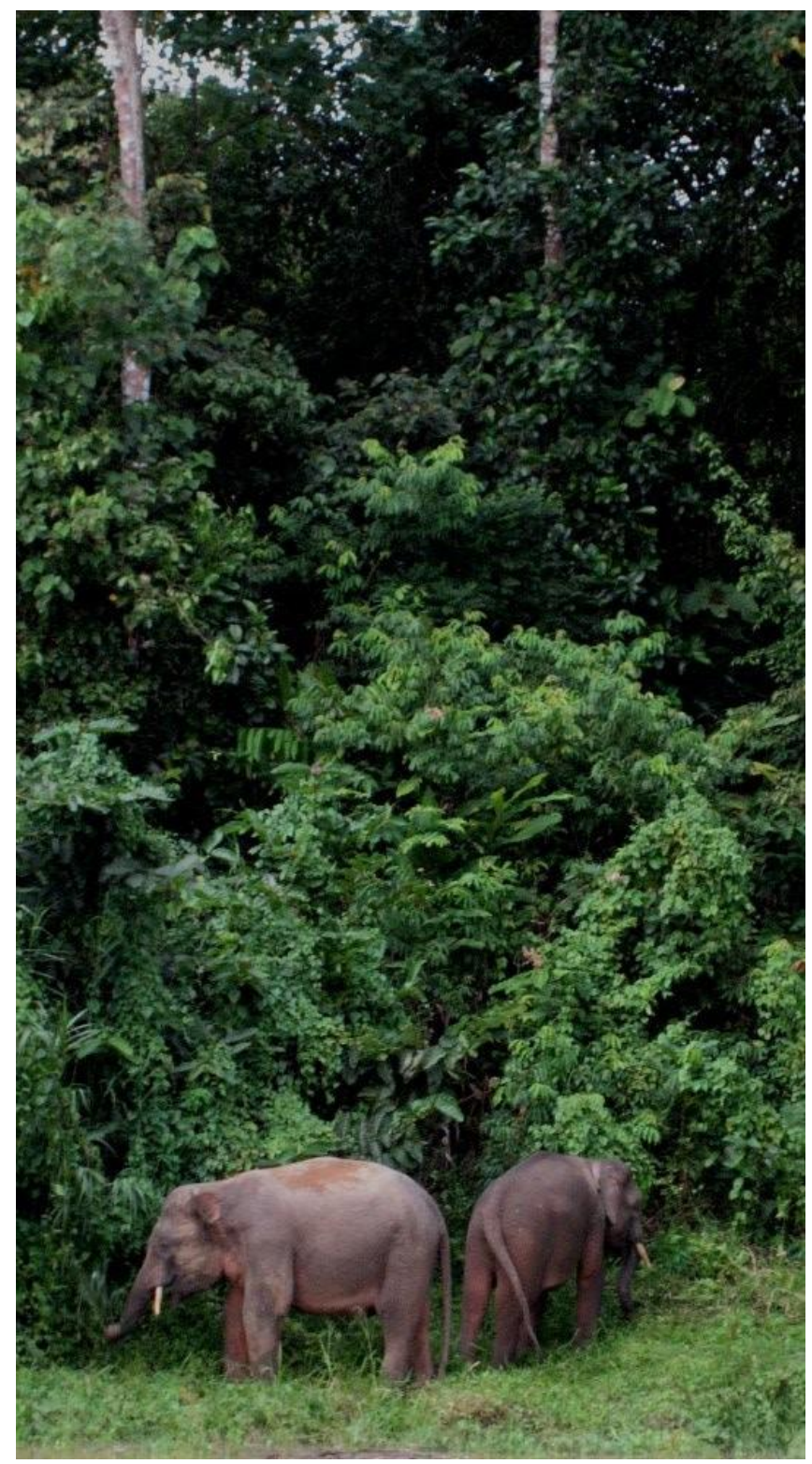

Juvenile male elephants foraging in riverine ecotone habitat

Lower Kinabatangan Wildlife Sanctuary, Sabah, 2011

Photo by Tony English 


\section{Introduction}

A persistent challenge to ecological science and for wildlife conservationists is to find ways of maintaining viable populations of species and habitat (Soulé, 1985). In order to accomplish this, an understanding of species population distribution and abundance (Kunin, 1998) and resource-use is essential (Kertson \& Marzluff, 2011). Resource-use will vary depending on an animal's intrinsic requirements for growth, survival, and reproduction (White, 1983; McNamara \& Houston, 1996). Resource quantity and quality, including resource regeneration rates, and also the costs to the animal associated with finding and exploiting resources will influence how resources are utilised (Charnov, 1976; White, 1983). Depending on these influences, resource-use by herbivores is expected to vary over different spatial and temporal scales. In order to describe the foraging strategy of a species and the ecological rules it follows, we need to know how preferences change and are satisfied over these scales. Together, an understanding of animal preferences and their variation through space and time provide a basis for determining species habitat and spatial requirements, which are the cornerstone of species conservation.

\section{Optimal foraging and herbivores}

Evolution by natural selection should mean that herbivores trend towards optimal foraging. Thus resources with reinforcing properties are selected and those that impose a cost or lack benefits are avoided. Selection:avoidance or use:availability ratios reveal if animals feed or use certain resources randomly or selectively (Johnson, 1980). If resources are selected in proportion to their availability then this suggests a generalist or random strategy. However, if resources are used out of proportion, more or less, than their availability this indicates either a preference or avoidance, respectively, of those resources.

Resource abundance and availability vary at different spatial and temporal scales, and travel time and distance to obtain these resources are a cost to an animal. Herbivore foodplant relationships, therefore, are significantly influenced by trade-offs between the advantage of consuming a given diet and the cost of searching for it (Stephens \& Krebs, 1986). Two commonly referred to theories associated with a cost-benefit trade-off in the consumption of a resource are giving up densities (GUD) and ideal free distribution (IFD) (Fretwell \& Lucas, 1970; Charnov, 1976). The theory of GUD predicts that resources are utilised until the cost of utilising them further is greater than the cost of seeking resources 
elsewhere. IFD predicts that the density of animals utilising an area is a reflection of the resources available. For example, more abundant resources support a higher density of animals and less abundant resources support a lower density (Charnov, 1976).

Animal resource preferences also depend on variations in the animals' morphology and physiological function (Provenza et al., 2003). For example, depending on their body size, utilisation might depend on plant nutritive value or plant morphology and availability (Nicotri, 1980). Smaller animals require feed of higher nutritive value than larger animals and are generally more selective (Jarman, 1974). Large herbivores, with their lower energy requirements per unit body mass, have greater gut capacity for ingesta retention, facilitating a higher tolerance for lower quality (high fibre content) forage (Parra, 1978; Demment \& van Soest, 1985; Illius \& Gordon, 1990). Larger herbivores, therefore, tend to ingest a diet lower in quality than smaller herbivores, and favour quantity over quality. For larger herbivores, the benefits of ingesting abundant forage resources outweigh the costs of searching for forage of higher nutritional return which is usually rare or more heterogeneously distributed in the environment. As a result small herbivores are generally more selective feeders than large herbivores (Demment \& van Soest, 1985; Owen-Smith, 1988). The selection of highly nutritious food items by smaller animals is also a reflection of their higher metabolic rates. For instance, if all animals could digest similar quantities of food relative to body weight, then higher metabolic rates would require smaller animals to seek higher quality food than larger animals (Jarman, 1974).

High quality plant parts usually form only a small proportion of a plant. At the plant community scale there is generally not as much high quality as low quality plant material available to herbivores. It is to be expected, therefore, that larger herbivores requiring a large absolute intake of food, should use low quality food which is available in larger quantities whereas smaller herbivores select higher quality food in smaller quantities (Bell, 1971; Jarman, 1974). Among mammalian herbivores, selective feeding has been expressed mainly in terms of plant parts consumed, rather than the species diversity of the diet. However, there is considerable evidence that large herbivores also exhibit a degree of non-random selective foraging and track areas of highest quality forage (Sinclair, 1977; McNaughton, 1985; Fryxell et al., 1988, 2005; Owen-Smith, 2012). For example, high quality 'lawns' form where grazing is concentrated at localised sites where typically short sward grasses are maintained in early growth stages by herbivores that facilitate new growth (McNaughton, 1984; Archibald, 2008; Cromsigt \& Olff, 2008). Similarly, browsing on woody plants that respond with tolerance traits (Fornoni, 2011) associated with increased palatability attracts further 
browsing (Cromsigt \& Kuijper, 2011). These feeding areas are referred to as 'grazing' and 'browsing' lawns, respectively, and a possible mechanism for the creation and preservation of such patterns is the positive feedback that occurs between grazing/browsing and plant palatability (Mouissie et al., 2008).

Plant growth rate is also expected to influence herbivory. Price (1991) proposed a hypothesis that any plant module, individual, or species, that grows rapidly and ultimately reaches a large size, relative to the mean growth rate and size of the population of modules, individual plants or plant species, suffer enhanced probability of herbivore attack due to these often having a higher nutrient content and less toxic compounds for herbivore defence. Furthermore, forage quality can be improved by regular herbivory due to increased nutrient cycling within foraging sites. This can increase the nutritional content of new growth (Jameson, 1963; Everson, 1966; Kamstra, 1973; Ydenberg \& Prins, 1981; Coppock et al., 1983; McNaughton, 1985 and DuToit et al., 1990) or increase the availability of green nutritious material (Prins et al, 1980; Coppock et al., 1983) but herbivory may also lead to progressive depletion of resources (McNaughton, 1984).

In conjunction with variations in spatial and temporal distribution of resources and their growth forms, animal morphology and physiology, and the risk of resource depletion, optimal foraging is a challenge for many herbivores (De Vries et al., 1989). Herbivore foraging efficiency would increase if information on variations in resource quality and quantity were retained, or anticipated, and incorporated into a foraging strategy to optimise the cost-benefit balance and to minimise over-utilisation of available resources. The cognitive capacity of different animal species for this ability, however, also varies. Larger herbivore are, generally speaking, better equipped for processing and interpreting environmental information and implementing strategies to benefit from it (Hart et al., 2008). In this regard, therefore, the world largest herbivores are interesting case-studies in resource-use ecology.

\section{Mega-herbivore food-plant relationships}

Owen-Smith (1988) came up with the term 'mega-herbivore' to describe very large herbivores with an adult bodyweight greater than $1000 \mathrm{~kg}$. He noted that large body size means mega-herbivores are largely immune to non-human predation while their bulk feeding allows them to tolerate food of a lower quality than that required by smaller herbivores. In addition to larger species being less selective in their dietary choice than smaller species, due to lower nutrient requirements per unit of body mass (Jarman, 1974), Bell (1971) pointed out 
that non-ruminants should tolerate even lower dietary quality than ruminants of similar body mass. As hindgut fermenters, their faster digestive passage compensates for lowered digestive efficiency on fibrous diets (see also Janis, 1976). Hindgut fermenters are disproportionately represented in the mega-herbivore class, for example elephants and rhinoceros (Owen-Smith, 1988).

Because of the requirement for large quantities of lower quality food, mega-herbivore activity can have a major influence on ecosystem structure and function, primarily on vegetation. For example, elephants are a large social species that forages and moves across the landscape in large herds (Laws, 1970; Barratt \& Hall-Martin, 1991; Huntly, 1991; Mapaure \& Campbell, 2002; Conybeare, 2004; Staub et al., 2013). While other herbivores also influence ecosystem structure, the daily amount of vegetation consumed per elephant is greater than by other herbivores (Van Aarde \& Jackson, 2007). For this reason megaherbivores, such as elephants, need to cover a vast area in order to meet their food requirements, a feat that is becoming increasingly difficult owing to anthropogenic reductions in, and restrictions to, elephant habitat, especially from land development for agriculture.

Mega-herbivores are often referred to as 'ecosystem engineers' because they can modify the relative abundance, architecture, physiology, biochemistry, productivity and phenology of plants they feed on and are therefore agents of habitat change (Laws, 1970; Bryant, 1981; Bergström \& Danell, 1987; du Toit et al., 1990; Ben-Shahar, 1993; Prins \& Olff, 1998). Such modifications can increase the presence of chemical or physical deterrents in plants, reducing subsequent herbivory (Bryant, 1981; Karban \& Meyers, 1989; Hulbert \& Anderson, 2001). More often, however, herbivory leads to increased palatability and therefore probability for repeated foraging (Bell, 1971; McNaughton, 1976, 1984; Danell et al., 1985; du Toit et al., 1990; Duncan et al., 1998; Bergström et al., 2000). This can lead to a positive consumer-resource loop, such as the maintenance of grazing lawns, a process that is particularly well-studied in ungulate species (McNaughton, 1984) but not so with megaherbivores such as the elephant.

Elephants are ecosystem engineers because they have complex, scale-dependent effects on habitat structure (Bond, 1993; Jones et al., 1996). In Africa, elephant induced changes in the structure and composition of vegetation have been studied (Ben-Shahar, 1993) and found their impact on woody vegetation has led to a decrease in numbers of trees and an increase of open areas (Conybeare, 2004; O’Connor et al., 2007). High elephant abundance, however, is not always associated with low woody cover (Chamaillé-Jammes et al., 2009) but changes in canopy cover resulting from the uprooting and breaking of trees and shrubs by 
elephants can influence the species composition and structure of the herbaceous layer (Belsky \& Canham, 1994). Elephant resource-use may also indirectly impact other herbivores. For example, in Africa trends in elephant populations are often correlated with opposite trends in browser populations (Valeix et al., 2008). On the other hand, studies also show that elephants may facilitate smaller browsers by increasing food availability (Rutina et al., 2005; Makhabu et al., 2006a; Pringle, 2008).

\section{Recursion}

The importance of spatial and temporal variation in mega-herbivore resource-use is crucial for understanding the relationship of these ecosystem engineers within habitats and plant communities (Hastings et al., 2007). A study of recursion - the reuse of resources over varying temporal scales - is an approach that can be used to understand this relationship and its influence at different spatial scales.

Foraging recursion by herbivores includes re-browsing plants or re-visiting foraging sites. Previous herbivore foraging and resource-use studies have focused on the spatial and relatively short-term temporal selection of resources (Johnson, 1980; Thomas \& Taylor, 1990). This approach may result in failure to adequately consider the repeated use of resources that are of importance to the species. Understanding recursion patterns is fundamental to recognising the factors that govern home-range use and placement as these are a product of how an animal adjusts to environmental and biological heterogeneity in space and time (Patterson et al., 2008; Bar-David et al., 2009; Benhamou \& Riotte-Lambert, 2012; Riotte-Lambert et al., 2013).

Studies of recursion are needed in order to explore the spatial and temporal variation in animal resource-use. In natural environments resource availability varies in distribution, abundance and density through time and animals may re-use some resources and not others. For example, a rare plant or site may appear to be avoided compared to others but might be the subject of repeated use over a long time frame such that recursion studies of appropriate temporal scale may reveal high selection. Additionally, common plants or other sites may appear to be highly selected when utilised but are not subject to recursion and so are actually seldom used or even avoided longer-term. Thus, temporal studies of recursion are necessary to elaborate on spatial variation in availability and selection when assessing food and habitat preferences.

'Recursion' is a relatively new term in investigations of temporal variation in resource use. Nevertheless, there are a large number of studies of behaviour resembling recursion such as 
those that explore the influence of spatial memory and foraging (e.g. Thomson 1996, Watts, 1998; Garrison \& Gass, 1999), resource recovery and foraging (e.g. Davies \& Houston, 1981; Bell, 1990; Williams and Thomson, 1998) or foraging site-fidelity (e.g. Switzer, 1997;

Barraquand \& Benhamou, 2008). These concepts are relevant to studies of recursion but have not been applied in this way yet.

Studies of recursion by large herbivores in uncontrolled environments, for example free-ranging animals, are remarkably few but one study explored the frequency of site reuse over varying temporal scales (Bar-David et al., 2009) and another the influence of residency times on recursion (Riotte-Lambert et al., 2013). These studies, however, have not incorporated foraging theory by investigating the influence of site characteristics and resource recovery on recursion. The influence of resource recovery on recursion has been suggested as the next step (Bar-David et al., 2009) but until now it has not been explored in wild animals.

It is clear that a study of recursion in a mega-herbivore, like the elephant, could make a useful contribution to our understanding in ecology. Tests for recursion in a large freeranging animal that integrate like concepts and knowledge, might make advances in our understanding of resource-use at multiple spatial and temporal scales concurrently.

\section{Conservation implications $\&$ the future of recursion studies}

Recognising profitable areas and resources, and the ways these are exploited, can inform and foster development of better strategies for conservation and management of herbivore populations and their landscapes. According to Sanderson et al. (2002) effective conservation planning must include consideration of the heterogenous and dynamic nature of ecosystems (Huston, 1994; Pickett \& Rogers, 1997; White \& Harrod, 1997; Koehler, 2000). Nature is not homogenous, and most organisms, at one scale or another, depend on heterogeneity for their survival (Pulliam \& Danielson, 1991; Hanson et al., 1995; Tilman \& Kareiva, 1997). Animals rarely use the landscape homogenously, instead tending to cluster their activities among resource-rich areas (Charnov, 1976; Holling, 1996). Some species, like elephants, that use multiple habitats, depend on temporary concentrations of resources, move through the landscape in non-random ways depending on the spatial and temporal distribution of these resources (Kozakiewicz, 1995; Kinaird et al., 1996; Lima \& Zollner, 1996). Therefore, effective conservation planning should address species-specific resource needs at different spatial and temporal scales. Studies of recursion can address the influence of environmental 
and biological heterogeneity by exploring species resource-use at varying scales. This information can contribute to our understanding of the habitat requirements of herbivores, the factors determining their space-use patterns, and their food-plant relationships.

Ongoing advances in tracking technology allow scientists to explore animal resourceuse in greater detail by tracking animal movements over varying scales. In studies of recursion, this technology enables recording of the frequency of returns to sites (Bar-David et al., 2009) and the time elapsed between returns and residency times (Riotte-Lambert et al., 2013). Combined with on-ground measurements of biological and physical variables, evaluations of the environmental attributes underpinning high and low use areas over varying spatial and temporal scales may contribute to improved understanding of resource-use and foraging strategies.

Previous elephant resource-use and habitat selection studies have assisted conservation planning by identifying the influences of habitat fragmentation and conversion on elephant populations (Leimgruber et al., 2003). Elephant resource-use has also been explored to assist understanding the behavioural patterns associated with human-elephant conflict (Sukumar, 1990; Osborn et al., 2004). This has contributed to understanding the cause and timing of crop-raids in order to help design locally appropriate mitigation and management strategies (Webber et al., 2011).

\section{Focal species}

The Asian elephant (Elephas maximus) and its four sub-species (Sri Lankan Elephas maximus maximus, Sumatran Elephas maximus sumatranus, Bornean Elephas maximus borneensis and Indian Elephas maximus indicus), and the two African elephant species (savannah Loxondonta Africana and forest Loxondonta cycotis), are a fraction of the 175 recognised species in the order Proboscidea, many of which became extinct in the Quaternary glaciation (Shoshani, \& Tassy, 2005). A fifth sub-species of Asian elephant, the Javan (Elephas maximus sondaicus) has been extinct in Java since the late $18^{\text {th }}$ century. All Asian elephant species are endangered but the Sumatran is critically endangered (Sitompul, 2011). African elephants are listed as vulnerable and their numbers are decreasing in many parts of Africa due to illegal poaching as a result of the ivory trade as well as from habitat degradation and human encroachment (IUCN Redlist, 2014). The main threat to Asian elephants and the focal species of this study, the Bornean elephant, is habitat degradation and conversion of land from forest to agriculture and the increased human-elephant conflict that 
subsequently results. In Borneo these are associated mainly with the conversion of forest to oil palm plantations (Elaeis guineensis) (Ambu \& Andau, 2002; Othman et al., 2013).

The Bornean elephant is smaller than other Asian elephants and has larger ears, straighter tusks and a longer tail. It is found in the eastern and central parts of Sabah as well as the extreme north of the Indonesian province of Kalimantan. There are believed to be five main concentrations of Bornean elephants (Alfred \& Ahmad, 2010), totalling fewer than 2000 individuals: in Tabin Wildlife Reserve (140 601 ha); Lower Kinabatangan Wildlife Sanctuary (58 809 ha and the location of my study); Deramakot, Ulu Segama and Kalabakan Forest Reserves in Sabah (1 080529 ha); and Ulu Sembakung Nature Reserve in Kalimantan (79 408 ha) (Figure 1).

It is unclear whether the Bornean elephant is native to the island. Fernando et al. (2003) claim that it is a separate evolutionary unit and that independent evolution has occurred for 300000 years. However, the presence of this sub-species in Borneo for that period has been questioned because there are no authenticated finds of Asian elephant in any excavation in Borneo. Excavations in Borneo at Niah cave (Sarawak) and at Madai cave (Sabah) did not reveal elephant fossils despite evidence of other large Perissodactyl ungulates such as Rhinocerus sondaicus, Dicerorhinus sumatrensis and Tapirus indicus.

Although the Bornean elephant does not have a recent history of domestication, other than being housed in zoos in three countries (Lok Kawi Wildlife Park in Sabah, Portland Zoo in America and in unknown Zoos in China), another possibility is that it is descended from imported captive elephants that originated in Java and were released in northeast Borneo in the $18^{\text {th }}$ century (Cranbrook et al., 2008). This suggests that the Bornean elephant is, in fact, an introduced species. If it is descended from the now extinct Javan elephant it should be a conservation priority to protect this rare sub-species and its habitat and provide the potential for reintroduction to Java.

The elephants in the Lower Kinabatangan Wildlife Sanctuary, where this study took place, are restricted to linear forest bordered by the river and oil palm plantations. There are no corridors linking this population to other populations or to other suitable habitats. The closest being $>100 \mathrm{~km}$ to the southeast (Tabin Wildlife Reserve) and $>100 \mathrm{~km}$ southwest (Deramakot Forest Reserve) (Figure 1). Human-elephant conflict in this area is a concern as the elephants frequently enter oil palm plantations that have encroached on their home range (Estes et al., 2012; Othman et al., 2013). As well as an increase in the area being used for oil palm cultivation, conflict is also likely to be associated with the growing population of humans in the Kinabatangan area (from 45746 people in 1990 to 86783 people in 2000) 
(Sabah Department of Statistics, 2001) and the growing number of elephants. The elephant population in LKWS has been estimated as > 200 (published estimate: 298, 95\% CI: 152581) (Alfred \& Ahmad, 2010). It is believed the number of elephants has doubled in the last ten years (Ancrenaz pers. comm.).

So far, there have been only 11 published manuscripts specifically on the Bornean elephant. They include studies of their origin and genetics (Fernando et al., 2003; Cranbrook et al., 2008; Sharma et al., 2012), morphometrics (Othman et al., 2008), utilisation of a particular tree species (Matsubayashi et al., 2006), density and population estimation (Alfred \& Ahmad, 2010), home-range behaviour (Alfred et al., 2012), the influence of forest fragmentation on elephant movement (Estes et al. 2012), parasite prevalence (Hing et al., 2013), conservation (Payne et al., 2013) and human-elephant conflict (Othman et al., 2013). A thorough understanding of their resource-use and foraging ecology remains to be understood in order to guide conservation planning for the species within this fragmented and highly cultivated landscape.

\section{Study site}

\section{Location, climate and landscape}

Sabah, one of the 13 states in the federation of Malaysia, is located between the latitudes $4^{\circ} 8^{\prime}$ and $7^{\circ} 22^{\prime}$ north of the equator on the North-Eastern tip of Borneo. It covers about 76000 $\mathrm{km}^{2}$ in the northern portion of the island. The Lower Kinabatangan Region refers to the lower catchment of the Kinabatangan River including its floodplain and tributaries. The region lies between longitudes $117^{\circ} 90^{\prime}$ and $118^{\circ} 30^{\prime} \mathrm{E}$ and latitudes $5^{\circ} 50^{\prime}$ and $5^{\circ} 10 \mathrm{~N}$. This area represents a major portion of the eastern lowlands of Sabah and includes about 65000 ha of wetlands and 330000 ha of flood-free land (Hai et al., 2001). The dominant landform of the Lower Kinabatangan region is the extensive floodplain and its swamps. Soils are predominantly alluvial and derived from sedimentary deposits often rich in magnesium. Beyond the floodplains, soils are derived from sedimentary rocks (Azmi, 1998). The area is relatively flat with occasional limestone outcrops. Limestone areas are of late-Miocene age and are comprised of detrital and coral limestone.

The Kinabatangan floodplain is characterized by a warm, wet and humid tropical climate. The larger temperature variations are diurnal rather than seasonal. Mean monthly temperatures range between $21^{\circ} \mathrm{C}$ and $34^{\circ} \mathrm{C}$ (Ancrenaz et al., 2004). The north-easterly 
monsoon brings high monthly rainfall from October to February, although rainfall is also common from March to September. Dry months, with mean monthly rainfall $<60 \mathrm{~mm}$ tend to occur at roughly 3-year intervals. The mean annual rainfall is $3000 \mathrm{~mm}$ (Acres \& Folland, 1975). The Kinabatangan River, at $560 \mathrm{~km}$ long, is the second longest river in Malaysia and has a total catchment area of about $16800 \mathrm{~km}^{2}$. The river flows in a north-easterly direction to the Sulu Sea and is prone to seasonal flooding. During periods of heavy rain the river level can rise 15-20 m above basal flow (Payne, 1989).

The Lower Kinabatangan Wildlife Sanctuary (LKWS) contains the largest floodplain forest in Sabah. Habitat types are primarily described by their vegetation in this rainforest landscape. The principal vegetation type is lowland dipterocarp made up of seasonally and periodically inundated forests. These forests are unique in Sabah because vegetation has been shaped by regular flooding which influences soil salinity and composition (Azmi, 1998). According to Azmi's (1998) botanical survey, the major vegetation types within the region are freshwater swamps and swamp forests, limestone communities, mangrove forests and swamps, and lowland dipterocarp forests. The swamp forests are believed to be the largest in Malaysia (Payne, 1989).

\section{Biodiversity, deforestation \& conservation}

The island of Borneo has 221 species of terrestrial mammals, 276 reptiles, 440 freshwater fish, 633 birds, 141 frogs and 15000 plant species (Runting et al. in review). Approximately 50 of the terrestrial mammals are found in the Lower Kinabatangan region. Primates, including the Bornean orangutan (Pongo pygmaeus) and the proboscis monkey (Narsalis larvatus), are endemic as well as Borneo's three mega-herbivores: the Bornean elephant, Sumatran rhinoceros (Dicerorhinus sumatrensis) and banteng (Bos javanicus), although the latter two species have not been found in the Lower Kinabatangan region for approximately 20 years (Ancrenaz, pers. comm.). Other notable species present include the Malayan sunbear (Helarctos malayanus) and the clouded leopard (Neofelis nebulosa). The floodplains provide a wide range of habitats that harbour these diverse concentrations of wildlife, where many species are rare and endangered.

Unfortunately over the last 20 years the emphasis on developing Sabah's economy has led to significant and drastic changes in the landscape. The Kinabatangan District was one of the first areas in Sabah to be opened for logging, which reached its peak in the region in the 1970s and 1980s (Hutton et al., 2005). With the disappearance of valuable hardwood trees, 
economic policy favoured the conversion of logged forest in the Lower Kinabatangan to agriculture (Latip et al., 2013), mainly for palm oil plantations. According to Payne \& Andau (1997), the progressive disappearance and degradation of forests constitutes one of the major environmental problems in LKWS and an estimated $80 \%$ of the Lower Kinabatangan floodplain has been converted to agriculture or other human-made, non-forest land cover. As a result of these anthropogenic influences, including fires, the vegetation of LKWS is predominantly secondary growth apart from some primary forest patches located on less accessible limestone cliffs.

Before the 1980s the major conversion of forested land for agriculture was for tobacco (1890-1915), jute (1930s), rubber and coconut (1970s). Since then, oil palm has been the major crop of the lowlands where areas of forested land have been cleared for large-scale plantations (Woods, 1989; Bryan et al., 2013). Conservation efforts in Sabah intensified in the late 1970's when the State Government initiated steps to conduct biodiversity surveys in Sabah. Sites significant for wildlife conservation were identified and later declared protected areas. Initially, the Lower Kinabatangan was not among those areas identified; it was the economic policy that the forested area in the Lower Kinabatangan be converted to agriculture. However, the situation changed for the better in the 1980s when scientific research continued to produce convincing evidence of the importance of this area for wildlife conservation and as a centre of biodiversity (Hai et al., 2001).

In the late 1980s, the Sabah Ministry of Tourism and Environmental Development outlined a proposal to establish the Kinabatangan Wildlife Sanctuary. Following this, World Wildlife Fund Malaysia was commissioned by the Sabah Tourism Promotion Corporation (STPC) to conduct a study of the tourism potential of the area. The study revealed good prospects for nature tourism and also endorsed the concept of a Wildlife Sanctuary (Malim, 2002). Recognising the high value of the Kinabatangan floodplains, despite the need to modify its policy on land development, the State Government in 1992 approved in principle the need to establish conservation areas in the Lower Kinabatangan (Vaz, 1997). More recent laws require that state oil palm plantations must not extend to the riparian area within 50 metres of the river to allow movement for wildlife. However, as is the case in many developing countries, such laws are not consistently enforced. 

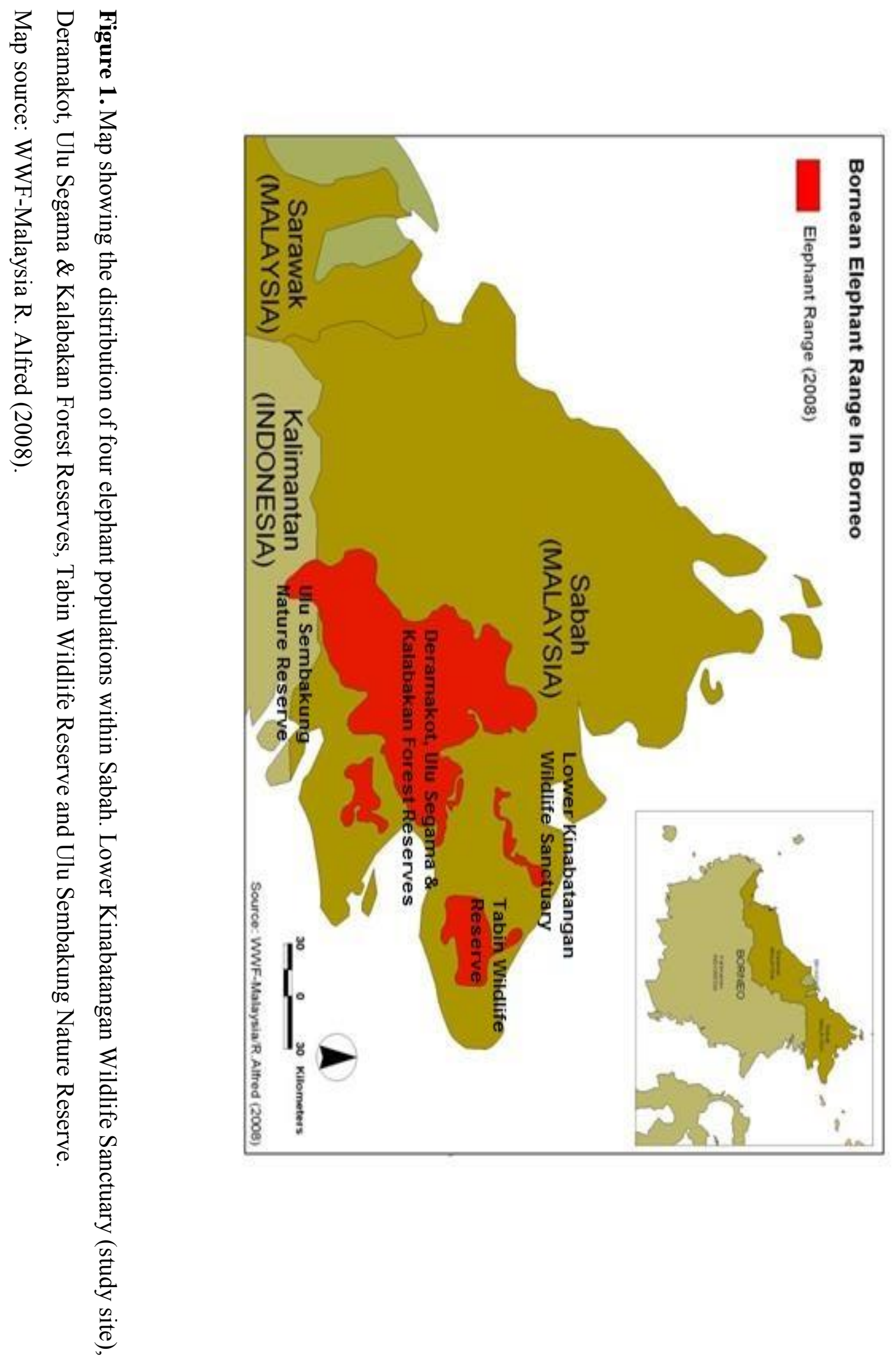


\section{Thesis structure and content}

My objective was to explore the habitat use and food-plant relationships of the Bornean elephant (Elephas maximus borneensis) by focusing on recursion both in theory and in practice. I aimed to incorporate optimal foraging theory and mega-herbivore resource-use and measure recursion at two spatial scales: site and plant. I wanted to thoroughly review the recursion literature and integrate studies exploring behaviours that resemble recursion but use different terminology. I also wanted to demonstrate how recursion can be incorporated into studies of foraging theory associated with the cost-benefit trade-off in the consumption of resources.

The main theme of this dissertation, recursion, is explored in a detailed synthesis of the topic in Chapter Two. This chapter serves as a comprehensive and quantitative literature review of the topic in the context of foraging theory. The influence of large body size on optimal foraging with respect to recursion will be presented. I will explain how the information gained from recursion studies can incorporate foraging theory and how this might be beneficial for species conservation. The recursion synthesis guides the design of the empirical chapters, including exploration of the patterns of recursion at two scales (Chapters Four and Five).

The vegetative community of the study site is not well understood, therefore before commencing my study of recursion, I embarked on a detailed description and classification of habitat types within the sanctuary and an investigation to establish the elephant population's relative preference for habitat types (Chapter Three). Habitat type classifications are discussed using quantitative analyses of floristic composition and physical characteristics. This information then informs the section on elephant habitat use:availability within the sanctuary. I hypothesised that elephant habitat use would occur in proportion to habitat availability with an avoidance of less optimal areas such as those with high human disturbance. The habitat types classified in this section inform the subsequent chapters on recursion and resource-use (Chapters Four, Five and Six).

The first empirical chapter on recursion (Chapter Four) focuses on recursion at the site level over a 12-month period. My objective was to determine what site characteristics influence recursion and how this relates to the elephants foraging strategy. I hypothesised that if recursion is part of the elephant's optimal foraging strategy then foraging site recursion will occur as a function of site quality, extent of last use, and time since last use, because these factors influence site resource status and recovery. If this is true for elephants then recursion 
sites and sites with similar qualities might usefully be incorporated into landscape management strategies for elephant conservation in the area. Recursion sites should be considered high quality for elephants, particularly if they spend more time within these sites. Chapter Four was published in a special edition of Current Zoology on Behaviour and Conservation (English et al., 2014b, Appendix Four).

Sites that receive recursion may have certain characteristics but I was also interested to test whether plant recovery rate influences recursion to foraging sites. The second empirical chapter on recursion, Chapter Five, is a study designed to explore fine-scale recursion to previously browsed plants. My objective was to understand if the elephants return to feed on plants after they have recovered. I hypothesised that if the elephants are foraging optimally then re-browsing should occur once plants have recovered. However, if re-browsing frequently occurs before plant recovery, these resources may be progressively depleted. This outcome is expected if insufficient resources are available within the study site and may be an indication that the elephant population exceeds carrying capacity of the environment. The scale of sampling required to investigate recursion to individual plants is very large because plant-recursion occurs as the final choices in a hierarchy of choices: habitat, site, and plant. This study serves to inform the design of a much larger scale study where inter-dependencies of spatial scale can be addressed. Chapter Five was published in the scientific journal PeerJ (English et al., 2015).

In addition to exploring variation in recursion due to site characteristics (Chapter Four) and then testing if recovery time contributes to explaining species and site recursion patterns (Chapter Five), I then aimed to identify elephant food-plant preferences and how plant growth characteristics influence these preferences (Chapter Six). Based on previous literature by Makhabu et al., (2006b), who explored African elephant browse preferences associated with the plant vigour hypothesis (Price, 1991), I explored whether this was true for elephant food-plant relationships for forest elephants in LKWS and whether the elephants preferred browse species that recovered faster. The Plant Vigour Hypothesis (PVH) proposes that herbivores show a preference for more vigorously growing or more vigorously recovering plants, within and between species, as these plants are considered higher quality (Price, 1991). However, larger herbivores tend to be less selective in their food choices and tend to substitute quantity for quality (Demment \& van Soest, 1985; du Toit \& Owen-Smith, 1989). I therefore hypothesised that the PVH will not apply to elephants in LKWS but that browse plant size will influence food preferences by providing access to more food with less energy expenditure than would be required to search for additional resources (Vivas et al., 
1991; Wilson \& Kerley, 2003). I also hypothesised that the elephants will prefer food plant species proportional to their availability in order to meet intake requirements. Chapter Six has been published in the Journal of Tropical Ecology (English et al., 2014c).

I conclude with a synopsis of what the thesis contributes to elephant landscape management strategies in the LKWS and our understanding of large herbivore resource relationships. Suggestions for future research into herbivore foraging and recursion are discussed. I aimed to contribute to knowledge of plant-mega-herbivore relationships and to the limited ecological literature of the Bornean elephant. I also intended to use the information gathered in this study to inform conservation professionals and wildlife practitioners of ways to optimise Bornean elephant habitat-use in existing forested areas, to restore degraded habitat, and to design and locate corridors that link forest fragments. 


\section{Chapter Two}

Recursion and its application to foraging by a mega-herbivore 


\begin{abstract}
Herbivores may use the landscape in ways that are efficient but also in ways that enhance resources. Both of these outcomes can be achieved by the repeated use of plants, plant communities or foraging sites over varying temporal scales, a behaviour known as recursion. Recursion patterns are fundamental to understanding resource-use by herbivores as they are a product of how an animal adjusts to, and influences, environmental and biological heterogeneity in space and time. Few studies have explored recursion, or behaviours similar to recursion, in uncontrolled environments and on wild animals, and even fewer have explored it in large herbivores. Studies of recursion that incorporate vegetation depletion and renewal rates can determine if herbivores are utilising resources optimally, such as returning to sites once resources have fully recovered, or returning too soon and over-utilising them. Furthermore, not only can studies of recursion improve our understanding of plant-herbivore relationships but also its influences on plant community structure. The predictions that optimal foraging theory makes for recursion, and its implications for foraging herbivores, are discussed in this review and synthesis of current knowledge about recursion. How recursion might be incorporated into studies of resource-use and the integration of a disparate literature from this review is then used to guide the design of later empirical chapters.
\end{abstract}




\section{Introduction}

Feed quality and the spatial distribution of foraging patches may influence the foraging strategies of herbivores and the way in which they utilise the landscape (Owen-Smith \& Novellie, 1982; Belovsky, 1984; Pyke, 1984; Adler et al., 2001). Herbivores may use the landscape in ways that are efficient but also in ways that facilitate improved resources. Both of these outcomes can be achieved by the repeated use of plants, plant communities or foraging sites over varying temporal and spatial scales. This repeated use is known as 'recursion'. Although this behaviour is likely to be ubiquitous and central to the theory of optimal foraging, it has been explored very little in wild animals, or in a way that incorporates foraging theory (Bar-David et al., 2009; Riotte-Lambert et al., 2013). Understanding recursion patterns is fundamental to recognising the factors that govern resource and home-range use and placement as these are a product of how an animal adjusts to environmental and biological heterogeneity in space and time (Patterson et al., 2008; BarDavid et al., 2009; Benhamou \& Riotte-Lambert, 2012; Riotte-Lambert et al., 2013). Recent studies of recursion movement have shown that it does occur in wild herbivores, and it is suggested that this type of behaviour is part of an animal's optimal foraging strategy (BarDavid et al. 2009; Wolf et al. 2009; Li et al. 2012; Benhamou \& Riotte-Lambert, 2012).

\section{Optimal foraging theory}

Optimal foraging theory argues that diet selection by generalist herbivores is significantly influenced by trade-offs between the advantage of consuming a given diet component and the cost of searching for it (Stephens \& Krebs, 1986). Travel time and distance travelled in search of food cost herbivores considerable energy (De Vries et al., 1989). Hence, foraging decisions that minimise energy expenditure are likely to be more efficient. Less efficient strategies are expected to be selected against. To minimise energy expenditure and make optimal use of its home-range, a herbivore would benefit by evaluating vegetation depletion and renewal rates (McNaughton et al., 1997). This strategy would reduce the occurrence of returning to areas exploited recently, or returning to areas with little positive feedback such as poorer quality sites.

Herbivores may also increase resource productivity and quality leading to increased future consumption, thus creating a positive consumer-resource loop (McNaughton, 1997; de Mazancourt et al., 1998; Holdo et al., 2007). Evidence of herbivory enhancing resource quality and availability has been investigated across a variety of landscapes, including 
African savanna (McNaughton, 1988), North American prairies (Detling \& Painter, 1983), European temperate grassland (Bos et al., 2005), and arctic tundra (Olofsson et al., 2001). Increased leaf productivity (McNaughton, 1976; Cargill \& Jefferies, 1984; Ruess \& McNaughton, 1984; Bazely \& Jefferies, 1989; Hik \& Jefferies, 1990) and an increase in leaf nutrient concentration (McNaughton, 1979; McNaughton et al., 1997; Coppock et al., 1983; Ruess et al., 1984; Olofsson et al., 2004) has been related to herbivory (Cromsigt \& Kuijper, 2011). Moreover these returns may accelerate nutrient cycling in highly grazed sites (McNaughton et al., 1997). Nevertheless, these relationships depend on the plant growth potential as some plants are non-renewing or very slow to renew, causing patches to be progressively depleted (Owen-Smith, 2002). Herbivores may therefore utilise resources in such a way as to encourage long-term optimisation of return, rather than by exploiting and depleting them in the short-term (Gordon \& Lindsay, 1990). For example, recursions may be part of an optimal foraging strategy for herbivores timing returns to feed on recovering resources in a high stage of productivity. Conversely, some plants produce more chemical and physical deterrents as a response to herbivory, such as toxins or spines, which presumably add a cost to the herbivore (Karban \& Myers, 1989).

Optimal foraging strategies focus on patch choice, patch-use and patch departure, but Charnov's (1976) model is limited in two ways because it assumes that food is obtained by foragers continuously and that a forager automatically knows, at any point in time, the exact rate at which food is being consumed (Oaten, 1977). Optimal foraging has heuristic value (Pyke, 1984) but it is recognised that animals rarely behave exactly optimally. Less than 'optimal' foraging can result from environmental unpredictability, incomplete knowledge, or interactions between the two (Dall et al., 2005). The marginal value theorem (MVT) (Charnov, 1976) suggests that a herbivore should abandon a resource patch when forage is depleted to the local average. Animals might therefore avoid previously visited sites (i.e. negative correlation between spatial familiarity and site selection) when resources are depleted below the local average (Wolf et al., 2009).

Two commonly referred to theories associated with a cost-benefit trade-off in the consumption of a resource are giving up densities (GUD) and ideal free distribution (IFD) (Fretwell \& Lucas, 1970; Charnov, 1976). The theory of GUD predicts that patches of resources, or sites, are utilised until the cost of utilising them further is greater than the cost of moving to the next patch, or the potential gain of utilising them further is less than the gain from searching for another patch (Charnov, 1976). If GUD is a true representation of how large herbivores use habitat, then recursion to a site should not occur by animals from the 
same group after using it, until the site has recovered. Alternatively, if they do return to the site before recovery, they should not have exhausted the resources at the previous visit.

IFD predicts that animal density or utilisation per unit resource should be the same across the landscape because the higher the quality of the habitat the larger the density of animals it can support. Although higher quality sites have more resources, they support more animals of the same species. Lower quality sites support fewer animals (Fretwell \& Lucas, 1970). If IFD is a true representation of how animals use habitat then the utilisation of a site and how frequently recursion occurs should happen in proportion to the site's relative quality.

Patterns of resource-use may also be facilitated by individual ability to learn and recall the location of previously profitable areas. For example, inherited attributes and experience, individual and social learning systems, predispositions toward novel stimuli, and spatial memory are likely to contribute to foraging decisions (Fryxell, 1995; Adler et al., 2001; Redfern et al., 2003; Launchbaugh \& Howery, 2005). According to Wolf et al. (2009), if spatial familiarity does not determine site selection then there would be no evidence of preferential selection of previously visited locations. This would be the case if there is little difference in quality between recursion and non-recursion sites. Moreover, an animal may return to a site for the same reason it selected it in the first place - high resource availability. However, if spatial memory does influence site selection we would expect animals to return to higher quality sites and avoid lower quality sites for improved foraging success (Wolf et al., 2009). 


\section{Previous studies of recursion}

Bar-David et al. (2009) introduced the term 'recursion' as a movement pattern in wild buffalo (Syncerus caffer). They provided a qualitative indication of the time-scale at which recursions occurred but did not outline specific areas of high-use. Li et al. (2012) went further by proposing to directly apply the method used by Bar-David et al. (2009) to a time series of presence/absence events in a given area to detect periodicity in recursions using a simulation model. Despite their approach focusing on highly used areas this technique also did not identify favourable foraging areas (Riotte-Lambert et al., 2013). For instance, some areas, such as track crossings, can be visited often without involving high residence times and therefore areas with preferred resources are likely to be underestimated using this approach (Riotte-Lambert et al., 2013). The third and most recent study of recursion by Riotte-Lambert et al. (2013) on impala (Aepyceros melampus) identified areas of particular interest in terms of mean residence time per visit and recursion frequency.

These former works of identifying and assessing recursion movement show the frequency of recursion, the time period over which recursion occurs and, in the latest paper by Riotte- Lambert et al. (2013), its relationship with the intensity of site use. They do not, however, answer questions about what influences these recursion patterns, such as its relationship with site characteristics, the relationships between residence time and the time between recursions, and whether these metrics and relationships are supported by foraging theory. An empirical and systematic study of these influences within a theoretical framework is yet to occur.

Recursion is a relatively new terminology in the ecological literature. But studies pertaining to processes that lead to recursion or that are consequences of recursion have been explored extensively, particularly in controlled environments. I explore the historical trend in the contribution of recursion and related studies (specifically spatial memory and site reuse, site-fidelity and optimal foraging theory). I then quantify the amount of literature on animal site reuse behaviours to illustrate the scarcity of studies available for large herbivores compared to other smaller vertebrates (Figure 1 and Appendix Six). 


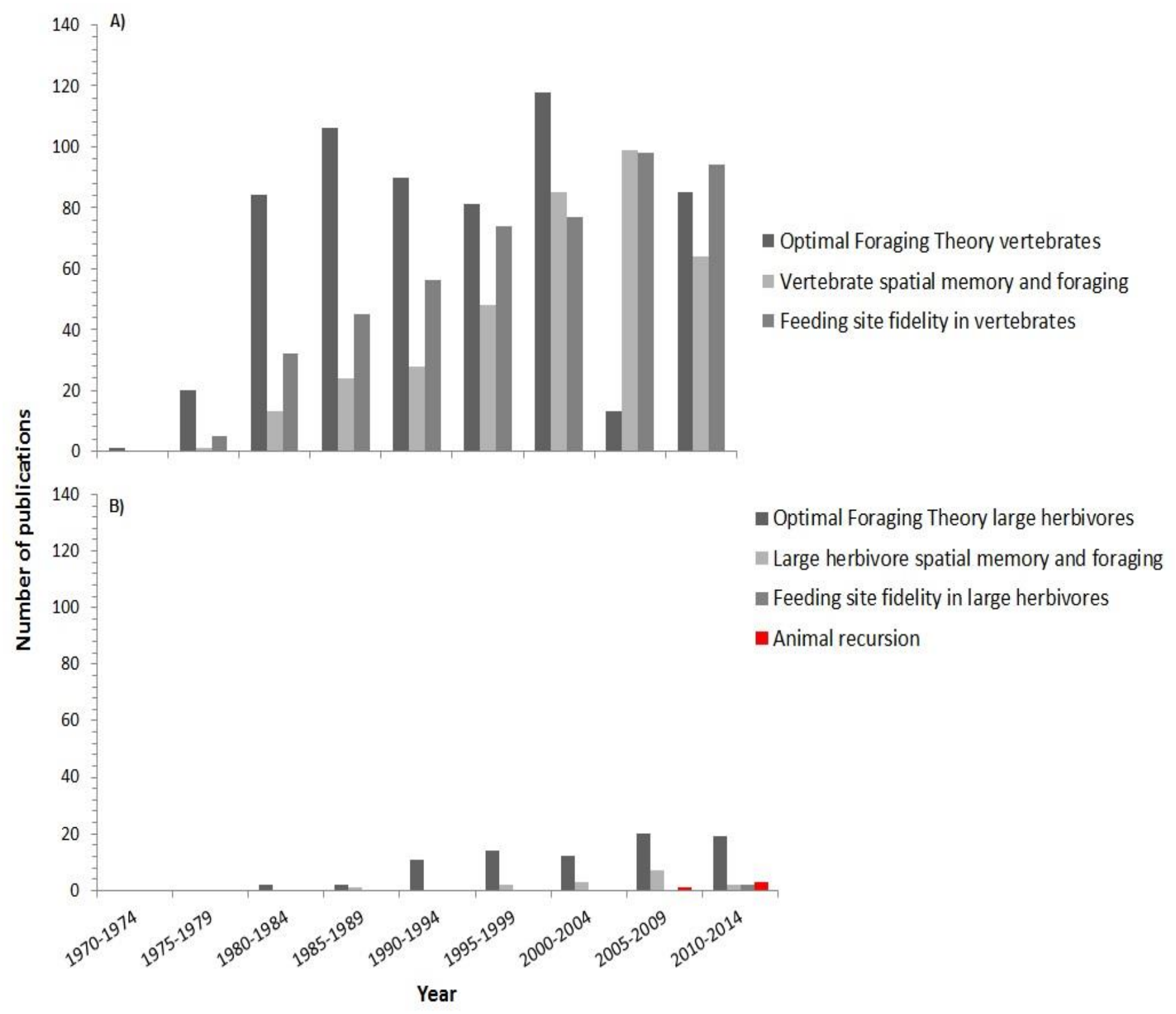

\section{Figure 1.}

Literature associated with A) vertebrate foraging studies (not including large herbivores) compared to the literature available related to B) large herbivore foraging was quantified between the years 1970 - 2014 using a scientific database (Web of Science). Literature associated with the following terms were quantified: 'Optimal foraging theory vertebrates', 'vertebrate spatial memory and foraging', 'feeding site fidelity vertebrates', optimal foraging theory large herbivores', 'large herbivore spatial memory and foraging', 'feeding site fidelity large herbivores' and 'herbivore recursion'. Publications included in the figure are located in an electronic file (Appendix Six) and are listed in order of publication date for each of the above topics. 
Spatial memory of resource locations may greatly improve foraging success. Temporal patterns in site revisits have been related to resource recovery (e.g. in insects (Bell, 1990; Williams \& Thomson, 1998) and birds (Motacilla sp.) (Davies \& Houston, 1981)). The predictability and order of repeated visits to previously utilised sites has been explored extensively in bumblebees (Bombus sp.) (Comba, 1999; Heinrich, 1976; Manning, 1956; Thomson, 1996; Thomson et al., 1982, 1987), euglossine bees (Euglossini spp.) (Janzen, 1971; Ackerman et al., 1982; Dressler, 1982), butterflies (Danaus sp.) (Gilbert, 1980), hummingbirds (Phaethornis spp.) (Gill, 1988; Garrison \& Gass, 1999; Tiebout, 1991), wagtails (Motacilla sp.) (Davies \& Houston, 1981), bats (Glossophaga sp., Pippistrellus sp.) (Lemke, 1984; Racey \& Swift, 1985), and primates (Saguinus spp., Cebus sp., Gorilla sp.) (Garber, 1988; Janson, 1998; Watts, 1998) - species for which foods are highly heterogeneously distributed because they are flowers and fruits. Additionally, the influence of resource renewal on site reuse has been explored using simulations of animal movement patterns (Possingham, 1989; Bar-David et al., 2009; Ohashi \& Thomson, 2005). Nevertheless, only a few studies have explored foraging site reuse in uncontrolled environments and these studies are largely limited to primates (Garber, 1988; Watts, 1998; Garber \& Jelinek, 2006; Erhart \& Overdorff, 2008; Porter \& Garber, 2013; Janmaat et al., 2006) and a few ungulates (Syncerus sp., Aepyceros sp.) (Bar-David et al., 2009, RiotteLambert et al., 2013) (Table 1). The concepts are not well described or tested, but especially undeveloped for large wild vertebrates in uncontrolled habitats.

Site-fidelity, which can be a consequence of recursion, is the tendency of an animal to return to a previously occupied area or remain within the same area for an extended period of time (White \& Garrott, 1990). This behaviour has been documented in a variety of species including birds and mammals (Greenwood \& Harvey, 1982; Switzer, 1993; Wittmer et al., 2006) and is often a component of breeding behaviour (Schmidt et al., 2007), the result of social interactions (Wolf \& Trillmich, 2007), or the outcome of an animal being territorial (Switzer, 1993; Börger et al., 2008). Site fidelity may also be due to the efficient exploitation of resources in areas that are adequate for food, mates and protection (Switzer, 1997; Barraquand \& Benhamou, 2008) and can subsequently improve our understanding of the underlying mechanisms that result in an animal's use of its seasonal range (Schoen \& Kirchhoff, 1985; Aycrigg \& Porter, 1997). Recursion to foraging sites, in conjunction with breeding, territoriality and social interactions, may therefore lead to site-fidelity over time. 
Table 1. Current literature on recursion and foraging site reuse by vertebrates in uncontrolled environments where bold represents studies that use the terminology 'recursion'. Studies of recursion amongst free-ranging vertebrates have, so far, been limited to studies of primates and ungulates.

\begin{tabular}{|c|c|c|c|}
\hline Authors & Year & Location & Species \\
\hline Garber & 1988 & $\begin{array}{l}\text { Amazon basin, } \\
\text { Northeastern Peru }\end{array}$ & $\begin{array}{l}\text { Moustached (Saguines } \\
\text { mystax) \& Saddle-back } \\
\text { (Saguinus fuscicollis) } \\
\text { Tamarins }\end{array}$ \\
\hline Watts & 1998 & $\begin{array}{l}\text { Karisoke Reasearch } \\
\text { Centre, Rwanda }\end{array}$ & $\begin{array}{l}\text { Mountain Gorilla } \\
\text { (Gorilla gorilla } \\
\text { beringei) }\end{array}$ \\
\hline Garber \& Jelinik & 2006 & $\begin{array}{l}\text { Isla de Ometepe, } \\
\text { Nicaragua }\end{array}$ & $\begin{array}{l}\text { Nicaraguan Mantled } \\
\text { Howler Monkey } \\
\text { (Alouatta palliata) }\end{array}$ \\
\hline Erhart \& Overdorff & 2008 & Madagascar & $\begin{array}{l}\text { Lemur (Propithecus } \\
\text { edwardsi \& Eulemur } \\
\text { fulvus) }\end{array}$ \\
\hline Wolf et al. & 2009 & $\begin{array}{l}\text { Rocky Mountains, } \\
\text { Canada }\end{array}$ & Wapiti (Cervus elaphus) \\
\hline Bar-David et al. & 2009 & $\begin{array}{l}\text { Klaserie Private } \\
\text { Nature Reserve \& } \\
\text { Kruger National Park, } \\
\text { South Africa }\end{array}$ & $\begin{array}{l}\text { African Buffalo } \\
\text { (Syncerus caffer) }\end{array}$ \\
\hline $\begin{array}{l}\text { Benhamou \& Riotte- } \\
\text { Lambert }\end{array}$ & 2012 & $\begin{array}{l}\text { W Regional Park, Niger } \\
\text { Basin }\end{array}$ & $\begin{array}{l}\text { African Buffalo } \\
\text { (Syncerus caffer) }\end{array}$ \\
\hline $\begin{array}{l}\text { Riotte-Lambert et } \\
\text { al. }\end{array}$ & 2013 & $\begin{array}{l}\text { Hwange National Park, } \\
\text { Zimbabwe }\end{array}$ & $\begin{array}{l}\text { Impala (Aepyceros } \\
\text { malampus) }\end{array}$ \\
\hline Porter \& Garber & 2013 & North Bolivia & $\begin{array}{l}\text { Weddell's Saddleback } \\
\text { Tamarin (Saguinus } \\
\text { fuscicollis weddelli) }\end{array}$ \\
\hline Janmaat et al. & 2013 & $\begin{array}{l}\text { Tai National Park, Cote } \\
\text { d'Ivoire }\end{array}$ & $\begin{array}{l}\text { Chimpanzee (Pan } \\
\text { troglodytes verus) }\end{array}$ \\
\hline
\end{tabular}




\section{Recursion at two scales: site and plant}

A foraging site is a collection of patches in a contiguous spatial area that animals graze during a foraging bout (Bailey et al., 1996). Recursion rates and the time an animal allocates to foraging sites per visit can indicate their attractiveness and value to a population or species, particularly when compared to sites that do not receive recursion. The unique characters of sites at which foraging recursion occurs, such as floristic composition and biophysical features can be used to identify the biotic and abiotic drivers of site recursion. Foraging would be more efficient if the interval between visits (recursions) to sites reflected the extent of previous use and the profitability of the site. For example, animals might allow more time to pass before returning to a site if resources were previously exhausted or they might return sooner if resources within a site were utilised sparingly.

At the plant level, if herbivores monitor vegetation renewal rates and re-browse on plants after they have recovered, this would also increase their foraging efficiency. If this were the case then sites with slowly recovering plants may show less frequent recursions than those sites with faster recovering species. If a herbivore returns to a site and feeds frequently on unrecovered plants, resources would be progressively depleted and this may lead to the carrying capacity of the environment being reduced. Recursion to individual plants, and sites, is therefore expected to be strongly determined by plant recovery after herbivory.

\section{Recursion by elephants}

\section{Body size}

Body size is a key factor influencing the foraging decisions of mammalian herbivores. Due to lower mass-specific metabolic requirements and a larger gut volume, large herbivores are better able to survive on lower quality food compared to small herbivores (Bell, 1971; Jarman 1974; Demment \& van Soest, 1985). Large herbivores tend to trade quality for quantity, as the benefits of ingesting abundant forage resources outweigh the costs of searching for forage of higher nutritional return, which is usually rarer in the environment (Demment \& van Soest, 1985; Owen-Smith, 1988). Elephants are the largest land mammal, requiring large quantities of resources to meet their nutritional and metabolic needs (Benedict, 1936). A study of recursion and foraging behaviour by elephants therefore represents the uppermost extreme of 
the relationship between a herbivore and its reuse of sites or resources. Reuse of profitable areas, or recursion, may be a foraging strategy employed by elephants to maximise their feed intake and minimise energy expenditure. We might also expect a positive feedback between repeated use and resource quality. However, there are reasons why we might also expect elephants to be less recursive than other herbivores. For example, we would expect large herbivores like elephants to return to foraging sites only when food plants have sufficiently replenished because they are bulk feeders. Elephants might also be less recursive than smaller herbivores, due to larger herbivores having a reduced requirement for high quality food (i.e. food plants do not need to be at the optimal stage of regrowth). Furthermore, more resources would be consumed per visit resulting in more time for resources to replenish and therefore less frequent recursions. On the other hand, elephants have a highly developed spatiotemporal memory (Hart et al., 2008). I would expect the capacity of elephant, therefore, for well-developed, carefully timed spatio-temporal reuse of resources to be greater.

\section{Influence of spatial memory and social learning}

Anecdotally, elephants have long been considered to have a relatively high capacity for memory, for example, retention of spatial and temporal knowledge - and this idea has been supported in empirical studies conducted on elephant cognition (Bates et al., 2008; Hart et al., 2008). Hart et al. (2008) found that elephants appeared to excel in long-term, extensive spatial-temporal and social memory. According to Fagan et al. (2013) spatial memory provides animals with many advantages. At local scales, these benefits include improved choice of critical locations, such as food patches. At larger scales, spatial memory aids navigation in landscapes, and relocating essential sites or resources that are only available periodically (Bingman \& Cheng, 2005; Janmaat et al., 2006; Papastamatiou et al., 2013). Long-term memory should be especially valuable in landscapes with a predictable spatial and temporal distribution of resources, where remembering locations and their attributes would be beneficial in increasing fitness through more directed movements or timely returns (Fagan et $a l ., 2013)$, such as recursions. On the other hand, memory would also be beneficial in changing or unpredictable environments where associative learning allows animals to adapt to recent changes in their environment as opposed to innate foraging behaviour.

To forage optimally, elephants may use their spatial memory to return to areas within their home-range where, in the past, they have gained positive nutritional feedback. This process might enable more time for ingesting replenished or higher quality or quantity (larger 
individual or large numbers of) food plants, thus increasing foraging efficiency, rather than moving through the landscape and sampling food randomly. One must bear in mind, however, that the existence of recursions is not always a reliable indicator of memory, particularly at the fine-scale (Benhamou \& Riotte-Lambert, 2012). At a fine-scale, recursion may stem from the remote perception and response to a stimulus indicative of a favourable site. At a large-scale, it is more likely that recursions are governed by spatial memory because revisited areas are beyond an animal's immediate perceptual range (Benhamou \& Riotte-Lambert, 2012).

Elephants may maintain spatial and temporal knowledge of food distribution, quantity and quality, but they may also utilise areas as a way of 'information gathering' (Bailey et al., 1996). If this is so, then one might predict that elephants may use a newly discovered or resampled site lightly but return to it later to fully exploit it after knowing its high value relative to other known sites; that is, recursion is associated with site-sampling. In this case site acceptance may be manifested over lengthening time-scales (Owen-Smith, 2002). If this is how elephants forage, recursion should also occur over short time-scales, by the same groups, and the second and subsequent visits would involve greater utilisation than the first. This would be evidence for optimal habitat-use by GUD under the constraint of imperfect knowledge. One would also expect the time to recursion and amount of utilisation to correlate with site quality (IFD). For example, the higher the quality of the site the shorter should be the time to recursion and residence time greater. However, if recursion occurs over longer time scales and time between returns corresponds to food-species recovery then it is evidence for the operation of GUD with perfect information. The quality of the site will be indicated by the positive relationship between rate of recovery and recursion rate. In these ways current optimal foraging theory makes mutually exclusive and testable predictions about the function and pattern of recursion in elephant.

Social learning is often seen as an adaptive mechanism which allows animals to transfer the location of desirable foods and habitats, but it can also be maladaptive. Sheep (Gluesing \& Balph, 1980; El Aich \& Rittenhouse, 1988; Provenza et al., 1996), cattle (Hodder \& Low, 1978), goats (Biquand \& Biquand-Guyot, 1992), deer (Gillingham \& Bunnell, 1989), and moose (Anderson, 1991) can walk long distances searching for previously used preferred plants or sites but pass areas with abundant forage along the way. Geist (1971) proposed that bighorn sheep (Ovis canadensis) could not expand their established home ranges because young animals conformed to the habitat-use patterns of their 
predecessors. Thus, social learning adds another dimension to investigations of recursion, especially for social, long-lived animals like elephant.

Given the social cohesiveness of elephant herds and the strength of social bonds among elephants, there seems to be ample opportunity for social learning to occur (Greco \& Brown, 2013). All species of elephants form large social networks with hierarchical, multilevel organisation, which suggests that elephants have a high level of social complexity (Bates et al., 2008). Weaning occurs at 4 years but daughters remain with their mother for life, resulting in a semi-permanent group of related females. A matriarch, usually the oldest female, leads each family (Moss, 2012).

In elephants, patterns of recursion to sub-optimal areas may result from information passed on from the matriarch of the herd. Tight cohesion among familiar herd members could, therefore, constrict habitat-use patterns and keep individual animals from seeking or exploring new and perhaps more optimal foraging sites and result in recursion 'errors' - the return to previously used sites that are not profitable. For instance, brief, foraging-poor visits to revisited sites, or neglect of other higher quality but nonetheless also available sites, might indicate recursion errors. If social learning-induced recursion errors occur in elephants then we would expect no difference in the quality, time spent at sites and time period between returns for recursion and non-recursion sites.

\section{Technological advances facilitate studies of recursion}

The literature of large herbivore site reuse, which is currently far less than for smaller vertebrates (Figure 1 and Table 1) is expected to increase with developments in animaltracking technology. Before GPS technology, a study of recursion by wild animals in an uncontrolled environment would be labour intensive and logistically challenging. GPS technology now enables precise and inexpensive ways of monitoring and mapping animal movement - securing vast quantities of data over varying temporal scales. Animal positions provide the elemental unit of movement paths and show where individuals interact with the ecosystems around them (Cagnacci et al., 2010). Incorporating on-ground ecological measurements and direct observations whilst quantifying recursion patterns using GPS technology can provide a more robust assessment of what influences animal resource-use over a variety of spatial scales. Future studies of recursion should incorporate all of these research techniques to address recursion frequency, residency time, time between returns and 
site composition including floristic composition and physical characteristics. This information can identify the location of critical sites and what qualities influence this value at a species-specific level.

\section{Recursion, ecology and conservation}

I have described how a study of recursion can test foraging theory in large herbivores. Foraging efficiency is expected to increase if the period between returns to sites to feed reflects how extensively the site was previously utilised. Site quality is also expected to influence recursion where higher quality sites should receive more recursions, similarly to the expectations of the Ideal Free Distribution but through time rather than in space. To improve the cost:benefit trade-off in consuming resources within foraging sites herbivores would also benefit by returning to sites after resources have recovered. These expectations highlight the importance of exploring recursion at different spatial scales such as at the site and plant level.

My review of the literature has demonstrated the need for integrating the large amount of research on spatial memory and site reuse, resource recovery and site reuse, and foraging site-fidelity with the new research topic of recursion. Furthermore, I quantified the scarcity of studies of recursion and recursion processes, like spatial memory and resource recovery, in large, wild herbivores. Studies of recursion would benefit from a better understanding of spatio-temporal memory and social learning, but studies of recursion are also an opportunity to investigate the role of spatio-temporal memory and learning in ecology of free-ranging animals.

Understanding recursion ecology can inform conservation planners of animal resource-use needs and identify habitat areas and resources within these areas that are of high conservation priority. The wider implications of recursion can lead to an understanding of the formation of animal home-ranges and migration routes, informed by the distribution and location of critical resources that are identified by studies of recursion. Wildlife practitioners and managers may then incorporate this information into the design of reserves and the location or placement of corridors linking these profitable areas to ensure ongoing access. Furthermore, patterns of herbivore recursion will contribute to understanding animal foodplant relationships and the influence of this behaviour on plant-community dynamics. For example, if herbivores are facilitating and engineering growth of certain species (Olff \& Ritchie, 1998). 
The material reviewed in this chapter guided my design of Chapters Four and Five: site and plant level recursion by the Bornean elephant and how patterns of recursion at these levels might be used to advise policy makers of landscape management strategies that benefit elephant conservation in Sabah. The main threat to the endangered Asian elephant (Elephas maximus) is habitat loss caused by human encroachment (IUCN Redlist, 2014). This is also true for the Asian sub-species, Elephas maximus borneensis. The habitat in which this subspecies is found is highly fragmented and in most cases the elephants are restricted to forest fragments surrounded by oil palm plantations (Elaeis guineensis). Understanding the relationship between this mega-herbivore and its remaining resources is crucial to their conservation and management of their remaining habitat. 


\section{Chapter Three}

Habitat classification, availability and use by elephants in the Lower Kinabatangan Wildlife Sanctuary, Sabah. 


\begin{abstract}
The habitat preferences of elephants in the Lower Kinabatangan Wildlife Sanctuary were explored in relation to habitat availability. Habitat preferences are assumed to reflect their ecological importance to wildlife and it is therefore crucial to identify them in order to design effective management strategies. I expected elephants, as generalist herbivores, to utilise a wide range of habitat types proportional to their availability but avoid those with poor resources or high risk. The floristic composition and physical characteristics of all habitat types within the elephant population range had not been previously quantified. I conducted a habitat type classification analysis and identified two additional habitat types not previously described. Variation between four habitat types was identified using multidimensional scaling. Ten randomly placed strip-transects, $1 \mathrm{~km}$ long and $4 \mathrm{~m}$ wide, were used to quantify the availability and use of habitat types by elephants. All elephant dung piles, footprints and feed-sign were recorded within strip-transects as an index of habitat use. Elephant dung and footprints could not be used in the analysis because forest-floor flooding in two habitat types, swamp and semi-inundated forests, made them less detectable. Thus, only feed sign was used. I found that the elephants were selective of one habitat type that had not previously described for this study site, open grass areas along forest margins, despite this habitat type being one of the least common (used 32\%, available 14\%). The elephants avoided swamps (used $6 \%$, available 21\%) and recently logged or cultivated areas (used 6\%, available 11\%) and selected lowland mixed dipterocarp and semi-inundated forest in proportion to their availability (used $34 \%$, available $34 \%$ and used $21 \%$, available $22 \%$ respectively). This result highlights the importance of open grass areas for the elephant population and therefore the need for this habitat type to be protected and incorporated into landscape management strategies in the Lower Kinabatangan.
\end{abstract}




\section{Introduction}

Central to the study of animal ecology is the use an animal makes of its environment, specifically the kinds of foods it consumes and the varieties of habitats it occupies (Johnson, 1980). Foods and habitats are resources to animals. A preference for a resource is described when it is shown to be used substantially more than it is available. Resources used disproportionately more than they are available are also said to be 'selected' and those used less 'avoided' (Manly et al. 2002). Animals' preferences, and selection or avoidance of resources, are defined, therefore, by their departure from random use (Johnson, 1980) and are relative metrics whose values are conditional on the availability of all other resources (foods or habitats) to the animal (Aarts et al., 2008).

Resource selection and avoidance may take place at different spatial scales, the largest of which is at the scale of habitats within the range of a population (Johnson, 1980). Measuring animal habitat selection and avoidance is essential because it allows for the identification of habitats that are influential in the distribution and abundance of wildlife populations because those habitats contain critical resources or because they do not (Beyer et al., 2010). Habitat preferences, therefore, are assumed to reflect their ecological importance to wildlife populations and measuring them is a common beginning for many ecological studies of wildlife (Manly et al., 2002).

Elephant habitat selection and avoidance (use:availability) has been extensively researched for Asian (Elephas maximus) and two African species (Loxodonta africana and Loxodonta cyclotis). Studies have informed us of elephant preferences and seasonal and diurnal influences on these preferences (Sukumar, 1989; Steinheim et al., 2005) such as the influence of seasonal water availability and forage quality (Laws, 1970; Sukumar, 1989; De Boer et al., 2005; Steinheim et al., 2005; Galanti et al., 2006; Shannon et al., 2006; Kumar et al., 2010). The influence of body size and social behaviour has also been explored to explain differences in habitat use between male and female elephants (Stokke \& du Toi, 2002; Shannon et al., 2006). Patterns of habitat use have been used to understand elephant movement at different spatial scales, such as at the habitat or site scale (De Knegt et al., 2011; Shrader et al., 2012) and in the development and structure of home-ranges (De Boer et al., 2005; Wall et al., 2011; Sitompul et al., 2013). 
Habitat-use patterns have informed conservation practitioners of crucial elephant habitat requiring protection as well as ways in which to reduce human-elephant conflict by describing the predictability of elephant movements across the landscape (Galanti et al., 2006; Harris et al., 2008). Habitat use studies where elephants encounter forest logging, other causes of deforestation, and conflict with humans (Rood et al., 2010; Estes et al., 2012) have found that elephants avoid areas with human presence (Theuerkauf et al., 2001; Harris et al., 2008; Kumar et al., 2010) and prefer areas with virgin or re-established forest vegetation and riparian habitats (Kumar, 2010).

Habitat selection and avoidance has not been investigated in the Bornean elephant (Elephas maximus borneensis), but studies on their movement through fragmented and nonfragmented forest have found that use of habitat ordinarily expected to be preferred is impacted by human encroachment (Alfred et al., 2012; Estes et al., 2012). A habitat use study has been somewhat prevented by the absence of quantitative assessments of habitat types floristic composition and physical characteristics - within the elephant population's range in the Lower Kinabatangan Wildlife Sanctuary (LKWS). Some habitat types were described semi-quantitatively by Azmi (1998) but these have not been published and need to be evaluated empirically. Azmi (1998) classified 6 habitat types: lowland mixed and hill dipterocarp forest, limestone forests, seasonal swamps, tidal swamp forest, permanent swamp and mangroves. However, the methods for establishing the classifications are unclear and two of these habitat types are not within the elephant's range (tidal swamp forests and mangroves).

My objective for this chapter was to categorise quantitatively the habitat types available to elephants within the LKWS and measure their use at the broad spatial scale, across their range, during the mid to late dry season in order to identify habitat types of ecological importance to this population. First, I conducted a multidimensional scaling analysis to identify the different habitat types available to elephants. I use the outcome of this analysis to offer a quantitative modification on Azmi's scheme. Using estimates of those habitat types for use and availability, I then quantified elephant selection and avoidance (or neutrality) of those habitats by the elephant population. Elephants, as large generalist herbivores, possess a wider food quality tolerance than smaller herbivores and so a more diverse and even use of habitat was expected (Owen-Smith, 1988) where utilisation of a variety of habitat types should occur proportional to their availability. However, the LKWS is 
also a highly human-impacted landscape and so I also expected elephant habitat use to be influenced by human use of the landscape and incursions into the sanctuary.

\section{Method}

This study was conducted in the mid to late dry season (June - August 2010) when flooding is less likely to limit access to elephant habitat and travel along sampling transects, especially in the riparian zone and forest swamps.

Measurements of environmental and biological variables were taken along 10 randomly distributed $1 \mathrm{~km}$ transects positioned perpendicular to the Kinabatangan River within the LKWS and the area used by elephant. A distance of $1 \mathrm{~km}$ was selected because beyond this distance the landscape is predominantly oil palm plantations surrounded by trenches and/or electric fences and is not part of the sanctuary. Locations of transects were determined by placing a grid over a map of the sanctuary; each square in the section of the grid aligned with the river edge was numbered 1 to 80 and 10 were randomly drawn to determine the approximate start point of each $1 \mathrm{~km}$ transect. If the randomly selected location was too narrow (i.e. $<1 \mathrm{~km}$ ) a new location was selected (See Figure 1, Chapter 4, page 78 for transect locations).

\section{a) Habitat classification}

Floristic composition along transects was measured every $100 \mathrm{~m}$ and was recorded by presence/absence of plant taxa within a $5 \mathrm{~m}$ radius of each point. A total of 41 plant species were recorded. Plant families not represented by at least 10 individuals across all transects were not included in the dataset for analysis. Plant families removed from the analysis due to small sample size were Pterydophyta, Phyllanathaceae, Dipterocarpaceae, Rubiaceae, Annonaceae, Moraceae, Anacardaceae, Sapindaceae, Melastomaceae, Guttiferae, Sterculiaceae and Vitaceae. Plant families included in the analysis were Leguminosae, Arecaceae, Euphorbiaceae, Myrtaceae, Dillineaceae, Poaceae, Cyperaceae, Lauraceae, Ebenaceae and Zingiberaceae.

Environmental variables were also measured at the same sites every $100 \mathrm{~m}$ and included elevation $(\mathrm{m})$, distance to water $(\mathrm{m})$, shade percentage and understorey cover percentage, and used in analyses with floristic composition to categorise habitat types. Elevation and distance to the nearest fresh water source (river, tributary or lake) were 
measured using a Garmin 60csx. Shade percentage was measured using a spherical densiometer. This tool enabled calculation of the percentage of the sky that was obscured by canopy. Canopy cover was reflected onto a 24 cell grid convex mirror (see Lemmon, 1956). Understorey cover was measured using a $2 \mathrm{~m}$ long PVC pipe marked every $2 \mathrm{~cm}$ along its length. The number of $2 \mathrm{~cm}$ marks covered by vegetation was counted from a distance of $5 \mathrm{~m}$ when the pipe was held horizontally $30 \mathrm{~cm}$ from the ground by an assistant. This was then converted to a percentage of understorey cover.

To identify variation in floristic assemblage and environments responsible for habitat types, I used PERMANOVA implemented in PRIMER (Clarke \& Gorley 2006). Multidimensional scaling (MDS) (Clarke \& Warwick, 1994) used a priori habitat type classifications from Azmi's (1998) study as well as my own habitat type additions of semiinundated dipterocarp forest and open grass areas. Recently cleared or cultivated areas were not included in the analyses because these areas were relatively void of plants (except for oil palm) and contained man-made structures. The Bray-Curtis index was chosen for environmental variables and plant composition (Beals, 1984) and the Jaccard Index was selected due to presence/absence data being used (Real \& Vargas, 1996). The environmental variables and floristic composition responsible for dissimilarities between habitat types were identified using Similarity Percentage analysis (SIMPER).

\section{b) Habitat availability}

Habitat type, based on my habitat classifications from the analysis in (a), was recorded retrospectively at $100 \mathrm{~m}$ intervals along each of the $101 \mathrm{~km}$ randomly distributed transects. The number of recordings within each habitat type was compared to the total number of records to estimate the proportionate availability of each habitat type. Three habitat types in the Lower Kinabatangan were not sampled by this technique; the mangrove swamps, tidal swamp forests and limestone cliffs, but these were also not used at all by the elephants and so were not represented in this study.

\section{c) Elephant habitat use}

Elephant habitat use was measured continuously along each of the 10 transects. Elephant dung, footprints and feed sign were recorded wherever they were observed within $2 \mathrm{~m}$ either side of the transect as evidence of elephant habitat use. As elephants are the largest animal in the study site their dung is comparatively larger than other animals such as wild boar (Sus 
scrofa) and sambar deer (Rusa unicolor) and therefore easy to identify (Figure 1A). Elephant feed sign differed from other herbivore feed sign as elephants tend to select larger portions of plants, often breaking branches or causing more visible disturbance in order to access young leaves or vines (Figure 1B). They are also capable of reaching forage up to $5 \mathrm{~m}$ from the ground far beyond the reach of wild boar and sambar deer. Felling trees is also common by elephants in order to access young shoots, roots and leaves. Other sign such as footprints (Figure 1C) and/or dung are often found close to feed sign. At every location of elephant sign the habitat characteristics and habitat type, based on my own and Azmi's (1998) classifications, were recorded. These habitat types were later confirmed based on the results of the habitat classification analyses in (a). I chose a $4 \mathrm{~m}$ wide strip-transect for recording all elephant sign because I could not be confident of detecting all sign at greater distances from the transect in dense forest.

I hypothesised that the permanent presence of above-ground water in swamps and semi-inundated dipterocarp forest would reduce dung and footprint visibility and, therefore, add a negative detection bias to my records of elephant sign and use in those habitat types. Nevertheless, I also suspected that the three types of elephant sign would be strongly spatially associated with each other in all habitat types. I compared the rates of the three signs across habitat types to check for detection bias. I aimed to determine, therefore, whether elephant feed sign, being more visible across all habitat types, was a satisfactory index of elephant habitat use without recourse to elephant dung and footprints. In order to do this I measured the spatial autocorrelation of these elephant use signs. The distances (metres) from dung to the nearest feed sign $(n=35)$ and from footprints to the nearest feed sign $(n=39)$ were calculated and then compared to a random selection of distances between nearest neighbour feed signs.

The frequency of counts for use of each habitat type for feeding (feed sign, $n=47$ ) and habitat availability $(n=100)$ was converted to a percentage of total use or availability for presentation in the figures. A chi-square test was used to determine if there was any significant difference between habitat use for feeding and availability for each habitat type. 

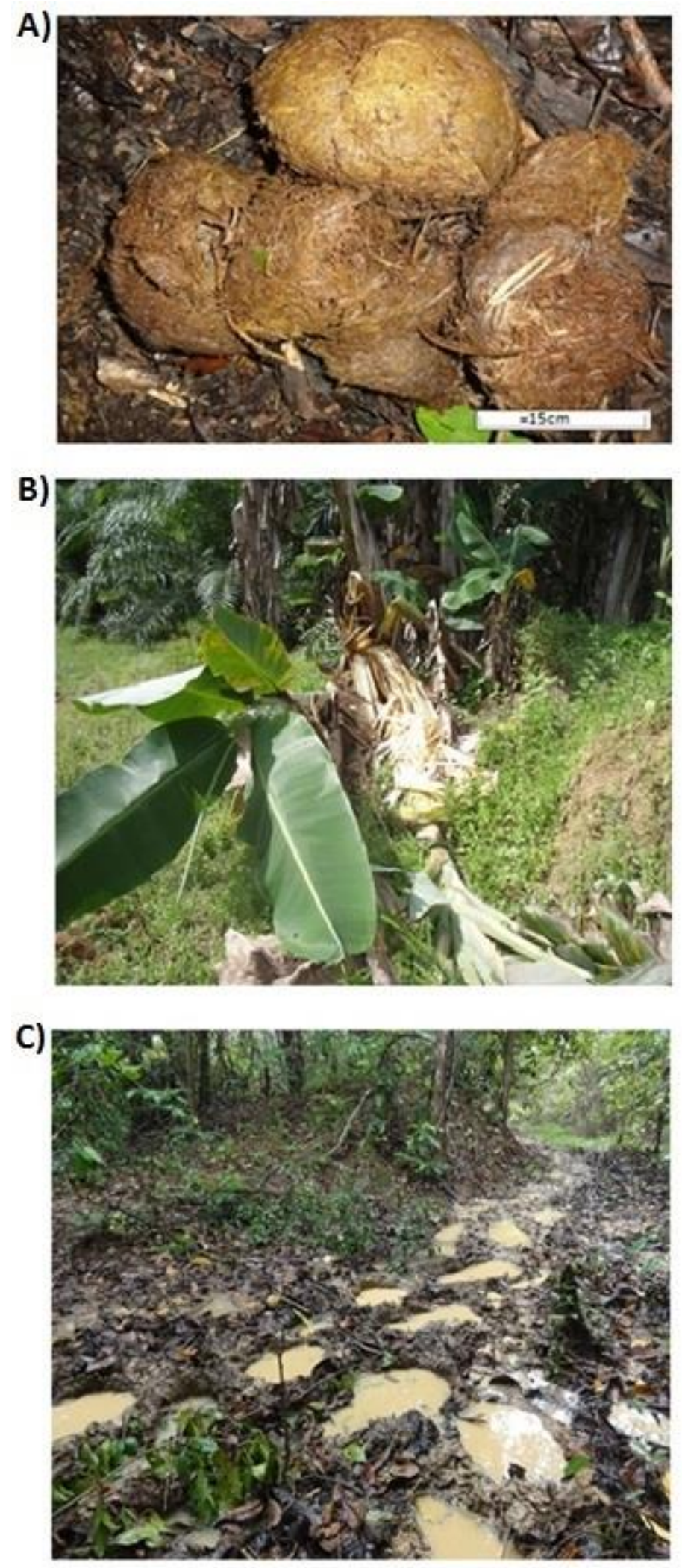

Figure 1. A) Elephant dung B) feed sign and C) footprints as measures of habitat use along $101 \mathrm{~km}$ randomly distributed transects, LKWS. 


\section{Results}

\section{a) Habitat type classification}

Variation between four habitat types: lowland mixed dipterocarp forest; semi-inundated dipterocarp forest; swamp forest and open grass areas along forest margins, was evident using MDS and PERMANOVA. Recently logged or cultivated areas were not included in the analyses but were categorised as a fifth habitat type. Semi-inundated dipterocarp forest and open grass areas had not previously been described quantitatively and discriminated from other habitats (Figure 2a and 3a). Permanova tests found significant variation in all environmental variables between the four different habitat types $\left(F_{1,3}=\leq 1.6, P=\leq 0.02\right)$ except elevation $(F=1,31.0, P=0.42)$ (i.e. ranged between $15 \mathrm{~m}$ and $32 \mathrm{~m}$ ) and understorey $\operatorname{cover}\left(F_{1,3}=\leq 1.6, P=0.06\right)$ (Table 1$)$. Floristic composition also varied significantly $\left(F_{1,3}=\right.$ $\leq 13.0, P=\leq 0.01$ ) amongst habitat types (Table 2). Similarity Percentage analysis identified that shade percentage and distance to water accounted for the greatest dissimilarity between habitat types (Figure 2b) and the presence of Poaceae, Cyperaceae, Leguminosae and Lauraceae accounted for the greatest dissimilarities in floristic composition between habitat types (Figure 3b).

Table 1. Results of PERMANOVA (permutation analysis of variance) examining the association of Environmental site characteristics and Habitat types. All environmental covariates were statistically significant except Elevation and Understory cover.

\begin{tabular}{lllllll}
\hline Source & df & \multicolumn{1}{c}{ SS } & MS & Pseudo-F & P(perm) & Unique perms \\
\hline Elevation & 1 & 2925 & 2925 & 1.0098 & 0.428 & 9948 \\
Water & 1 & 9640.6 & 9640.6 & 3.328 & 0.0017 & 9947 \\
Shade & 1 & 28151 & 28151 & 9.718 & 0.0001 & 9938 \\
Understorey & 1 & 5528.7 & 5528.7 & 1.9086 & 0.0642 & 9938 \\
Habitat & 3 & 18950 & 4737.6 & 1.6355 & 0.021 & 9886 \\
Residuals & 46 & $1.33 \mathrm{E}+05$ & 2896.8 & & & \\
Total & 53 & & & & & \\
& & & & & &
\end{tabular}


Table 2. Results of PERMANOVA (permutation analysis of variance) examining the association of plant family presence/absence and Habitat types. Statistically significant plant families between habitat types included Leguminosae, Poaceae, Lauraceae, Ebenaceae and Dillineaceae.

\begin{tabular}{lllllll}
\hline Source & df & SS & MS & Pseudo-F & P(perm) & $\begin{array}{l}\text { Unique } \\
\text { perms }\end{array}$ \\
\hline Leguminosae & 1 & 4486.4 & 4486.4 & 11.687 & 0.0008 & 9937 \\
Poaceae & 1 & 7984.9 & 7984.9 & 20.8 & 0.0001 & 9936 \\
Cyperaceae & 1 & 1095 & 1095 & 2.8523 & 0.0916 & 9927 \\
Zingiberaceae & 1 & 259.06 & 259.06 & 0.67483 & 0.4437 & 9927 \\
Arecaceae & 1 & 658.45 & 658.45 & 1.7152 & 0.1865 & 9942 \\
Euphorbiaceae & 1 & 740.26 & 740.26 & 1.9283 & 0.1537 & 9932 \\
Ebenaceae & 1 & 1842.2 & 1842.2 & 4.7986 & 0.0243 & 9933 \\
Lauraceae & 1 & 2788.1 & 2788.1 & 7.2626 & 0.0073 & 9939 \\
Myrtaceae & 1 & 190.4 & 190.4 & 0.49597 & 0.516 & 9919 \\
Dillineaceae & 1 & 5988.9 & 5988.9 & 15.6 & 0.0004 & 9928 \\
Habitat & 3 & 20097 & 5024.2 & 13.088 & 0.0001 & 9952 \\
Residuals & 40 & 15356 & 383.89 & & & \\
Total & 54 & & & & & \\
\hline
\end{tabular}




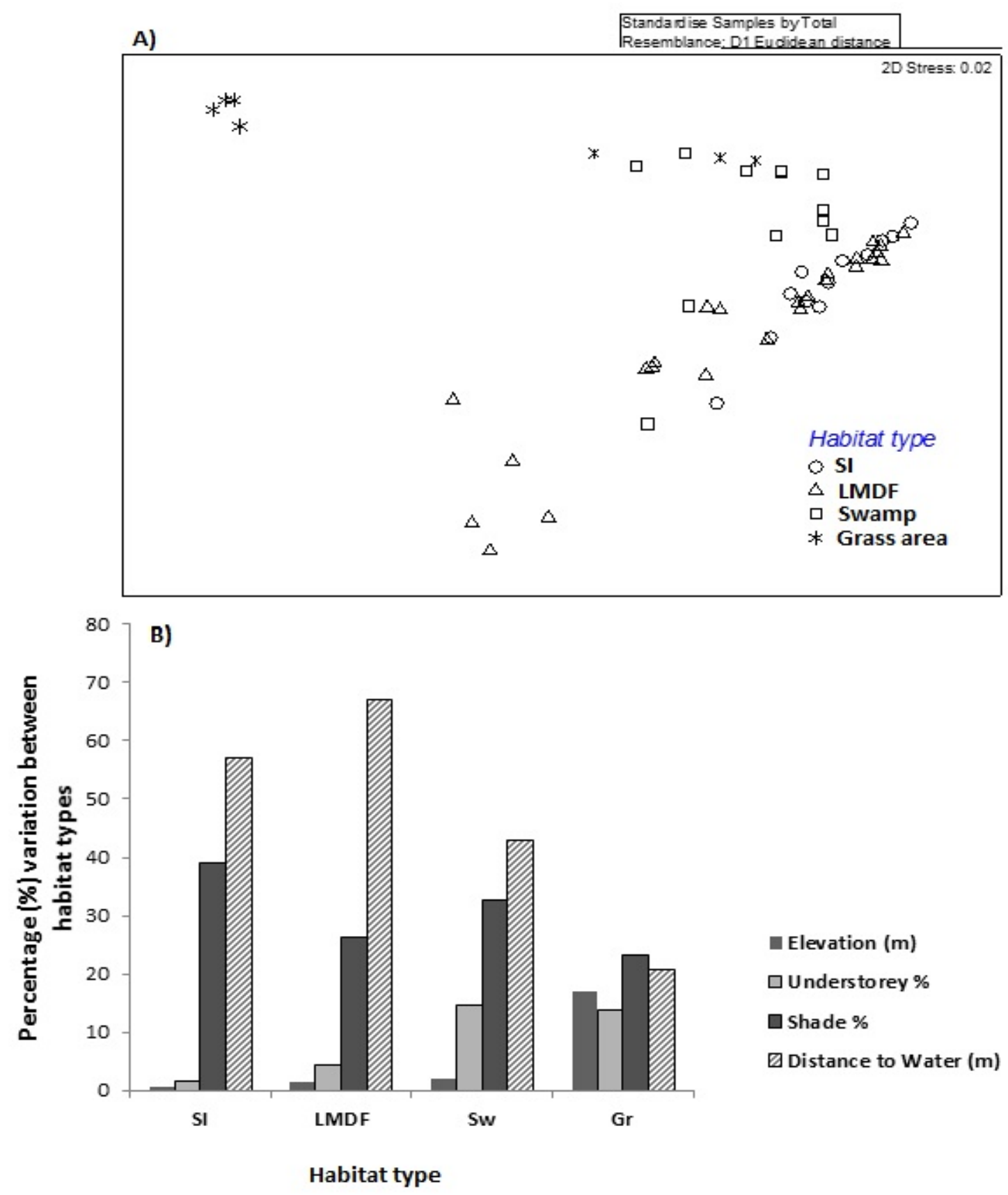

Figure 2. A) Multidimensional scaling ordination plot for environmental variables and habitat type as the factor B) Similarity Percentage Analysis (SIMPER) determined the percentage of each environmental variable responsible for the most dissimilarity between habitat types $(\mathrm{SI}=\mathrm{Semi}$-inundated, $\mathrm{LMDF}=$ lowland mixed dipterocarp, $\mathrm{Sw}=\mathrm{Swamp}, \mathrm{Gr}=$ Grass area) and elevation (m), distance to water (m), understorey density (\%) and shade (\%) as environmental variables. 

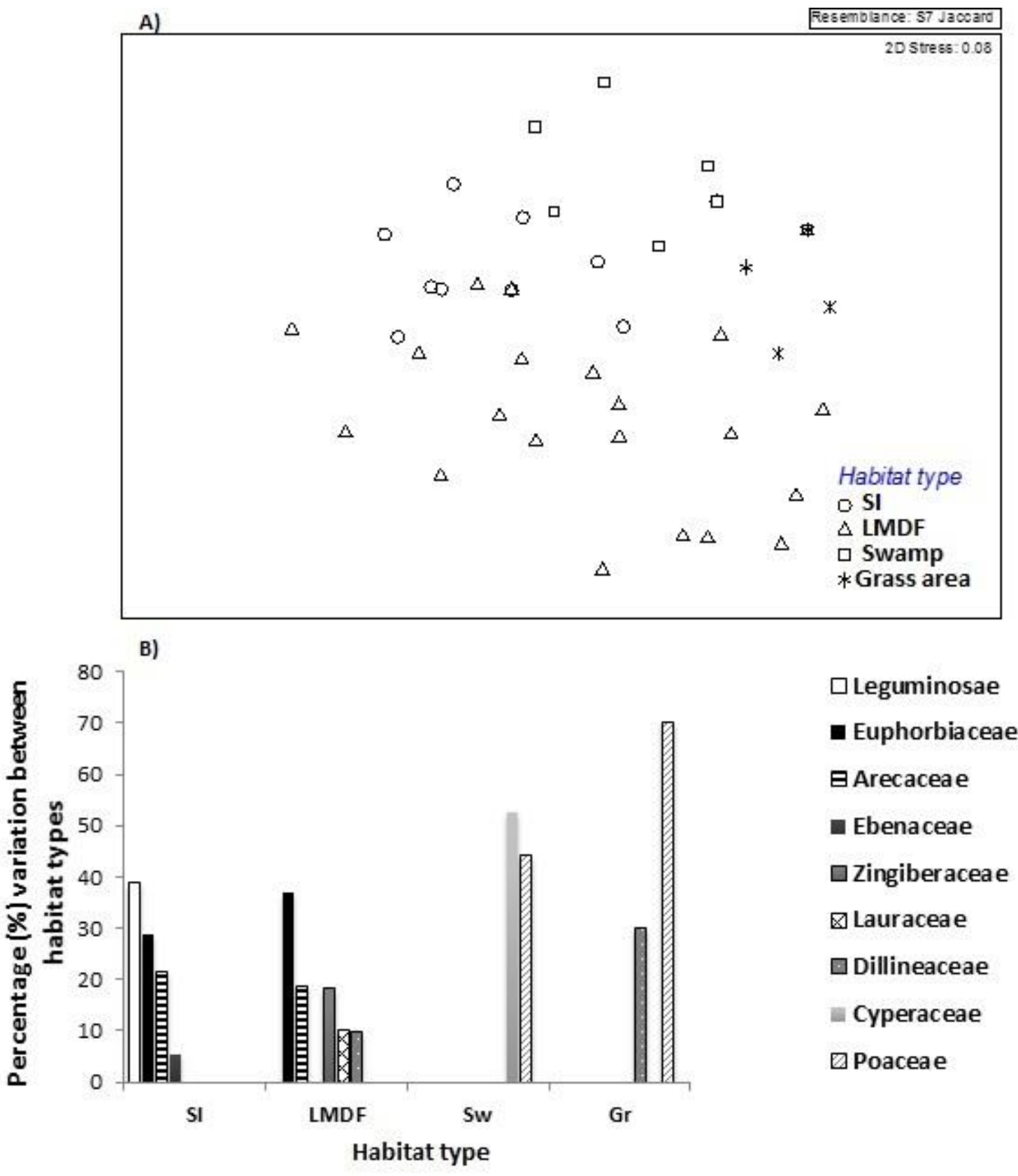

Figure 3. A) Multidimensional scaling plot ordination with habitat type as the factor and floristic composition as biological variables (Leguminosae, Arecaceae, Euphorbiaceae, Myrtaceae, Dillineaceae, Poaceae, Cyperaceae, Lauraceae and Ebenaceae). B) Similarity Percentage Analysis (SIMPER) determined the percentage of plant families responsible for most dissimilarity between habitat types. 


\section{b) Habitat availability}

Habitat type availability measures identified that lowland mixed dipterocarp forest (LMDF) is the most common habitat type within $1 \mathrm{~km}$ of the Kinabatangan River in LKWS (34\%) (Figure 4), followed by swamp (22\%) (Figure 5) and semi-inundated dipterocarp forest $(21 \%)$ (Figure 6). Open grass areas (14\%) (Figure 7) and recently logged or cultivated (11\%) (Figure 8) are less common (see also Figure 11).

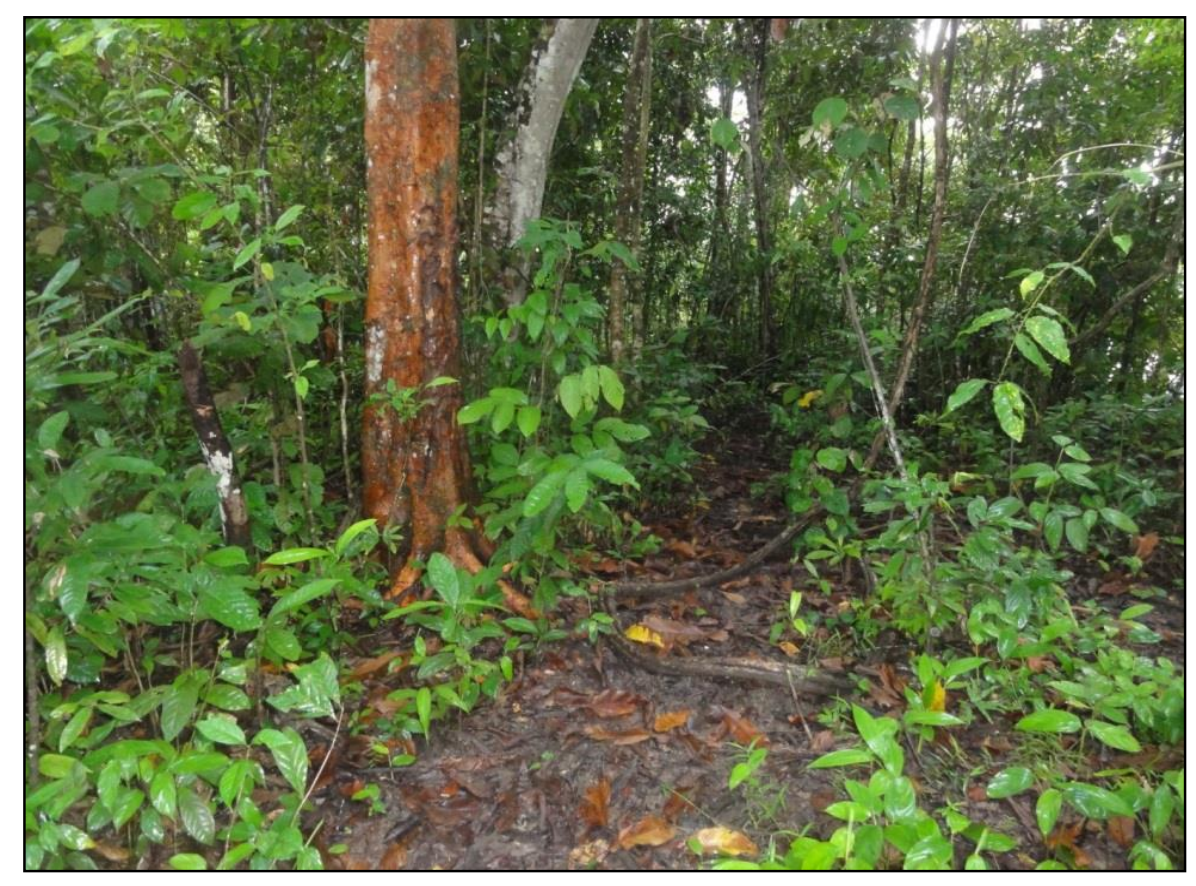

Figure 4. Lowland mixed dipterocarp forest in LKWS. 


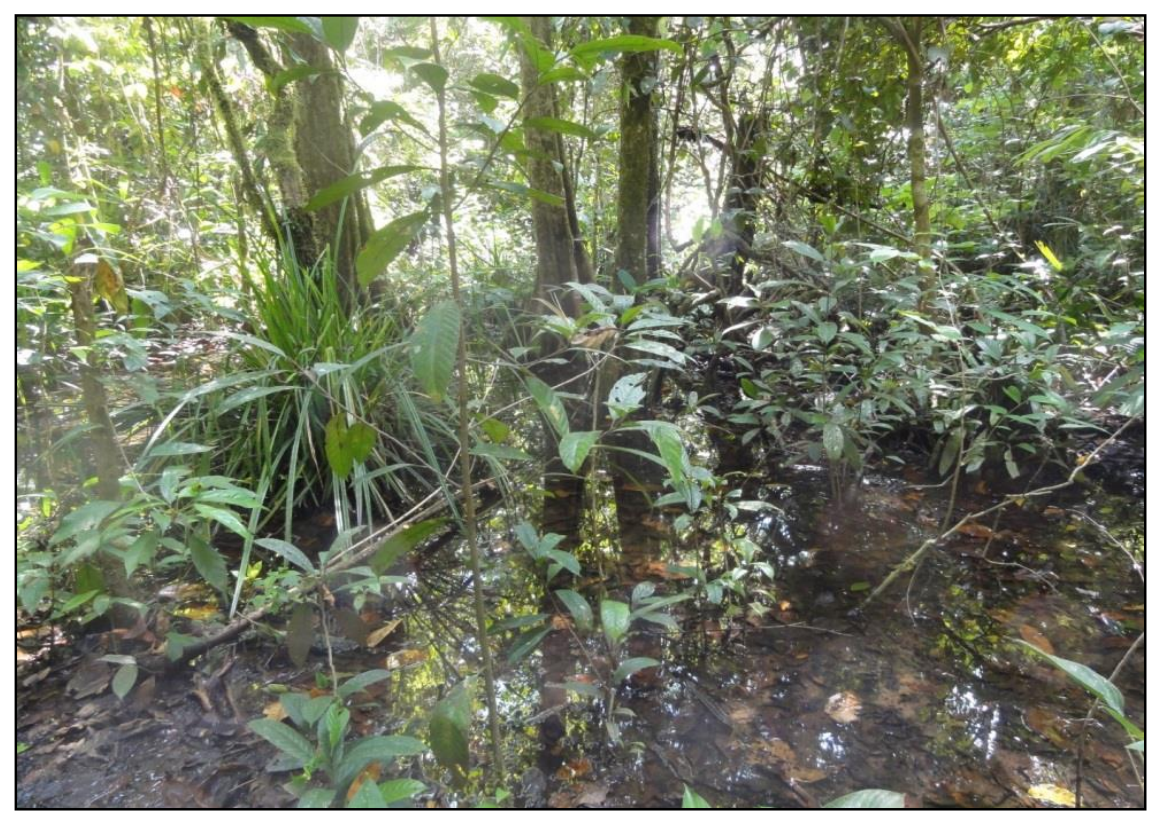

Figure 5. Semi-inundated dipterocarp forest in LKWS.

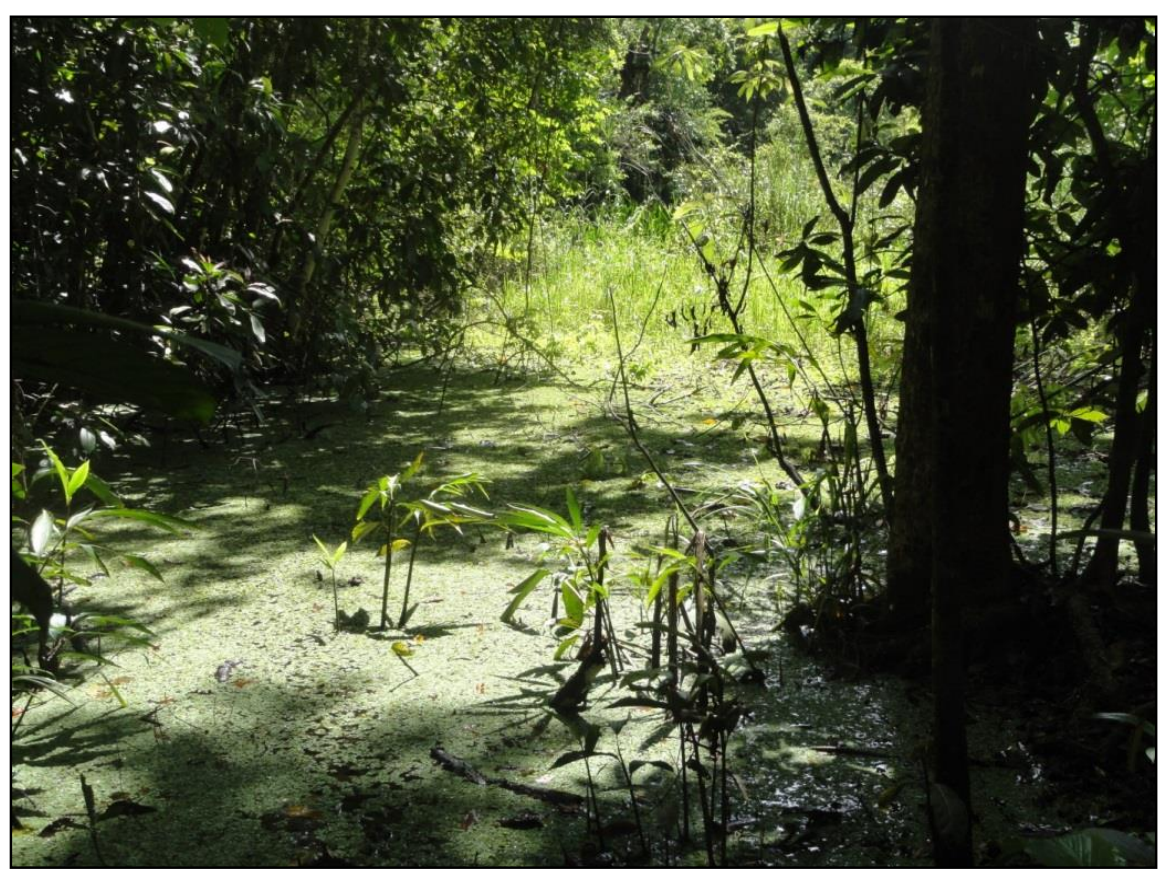

Figure 6. Swamp in LKWS 


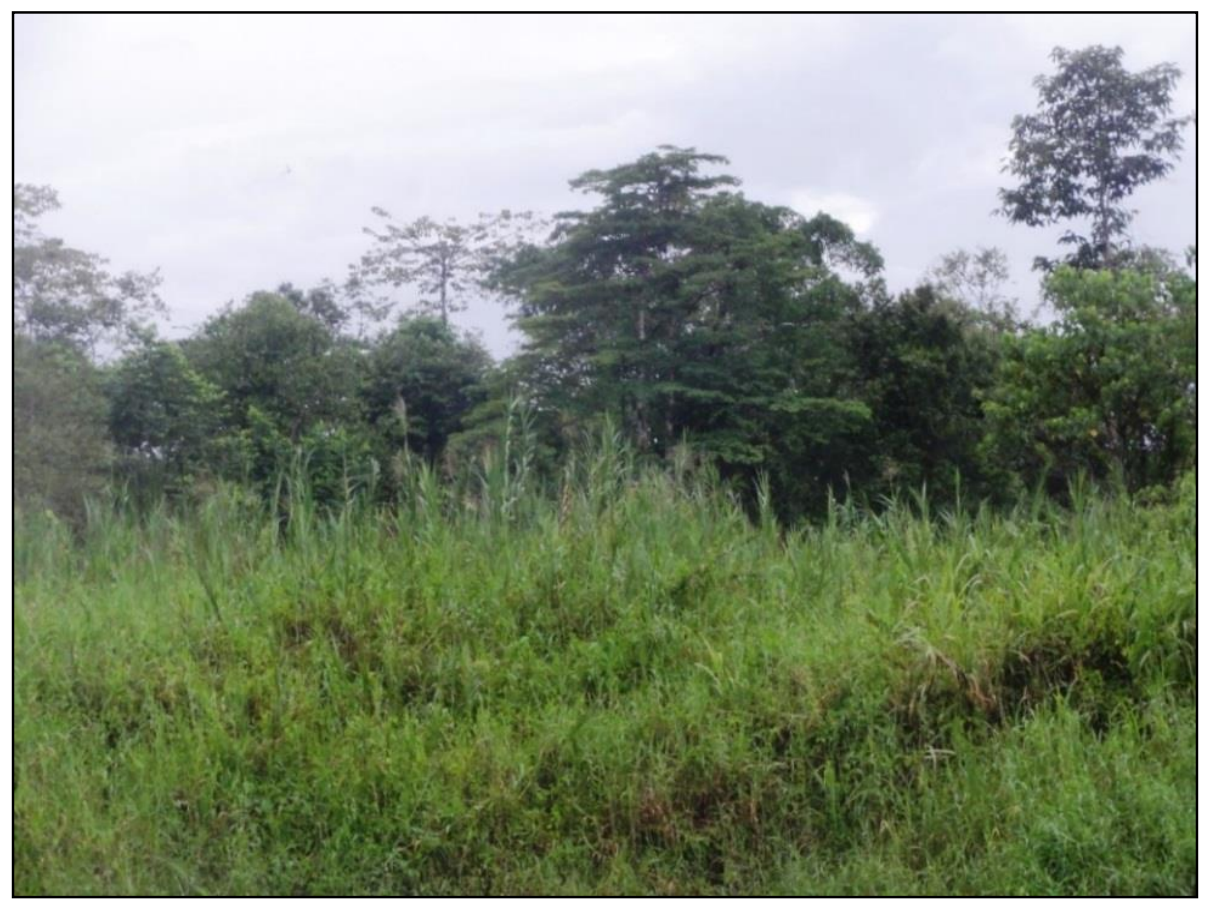

Figure 7. Open grass area in LKWS

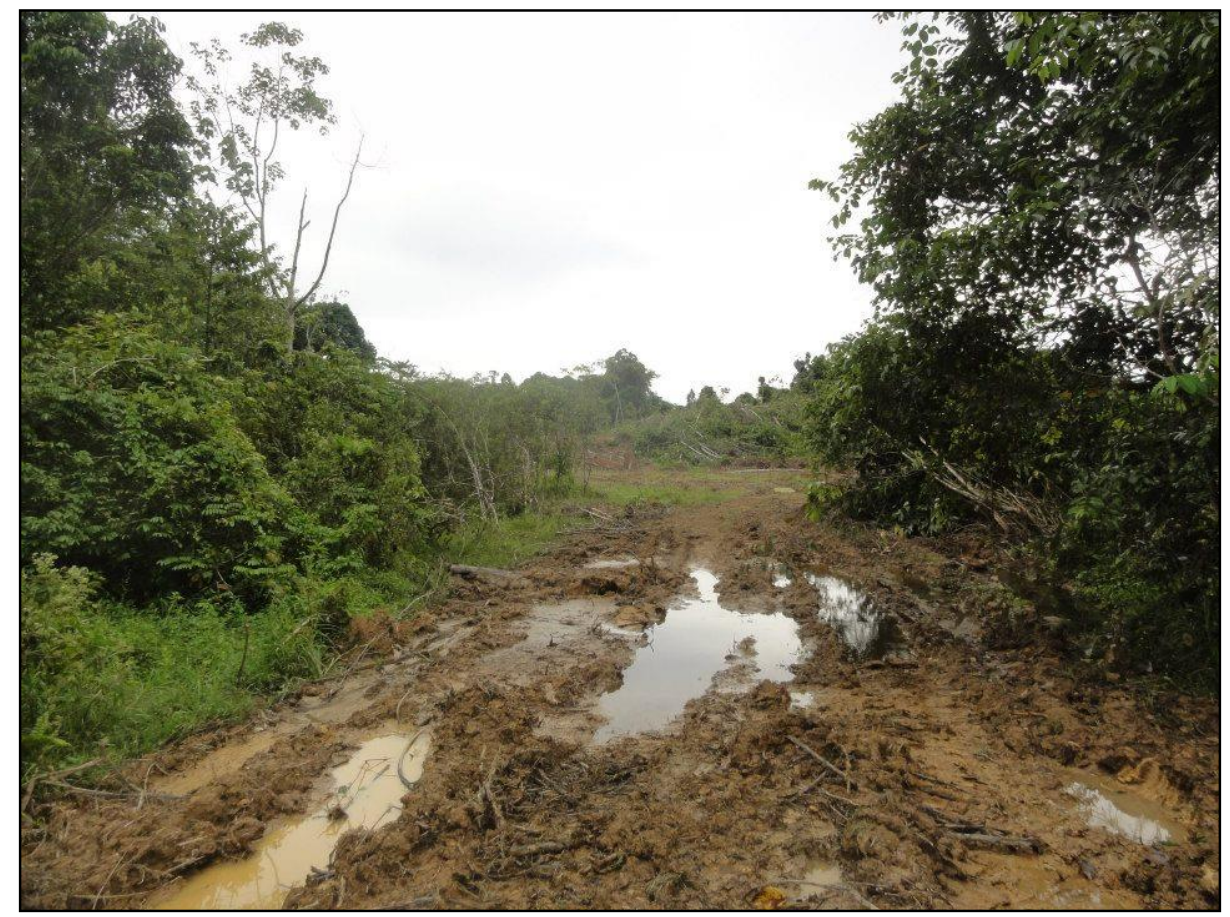

Figure 8. Recently logged habitat in LKWS 


\section{c) Elephant habitat use for feeding}

The relative frequencies of the three types of elephant sign - dung piles $(n=48)$, footprints $(n=61)$, and feeding sign $(n=47)$ - varied between the five habitat types (Figure 9).

Footprints were the most common sign in drier habitats (open grass areas, LMDF, and logged or cultivated areas) but feed sign was more commonly found as evidence of elephant activities in semi-inundated and swamp habitats. Dung and footprints were only $42 \%$ of all sign in swamp and 56\% in semi-inundated but were $90 \%$ in open grass areas and recently logged or cultivated. LMDF was $72 \%$. We would expect water to obscure dung and footprints and to accelerate their decay and destruction (Barnes et al., 1995). The pattern in detection across habitat types met my expectations that water would reduce my ability to detect ground-based sign and would introduce a negative bias in semi-inundated and swamp habitats. The reduced presence of water in semi-inundated habitat appeared to also result in an intermediate condition between swamp and drier habitats.

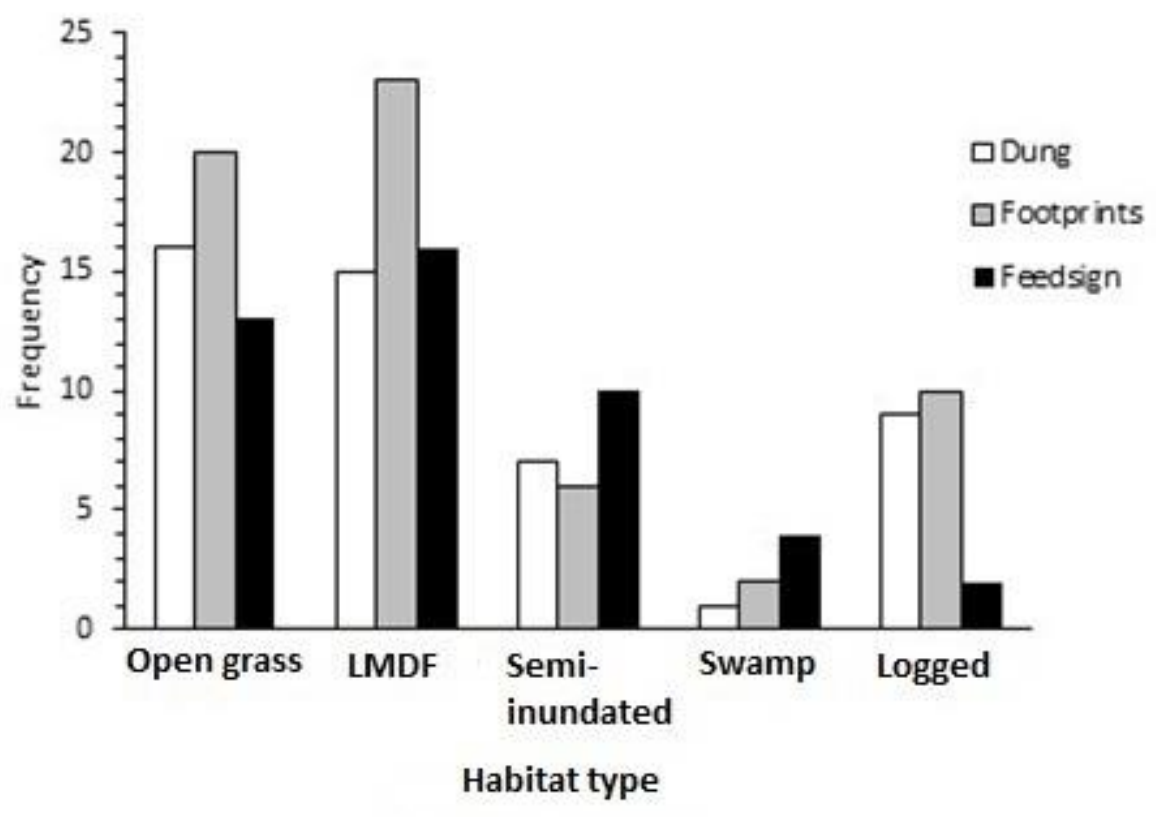

Figure 9. Frequency of elephant signs in each habitat type recorded along10 randomly distributed $1 \mathrm{~km}$ transects (LMDF=lowland mixed dipterocarp forest). 
The distances between dung and nearest feed $\operatorname{sign}(n=35$, average $=17 \mathrm{~m}$, median $=10 \mathrm{~m}$, $\mathrm{SD}=4.38)$, and footprints and nearest feed sign $(\mathrm{n}=39$, average $=17 \mathrm{~m}$, median $=12 \mathrm{~m}, \mathrm{SD}=$ 5.42) were significantly less than the distances between a random selection of distances between feed sign and nearest-neighbour feed sign (average $=90 \mathrm{~m}$ and median $=62 \mathrm{~m}, \mathrm{t}-$ test, $\mathrm{df}=34, \mathrm{P}=<0.01$ (Figure $10 \mathrm{~A}$ ) and average $=95 \mathrm{~m}$ and median $=63 \mathrm{~m}, \mathrm{t}$-test, $\mathrm{df}=37$, $\mathrm{P}=<0.01$ ) (Figure 10B). Thus, dung and footprints were spatially associated with feed sign and so the latter was used in the habitat-use analyses as a reliable indicator of use across all habitat types where year-round wet conditions prevent ground-deposited sign from being reliably detected or persistent.

There was a significant difference between elephant habitat use for feeding (feed sign) and habitat availability (Chi-square, $\mathrm{N}=147, P=<0.05$ ) (Figure 11). Lowland mixed dipterocarp forest and semi-inundated dipterocarp forest were used in proportion to their availability, whereas recently logged or cultivated was used half as much and swamp a third as much as availability. The elephants selected open grass areas more than double the availability, indicating a preference for this habitat type. 


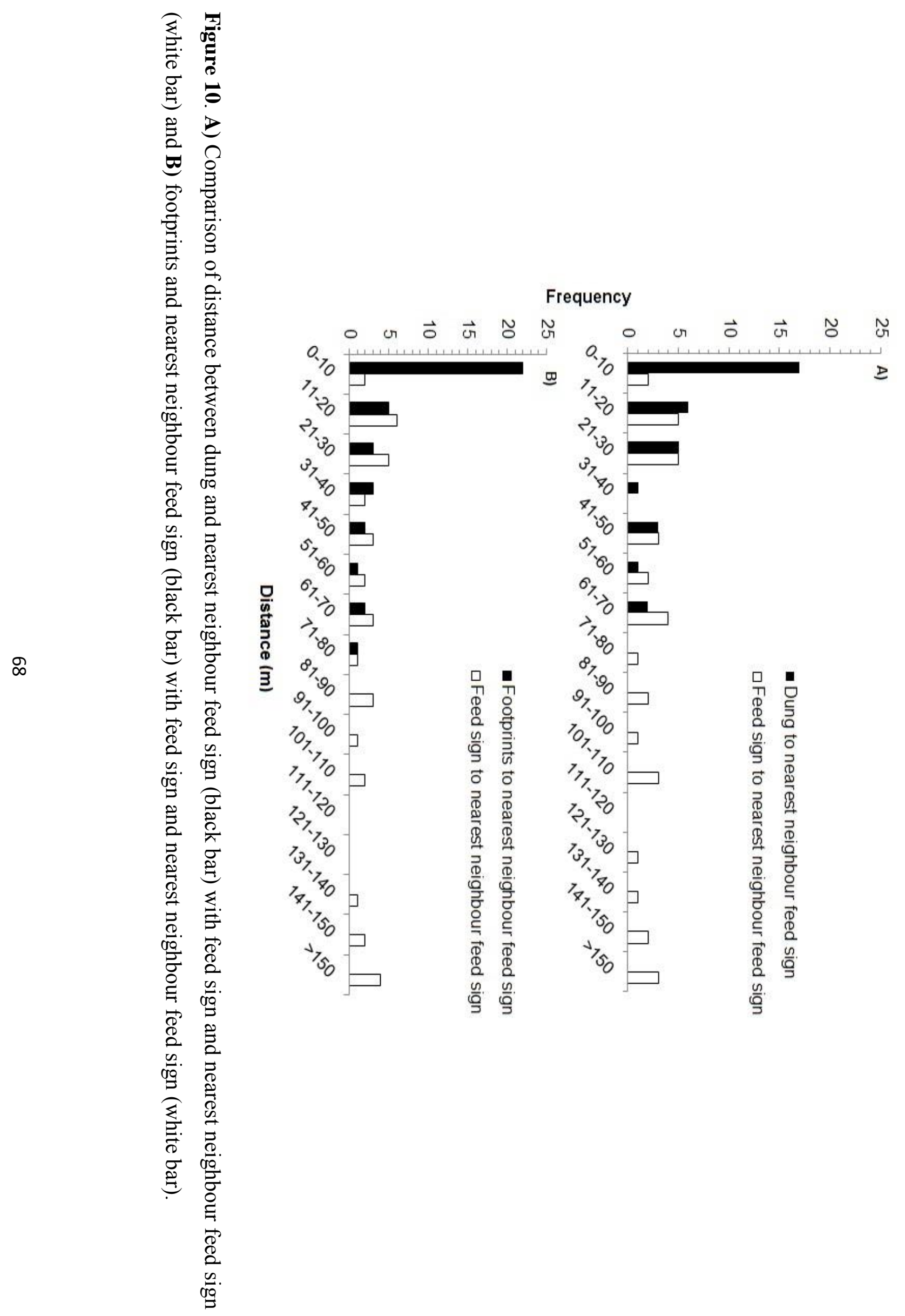




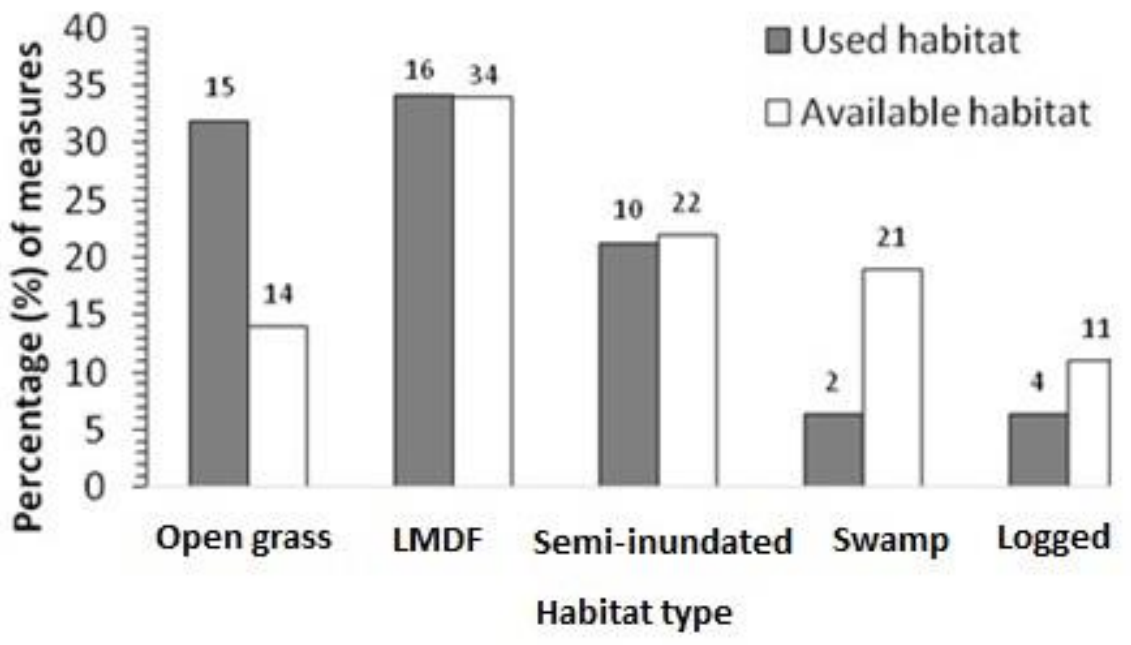

Figure 11. Percentage of available habitat types $(n=100)$ and habitat use for feeding by elephants $(n=47)$. Sample sizes are shown above bars. (LMDF=Lowland mixed dipterocarp forest).

\section{Discussion}

Five habitat types utilised by elephants are described in the LKWS. Two habitat types, lowland mixed dipterocarp forest and swamp, had been described in a report published by World Wildlife Fund, Malaysia (Azmi, 1998). Two others had been referred to anecdotally but had not been described or quantified as separate habitat types: open grass areas and semiinundated dipterocarp forest. The fifth habitat type: recently logged or cultivated, may have been any of the above habitat types at some point but at the time of observation had been cleared for cultivation, logging, human habitation or infrastructure.

LMDF was the most common habitat type with semi-inundated forest and swamp ranking second and third most common. LMDF was characterised by high shade cover and presence of woody species (Euphorbiaceae, Lauraceae, Myrtaceae, Ebenaceae, Dillineaceae and Leguminosae) and early successional species like Zingiberaceae. Semi-inundated forest 
was characterised by low understorey density, presence of palms (Arecaceae), lianas (Leguminosae) and woody species (Myrtaceae and Euphorbiaceae). This habitat type also retained water for longer after rainfall compared to LMDF. Swamp was comprised of permanently or seasonally inundated forest containing plant species tolerant of high water tables. Vegetation varied between open herbaceous swamps and swamp forests. Swamp forest was generally low in stature with an irregular open canopy. Trees include those from the families Myrtaceae and Euphorbiaceae. Understorey in swamps was either sparse or dense with species commonly from the families Cyperaceae and Poaceae.

Open grass areas, including within riverine areas as well as inland grass patches along forest margins, is formed by a combination of natural hydrological river processes as well as man-made forest clearing and natural forest disturbance. It contained a variety of species in Poaceae, predominantly Phragmites sp., Pennisetum sp. and Dinochloa sp. Canopy cover was minimal, resulting in prevalence of early successional species such as those from Zingiberaceae. Understorey cover was high. Woody species from the family Dillineaceae were common. Recently logged or cultivated habitat represented areas with recent or ongoing human disturbance such as near villages, roads and cultivation. There was minimal natural growth with the exception of some early successional species and cultivated crops. This habitat type was ranked last but proved to be a surprisingly large minority (11\%) given that transects were located from the Kinabatangan River and confined to within $1 \mathrm{~km}-$ an indication of ongoing encroachment of agriculture into elephant habitat in the region. Importantly, elevation was one habitat characteristic that did not influence the habitat types. Canopy cover and soil composition are probably the major influences on vegetation composition and structure in the LKWS jungle, although soil composition was not measured in this study.

Previous studies of elephant habitat use have relied on direct observations of elephants (Kumar et al., 2010; Shrader et al., 2012), presence of dung (Wilson et al., 2013), or positions of elephants recorded via satellite tracking (DeBoer et al., 2005). At the time this research took place no detailed vegetative map of the sanctuary was available, therefore determining habitat use by using elephant locations via satellite tracking was not possible. On-ground measures were therefore necessary to establish the characteristics of habitat types as well as their use by elephants. Feed sign proved to be a useful proxy for elephant habitat use because its presence was associated strongly with the other forms of spoor and sign recorded (dung and footprints) that were less reliable where soil moisture conditions, especially periodic or permanent flooding, introduce a negative detection bias in some 
habitats; for example, swamps and semi-inundated habitat. Arguably, feed sign is also the more appropriate metric of elephant habitat use because it indicates habitat use for foraging and excludes habitat use for travelling that may be a disproportionate part of habitat use in fragmented forest landscapes like the LKWS but not indicative of the value of the habitat to elephants.

The elephants in LKWS showed a relatively strong preference for just one habitat type: the patches of open grass areas that are typically located along the riparian zone of the Kinabatangan River and inland forest margins. LMDF, the most common habitat type, was also frequently used by the elephants but, along with semi-inundated dipterocarp forest, it was used in proportion to its availability. Thus LMDF constitutes a substantial portion of the total habitat used by elephant but it was not preferred. Semi-inundated habitat was the third most used habitat type and was neither preferred nor avoided. Swamp on the other hand, was an avoided habitat type, as were areas that had been recently logged or cultivated.

Like this study, earlier studies have found Asian elephants to prefer riparian and forest margin ecotone areas but avoid areas of ongoing human disturbance (Kumar et al., 2010). Riparian zones and forest margins are areas at an interface between different vegetation communities and do not necessarily represent a specific habitat type as such but rather a zone comprised of a number of habitat types. Nevertheless, such zones are comparatively biodiverse. Moreover, in the LKWS they are most often an interface between forest and small patches of open grass areas that are at the edges of rivers and forest. Thus, riparian and forest margins may have several properties attractive to elephants such as access to water and a diverse range of food plants that grow within the forest edge beneath an enclosed canopy and others that flourish in open areas alongside these margins, such as early successional plants like grasses, gingers and bamboos. These open areas may also provide ease of movement compared to movement within the forest or swamps.

Studies of other forest elephant taxa (Loxodonta cyclotis) (Blake, 2002) and (Elephas maximus) (Sukumar, 1990) describe forest elephant preference for a browse diet and therefore habitat types containing these browse species, unless grass was the dominant vegetation. On the other hand, Tchamba \& Seme (1993) and Olivier (1978) found grasses to be the preferred diet of forest elephants despite grass areas being less abundant. In LKWS, although open grass areas cover just $14 \%$ of the landscape (compared to $56 \%$ forested areas and $21 \%$ swamp) this habitat was preferred.

For the purposes of my study it was necessary to identify the habitat types utilised by the elephants and the habitat composition including the habitat types that make up the 
riparian zone. Without specific classification of open grass areas and semi-inundated forest in the LKWS their importance to various species, including elephants, cannot be understood. Moreover, many open grass areas, regardless of whether their origin is natural or augmented by logging, are believed to impede movement, dispersal and social activities of other species such as proboscis monkeys (Nasalis larvatus) (Sha et al., 2008). Patches of open grass areas along the river may also be considered less visually appealing to tourists visiting the area to observe wildlife from boats along the Kinabatangan River. In recent years attempts have therefore been made to reforest many of these areas to improve forest connectivity for wildlife, particularly primates, and to improve visual appeal for the tourism industry.

My results have identified and described a habitat type of importance to the elephant population in LKWS - open grass areas - as well as provided quantitative characterisation of established categories. Furthermore, the information gained in this study will be used to inform the remaining chapters that explore the influence of floristic composition and physical characteristics of habitat types amongst foraging sites on finer-scale elephant resource-use, such as the use of sites and forage plants. Understanding the habitat preferences and, in later chapters, the qualities of the habitats that influence elephant spatial and temporal use of foraging sites, can foster and inform management strategies for the elephant population by identifying key areas and resources for the species. If the elephant population depends on open grass areas, which are scattered and few across the landscape, in order to meet their resource needs, then conservation planners must incorporate these areas into a landscape management strategy. 


\section{Chapter Four}

Foraging site recursion by forest elephants

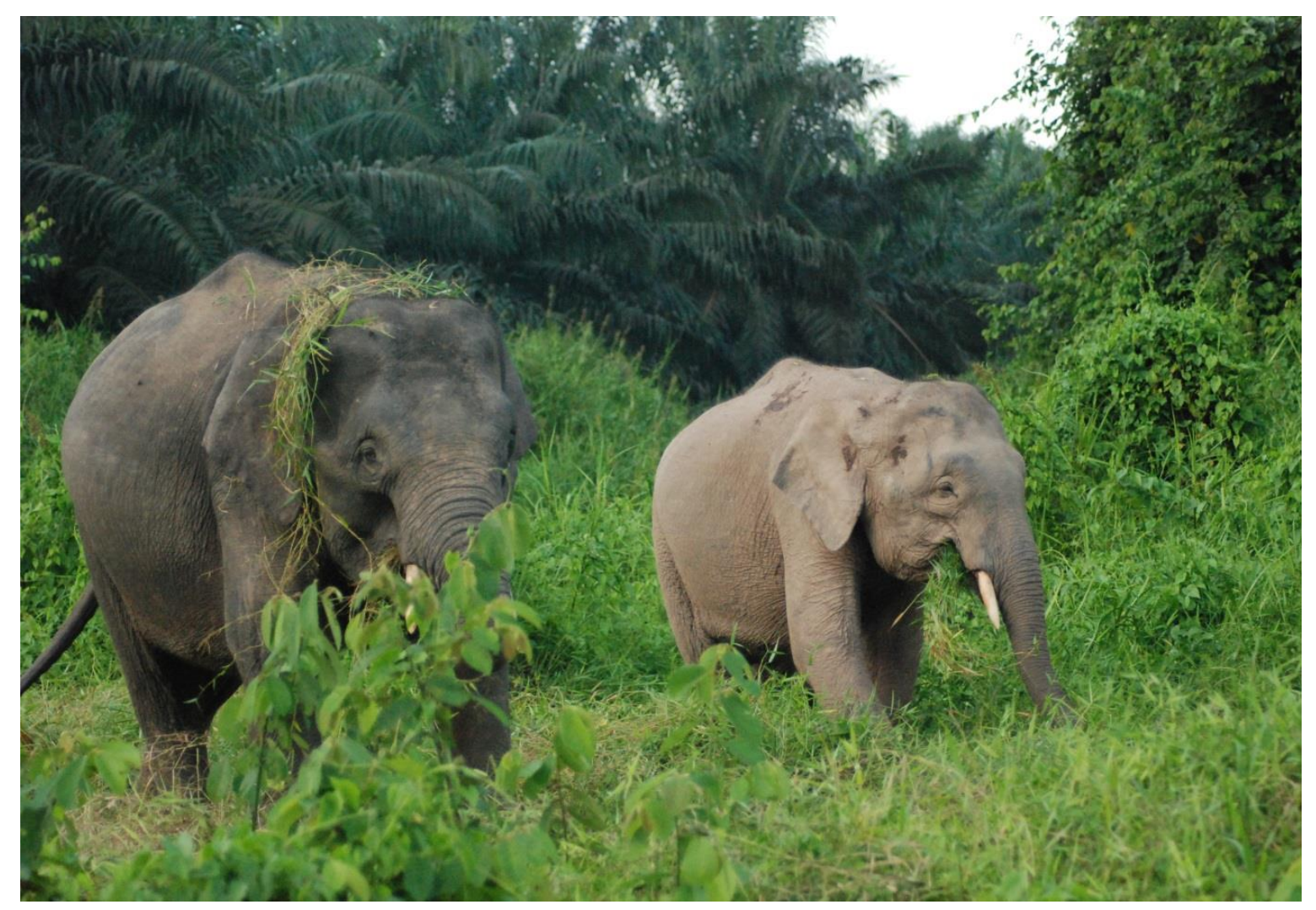

Sub-adult males feeding in an open grass area alongside an oil palm plantation, Lower Kinabatangan, Sabah, 2010

Authors note: Chapter Four was published in the journal Current Zoology in a special edition on Behaviour and Conservation in July 2014 (Appendix Four). 


\begin{abstract}
Recursion by herbivores is the repeated use of the same site or plants. Recursion by wild animals is rarely investigated but may be ubiquitous. Optimal foraging theory predicts site recursion as a function of the quality of the site, extent of its last use, and time since its last use because these influence site resource status and recovery. We used GPS collars, behaviour and site sampling to investigate recursion to foraging sites for the two main elephant (Elephas maximus borneensis) herds in the Lower Kinabatangan Wildlife Sanctuary, Borneo, over a 12 month period. Recursion occurred to 48 out of 87 foraging sites and was most common within 48 hours or between 151-250 days, indicating two different types of recursion. Recursion was more likely to occur if the site had previously been occupied for longer. Moreover, the time spent at a site at recursion was the same as the time spent at the site on the previous occasion. The number of days that had passed between the first visit and recursion was also positively correlated with how much time was spent at the site at recursion. Habitat type also influenced the intensity of site-use, with more time spent at recursion within open grass areas along forest margins compared to other habitat types. Recursion is a common behaviour used by the elephants and its pattern suggests it may be a foraging strategy for revisiting areas of greater value. The qualities of recursion sites might usefully be incorporated into landscape management strategies for elephant conservation in the area.
\end{abstract}




\section{Introduction}

Understanding how animals utilise and navigate a landscape is essential to comprehending species ecological and population processes (Stephens \& Krebs, 1986; Turchin, 1991; Bartumeus et al., 2005). Feed quality and the spatial distribution of foraging sites may influence foraging strategies of herbivores and the way in which they utilise the landscape (Owen-Smith \& Novellie, 1982; Belovsky, 1984; Pyke, 1984; Senft et al., 1987; Adler et al., 2001). Recognising foraging patterns across the landscape has benefits for species and habitat conservation. The spatial and temporal variation in species foraging behaviour and habitat utilisation may be incorporated into landscape management strategies and habitat assessment.

The return by animals to previously utilised foraging sites is known as recursion. Studies of recursion are few (Bar-David et al., 2009; Li et al., 2012; Riotte-Lambert et al., 2013). However, studies of animal behaviours that resemble recursion have been explored more extensively. Those studies used different terminology to recursion but have explored a similar process. Examples of foraging recursion include; the influence of spatial memory and resource relocation in bees (Kadmon, 1992; Williams \& Thomson, 1998; Stout \& Goulson, 2002; Menzel et al., 2005), nectar feeding birds returning to flowers (Gill, 1998; Burke \& Fulham, 2003), sheep and cattle returning to grass patches (Bailey et al., 1989; Dumont \& Petit, 1998) and primates returning to fruit trees (Garber, 1988; Janson, 1998; Watts, 1998; Garber \& Jelinik, 2006; Erhart \& Overdorff, 2008; Janmaat et al., 2013; Porter \& Garber, 2013).

Temporal pattern in site revisits have been related to resource recovery, for example in insects (Bell, 1990; Williams \& Thomson, 1998) and birds (Motacilla sp.) (Davies \& Houston, 1981). The predictability and order of repeated visits to previously utilised sites has been explored extensively in bumblebees (Bombus sp.) (Comba, 1999; Heinrich, 1976; Manning, 1956; Thomson, 1996; Thomson et al., 1982, 1987), euglossine bees (Euglossini spp.) (Janzen, 1971; Ackerman et al., 1982; Dressler, 1982), butterflies (Danaus sp.) (Gilbert, 1980), hummingbirds (Phaethornis spp.) (Gill, 1988; Tiebout, 1991; Garrison \& Gass, 1999), wagtails (Motacilla sp.) (Davies \& Houston, 1981), bats (Glossophaga sp., Pippistrellus sp.) (Lemke, 1984; Racey \& Swift, 1985), and primates (Saguinus spp., Cebus sp., Gorilla sp.) (Garber, 1988; Janson, 1998; Watts, 1998). Additionally, the influence of resource renewal on site reuse has been explored using simulations of animal movement patterns (Possingham, 1989; Ohashi \& Thomson, 2005; Bar-David et al., 2009). Nevertheless, only some studies 
have explored foraging site reuse in uncontrolled environments and these studies are largely limited to primates (Garber, 1989; Watts, 1998; Garber \& Jetlinik, 2006; Erhart \& Overdorff, 2008; Janmaat et al., 2013; Porter \& Garber, 2013) and a few ungulates (Syncerus sp., Aepyceros sp.) (Bar-David et al., 2009; Riotte-Lambert et al., 2013).

Habitat quality and its importance to animals, or individual resource quality and its importance within foraging sites, might be best understood by measuring the amount of time animals spend at these sites and how frequently they return to them. Single point-in-time surveys, designed to identify selected resources or avoided habitat features, that do not take into account temporal variation in resource-use might under- or over-estimate the relative importance of sites visited and their resource characteristics. If an animal repeatedly visits a site and spends more time at a site relative to others, this may help to identify high quality areas or more critical resources.

We investigated foraging site recursion by a wild population of Bornean elephants (Elephas maximus borneensis) and the foraging mechanisms that might explain recursion behaviour. Our aims were to identify if recursion occurs, how often, and what environmental and biological variables may influence this behaviour. In an optimally foraging herbivore, recursive site-use should be a function of the intensity of last use. Recursion should also relate to time since last use to allow for resource recovery after depletion (Ohashi \& Thomson, 2005). The composition of the site should influence recursion because a herbivore will have specific resource requirements and site quality and recovery rates may vary with endogenous (for example, nutrient content) and exogenous (for example, climatic) influences. Recursion behaviour may also, however, be an indicator of diminishing habitat of suitable quality or capacity because, as prime habitat becomes less available, recursion frequency should increase but time spent at sites decline. However, over time we would expect an optimally foraging herbivore to avoid unprofitable areas.

We expected elephants to spend more time at recursion sites per visit compared to non-recursion sites if the former were higher quality. Additionally, we expected elephants to return to sites at two temporal scales. Longer recursion times should occur in order for resources to sufficiently recover, especially where the site was used more extensively previously. Short-term recursions should occur as part of a site-sampling strategy (OwenSmith, 2002). We also expected site characteristics, such as habitat type, distance to water, distance to human habitation, shade and understorey cover to influence recursion and the intensity of site use. We expected more intensive use of sites within habitats containing preferred food plants of the Bornean elephant, such as within open grass areas along forest 
margins (English et al., 2014c), sites closer to a water source (McKay, 1973; Sukumar, 1990), sites further from human habitation (Blake, 2002) and sites less shaded (Barnes et al., 1995; Powell, 1997) allowing for more understorey growth, in particular grasses and bamboos (McKay, 1973; Shoshani \& Eisenberg, 1982).

\section{Methods}

\section{Study site and focal species}

This study focused on the area between the villages of Abai and Batu Puteh $\left(5^{\circ} 18^{\prime}-\mathrm{N} 5^{\circ} 42^{\prime}\right.$ $\mathrm{N}, 117^{\circ} 54^{\prime}-\mathrm{E} 118^{\circ} 33^{\prime}-\mathrm{E}$ ), which were the downriver and upriver limits of the LKWS elephant population's range. The study area contains 7 sections, each section referred to as a 'lot' (approximately $218 \mathrm{~km}^{2}$ ), including $89 \mathrm{~km}^{2}$ of protected forest reserves (Estes et al., 2012). The elephant herds utilised their whole range throughout the year including use of privately owned forests and cultivated land, particularly oil palm plantations that were adjacent to and between forested areas. Elephants in LKWS are mostly restricted to the linear fragments of forest along the Kinabatangan River (Estes et al., 2012) (Figure 1). See general Introduction, pages 26-28 for more detail of study site and focal species. 


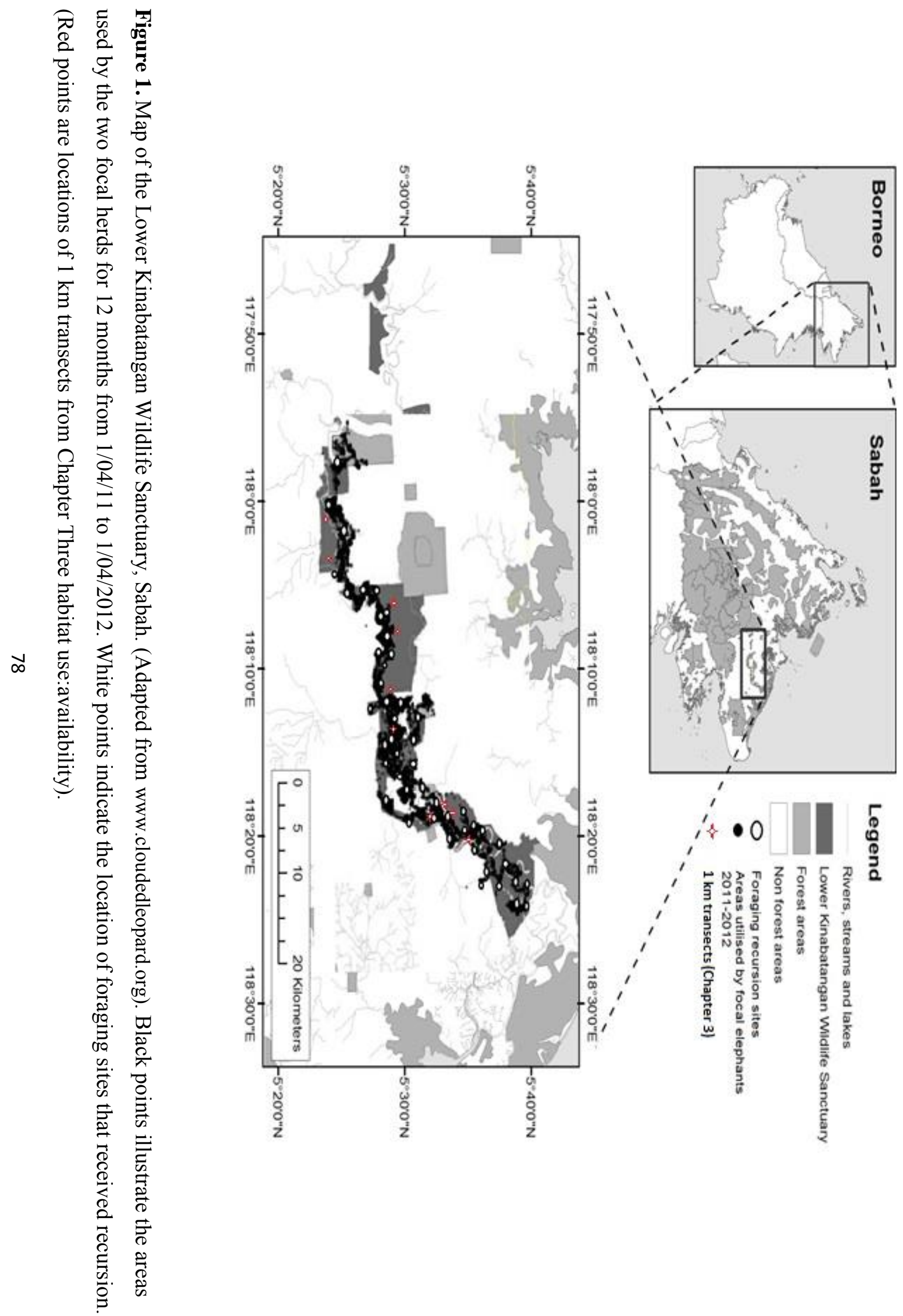




\section{Site location}

Two adult female elephants from the two main herds of the population in LKWS, each consisting of approximately 20-30 individuals, were randomly selected to attach a collar with a GPS transmitter in 2011. Neither female was the matriarch of her herd. Positions were recorded at hourly intervals for 12 months from April 2011 to May 2012. We identified and visited sites used by the elephants in April and May 2011, the early- to mid-dry season, and measured recursion to these sites over 12 months. Sites were distributed throughout their range with one site identified and measured per day. All sites were $>300 \mathrm{~m}$ apart. To identify sites used by the elephant herds, the latest GPS position recorded of each group on the days of observation was located and then fresh elephant sign, (footprints, dung, feed sign and vocalisations) was tracked until the focal female and her herd were located.

Once an elephant group was sighted we positioned ourselves at a distance so as not to disturb them. The activity of the majority of elephants at the site at the time of observation was recorded as either foraging, resting or moving. Moving sites were those where the focal female and her herd were seen walking without stopping to feed. Resting sites were areas where the majority of elephant in the group were seen standing motionless, apart from ear and tail movement, or lying down and not feeding. Foraging sites, the focus of this study, were areas where the majority of the herd were seen collecting and ingesting food at the time of observation. Once the activity of the group was categorised we then counted the number of elephant in the group and then waited and allowed the group to move before sampling the site. These observations were carried out on the two separate groups.

\section{Site characteristics}

Foraging sites refer to areas in which we observed the herds during April-May 2011. These were the sites where recursion was measured over 12 months. The habitat type of each site was categorised as one of open grass areas along forest margins, lowland dipterocarp forest, semi-inundated dipterocarp forest, swamp and recently logged or cultivated land according to previous classifications (Azmi, 1998; English unpub. data, Chapter Three). Distance to the nearest water source $(\mathrm{m})$, proximity to human habitation $(\mathrm{m})$, shade $(\%)$ and understorey cover (\%) were also recorded at each site. Distance to water and proximity to human habitation were measured using a Garmin 60csx Global Positioning System (GPS). Water 
sources included rivers, tributaries and ox-bow lakes but did not include swamps of stagnant, non-free flowing water. Proximity to human habitation was the distance in metres to areas with frequent human presence such as villages, dirt roads, bitumen roads and plantations. Shade percentage was measured using a spherical densiometer. This tool enabled calculation of the percentage of the forest canopy cover which was reflected onto a 24 cell grid convex mirror and measured near the centre of where the herd had been observed (see Lemmon, 1956). Understorey cover was measured using a $2 \mathrm{~m}$ long PVC pipe marked every $2 \mathrm{~cm}$ along its length. The number of $2 \mathrm{~cm}$ marks covered by vegetation was counted from a distance of $5 \mathrm{~m}$ when the pipe was held horizontally $30 \mathrm{~cm}$ from the ground by an assistant. This was then converted to a percentage of understorey cover in an area resembling the understorey vegetation before elephant disturbance.

\section{Defining and measuring site recursion}

A site was defined as the area that covered $100 \mathrm{~m}$ radius surrounding each measurement point taken from the centre of the elephant herd (Appendix One). The density of hourly GPS recordings was used as a measure of the amount of time spent within a $100 \mathrm{~m}$ diameter of the measurement point at each foraging site and was quantified for comparison between recursion and non-recursion foraging sites. The number of individuals in each group was counted on one hundred separate occasions for each group and was between 20-30 individuals per group (Group $1=25 \pm .24$ and Group $2=26 \pm .26$ ).

Using the recorded GPS positions from the elephant collars uploaded to Google Earth (Google Inc. 2010) along with the locations of foraging sites, we counted the number of returns to each site within 12 months, since the date of direct observation. The time period between each return was quantified. GPS points with a Positional Dilution of Precision (PDOP) of $>6$ were removed from analyses due to lack of precision (Langley, 1999).

\section{Statistical analyses}

The data collected from both elephant groups, was analysed using a t-test to compare differences in the frequency of recursion events, density of points at sites and the above mentioned habitat variables. The only variable that was significantly different between the 
two elephant groups was shade\% (Appendix Two). However this variable was later removed from further analyses and so the datasets were pooled (see below).

A Principal Components Analysis (PCA) reduced confounding effects of partial correlation between environmental variables (distance to water, proximity to human habitation, shade $\%$ and understorey $\%$ ). The PC identified that understorey and shade were strongly associated with Eigenvalues $<1$ and were therefore removed from further analyses, thus reducing the number of covariates in the model to distance to water and proximity to ongoing human habitation.

A Generalised Linear Model (GLM) (SPSS version 18.0, 2009) was used to determine if foraging site characteristics including distance to water, proximity to human habitation and habitat type influenced the dependent variable of recursion $(1=$ recursion $0=$ no recursion $)$. The amount of time the herd spent at a foraging site at the first visit (when the herd was initially observed at the site) was also included to determine if this influenced whether the site was later returned to.

A further GLM was used to analyse foraging sites with recursion to determine if there was a relationship between the time spent at the site at the first visit, time (days) between visits, proximity to human habitation, distance to water, habitat type and the dependent variable - time spent at the site at recursion.

The number of foraging sites where elephants were seen feeding on grasses/bamboo, gingers, palms, lianas and woody species (Table 1) was quantified and compared between recursion and non-recursion sites using a one-way ANOVA test . Food plants selected were measured from direct observation of elephants feeding, with plants seen ingested by the elephants identified to species level and later categorised into the above growth forms.

A chi-square test was used to determine if recursion frequency varied over temporal scales or if variation occurred randomly with no significant influence of time. 
Table 1. Plant species and their growth form selected by elephants at foraging sites in the Lower Kinabatangan Wildlife Sanctuary, Sabah.

\begin{tabular}{lll}
\hline Family & Genus & Plant form \\
\hline Poaceae & Dinochloa scabrida & Grass/Bamboo \\
Poaceae & Pannicum sp. & Grass \\
Poaceae & Phragmites karka & Grass \\
Poaceae & Pennisetum sp. & Grass \\
Zingiberaceae & Alpinia ligulata & Ginger \\
Maranthaceae & Donax canniformis & Ginger \\
Arecaceae & Licuala sp. & Palm \\
Arecaceae & Calamus caesius & Palm \\
Arecaceae & Arenga sp. & Palm \\
Leguminosae & Spatholobus sp. & Liana \\
Leguminosae & Fordia splendidissima & Liana \\
Lophopyxidaceae & Lopophaxis mangai & Liana \\
Dilleneaceae & Dillenia excelsa & Woody species \\
Sterculiaceae & Pterospermum sp. & Woody species \\
Euphorbiaceae & Mollotus sp. & Woody species \\
Euphorbiaceae & Claoxylon sp. & Woody species \\
Euphorbiaceae & Maccaranga sp. & Woody species \\
Myrtaceae & Eugenia sp. & Woody species \\
Moraceae & Ficus sp. & Woody species \\
Guttiferae & Garcinia parvifolia & Woody species \\
\hline & &
\end{tabular}

\section{Results}

\section{Recursion}

Eighty-seven foraging sites were measured. Seventy-five movement and 43 resting sites were also initially measured but not included in the analyses for this study, which is focusing on recursion to foraging sites only. Foraging sites were returned to by each herd over varying temporal scales ranging from within days, to weeks and months of the previous visit. Fortyeight foraging sites were returned to within 12 months. A large percentage of sites were returned to within 48 hours of the previous visit and the remaining sites were returned to over varying time-scales peaking again between 151-250 days. The difference in recursion 
frequency through time, therefore, was not random (Chi-square, $\mathrm{df}=10, \mathrm{P}=0.00$. Figure 2). Elephants spent more time at the first visit compared to the second visit if they returned within 48 hours to a site (t-test, $\mathrm{N}=11, P=0.00$ ).

We found a significant positive relationship between the hours at a site at the first visit and recursion $\left(x^{2}{ }_{1}=10.68, P=0.001\right.$, Figure 3$)$, with more time spent at sites that were returned to. No relationship was found between hours at the site on first visit and other biological or environmental site characteristics including distance to water, proximity to human habitation or habitat type.

We also found a significant positive relationship between the number of days between visits (Figure 4a), time spent at a site at the first visit (Figure 4b) and habitat type (Figure 4c) with the amount of time at a site at recursion (hours at first visit, Wald chi-square $=89.51$, $\mathrm{df}=1, P=0.00$, days between returns, Wald chi-square $=13.77, \mathrm{df}=1, P=0.00$, habitat type, Wald chi-square $=3.44, \mathrm{df}=1, P=0.043$ ).

From direct observations of the elephant herds foraging we found that there was a significant relationship between recursion and foraging sites where they were seen feeding on grasses and bamboos (ANOVA, $\mathrm{df}=86, \mathrm{~F}_{1,86}=14.041, P=0.000$ ) and a significant relationship between feeding on lianas and foraging sites that were not returned to within the 12 month study period (ANOVA df $=86, \mathrm{~F}_{1,86}=8.501, P=0.005$ ) (Figure 5). Woody plants, palms and gingers had no significant influence on recursion $(P=0.095, P=0.157$ and $P=0.504$ respectively). 


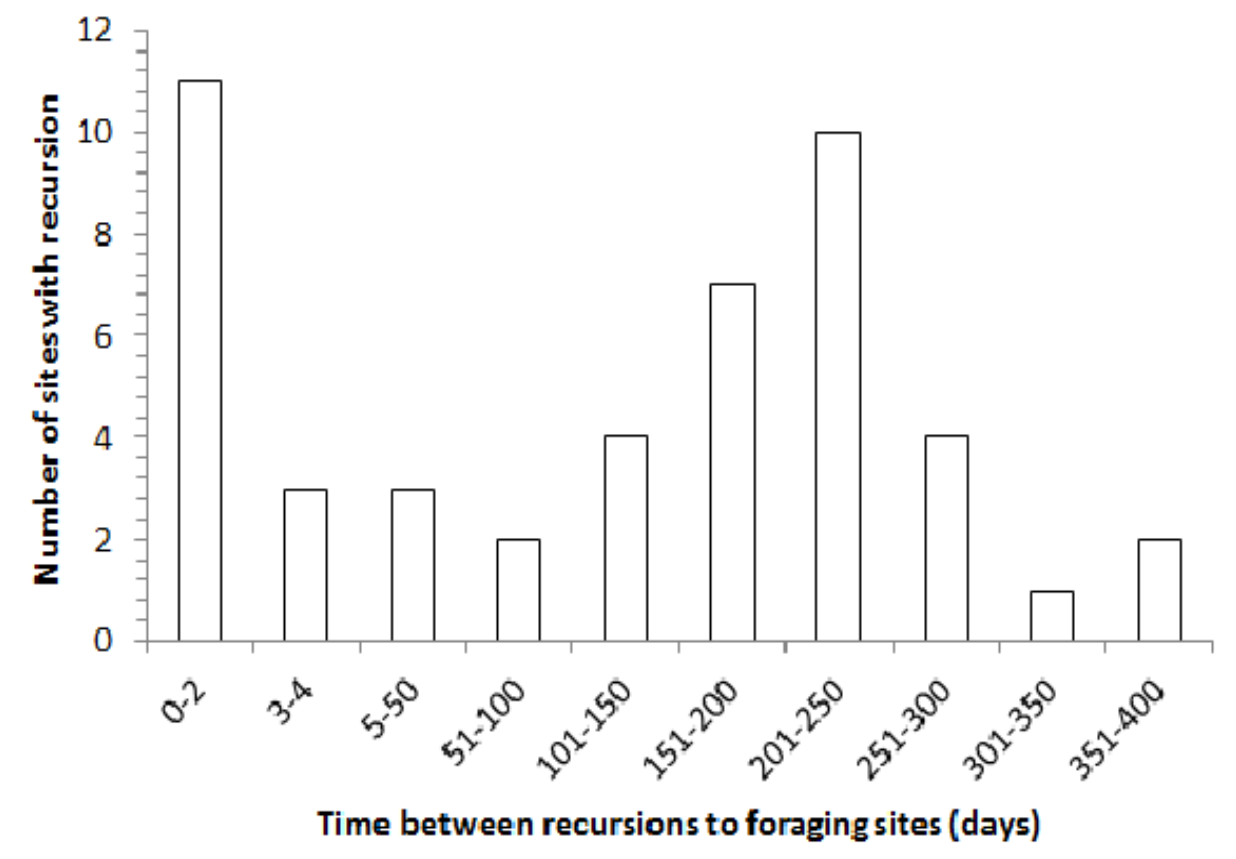

Figure 2. Time period (days) between recursions by Bornean elephants in the Lower Kinabatangan Wildlife Sanctuary, Sabah, Malaysia over a 12 month period. A total of 48 foraging sites out of 87 were returned to.

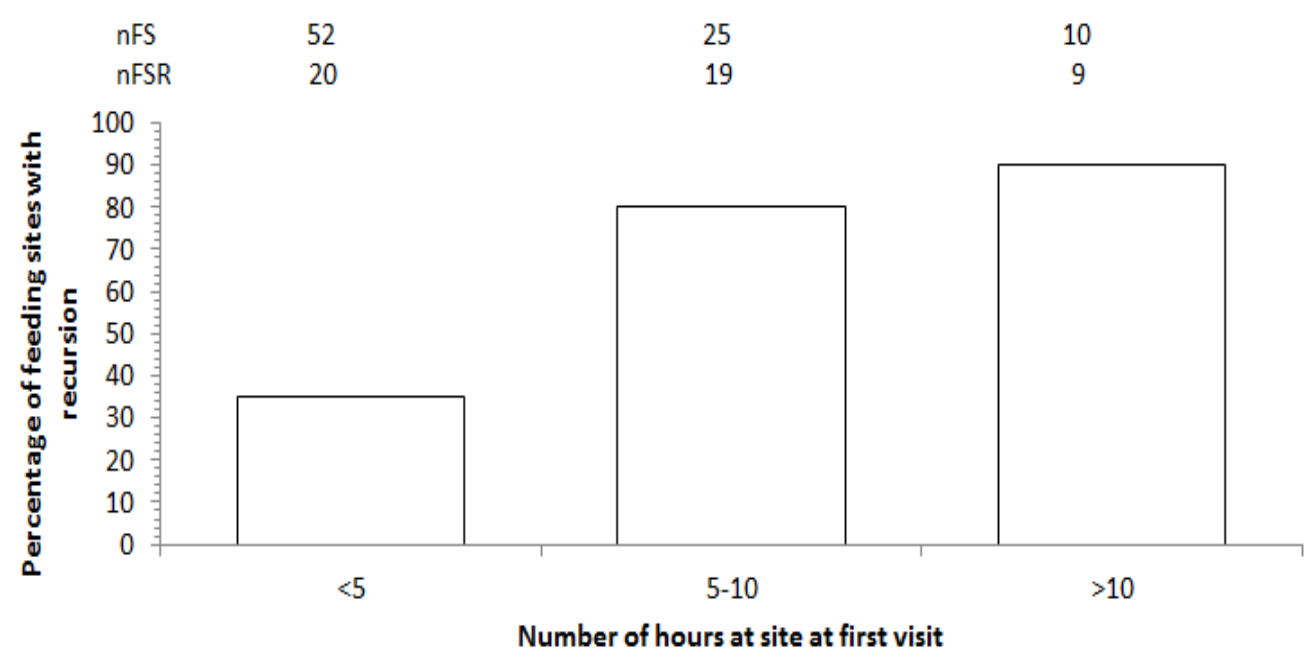

Figure 3. Comparison between the time spent (hours) at recursion and non-recursion sites within the Lower Kinabatangan Wildlife Sanctuary, Sabah. The occurrence of recursion was higher to foraging sites where elephants had spent more time at the previous visit. nFS is the total number of feeding sites $=87, \mathrm{nFSR}$ is the total feeding sites with recursion $=48$. 


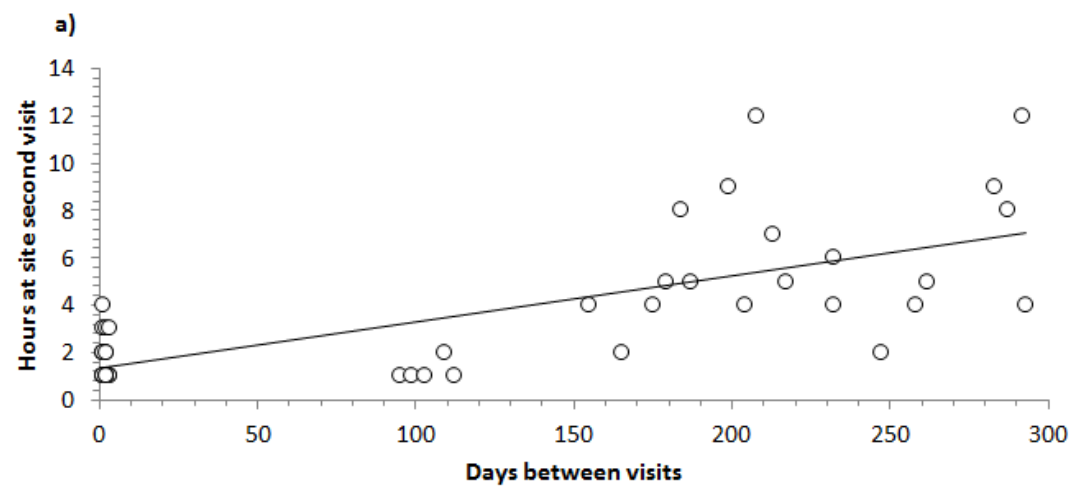

b)

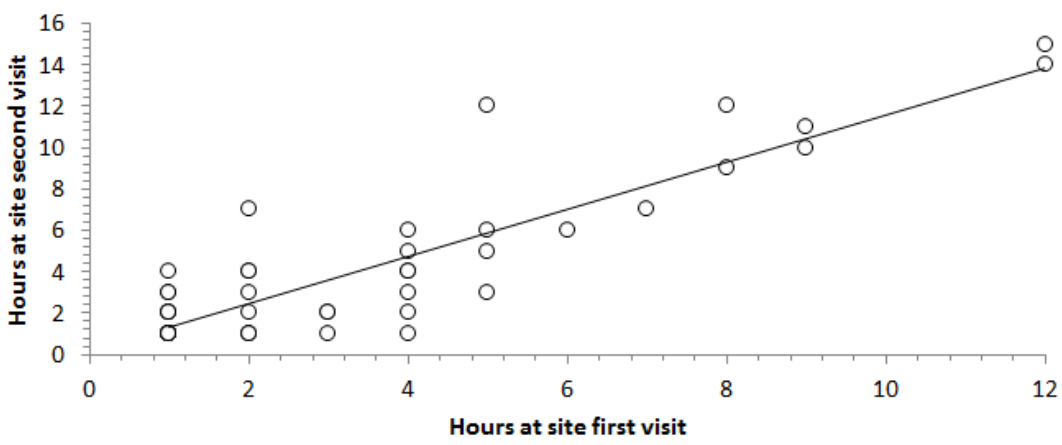

c)

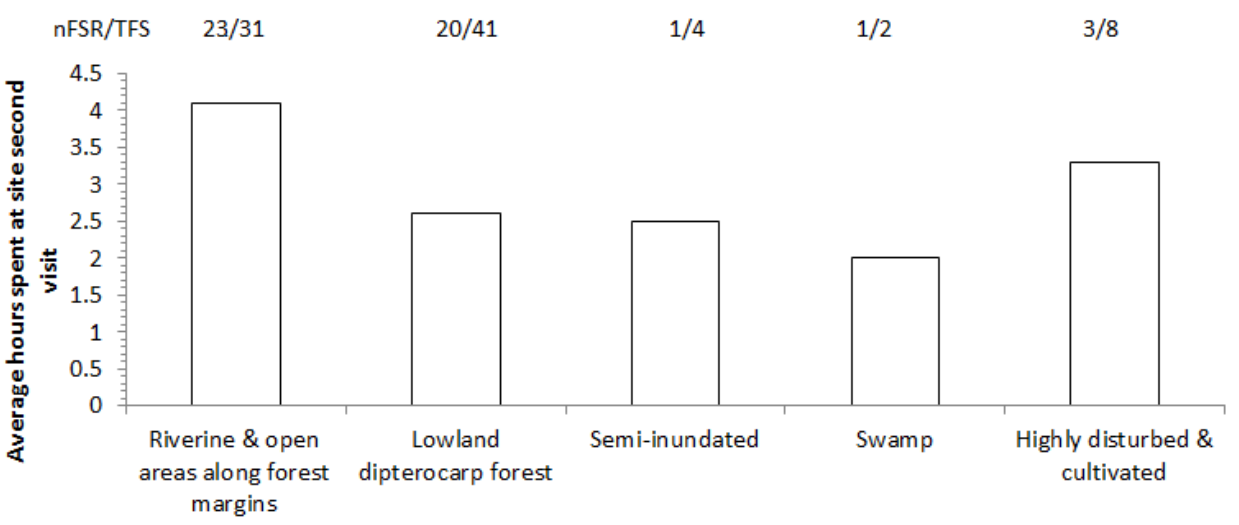

Habitat type

Figure 4. Variables influencing time Bornean elephants within the Lower Kinabatangan Wildlife Sanctuary, Sabah spent at second visit to a site (i.e. $1^{\text {st }}$ recursion) a) days between visits b) time spent at sites at first visit and c) habitat. nFRS is the number of foraging sites that received recursion and nTFS refers to the total number of foraging sites within each habitat type. 


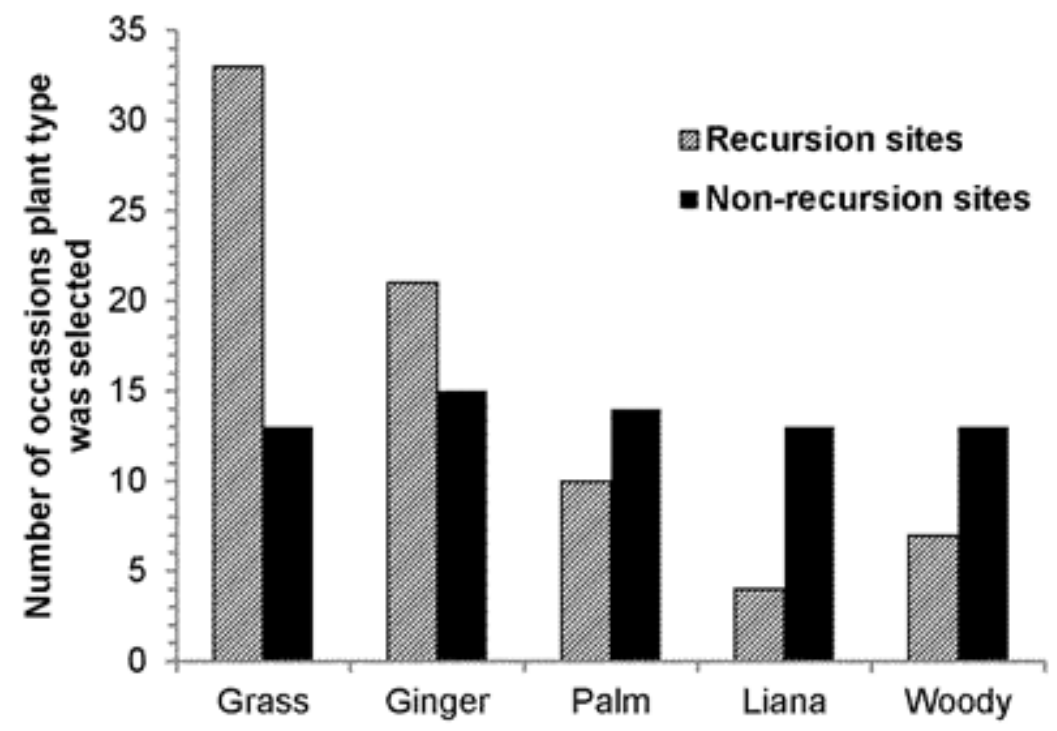

Figure 5. Plant types selected for feeding by Bornean elephants within LKWS at recursion and non-recursion sites.

\section{Discussion}

Recursion by elephants in the LKWS was common. It most commonly occurred during two time periods: within 48 hours of the previous visit and between 151-250 days after the last visit, suggesting there are two different reasons for recursion.

We also found that the occurrence of recursion to foraging sites was influenced by the amount of time elephants had spent at a site previously, with recursions occurring more often to sites where they had spent more time in the past. A positive relationship was found between the number of days between the first visit and recursion, the number of hours spent at a site at the first visit, and the amount of time spent at a site at recursion. The habitat type of the site also influenced how much time was spent at a site during recursion. Additionally, elephants fed more on grasses and bamboos at recursion sites compared to sites they did not return to. Grasses and bamboos are most commonly found in the open grass areas along forest margins (English et al., 2014c) where elephants spent more time at recursion. 
A relationship between the amount of time allocated to a particular area per visit and the resource quality of the area has previously been identified in large herbivore foraging behaviour (Bailey et al., 1996). Our results suggest that, as more time was spent at recursion sites compared to non-recursion sites, then these sites, and sites with similar characteristics as recursion sites, are favourable to elephants and therefore should be considered high quality.

\section{Short -term recursions}

Recursions to a foraging site within 48 hours of the previous visit were expected to be a sitesampling strategy where short duration visits were followed by longer duration visits, with the elephants returning to feed more extensively once the site quality was known relative to other sites in the area. We found the opposite, however. Elephants spent more time at sites at the first visit compared to the second visit (recursion) when they returned within 48 hours. This foraging strategy may be a way of reinforcing acceptance of a site over time (OwenSmith, 2002). Alternatively, shorter second visits might be due to the elephants checking that all food within the site was exploited, resulting in less feeding time due to reduced food quality and insufficient regrowth of vegetation. Furthermore, induced responses in plants following herbivory such as increased tannin content (Karban \& Myers, 1989), could reduce the palatability of plants at sites recently visited.

\section{Long-term recursions}

Recursions after longer periods may be related to plant recovery rates (Bar-David et al., 2009) where herbivores return to exploit a feeding site once resources have sufficiently recovered. The more time elephants spent at sites at the first visit, the more days passed before they returned and the more time was spent at the site at recursion. This suggests that recursion occurring after 151-250 days is most likely related to resource recovery. To minimise energy expenditure and make optimal use of its home-range, a herbivore would benefit by monitoring vegetation depletion and renewal rates (McNaughton et al., 1997). This strategy would reduce the occurrence of returning to areas that had recently been exploited and facilitate the return after resource renewal.

Elephants may shift among sites in the same general region or move between a set of foraging areas. Recursions that occur after a longer time-period may occur after all sections have been exploited and enough time has passed for vegetation to regenerate in the areas previously visited (Bailey et al., 1996). This differs from recursions that occur within 48 
hours where insufficient time has passed for resource regeneration. Long-term recursions to previously foraged areas, therefore, may be a beneficial foraging strategy for herbivores, enabling them to consume regrowing vegetation in a stage of high primary productivity and palatability (McNaughton, 1985; Gordon \& Lindsay, 1990). Moreover, recursions may accelerate nutrient cycling in highly foraged sites (Gordon \& Lindsay, 1990; McNaughton et $a l, 1997$ ) and maintain them as nutrient hotspots (Winnie et al., 2008). Elephants may also select certain plants at a foraging site at specific times of the year. This could explain why some sites received less recursions than others, for example where they had selected woody plants previously.

Both long- and short-term foraging strategies may be a form of associative learning, although in some species, returning to a resource once it has been replenished is innate (Burke \& Fulham, 2003). Innate foraging decisions associated with the spatial and temporal availability of resources may also occur in elephants. However, for such a highly social and long-lived species with large home-ranges, learned behaviour within elephant herds is likely, especially considering their highly developed spatial and temporal memory (Hart et al., 2008). It is therefore probable that elephants remember areas containing their preferred food choices and return to them after sufficient time has passed for resources to replenish.

\section{Implications for elephant habitat management}

The identification and conservation of sites of recursion for elephants should be a priority in the design and management of reserves. For the LKWS population, open grass areas along forest margins appear to be key recursion sites and so these areas should be a conservation priority. Loss of access to recursion sites, or sites with similar characteristics, from either anthropogenic or natural disturbance may lead to increased human-elephant conflict due to resources being sought elsewhere, such as in oil palm plantations. Additionally, conservation practitioners and wildlife departments should establish protected corridors linking recursion sites to ensure accessibility of these for elephants.

The temporal pattern of site recursion described in this study is a reflection of elephant movement patterns amongst foraging sites. An investigation of movement was beyond the scope of the present study but further studies of recursion will benefit from investigations of the movement patterns that support recursion over different temporal scales. We observed recursion to occur over two time-scales: short- and long-term. Such a recursion could be supported by random or stratified random movement patterns, especially given 
spatial constraints of habitat boundaries, and so potentially modelled using random-walk models: e.g., correlated random walks (McCulloch \& Cain, 1989). Alternatively and much more likely, long-term recursions occur as a result of lengthy, directed moves to clusters of foraging sites followed by short-term recursions within the clusters. This type of movement would be expected to follow a Hidden Markovian Model (Langrock et al., 2012) (Appendix Three). The models that best explain movement pattern while also generating observed patterns of recursion remain to be found.

\section{Acknowledgements}

Thank you to Victoria University of Wellington for supporting the study and providing a doctoral scholarship and a completion scholarship for Megan English and to the Economic Planning Unit and Sabah Wildlife Department for permitting the research in the Lower Kinabatangan Wildlife Sanctuary. Thank you to Sulaiman Ismail from HUTAN Elephant Conservation Unit for helping with data collection and Danau Girang Field Centre and Nurzhafarina Othman for allowing access to the collared elephant data. Thank you to Andrew Hearn from the Bornean Clouded Leopard Programme for use of the map of LKWS. This project was supported by funds to Megan English and Dr Wayne Linklater from Zoos Victoria, Australia. 


\section{Chapter Five}

Recursion to browsed plants by the Bornean elephant

Authors note: An updated version of Chapter Five was published in the journal PeerJ in August, 2015. 


\begin{abstract}
Plant recovery rates after herbivory are thought to be a key factor driving recursion by herbivores to sites and plants to optimise resource-use. I investigated the relationship between plant recovery and recursion by elephants (Elephas maximus borneensis) in the Lower Kinabatangan Wildlife Sanctuary, Sabah. The recovery growth of 156 recently eaten food plants, from 30 species, was measured along 14 transects over 9 months, or until the plant was re-browsed by elephants. The average monthly growth in length was used to calculate the time required for each plant and species to recover to its previous length. Recursion occurred to 12 of the 30 species, which was $58 \%$ of all plant samples previously browsed. Moreover, $77 \%$ of these re-browsed plants were in the Poaceae family. Recovery times varied from 2 to more than 12 months depending on the species. Recursion to all plants in the Poaceae coincided with plant recovery whereas recursion to most browsed plants occurred before they had recovered to their previous branch or shoot length. The small sample size of many browse plants that received recursion and the uneven plant species distribution across the transects limited the analyses in this study. Nevertheless a prominent pattern in plant scale recursion did emerge. Species and plant recovery time most strongly influenced the time to recursion. A relationship between the recursion time and the plant recovery time of the elephant's preferred food, grasses, was evident. Elephants are bulk feeders so it is likely that they time their returns to bulk feed on these grass species when quantities have recovered sufficiently to meet their intake requirements but this was not true of browsed species. The implications for habitat and elephant management are discussed.
\end{abstract}




\section{Introduction}

Wild herbivores repeatedly utilise areas within their home-range. Recursion, the repeated use of the same sites within a home-range or fine-scale reuse of resources (e.g. plants by herbivores) within a site over time is thought to be an optimal-foraging strategy. Few studies have measured wild herbivore recursion. Nevertheless, the few that have investigated recursion found it to occur at site and landscape scales (Bar-David et al., 2009; Benhamou \& Riotte-Lambert, 2012; Riotte-Lambert et al., 2013). Although finer-scale studies of wild large herbivore recursion do not exist, it has been suggested that recursion to foraging sites may be influenced by plant recovery rates and reuse (Bar-David et al., 2009). Studies of recursion, and the growth and recovery of plants after herbivory, may have significance for understanding habitat-use and food preference, providing a greater understanding of herbivore foraging strategies. Prior to this study, individual plant growth rates and their influence on recursion had not been explored systematically on wild herbivores (Chapter Two).

Recursion to previously browsed or grazed sites and plants may be an optimal foraging strategy enabling consumption of re-growing vegetation in stages of highest productivity (McNaughton, 1985; Gordon \& Lindsay, 1990). Recursion to plants after nectar has replenished occurs by insects and birds (Davies \& Houston, 1981; Bell, 1990; Williams \& Thomson, 1998) Williams \& Thomson, 1998; Davies \& Houston, 1981) and recursions to trees for fruit occurs by primates (Garber, 1988; Garber \& Jelinik, 2006; Erhart \& Overdorff, 2008; Janmaat et al., 2013; Porter \& Garber, 2013). But this remains to be explored in wild large herbivores. Herbivore recursion may accelerate nutrient cycling in highly grazed sites (Gordon \& Lindsay, 1990, McNaughton et al., 1997) and so maintain them as nutrient hotspots (Winnie et al., 2008). Thus, recursion may trigger and maintain positive feedback between large herbivore movement and feeding, and vegetation regeneration and palatability (McNaughton et al., 1997). Plant recovery period may, therefore, be a strong influence on recursion frequency (rate) and herbivore movement.

Studies of recursion have implications for animal population and habitat management. Most evaluations of wild animal resource requirements and preferences are based largely on the premise that if animals visit or feed on resources (e.g. site, plant or plant species) in lower or higher proportion to their availability then this suggests that the resource is avoided or preferred, respectively (Johnson, 1980). This framework is most commonly applied in studies 
that occur over relatively short time-frames to provide an indicative 'snap-shot' of resourceuse. However, in natural environments resources are not consistently available in distribution, proportion and density through time and animals may reuse some resources but not others. A food species, for example, may appear to be a minor or trivial part of the diet at selected sites but might be the subject of repeated use such that recursion would illustrate it to be highly selected. Alternatively, less common foods may appear to be avoided until investigation of recursion reveals reuse. Thus, studies of recursion are necessary to elaborate on spatial variation in availability and selection when assessing food and habitat preferences.

Recursion patterns may also be a useful indicator of population relations with habitat. Large herbivores, like elephants, are a particularly interesting species in which to study recursion as they are ecosystem engineers, having complex, scale-dependent effects on habitat structure and vegetative community (Bond, 1993; Jones et al., 1996). A study of recursion at the plant scale can identify if elephants are potentially depleting resources by rebrowsing before plants have recovered, or if they are facilitating growth of preferred or bulk food plants. If recursion is too frequent, selected plant species might be over-utilised. Thus, increases in rates of recursion that exceed plant recovery rates could indicate that a population might exceed habitat capacity or that the herbivore has a major influence on vegetation community structure and composition. Alternatively, recovery rates that exceed recursion may be evidence of further capacity to support greater herbivore densities. An understanding of recursion patterns, therefore, may augment habitat condition evaluation in herbivore population management, reserve design, and understanding the landscape's capacity to support elephants.

Previously I have shown variation in recursion due to site characteristics (Chapter Four). I now aimed to test if recovery time contributes to explaining species and site recursion patterns by testing the hypothesis that recursion to plants by elephants in tropical rainforest is influenced by plant recovery rates. I predicted that elephant recursion would coincide with plant recovery.

\section{Method}

\section{Study site and focal species}

See general Introduction, pages 26-28. 


\section{Plant recursion}

Fourteen $50 \mathrm{~m}$ transects were established where elephants had fed previously. Transects were $>300 \mathrm{~m}$ apart (See Chapter Six, Figure 1). One transect was established per day. We tracked fresh elephant signs including footprints, dung and signs of feeding to establish the transect along the group's feeding path. All plants showing signs of elephant feeding within $2 \mathrm{~m}$ either side of transect were marked and labelled with the date and a reference number. Samples of all plant species were collected for identification at the Sabah Forestry Department Herbarium (SAN), Sandakan. The growth and recovery of each plant after herbivory was measured each month from April 2011 to December 2011 or until the elephants re-browsed the plant's new growth. The length of the plant stem prior to browsing or grazing was determined by measuring the length of stems of the same plant that were not eaten, or remnants of the eaten stem, as a surrogate reference of original stem length. If the plant died or the new growth was re-browsed by other herbivores, thus preventing measurements of regrowth, this was recorded. It was possible to differentiate between elephant feed sign and other herbivore feed sign, such as from wild boar (Sus scrofa) and Sambar Deer (Rusa unicolor), because of the other sign and spoor left in the area, such as dung, footprints, the way in which the plant was eaten and the height of the sign. Recorded GPS positions of the two collared elephants from the two main herds in LKWS (See Chapter Four, Methods) confirmed when the focal group returned to the site and transect.

Plant physiognomy varies between species and between plants within a species. Regrowth measurements were taken on a selected new shoot closest to the growth node nearest the feed sign, or from the plant base, depending on plant physiognomy and how it recovered (see Figure 1 for typological examples). Measurements included new shoot growth in length and basal diameter, and a count of the number of new shoots produced each month. The approximate length and the basal diameter of the original feed sign on each plant were compared to the length and basal diameter of the new growth when it was fed on again. If we returned to a plant and it had been fed on since the last measurement was taken, the growth measurements from the prior month were used for comparison. The same technique was used for the two Poaceae species Phragmites karka - a reed, and Dinochloa scabrida - a bamboo, 
as their structure is a main stem with new growth emerging from nodes along the main stem, or from the root system. Short grasses were not included due to difficulty in identifying feed sign (i.e. the whole plant is often ingested) and measuring recovery growth related to feeding by elephants.

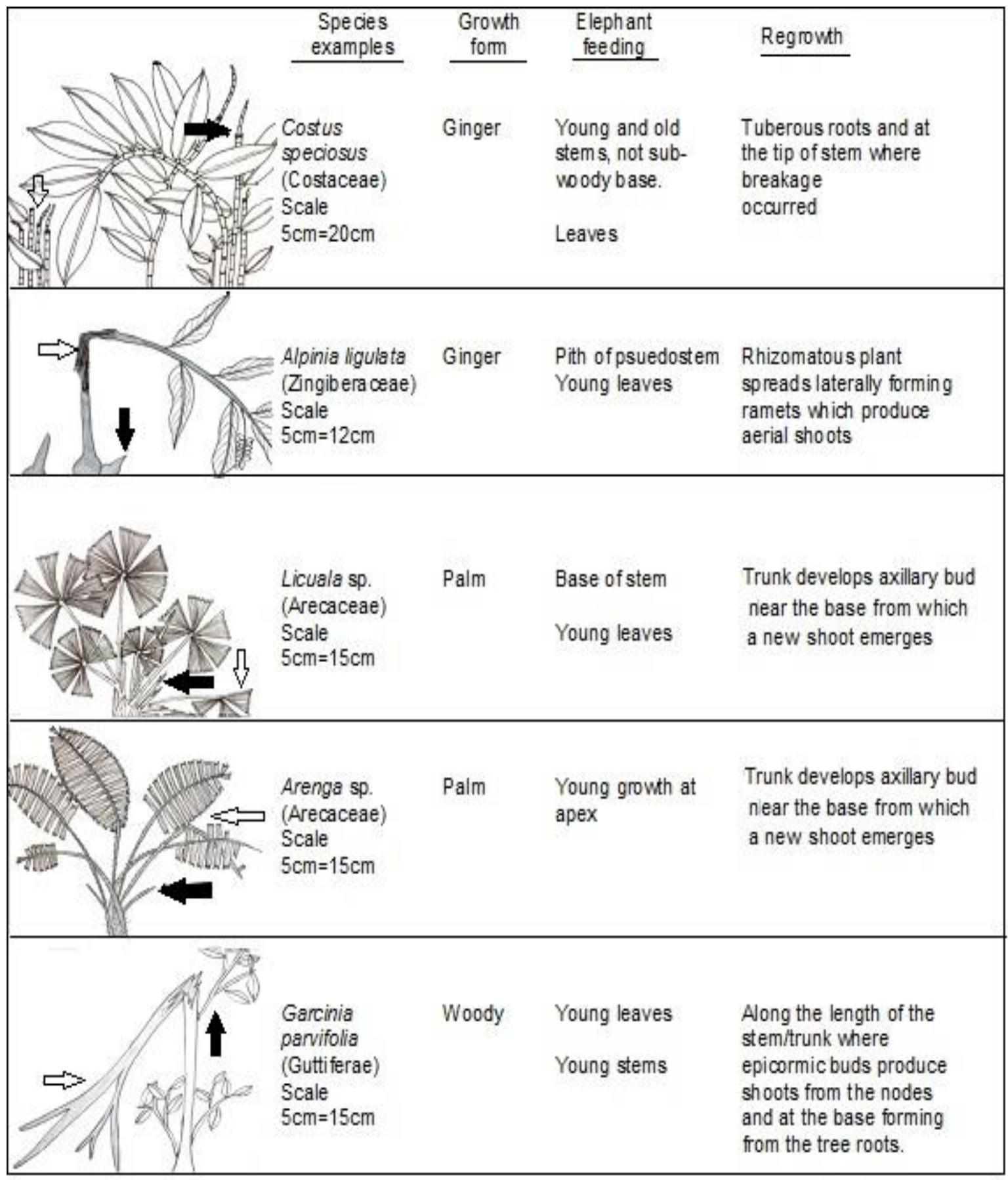

Figure 1. Examples of plants selected by elephants in LKWS showing plant growth forms and their recovery. White arrows indicate portions of the plant eaten by elephant and black arrows indicate recovery growth. 


\section{Plant recovery growth and recursion rate}

The expected time required for each plant to recover was estimated by averaging the monthly growth in length $(\mathrm{mm})$ of the plant, divided by its estimated length at the beginning of study which was based on what remained of the stem after elephant feeding and other stems on the same plant as a surrogate reference. Based on this monthly growth average, I estimated how many months it would take for the plant to return to its previous length. Time until recovery and time to recursion were averages of multiple plants for each species.

\section{Multi-model inference and selection}

An information theoretic approach was applied to test the hypothesis for recursion time and plant recovery time. I predicted recursion would occur after plants had recovered to their preherbivory height. I evaluated the power of plant recovery to explain recursion in the absence of other a priori hypotheses by comparing a model of my hypothesis with models that included random effects for site (transect) and species. I described and evaluated models in the 'Ime4' package in R (Bates et al. 2014). All plants browsed by elephants, including those plants that did not receive recursion but were located on transects that received recursion, were included in the analyses. I used maximum likelihood (MLE) to provide estimates of the model's parameters because fixed effects were different between models.

\section{Results}

I recorded a total of 182 plants from 30 species eaten by elephants over 14 transects. Eightysix of these plants from 12 species were re-browsed, i.e. recursion to individual plants (Figure 2a). Twenty-six plants died and did not recover after being partially eaten by elephants and were, therefore, not included in further analyses (Figure $2 b$ ). Two transects were not returned to (5 plants each transect), resulting in 146 plants used for recursion analyses.

The time to recursion for each species varied across the nine months of sampling (Figures 3 \& 4). Four species including Costus speciousus, Dinochloa scabrida, Phragmites karka and Spatholobus sp., had recovered to their previous size when they were re-browsed, whereas the remaining eight species were re-browsed before they had fully recovered (Figure 
4). Seventy-seven percent of re-browsed plants were in the Poaceae family. A linear fixedeffects model found that the plant recovery time strongly influenced the time to recursion beyond influences that could be attributed to species differences and especially differences amongst sites (Table 2).

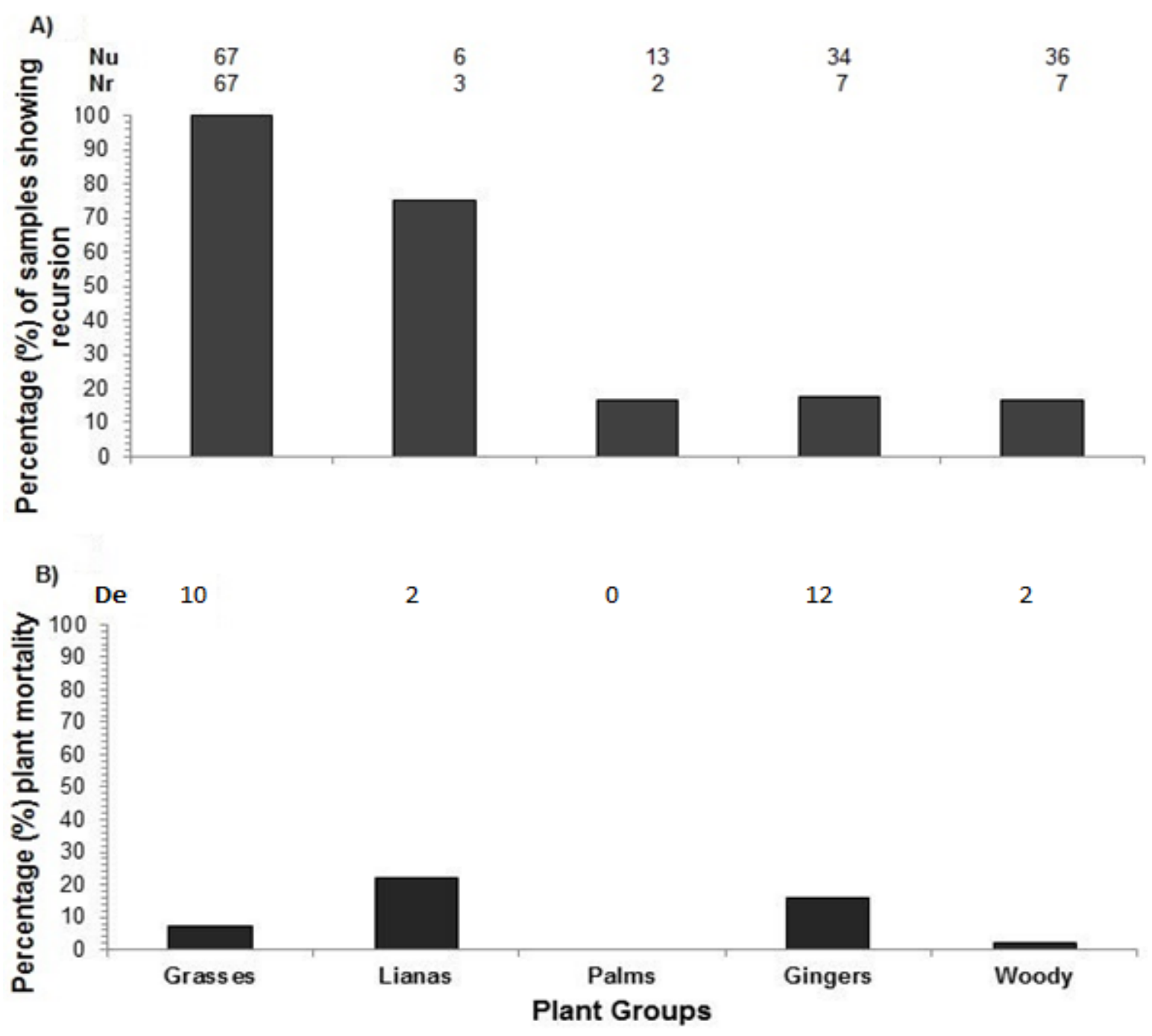

Figure 2. A) The percentage of plants along transects re-browsed by elephants. Nu number of plants of each plant group eaten by elephants at the first visit. $\mathbf{N r}$ number of plants of each plant group that were re-browsed B) Plant mortality within plant groups. De number of plants that died. 
Two species of Poaceae, Phragmites karka - a reed, and Dinochloa scabrida - a bamboo, received recursion at a time when their length was not significantly different from when they had first been selected (Reed t-test, $\mathrm{df}=50, P=.137$, Bamboo t-test, $\mathrm{df}=17, P=.232$ ) (Figure $3)$. However all other species that were selected were re-browsed before they had recovered to their previous length except one ginger species, Costus speciousus, and one liana species, Spatholobus sp. (Figure 4 A) i) and D) i)).

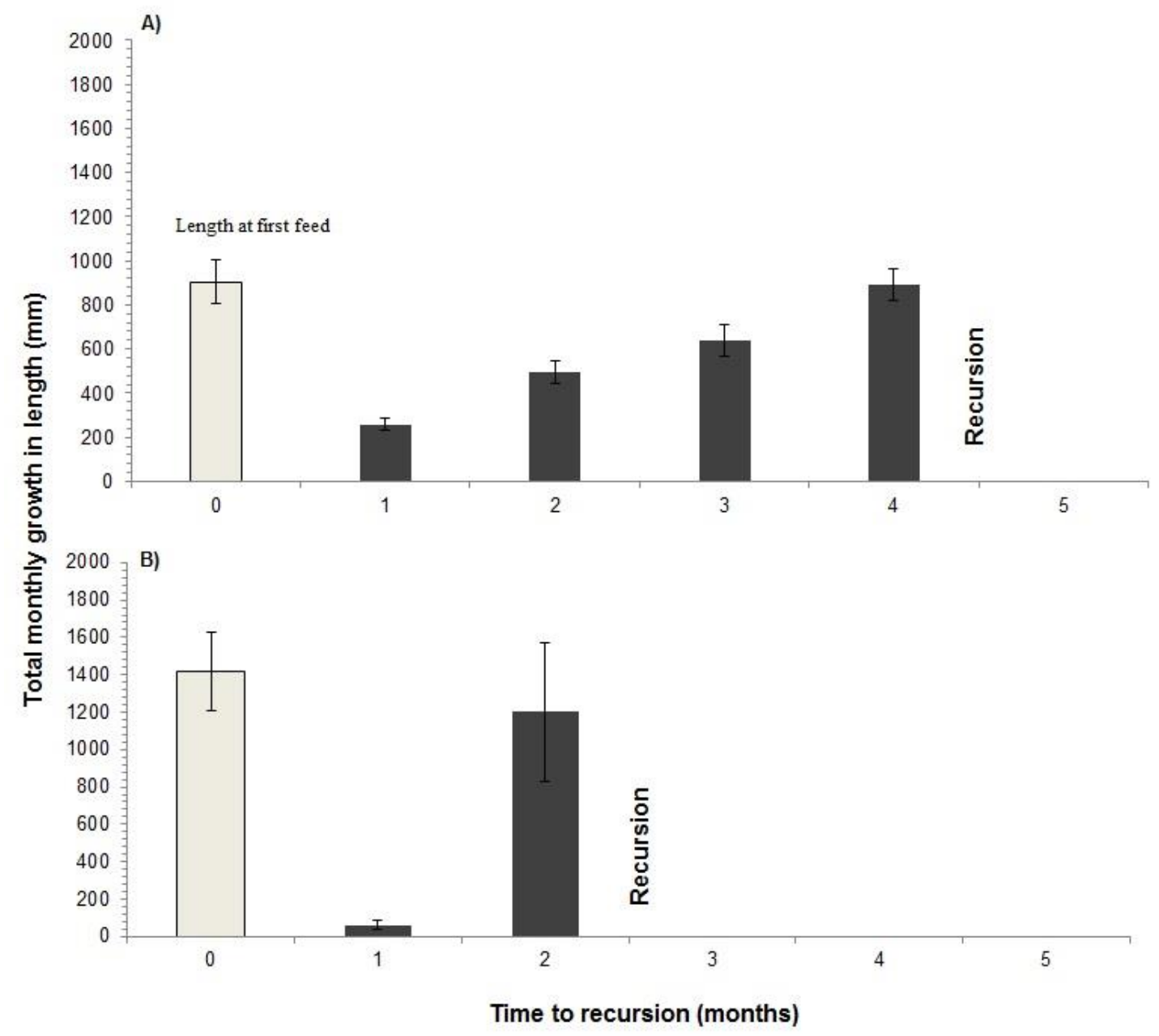

Figure 3. Recursion to grasses showing the average length of the grass stem when initially fed on (shaded bar) and the average total length of new shoots per month until recursion occurred, for two grass species A) Phragmites karka and B) Dinochloa scabrida. Standard error bars represent \pm 1 standard deviation of the sample distribution. Recovery has occurred when the black bar is the same length as the white bar. 


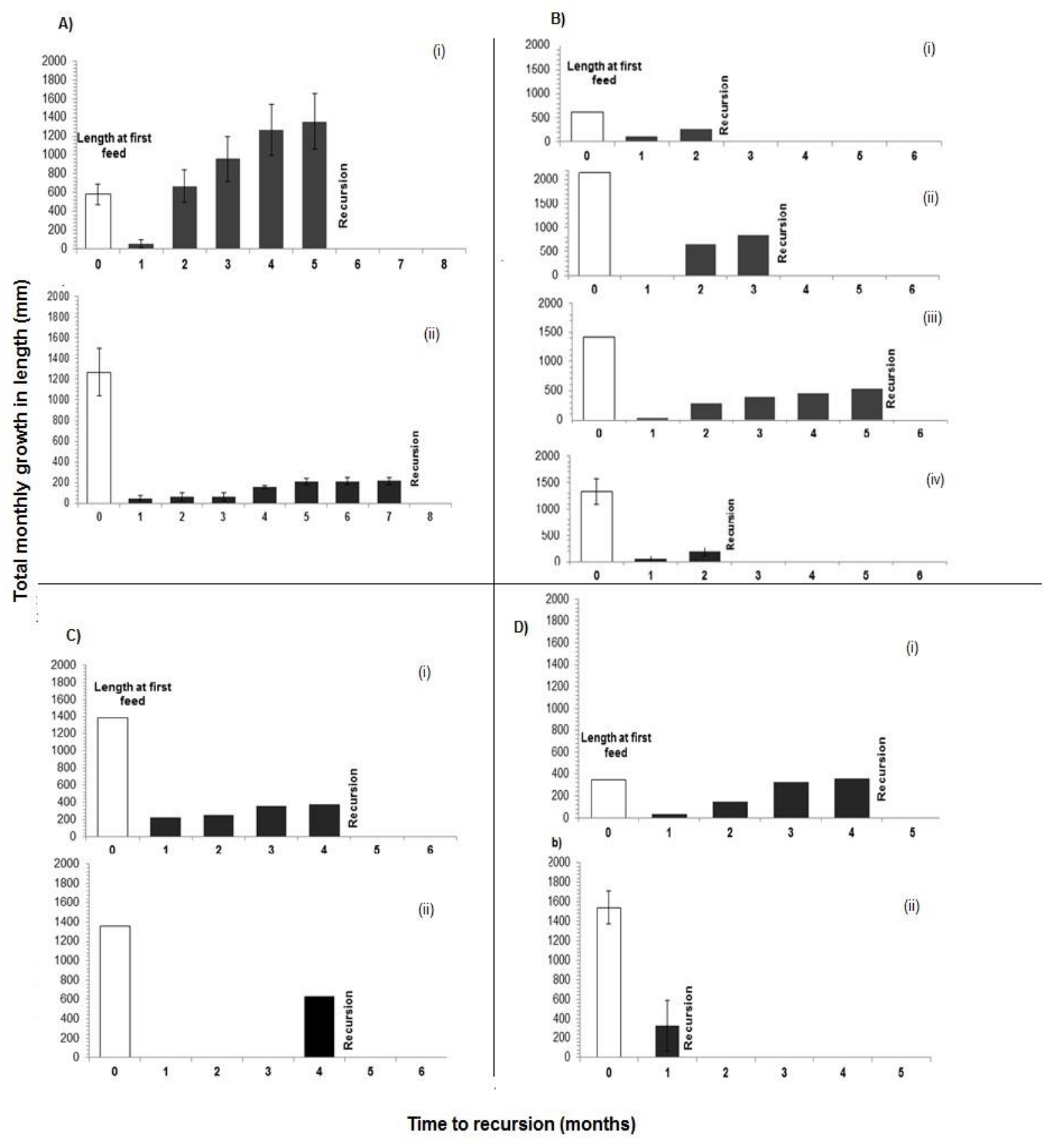

Figure 4. Recursion to gingers, woody species, palms and lianas. The average length of the stem when initially eaten (white bar) and average monthly total new shoot length of each species until re-browsing A) i) Costus speciousus and ii) Donax canniformis. B) i) Memecylon panniculum ii) Garcinia parvifolia iii) Claoxylon sp. and iv) Lepisanthes sp. C) i) Calamus caesius and ii) Arenga sp. D) i) Spatholobus sp. and ii) Fordia sp. Recovery has occurred when the black bar is the same length as the white bar. 


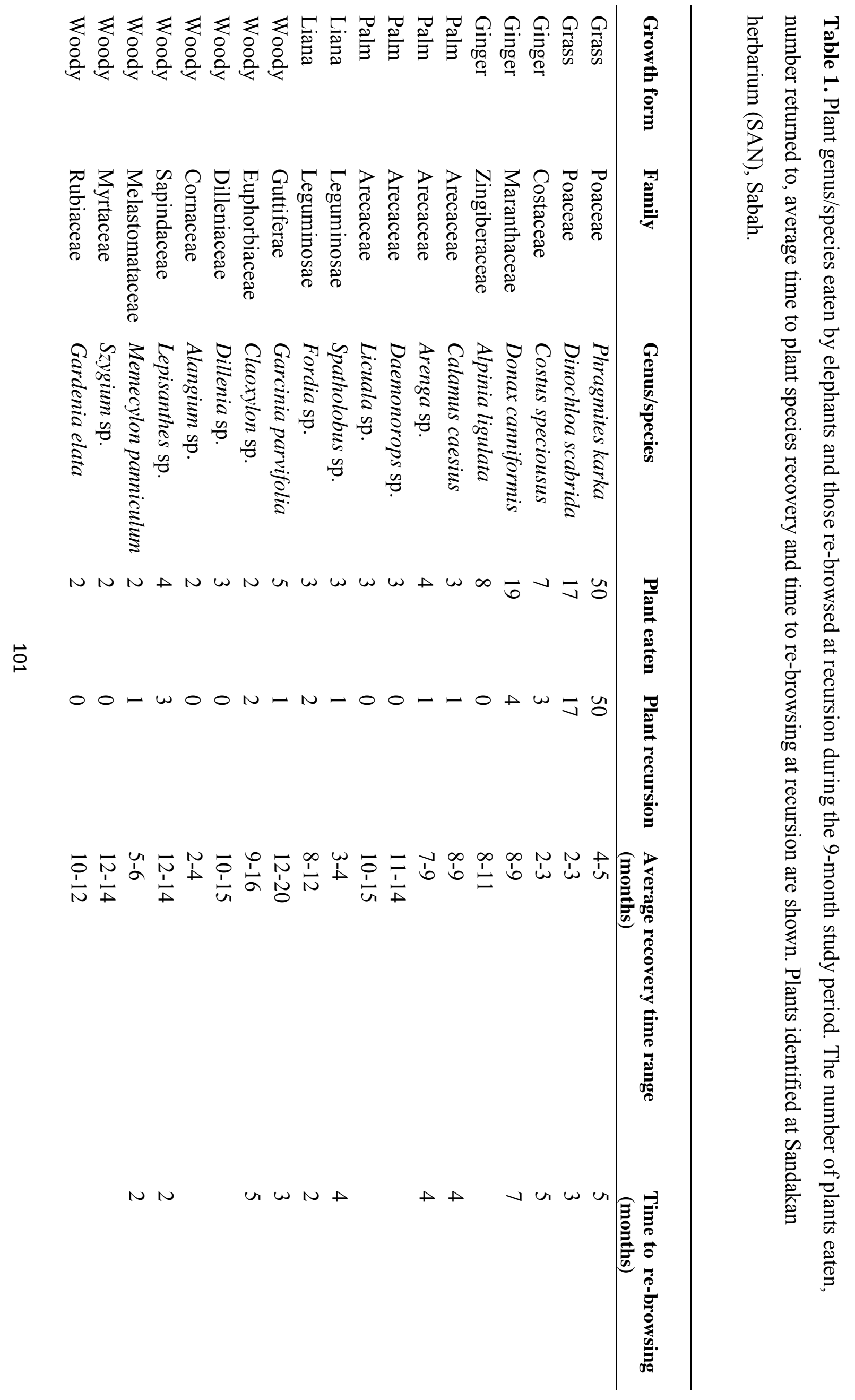




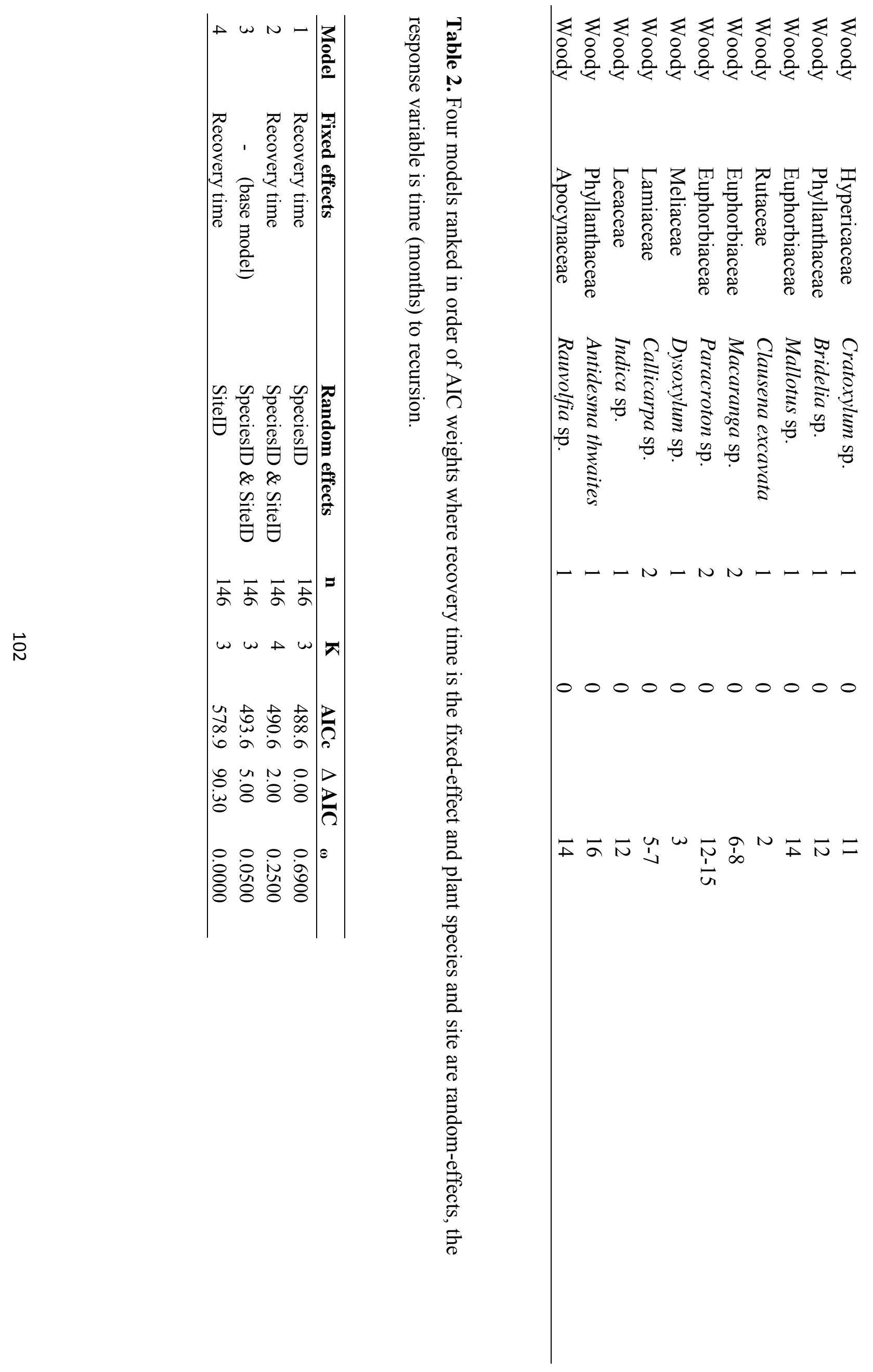




\section{Discussion}

Plant recovery time after herbivory as an explanation of site or plant recursion has not previously been explored in an uncontrolled environment with wild animals. However, it has been suggested that plant recovery influences the timing of returns to feeding sites (BarDavid et al., 2009). My results indicate that plant recovery time amongst a diversity of plants with varying physiognomy and recovery rates corresponds to recursion time to individual plants within the Poaceae. Recovery time, therefore, does appear to influence recursion but so do the characteristics of plant species. Recursion is also influenced at the site level (Chapter 4) but is not necessary to explain individual plant recursion time. We found that recursion rates were greatest for the species Dinochloa scabrida and Phragmites karka, in all cases these were returned to after they had recovered. These species have also been identified as preferred food plants of the elephants (Chapter Six, English et al., 2014c) and they made up $43 \%$ of all plants selected and $77 \%$ of plants receiving recursion. Elephants are bulk feeders so it is likely that they time their returns to bulk feed on grasses when stands have recovered sufficiently to meet their intake requirements. These grass species were less likely to die and fast to recover compared to other plants. Other than grasses, most species did not receive recursion, or if they did, it occurred before the plant had recovered.

Recursion to a few poorly regenerated species, such as woody trees, gingers and palms, may be a result of elephants foraging on other plants nearby and indiscriminately rebrowsing on unrecovered plants. If this was the case we would expect browse plants found within grass dominant sites to be inadvertently selected when the elephants re-browsed on the grass. Half of the browse plant samples found within grass dominant areas were re-browsed before recovery. Alternatively, recursion to unrecovered plants may be due to elephants specifically targeting younger growth where these plants form a small but important component of the diet. Plant productivity and nutritional quality in areas with dense herbaceous vegetation has also been found to be highest after around 5-6 months of plant regrowth (Plumptre, 1993), which might explain why unrecovered plants were re-browsed before recovery. Furthermore, elephants manipulate the structure and composition of their plant resources. For example, Jachman and Bell (1985) proposed that African elephants selectively fell preferred tree species to stimulate coppicing and increase the availability of palatable forage. Elephants may therefore alter structure and floristic composition, especially of woody species, in ways that increase rather than reduce carrying capacity, particularly if 
plant species respond positively to browsing by producing new growth. Furthermore, if woody plants do not respond positively to elephant feeding this might lead to the creation of open areas. These areas are likely to become dominated by early successional species, thus providing the elephants with more of their preferred food such as those from the Poaceae family. Therefore, feeding on woody species faster than they can recover may augment grass patches and prevent woody invasion. This is a common occurrence with elephants, as ecosystem engineers they are known to alter the structure and composition of habitat and plant communities (Laws, 1970; Bryant, 1981; Bergström \& Danell, 1987; du Toit et al., 1990; Ben-Shahar, 1993; Prins \& Olff, 1998). Elephant impact on woody vegetation has led to decreasing numbers of trees and increase of open areas in Africa (Conybeare, 2004; O'Connor et al., 2007). The results of my study suggest that elephants may be controlling reforestation within open grass areas by re-browsing on plants including woody species, lianas, gingers and palms before recovery, however a long-term study on elephants as ecosystem engineers within the Lower Kinabatangan is required.

Another plausible explanation for recursion on poorly regenerated plants is that these plants are highly desirable and resources in the area are inadequate (due to spatial constraints/population pressures) for the animals to have the option of lower frequency of recursion. Elephant feeding on plants before they have recovered might lead to certain species being over-exploited. Over-exploitation of preferred plant species may indicate that the elephant population is approaching or exceeding habitat carrying capacity. However, with the exception of grasses, our results show that only 19 of 89 browse plants were returned to for feeding during the 9-month study period. This finding suggests that there is no evidence from recursion data that this elephant population has exceeded the area's carrying capacity.

Several limitations in data collection were realised in this study due to the lack of independence between plant samples within species and amongst sites. This is due to the nature of plant distribution within the study site, particularly for Poaceae, which are homogenous in distribution amongst few sites. Sites and transects are not balanced replicates for each plant species measured, and recursion occurred to most but not all selected sites. Further, a lack of equal distribution of all species across all transects due to elephant food plant choices and plant species heterogeneity and distribution also influenced the strength of the data. Third, despite a satisfactory sample size of browsed plants initially, a lack of rebrowsing to plant samples across a variety of species resulted in reduced sample sizes of plants receiving recursion, especially for woody species, and therefore limited statistical power. 
Nevertheless, despite these limitations, I established the likely importance of recovery time for recursion of elephant's bulk food, grass. This was a novel approach for establishing the relationship between resource recovery and recursion by elephants in LKWS by measuring plant recovery rates in an uncontrolled environment. Future studies investigating recursion to plants could be improved by ensuring a relatively even distribution of plant samples across all transects, increasing sample sizes of each species, i.e. more transects or extending sampling distance ( $>2 \mathrm{~m}$ either side of transect) in order to incorporate a larger number of samples within each species for statistical comparison. It would also be beneficial to compare inter-annual variation in re-browsing and elephant impact on their resources as ecosystem engineers. 


\section{Chapter Six}

Plant selection and avoidance by the Bornean elephant (Elephas maximus borneensis) in tropical forest: does plant recovery rate after herbivory influence food choices?

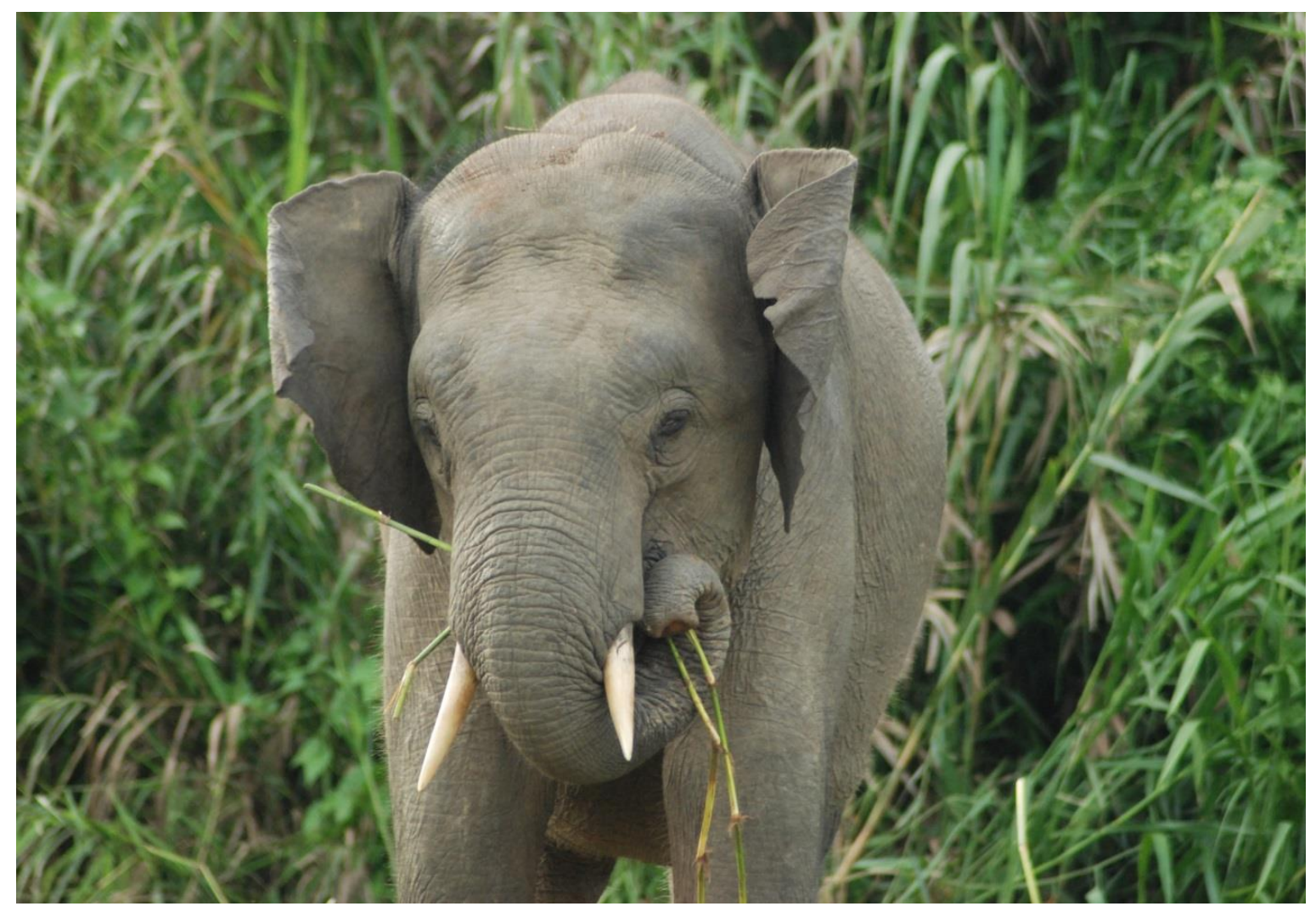

Juvenile male feeding on Phragmites karka, a preferred food, 2011.

Authors note: Chapter Six was published in the Journal of Tropical Ecology July 2014 (Appendix Five). 


\begin{abstract}
The plant vigour hypothesis proposes that herbivores should favour feeding on more vigorously growing plants or plant modules. Similarly, we would expect herbivores to favour plants that regrow vigorously after herbivory. Larger animals, like elephants, may also select plant species relative to their availability and prefer species with larger growth forms in order to meet their intake requirements. The food preferences of the Bornean elephant (Elephas maximus borneensis) in the Lower Kinabatangan Wildlife Sanctuary, Sabah, Malaysia, were investigated along 12 transects in areas where elephants were recently sighted feeding. One hundred and eighty-two plants were eaten and 185 plants were measured for species availability along transects. Species vigour was determined by the monthly regrowth in new shoot length after elephant feeding and the number of new shoots produced on each plant. Measurements were carried out on each plant for 9 months, or until the new shoot was eaten. Plant sizes were determined from their basal diameter. The Bornean elephant did not prefer more vigorous species or species with larger growth forms. New shoots did not grow longer on preferred than avoided species. Additionally, unlike other elephants that live in a forest environment, the Bornean elephant preferred species from the Poaceae (specifically Phragmites karka and Dinochloa scabrida) over other plant types including gingers, palms, lianas and woody trees.
\end{abstract}




\section{Introduction}

The plant vigour hypothesis (PVH) proposes that any plant module, individual, or species that grows rapidly and ultimately reaches a large size relative to the mean growth rate and ultimate size of the population of modules, individual plants, or plant species, suffer enhanced probability of herbivore attack (Price, 1991). Vigorous plants should also grow faster after herbivory to compensate for the damage (Coley et al., 1985; Coley \& Aide, 1990). More vigorous plants have higher nutrient concentrations and less vigorous plants more chemical defences (Price, 1991) and this may be the basis of herbivore foraging decisions. The PVH has been supported in studies on insects (Craighead, 1950; Keen, 1958; Baker, 1972; Furniss \& Carolin, 1977; Price et al., 1987) and vertebrates (Danell et al., 1985; Bergström \& Hjeljord, 1987; Makhabu et al., 2006b).

Herbivore size may influence foraging decisions with respect to plant vigour. Larger herbivores, like elephants, are less selective of plant modules. Rather than responding only to plant chemical or structural properties, a larger herbivore may also respond to plant size (Vivas et al., 1991; Wilson \& Kerley, 2003). Makhabu et al. (2006b) explored the influence of vigour between plant species, rather than within plant species, and found no relationship between the vigour of browse species and feeding preferences of the African elephant (Loxodonta africana). They suggested that the elephant selects whole plants rather than just the new growth. The influence of plant vigour on food selection by Asian elephant species in a rain-forest environment has not yet been investigated.

Elephant species should be less selective than other herbivores. Lower metabolic requirements, larger gut volume and food retention time mean larger herbivores survive on lower-quality food (Bell, 1971; Demment \& van Soust, 1985; Jarman, 1974; Shrader et al., 2012). They can trade food quality for quantity by consuming an abundant, low-value resource instead of searching for less common, higher-value forage (Demment \& van Soest, 1985; du Toit \& Owen-Smith, 1989). For this reason we propose that the PVH is unlikely to explain Asian elephant food choices, but that plants with larger growth forms will be preferred.

Plants have been found to respond both positively and negatively to herbivory. A plant's regrowth rate may increase or, if too much plant material has been taken, decrease because, for example, plant resources are invested instead in the production of anti-herbivore 
defences (McNaughton, 1983). We investigated whether plant size or regrowth vigour after herbivory influence Bornean elephant (Elephas maximus borneensis) preferences.

Herbivore food preferences are reflected by the vegetation chosen in proportion to its availability (Johnson, 1980). Selection-availability or avoidance ratios reveal if animals fed randomly or selectively. Where browse is plentiful it dominates the diet of some forest elephant species (Blake, 2002; Chen et al., 2006; Pradhan et al., 2008; Sukumar \& Ramesh, 1992). But grasses were the major component of the diet of forest elephants throughout the year in Cameroon (Tchamba \& Seme, 1993) and Olivier (1978) found the mainland Malaysian elephant to avoid feeding on woody trees and prefer palms, herbs and grasses. A seasonal deterioration in grass quality has been used to explain a preference for browse by the Asian elephant (Pradhan et al., 2008; Sukumar, 1990).

Based on the literature, we hypothesised that Bornean elephant diet would be dominated by species such as palms, gingers and woody trees rather than plants in the Poaceae, because the habitat types in which Poaceae are found are less common in this tropical forest landscape (Chapter Three). We expect the Bornean elephant, therefore, to select plants proportional to their availability and especially the larger plants that provide abundant biomass. Due to an ability to feed on lower-quality food, because of its body size, we do not expect plant vigour to influence the elephant food preferences in the Lower Kinabatangan Wildlife Sanctuary.

\section{Methods}

\section{Study site and focal species}

See general Introduction, pages 26-28.

\section{Vegetation sampling}

Sampling sites were selected opportunistically throughout the elephant's home-range. These sites represented the elephant range used by the herds in March-June 2011, which is early dry season (Figure 1). We searched sections of the sanctuary from the river and tracks for elephants and recent elephant signs. Signs included fresh dung, urine, fresh footprints and recently browsed plants.

We established $50 \mathrm{~m}$ transects at places where elephants were feeding. The transects were $>300 \mathrm{~m}$ apart. One transect was established per day. We tracked fresh elephant signs 
including footprints, dung and signs of feeding to establish the transect along the group feeding path. All plants showing signs of elephant feeding within $2 \mathrm{~m}$ either side of transects were marked and labelled with the date and a reference number. Samples of all plant species were collected for identification at the Sabah Forestry Department Herbarium (SAN), Sandakan.

The height and basal diameter of the stem showing the feed signs were measured to establish the size and relative age of the plants selected by the elephants. Measures of availability were taken at 5-m intervals along the transect, where the species closest to the transect was recorded. Transects and marked plants were revisited and regrowth measured each month from April 2011 to December 2011. If the plant died or had been re-browsed by elephant or other herbivores, thus preventing measurements of regrowth, this was also recorded.

Selected plants had diverse growth forms and responded to herbivore feeding by recovering in different ways. Monthly regrowth measurements were taken on a selected new shoot closest to the node nearest the feed sign, or from the plant base, depending on how the plant recovered. Only recovery visible above the ground and within a $30-\mathrm{cm}$ radius of the focal plant was measured. Measurements included new shoot growth in length, basal diameter of the new shoot and a count of the number of new shoots produced each month. 


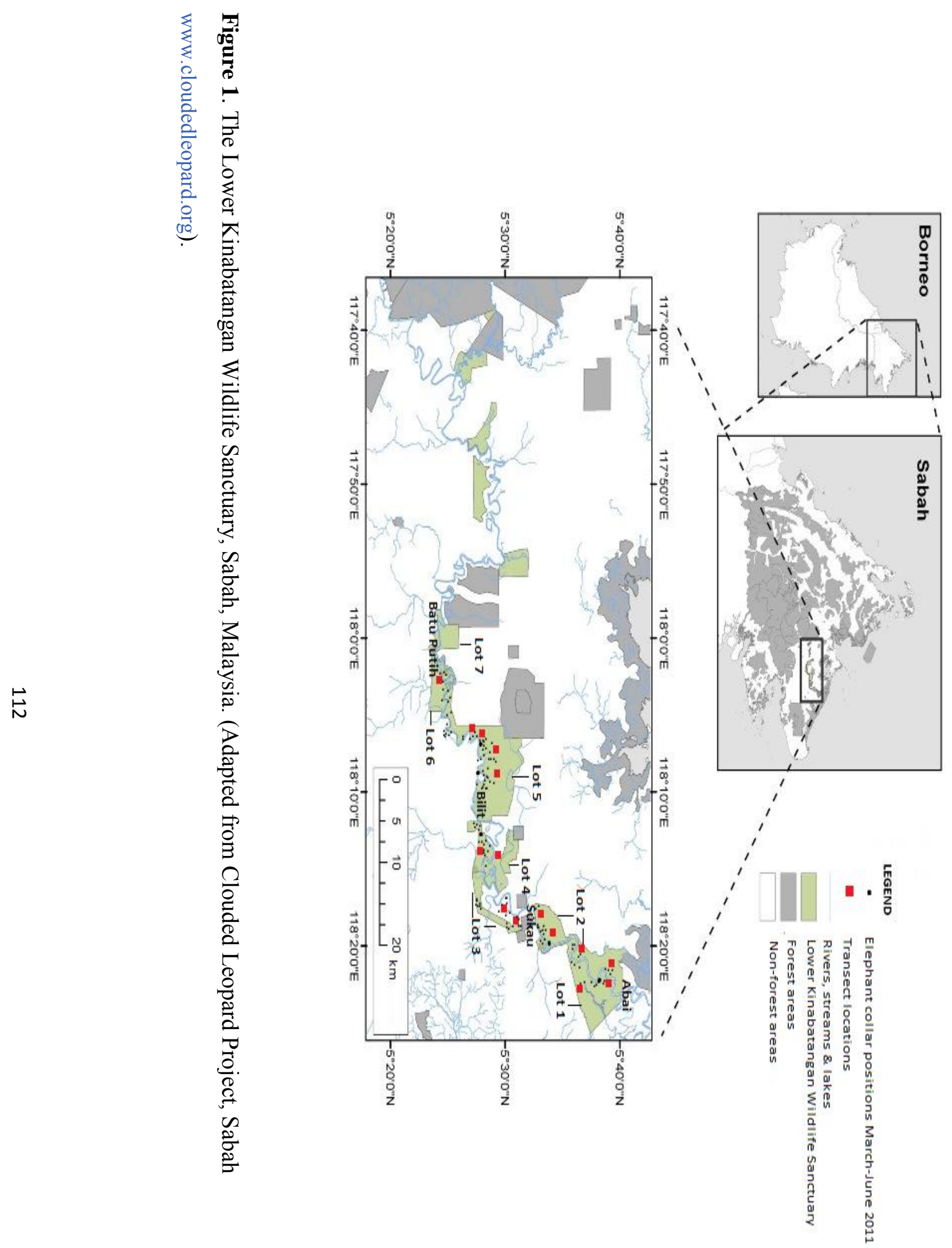




\section{Data analysis}

\section{Plant species preference}

Plant species preference was calculated using the relative availability (RA) of each species compared to their relative use (RU) by the Bornean elephant. We focused only on frequently encountered species by limiting the preference analysis to those species for which more than five individual plants were sampled. Species and sample sizes included in analysis are listed in Table 1.

$$
\mathrm{RA}=\frac{N a}{\mathrm{Ta}}
$$

$\mathrm{Na}$ is the number of available plants of a given species and $\mathrm{Ta}$ is the number of available plants across all species (185).

$\mathrm{RU}=\frac{N u}{T u}$

$N u$ is the number of times a species was selected. $T u$ is the total number of plants selected for feeding across all species (182).

Preference ratio $=\frac{R U}{R A}$

Species with a preference ratio $>1$ were selected and those $<1$ were avoided (Petrides, 1975). The ratios were then converted to binary numbers where selected $=1$ and avoided $=0$.

\section{Grass and browse selection}

Chi-square tests between grass, bamboo, palms, Zingiberales (ginger hereafter), lianas and woody trees were used to determine if the differences between use and availability were significant. Statistical analyses were undertaken using SPSS 18.0.

\section{New shoot volume}

The vigour of each species was determined by taking averages of (1) the average number of new shoots, (2) the average values of change in new shoot length, and (3) the average growth in basal area of the new shoot for each species between months. These were then used to 
calculate the average new shoot volume for each species using the formula for a cone (V= $\frac{\pi r^{2} h}{3}$

$=($ shoot basal area $\mathbf{X}$ average monthly shoot length $) / 3 \times$ average number of new stems $=\frac{\pi r^{2} L}{3} \times S$

Where $\pi r^{2}$ is the basal area, $\mathrm{L}$ is the average growth in shoot length per month and $\mathbf{S}$ is the average number of new stems produced by the plant per month.

\section{Plant vigour and size}

The variables for plant vigour included the maximum monthly average for each species of growth in length of the new shoot (mm), number of new shoots produced and volume of new shoots $\left(\mathrm{mm}^{3}\right)$. Poaceae were not included in the vigour analyses and species with sample sizes of less than five were removed. A Principal Component Analysis (PCA) was used to reduce confounding effects of partial correlation between variables of plant regrowth vigour, thus reducing the number of covariates in the model to two. The principal component with which volume was most strongly associated had an eigenvalue $<1$ and was therefore removed from the regrowth vigour analyses. The length of new growth and number of stems produced was analysed using a Generalised Linear Model (GLM) with the preference ratio, a binary logistic, as the dependent variable using SPSS 18.0. The regrowth vigour of woody trees was first analysed to compare to previous studies, then vigour of gingers, palms and lianas was included in analyses because these other plant types being available to elephants in a rainforest environment. Plant size, using the basal diameter of the main stem (mm), was also analysed using a GLM with the preference ratio as the dependent variable.

\section{Results}

A total of 182 plants were eaten and 185 available plants were measured. Eighteen species were included in the analyses. Plant species were grouped into plant types for used and available comprising Poaceae (Phragmites karka, a grass and Dinochloa scabrida, a bamboo), gingers, palms, lianas and woody trees (Figure 2). 


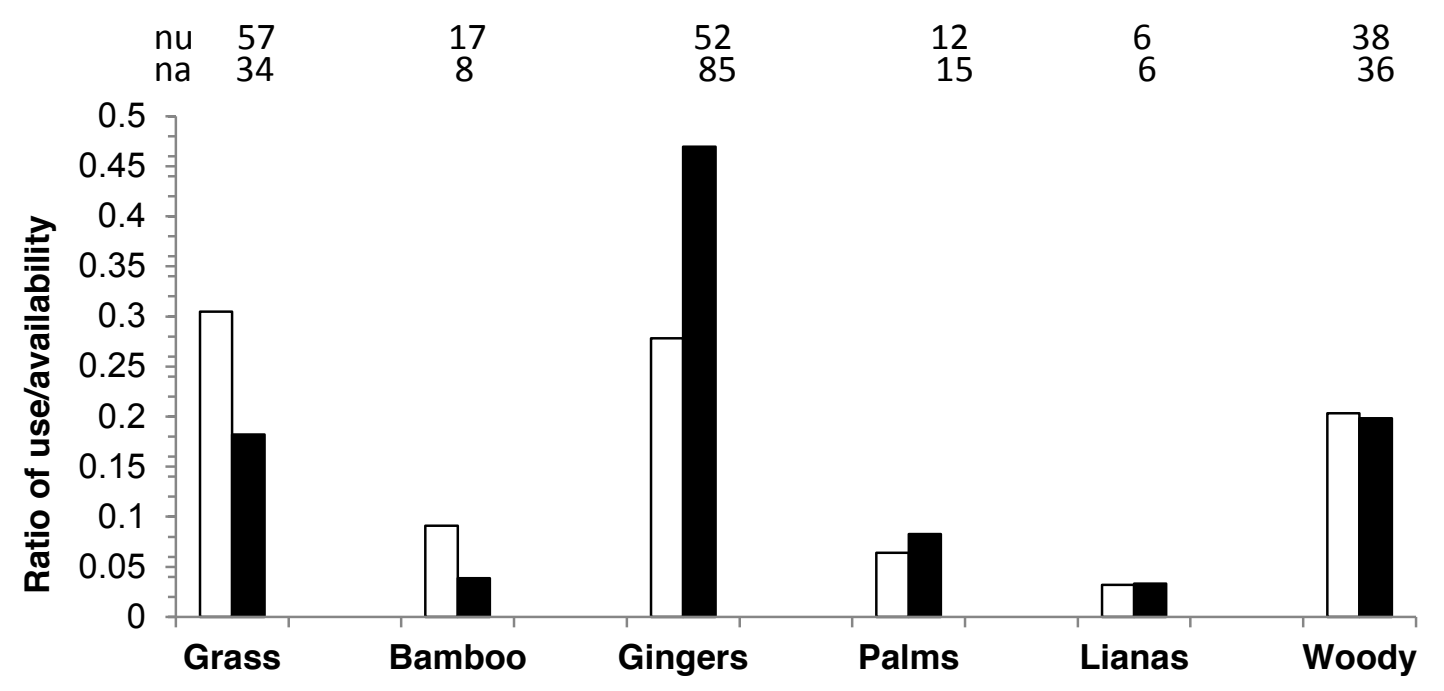

Figure 2. The ratio of plants selected by the Bornean elephant and plant availability in the Lower Kinabatangan Wildlife Sanctuary, Sabah. Plant species are in plant groups (grass, bamboo, gingers, palms, lianas and woody species). Used ratios of plants are white bars and available ratios of plants are black bars. The number of individual plants used (nu) and number of individual plants available (na) for each plant group are shown. Grasses are Phragmites karka; Bamboo Dinochloa scabrida; gingers include Alpinia ligulata, Costus speciosus and Donax canniformis; palms include Licuala sp., Arenga sp. and Daemonorops sp.; lianas include Fordia splendidissima and woody includes Memecylon sp., Dillenia excelsa, Gardenia elata, Syzygium sp., Bridelia stipularis, Mallotus muticus, Lepisanthes fruticosa, Alangium javanicum and Garcinia parvifolia.

The elephant favoured the grass and bamboo more than other plant types (Pearson chi-square, $\left.\mathrm{N}=18, \chi^{2}{ }_{1}=0.920, \mathrm{P}=0.012\right)$ although these were less common in the landscape (43 available Poaceae samples) compared to other species (142 available samples). The elephant preferred six and avoided ten of the most common species along transects (Figure 3). Palms such as Licuala sp., and gingers such as Costus speciosus and Donax canniformis, were selected. Woody trees were less common, but Garcinia parvifolia, Lepisanthes sp. and Alangium sp. were most abundant and selected. 


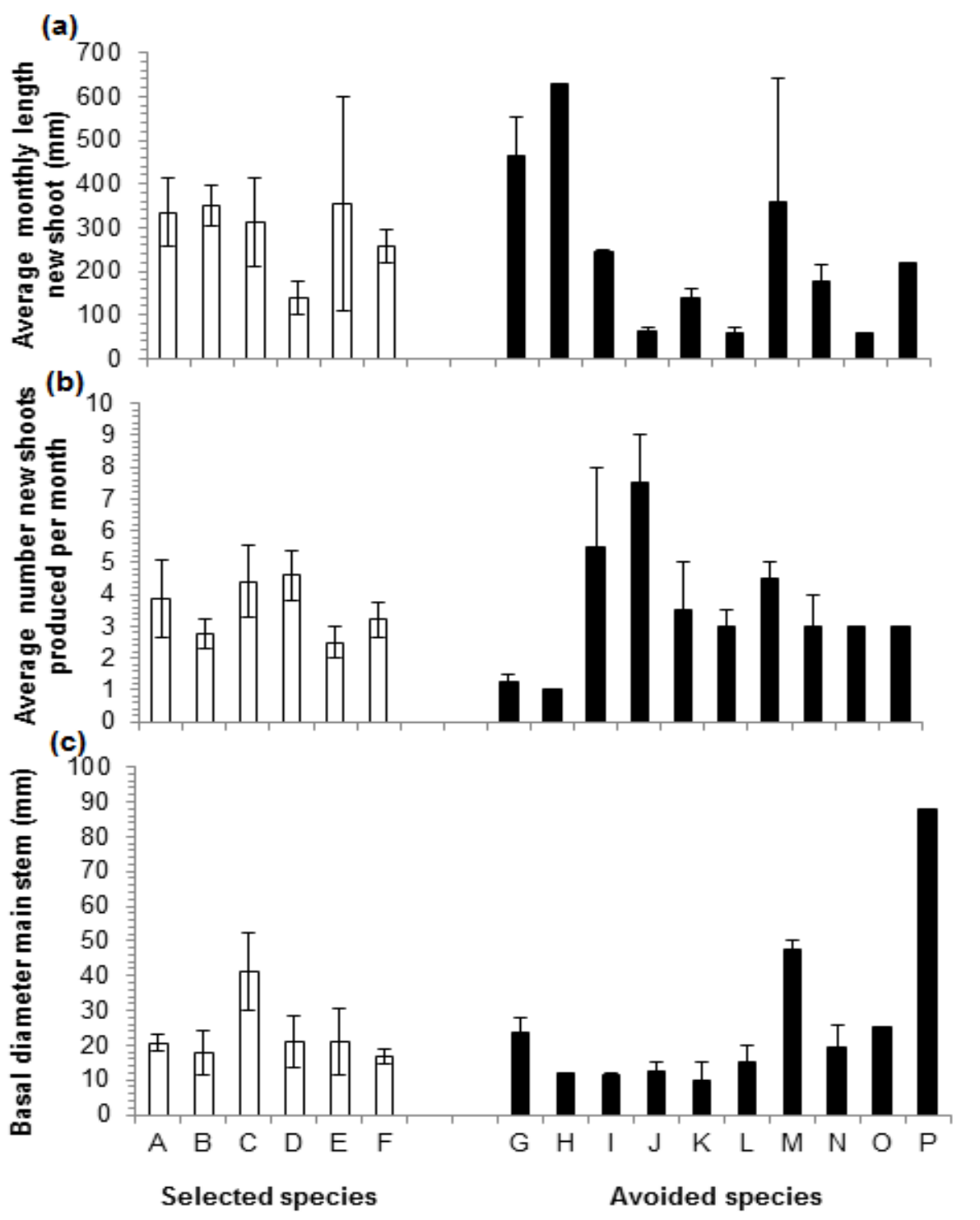

Figure 3. Plant selection and avoidance by the Bornean elephant in the Lower Kinabatangan Wildlife Sanctuary, Sabah, related to regrowth vigour for each species. Includes the average monthly growth in new shoot length ( $\mathrm{mm}$ ) (a) average monthly number of new shoots (b) and plant size using average basal diameter of the main stem $(\mathrm{mm})(\mathrm{c})$. Error bars represent standard error of individual samples within a species. Selected species in white and avoided species in black. Letters correspond to species names given in Table 1. 
Table 1. Plant species selected by Bornean elephant and available along transects within the Lower Kinabatangan Wildlife Sanctuary, Sabah, Malaysia. Plant species are included in analyses for Figures 2 and 3 and were identified by Sabah Forestry Department herbarium (SAN), Sandakan, Sabah.

\begin{tabular}{|c|c|c|c|}
\hline $\begin{array}{l}\text { Plant group } \\
\text { (Figure 2) }\end{array}$ & Family & Species & $\begin{array}{c}\text { Total } \\
\text { sample size }\end{array}$ \\
\hline
\end{tabular}

\begin{tabular}{|c|c|c|c|c|}
\hline Grass & Poaceae & $\begin{array}{l}\text { Phragmites karka (Retz.) } \\
\text { Steud. }\end{array}$ & 91 & - \\
\hline Bamboo & Poaceae & Dinochloa scabrida S. Dransf. & 25 & - \\
\hline Ginger & Zingiberaceae & Alpinia ligulata K. Schum. & 49 & G \\
\hline Ginger & Costaceae & Costus speciosus J. Koenig & 12 & A \\
\hline Ginger & Marantaceae & Donax canniformis K. Schum. & 76 & $\mathrm{~F}$ \\
\hline Palm & Arecaceae & Licuala sp. & 11 & B \\
\hline Palm & Arecaceae & Arenga sp. & 9 & $\mathrm{H}$ \\
\hline Palm & Arecaceae & Daemonorops sp. & 7 & I \\
\hline Liana & Leguminosae & $\begin{array}{l}\text { Fordia splendidissima (Blume } \\
\text { ex Miq.)J.R.M Buijsen }\end{array}$ & 12 & $\mathrm{~J}$ \\
\hline Woody & Melastomataceae & Memecylon sp. & 14 & $\mathrm{~K}$ \\
\hline Woody & Dilleniaceae & Dillenia excelsa (Jack) Gilg. & 11 & $\mathrm{~L}$ \\
\hline Woody & Rubiaceae & Gardenia elata Ridl. & 9 & M \\
\hline Woody & Myrtaceae & Syzygium sp. & 10 & $\mathrm{~N}$ \\
\hline Woody & Phyllanthaceae & Bridelia stipularis Blume & 9 & $\mathrm{O}$ \\
\hline Woody & Euphorbiaceae & $\begin{array}{l}\text { Mallotus muticus (Muell. } \\
\text { Arg.)Airy Shaw }\end{array}$ & 9 & $\mathrm{P}$ \\
\hline Woody & Sapindaceae & $\begin{array}{l}\text { Lepisanthes fruticosa. } \\
\text { (Roxb.)Leenh. }\end{array}$ & 10 & $\mathrm{D}$ \\
\hline Woody & Guttiferae & $\begin{array}{l}\text { Garcinia parvifolia (Miq.) } \\
\text { Miq. }\end{array}$ & 8 & $\mathrm{C}$ \\
\hline Woody & Alangiaceae & $\begin{array}{l}\text { Alangium javanicum } \\
\text { (B1.)Wang. }\end{array}$ & 6 & $\mathrm{E}$ \\
\hline
\end{tabular}


The Bornean elephant did not select woody trees that recovered more vigorously (GLM Logistic Regression, Wald Chi-square, $\mathrm{df}=5$ : new shoot length $\chi^{2}{ }_{1}=1.17, \mathrm{P}=0.278$; number of new stems $\chi^{2}{ }_{1}=0.479, \mathrm{P}=0.489$ ), nor did it prefer more vigorously regrowing species when more plant forms were included in analyses such as gingers, lianas and palms (GLM Logistic Regression, Wald Chi-square, $\mathrm{df}=12$ : new shoot length $\chi^{2}{ }_{1}=0.598, \mathrm{P}=0.439$; number of new stems $\chi^{2}{ }_{1}=0.231, \mathrm{P}=0.631$ ). Plant size did not influence selection (GLM Logistic Regression, Wald Chi-square, $\mathrm{df}=13$ : basal diameter $\chi^{2}{ }_{1}=0.117, \mathrm{P}=0.733$ ). Favoured species were therefore not larger, their new growth not longer, nor did they produce more new stems than avoided plants.

\section{Discussion}

We expected the Bornean elephant to select plants proportional to their availability and prefer palms, gingers, lianas and woody trees over grasses, including bamboos. We proposed that plant size, rather than plant regrowth vigour, might influence food choices. We did not find support for the influence of plant regrowth vigour or plant size on elephant food-plant choices but we did determine that the elephant in LKWS preferred to feed on Poaceae (Phragmites karka and Dinochloa scabrida) proportionately more than their availability, compared to other measured species.

\section{Plant selection}

Bornean elephant in the LKWS fed mainly on a subset of available species. Food-plant selection was not explained by the relative abundance of plant species. This finding is consistent with several other studies on the feeding behaviour of African elephant (Loxodonta africana), African forest elephant (Loxodonta cyclotis) and Asian elephant (Elephas maximus) (Codron et al., 2010; McKay, 1973; Olivier, 1978; Seydack et al., 2000; Short, 1981; Sukumar, 1990; Williamson, 1975; Wing \& Buss, 1970).

Most studies of other elephant taxa (Loxodonta cyclotis) (Blake, 2002) and (Elephas maximus) (Sukumar, 1990) describe their preference for browse unless grass was the dominant vegetation, whereas Tchamba \& Seme (1993) and Olivier (1978) found grasses to be preferred despite being less abundant. In LKWS, although open grassed areas along forest margins (containing early-successional species such as bamboo) and riverine areas (containing semi-aquatic grasses such as reed), cover just 14\% of the landscape (compared to 
approximately $60 \%$ forested areas and 26\% swamp; English, unpubl. data). The Bornean elephant preferred feeding on the two Poaceae (Phragmites karka and Dinochloa scabrida) in open grassed areas along forest margins, than on other species in the study. Thus, there is variation in the literature of the importance of grass species in the diet of the forest elephant relative to its abundance.

Asian elephants are believed to switch their diet preferences from grass to browse depending on seasonal changes in plant quality. For example, browse is consumed more in the dry season and grass in the early wet season (Sukumar, 1990); although some other studies found elephants to prefer grasses despite seasonal influences (Olivier, 1978; Tchamba $\&$ Seme, 1993). Our study period was in the dry season when more browsing might be expected but still the Bornean elephant preferred to feed on the two Poaceae mentioned. Seasonal influence is unlikely to be a major factor influencing plant quality in our study site because, despite a wet and dry season, rainfall is common throughout the year. Moreover, one of the species (Phragmites karka) is semi-aquatic and found near a permanent water source. Moreover, these species are also perennial. It is unknown if there were any natural open areas within the forest historically in Sabah. Logging is believed to have started at least a century ago and the area could have been inhabited for many centuries by nomadic villages and the forest cleared for farming purposes. The river itself has been affected by a series of disturbances due to flooding and natural processes decimating forest and replacing with open areas containing early-successional species such as grasses, including bamboos (R. Nilus pers. comm.). However, regardless of their origin, these areas provide the elephants with much of their preferred food plants.

A second scenario that might explain why the Bornean elephant prefers Poaceae in the LKWS is that the quality of other plants within the elephant herd's range may be poor, because the remnant habitat is poor or the overall habitat quality may have declined. Higher quality habitat may have been replaced by oil palm cultivation (Elaeis guineensis) and this may have forced the elephant to use areas that may be less optimal for foraging, or it may be exceeding the forest's capacity to support it. An increase in the LKWS elephant population in the last 10 years ( $<100->200$ individuals) (Ancrenaz pers. comm.) may have depleted preferred browse species and increased its use of Poaceae species to compensate. Bulk feeding on species such as reed and bamboo may enable elephants to meet intake requirements cannot be met by more abundant species.

Another scenario involves the influence of river hydrology on soil and plant quality through sediment and nutrient deposition by water filtered through the riparian zone. Rain- 
forest soil quality can range from highly leached, infertile soils to fertile, less-weathered, alluvial soils (Ashton, 2004). Grasses, such as the common reed, in a floodplain landscape may be preferred due to the soil quality in riverine areas having higher nutrient concentration compared to soils further from the river where browse species are found. Moreover, vegetation growing on nutrient-deficient soils has been found to contain more chemical defences compared to plants found in areas with nutrient-rich soils (Coley et al., 1985; OwenSmith \& Cooper, 1987). Therefore, some plant species may be relatively high in chemical defences and of poorer quality to elephant within our study site. In addition, regular feeding increases soil nitrogen cycling and denitrification from herbivore dung deposition and urine, leading to elements being returned to the soil in readily available forms (McNaughton et al., 1988; Ruess, 1986; Risser \& Parton, 1982). The result is improved nitrogen availability for plants in these areas (Hamilton \& Frank, 2001; Holland \& Detling, 1990; McNaughton et al., 1997; Risser \& Parton, 1982). Whatever the cause of plant preference by the Bornean elephant in LKWS it is clear that the common reed and bamboo are a significant part of its diet in a way not previously appreciated.

\section{Plant regrowth vigour and size}

The Bornean elephant of the LKWS did not prefer more vigorously regrowing plant species. This finding supports predictions based on body size and metabolism where the elephant represents an upper extreme in its tolerance of lower-quality food compared to smaller herbivores (Bell, 1971; Demment \& van Soest, 1985; Jarman, 1974). For a larger animal that can accept a lower-quality diet, almost the whole plant is a homogenously acceptable food item, whereas for a smaller animal requiring a higher-quality diet a plant is a set of heterogeneous parts, from among which the more nutritious components must be selected (Bell, 1971; Jarman, 1974). High-quality parts of plants generally form smaller food items than do the low-quality parts. Thus, it is fitting that the diet selection of elephant, which must select their food for quantity rather than quality, should not be influenced by selection for more vigorous plants which are generally of higher nutritional quality (Price, 1991).

Previous studies have found conflicting results in plant size preferences of elephants. The African forest elephant has been reported to prefer woody species with a smaller diameter at breast height (dbh) (Blake \& Inkamba-Nkulu, 2004; Wing \& Buss, 1970). In contrast, the Bornean elephant shows a preference for larger dbh in one woody species (Macaranga sp.) (Matsubayashi et al., 2006). Compared to small herbivores, an elephant may 
consume more modules of a plant as it has a larger bite size and use of a prehensile trunk. Thus elephant might respond positively to a plant's size because more edible components are available (bark, leaves, new shoots, fruit and roots) (Vivas et al., 1991; Wilson \& Kerley, 2003). We expected the Bornean elephant to prefer larger plants as part of its optimal foraging strategy, as they should be less selective and conserve energy rather than seek resources elsewhere (Charnov, 1974). However, we found that selection and avoidance was not influenced by the size of the plant.

Our results suggest Bornean elephant foraging behaviour occurs at a larger spatial scale than the plant level. Shrader et al. (2012) proposed that because elephant were generally forced to feed less selectively to provide sufficient intake, it is possible that they will make foraging decisions at the habitat or site level rather than at the individual plant level and this may also be the case with the elephants in our study.

\section{Implications for management of habitat and population}

Our findings about Bornean elephant food choices have direct implications for the restoration and management of elephant habitat in Sabah. Our results suggest that preservation of open areas along forest margins where Dinochloa scabrida is common and riverine areas where Phragmites karka is common is necessary for elephant conservation. Some forest disturbance is not deleterious as increased sunlight in ecotone and open areas encourages growth of earlysuccessional species such as those preferred by the Bornean elephant. Currently open areas along forest margins are actively planted with tree species as this is considered the foundation state for restoration. We recommend, however, that wildlife and habitat rehabilitation managers set aside some open areas for Poaceae throughout the LKWS. Replanting of trees along the river bank is important for minimising erosion and providing opportunities for food or movement to a number of species, such as primates. It is also visually appealing for ecotourism in the area. The importance of bamboos and reeds, and perhaps other species within Poaceae, for the Borean elephant, however, should be incorporated into management and restoration planning. 


\section{Acknowledgements}

Thank you to Victoria University of Wellington for supporting the study and providing a doctoral scholarship and a completion scholarship for Megan English. Thank you to the Economic Planning Unit and Sabah Wildlife Department for permitting the research in the Lower Kinabatangan Wildlife Sanctuary. Research was made possible by field assistants provided by HUTAN Kinabatangan Orang-utan Conservation Project and their Elephant Conservation Unit. Thank you also to Danau Girang Field Centre for providing a vehicle and field staff and to Dr. John Sugau at the Sabah Forestry Department Herbarium, Sandakan for identifying plant species and to Prof. Phillip Garnock-Jones from Victoria University of Wellington. This project was supported by funds to Megan English and Dr. Wayne Linklater from Zoos Victoria, Australia. 


\section{Synopsis}

I chose to investigate recursion ecology in the elephant because of the potential to test hypotheses for food-plant preferences, foraging strategies, and recursion for an animal in which it has not yet been studied and which represents the uppermost extreme in a herbivoreresource relationship.

Elephas maximus borneensis exist in an environment where available habitat continues to diminish due to agricultural development and expansion. Consequently these elephants are under increasing pressure and human-elephant conflict is rising (Othman et al., 2013). Their need for a large quantity of food but restriction to a fragmented habitat of forest patches surrounded by encroaching agriculture, requires an understanding of their resource needs, essential for designing an effective management strategy to promote habitat maintenance and to ensure the survival of the species in the wild.

My research has contributed to the available literature not only of this rare sub-species but of large herbivore-resource relationship studies in general using techniques that can be applied across a variety of species and habitat types. Studies of the temporal variation of large herbivore-resource relationships on wild animals are few in number and limited in scope. I evaluated elephant habitat preferences and recursion to foraging sites and determined that the foraging strategy of the elephants reflected the quality of the site, the extent of previous use, and the time since its last use. This approach introduced a novel technique to identify critical resources for the elephant population by highlighting the importance of open grass areas along forest margins which also contain the elephant's preferred foods, grasses and bamboos. Recursion occurred over two main time-scales, short-term (within 48 hours) and long-term (after 151-250 days), indicating two foraging strategies (English et al., 2014b). I identified that elephants re-browse on grasses only after they have recovered and that recursion to areas containing this food is most strongly explained by recovery rates. My study identified how elephant food-plant preferences differ in LKWS from what was expected of a forest elephants, as well as tested established hypotheses of herbivore food-plant preferences associated with plant vigour and size (English et al., 2014c). Elephants in LKWS preferred grass to browse despite the former being less abundant across the landscape, and selection of browse was not influenced by plant size or vigour. 


\section{Review and synthesis of recursion knowledge}

Recursion by herbivores has not been extensively studied even though it has important implications for ecology and for population and habitat management. Nevertheless, studies of recursion might be informed by better studied topics that use different terminology like site reuse, or topics which have important similarities such as spatial memory and foraging and foraging site-fidelity. A lack of literature that has covered these topics in large herbivores was evident when compared to the relatively abundant literature for other vertebrates such as birds, small mammals, primates and fish. Studies of resource-use over varying temporal scales, that would be capable of testing for recursion and its implications, were few in wild animals and uncontrolled environments.

Existing studies of site recursion have investigated its frequency, duration and occurrence over varying time-scales, but few have incorporated foraging theory and investigated the reuse of individual plants. In order to understand what influences recursion frequency, the time spent at a site, and over what time-scales recursion occurs, site characteristics and resource recovery rates should be incorporated into a study of recursion to provide a detailed understanding of the influences on an animal's foraging strategy over varying spatial and temporal scales. My review and synthesis of the literature therefore guided the design of the empirical chapters.

\section{Recursion at two scales: site and plant}

Compared to small herbivores, large herbivores require a higher quantity, rather than quality, of resources in order to meet their intake requirements. We expected recursion, therefore, to be less common in elephants than smaller mammals due to the latter benefiting from acquiring food in its highest productivity stage, such as from new growth. However elephants might recall the spatio-temporal distribution of quality areas better than a smaller herbivore such that an elaborate recursion foraging strategy is possible.

Repeated use is not only associated with facilitation of quality resource production, such as in the development of grazing or browsing lawns where positive feedback occurs between grazing/browsing and plant palatability (McNaughton, 1984,1985; Archibald, 2008; Cromsigt \& Olff, 2008; Mouissie et al., 2008), but is also a result of spatial predictability and an animal's ability to relocate profitable areas. If elephants do, therefore, demonstrate 
recursion in order to maximise feed intake and minimise energy expenditure then they should return to sites only after resources have replenished, or if they return sooner, use resources within a site sparingly at each visit. If elephants are not foraging optimally, such as when recursions occur to areas where resources have previously been exhausted and before resource recovery, then this indicates resources are inadequate or are at risk of being overutilised. This could be a sign that the elephants are exceeding the carrying capacity of their environment or modifying vegetation structure and composition. If elephants do forage optimally then recursion was expected to occur as a function of the quality of the site, extent of its last use, and time since its last use as these factors influence site resource status and resource recovery. If more time was spent at recursion sites compared to sites that were not returned to then recursion sites should be considered high quality for elephants.

Recursion to foraging sites did occur by elephants in the LKWS and was common at two main temporal scales. This indicated that there were two different foraging strategies associated with recursion: one with site sampling and the other with plant recovery time. The number of days between the first visit and recursion increased and decreased in proportion to how long the site was utilised previously. The more time spent at the site initially, the more days passed until the elephants returned. If less time was spent at the site then fewer days passed until recursion. These results suggest a degree of spatial and temporal awareness and that the elephants manage their resources by returning after a longer period if the site was more heavily utilised, or returning after less time if the site was used sparingly.

Elephants have been found to have a high degree of spatio-temporal awareness (Hart et al., 2008) and my observation that the timing of returns to sites depended on the degree of last use indicates that the Bornean elephants are applying an elaborate spatio-temporal map of foraging sites. Moreover, the time spent at a site during a recursion event matched the time spent there earlier. A relationship between the amount of time herbivores allocate to specific areas and the resource quality of the area had been identified previously (Bailey et al., 1996). The elephants in LKWS spent more time within sites to which they later returned than within sites they did not revisit. This suggests that recursion sites are favourable to the elephants and of a high importance to their resource needs. The habitat type of a foraging site also influenced how much time was spent there during recursion, with the elephants spending more time within open grass areas along forest margins compared to other habitat types. This was also their preferred habitat type determined from the use:availability analysis. This habitat type also contained their preferred foods such as the grass species identified in Chapter Six, Dinochloa scabrida and Phragmites karka as well as other species in the 
Poaceae. This result reiterates the importance of open grass habitat that contains preferred food plants and further supports the likelihood that a study of recursion can identify critical habitat.

A previous study had suggested that plant recovery rates influence recursion (BarDavid et al., 2009), although it had not yet been explored systematically in wild animals. Where longer-term recursions occurred after 151-250 days, these returns were likely to coincide with plant recovery. To measure plant recovery until recursion was the next step in an attempt to understand foraging strategies and determine whether or not elephants forage optimally or over-utilise their resources by re-browsing before plants have recovered (Chapter Five).

Plant recovery time and plant species had a strong influence on the timing of recursions to individual plants. Recursion rates were greatest to their preferred species, confirming the importance of Poaceae, in particular Dinochloa scabrida and Phragmites $k a r k a$, in the elephant diet. In all cases, Poaceae plants were returned to and were re-browsed only after they had recovered. Other browse species such as woody plants, palms and gingers were either not re-browsed or, for the small number of samples from those plant types, were re-browsed before they had recovered to their previous size. Again, this result confirms the importance of the grasses in the elephant diet in LKWS; it is their preferred diet and provides the bulk of their intake. The results also suggest that elephants do not over-utilise their preferred resource that also forms the bulk of their diet and that their returns coincide with resource (plant) recovery from feeding. Furthermore, the recovery time of three of the elephant's preferred plants (one grass species and two ginger species, Chapter Six, Table 1) occurred between 151-250 days. Two palm species, two liana species and three woody species eaten by elephants also had a recovery time of between 150-250 days (Chapter Five, Table 1 and Chapter Six, Table 1) thus supporting the suggestion that resource recovery influences long-term recursion because recovery times and recursion timing correspond.

When my results are related back to the discussion of potential foraging strategies in the recursion synthesis in Chapter 2 (GUD and IFD), it appears that (a) short-term recursion indicates a strategy that resembles foraging with imperfect knowledge and perhaps reflects information gathering, while (b) long-term recursion to preferred, bulk-feeding plants (grasses) resembles a strategy of foraging with perfect knowledge and indicates optimal foraging. Elephants also spent greater time at recursion sites compared to non-recursion sites, which suggests that site quality influences foraging decisions and therefore elephant foraging follows the Ideal Free Distribution in time as well as space (Charnov, 1976). My results also 
indicate that the current elephant population is within capacity of the plant resources to support it.

\section{Advancing our understanding of animal food-plant relationships}

More vigorously growing plants are often of higher nutrient quality and are therefore preferred by many herbivore species (Craighead, 1950; Keen, 1958; Baker, 1972; Furniss \& Carolin, 1977; Danell et al., 1985; Price et al., 1987; Bergström \& Hjeljord, 1987; Price, 1991). Slower growing species often invest more time in producing physical and chemical herbivore deterrents (Price, 1991). However, because elephants require large quantities of food rather than food of high quality, I hypothesised that plant size (Vivas et al., 1991; Wilson \& Kerley, 2003) rather than plant vigour would govern the food plant preferences of browse species. I found that neither size nor vigour influenced elephant browse preferences of woody species, lianas, gingers and palms.

Elephants are believed to be generalist herbivores that tend to select species proportional to their availability. My results, however, indicate that the Bornean elephant is selective of plant species, even if plant vigour and size did not influence preference. Where grasses are uncommon browse species dominate the diet of many forest elephant taxa (Blake 2002, Chen et al. 2006, Pradhan et al. 2008, Sukumar \& Ramesh, 1992). In LKWS, however, elephants preferred to feed on grass species despite these being less abundant across the landscape compared to other species they selected for feeding. This result is consistent with findings about African elephant plant preferences in Cameroon (Tchamba \& Seme, 1993) and Asian elephant preferences in peninsular Malaysia (Olivier, 1978). This highlights the importance of bulk feeding on grasses and reiterating the importance of protecting habitat containing these plant types, and ensuring they are accessible to the elephants.

Elephant preference of open grass areas along forest margins (Chapter Three) recursion and increased time spent at sites within this habitat type (Chapter Four), and recursion occurring to grasses, their preferred food, only after they have recovered (Chapters Five and Six), emphasises the importance of grass in the elephant diet and the influence of plant recovery after herbivory on recursion timing and intensity. Recursion behaviour also suggests that elephants are facilitating and engineering grass growth within scattered sites in LKWS despite these areas being less abundant than sites containing browse food-plants. 
In summary, my study advances knowledge of the behaviour and ecology of the rare Bornean sub-species of elephant and contributes to our understanding how herbivore recursion may be a foraging strategy for optimising resource-use. Bornean elephant, and other large herbivores, may benefit by returning to known high-quality sites, such as those that contain preferred resources like grass and bamboo, and after sufficient time has passed since their last utilization to allow for those resources to recover. This enables the consumption of larger quantities of bulk-food plants. On the other hand, not being able to relocate profitable sites or returning to non-profitable sites, such as those that contain poorer quality or quantity of resources, would be costly to a herbivore. I tested whether elephant in LKWS were foraging optimally by exploring whether site quality influences recursion and if plant recovery influenced recursion time. I expected more time spent at these sites per visit, that time between visits would correspond with time spent at the site previously, and that these sites would contain preferred resources. I also expected the time to recursion to correspond with individual plant recovery. These expectations were met in Bornean elephant amongst sites and preferred food plants. Foraging recursion could be profitably applied to other herbivore species at various spatial scales and in different environments to advance our understanding of optimal foraging.

\section{Conservation implications}

To ensure ongoing management of large herbivore populations their species-specific resource needs must be understood. Studies of recursion are able to identify resource-use over short and long term temporal scales. Such studies can provide more insight into habitat use and resource requirements, and can tell us whether a species is over-utilising its resources. In addition, the findings can be used to foster species conservation by identifying profitable sites and plants.

I identified the importance of the few scattered open grass areas which provide the elephants with their preferred food. They are also the areas that received most recursions. Access to these areas must be ensured for the elephant population in LKWS. I recommend to planners involved in elephant conservation that forest rehabilitation projects incorporate the elephant's preferred food plants and habitat as part of a landscape management strategy. If access to these areas is inhibited or prevented by either natural or anthropogenic influences, elephants may seek resources from alternative and potentially more risky areas (Estes et al., 2012). For example, an increase in foraging within agricultural areas and the ecotone habitat 
bordering these areas might be expected, thus increasing the likelihood and intensity of human-elephant conflict and subsequently jeopardising the longevity of this species in the wild. If the elephant population continues to grow or habitat availability continues to decline I would expect an increase in the rate of recursions to sites containing the elephants preferred food plants before these plants have sufficiently recovered. Moreover I would also expect an increase in the use of poorer quality sites such as those sites containing avoided food plants, or sites with higher levels of human disturbance. If interspecific competition increases as a result of a growing elephant population, resources may become limited and I would therefore expect herd sizes to decrease, which would be expected to lead to more frequent recursions by smaller groups.

A study of recursion ecology not only improves our understanding of animal habitat use and food-plant relationships over varying time scales, but can be used to inform species conservation by giving a broader perspective of animal resource needs. The wider implications of understanding recursion can lead to identifying what influences the formation of home-ranges and migration routes, informed by the distribution and location of critical resources identified in recursion studies. Wildlife practitioners and managers may then incorporate this information into the design of reserves and the location or placement of corridors linking these profitable areas, recursion resources and sites. Furthermore, patterns of herbivore recursion will contribute to understanding animal food-plant relationships and the influence of recursive behaviour on plant-community dynamics.

My thesis has achieved new knowledge on the Bornean elephant, tested long-standing hypotheses for food-plant preferences, and investigated a rarely considered topic for wild animals in natural settings - recursion. Recursion proved to be an important characteristic of elephant range use with direct relevance to understanding elephant ecology and the steps necessary to ensure elephant population and habitat are managed in order to meet their resource needs.

\section{The future in recursion studies}

Future studies of recursion could build on my study by measuring this behaviour over a much longer period, we could address the following knowledge gaps: Do species exhibit longer, inter-annual cycles/patterns of recursion behaviour? How does seasonal and climate variability influence long-term recursion behaviour and resource-use? Additionally, we could ask whether body size and metabolic requirements influence recursion by conducting a 
comparative study between large and small herbivores. While I was not surprised to find recursion by the Bornean elephant, the recursive behaviour of such a large, bulk feeding animal indicates that recursion is likely to be ubiquitous amongst mammalian herbivores, particularly selective feeders requiring higher quality food. Exploring recursion in both resource-rich and resource-poor areas would also allow further assessment of how recursion frequency and residency times vary according to habitat quality. For example, in poorer quality habitat does recursion occur very frequently, before plant recovery, and do short visits indicate inadequate resource availability?

The two temporal scales of short-term and long-term recursion identified in Chapter Four reflect elephant movement patterns among foraging sites. An investigation of movement was beyond the scope of the present study but further studies of recursion would benefit from investigations of the movement patterns across the landscape and recursion over different temporal scales. In order to thoroughly understand what governs the temporal scale of recursion, the next step would be to conduct analyses of spatial movement patterns throughout the animal home-range in conjunction with assessment of recursion to foraging sites. This topic was briefly addressed in Chapter Four (see also Appendix Three). The recursion patterns exhibited by the elephants could be tested by random or stratified random movement analysis using random-walk models, such as correlated random walks (McCulloch \& Cain, 1989). Alternatively long-term recursions are likely to occur as a result of lengthy, directed moves to clusters of foraging sites followed by short-term recursions within the clusters. In order to determine if this is true for elephants in LKWS, all foraging sites should be sampled in sequence rather than opportunistically throughout the elephant herd's range as they were for the present study. This approach would allow for analyses of movement between recursion sites and explain how movement patterns relate to long- and short-term recursions. 


\section{APPENDIX ONE \\ Distance across elephant herd when foraging}

\section{Introduction}

In order to determine the size of a foraging site and within what radius of the measurement point recursion was quantified for Chapter Four, the distance across the elephant herd while foraging was measured. A similar technique was used in a previous study of recursion by Bar-David et al., (2006) who established the area of a recursion site based on the spread of the Buffalo herd whilst feeding.

\section{Method}

Two adult female elephants from the two main herds in the LKWS population, each consisting of approximately 20-30 individuals, were randomly selected to attach a collar with a GPS transmitter in 2011. Positions were recorded at hourly intervals for 12-months from April 2011 to May 2012. We identified and visited sites used by elephants throughout their range in April and May 2011, the early to mid-dry season. To identify sites used by the elephant herds, the latest GPS position of each group was downloaded (www.awt.co.za) then the elephant herd tracked by following footprints, dung, feed sign and vocalisations until the herd was located.

Once an elephant group was sighted we positioned ourselves at a distance so as not to disturb them. The activity of the majority of elephants at the site at the time of observation was recorded as either foraging, resting or moving. Moving sites were those where the focal female and her herd were seen walking without stopping to feed. Resting sites were areas where the majority of elephants in the group were seen standing motionless, apart from ear and tail movement, or lying down and not feeding. Foraging sites were areas where the majority of the herd were seen collecting and ingesting food at the time of observation. The distance across the herd whilst foraging was measured on 86 occasions using a combination of direct observations, vocalisations of the herd and, after the herd had moved away, retracing the area used by the herd based on footprints and dung. This distance across the herd was then used to determine the approximate size of a foraging site. 


\section{Results \& Discussion}

The average spread of the elephant herds whilst foraging was $103.14 \pm 33.2 \mathrm{~m}$ (Figure 1). A foraging site was therefore determined as a $100 \mathrm{~m}$ radius surrounding the position where site measurements took place, which was at the centre of where the elephant herd were observed foraging (see Chapter Four).

\section{Average distance across elephant herd at measurement sites}

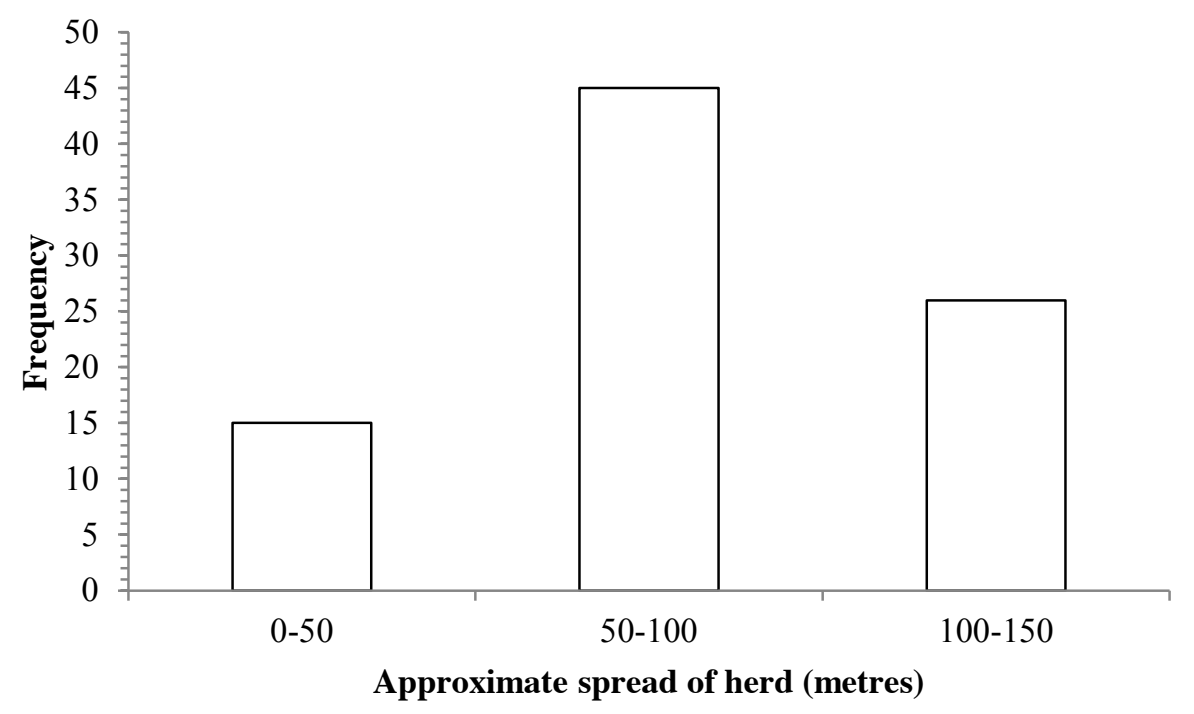

Figure 1. The approximate spread of the elephant herd was estimated from 86 measurement sites. Measurements were later grouped into 3 categories $(0-50 \mathrm{~m}, 50-100 \mathrm{~m}, 100-150 \mathrm{~m})$. 


\section{APPENDIX TWO \\ Comparison of data between two focal female elephants}

\section{Introduction}

Prior to analysing data for Chapter Four, a comparison between datasets for the two focal females was undertaken. In order to determine if recursion data needed to be analysed separately for the two herds, or pooled into one dataset, a comparison between the frequency of recursion events, the density of points at each site and the influence of site characteristics on recursion was compared between the two females.

\section{Method}

Data from the two collared female elephants was compared for significant difference between individuals (see Appendix One). A Generalised Linear Model was used where elephant 1 or 2 was the dependent variable and the independent variables were the number of foraging sites, number of recursion sites, density of points within sites, density of points within different habitat types, average distance of sites to water, average distance of sites to human habitation, average shade percentage of sites and average understorey cover percentage at sites (See Chapter Four Methods).

\section{Results \& Discussion}

No significant difference was found between the two focal females for the occurrence of recursion $\left(x^{2}=0.014, P=0.905\right)$, hours at site $\left(x^{2}=0.768, P=0.381\right)$, habitat type $\left(x^{2}=0.039\right.$, $P=0.844)$, distance to water $\left(x^{2}=0.410, P=0.522\right)$, distance to human disturbance $\left(x^{2}=\right.$ $0.125, P=0.724)$, understorey $\%\left(x^{2}=0.048, P=0.827\right)$. A significant difference was found in shade $\%\left(x^{2}=4.069, P=0.044\right.$, Figure 1$)$. 


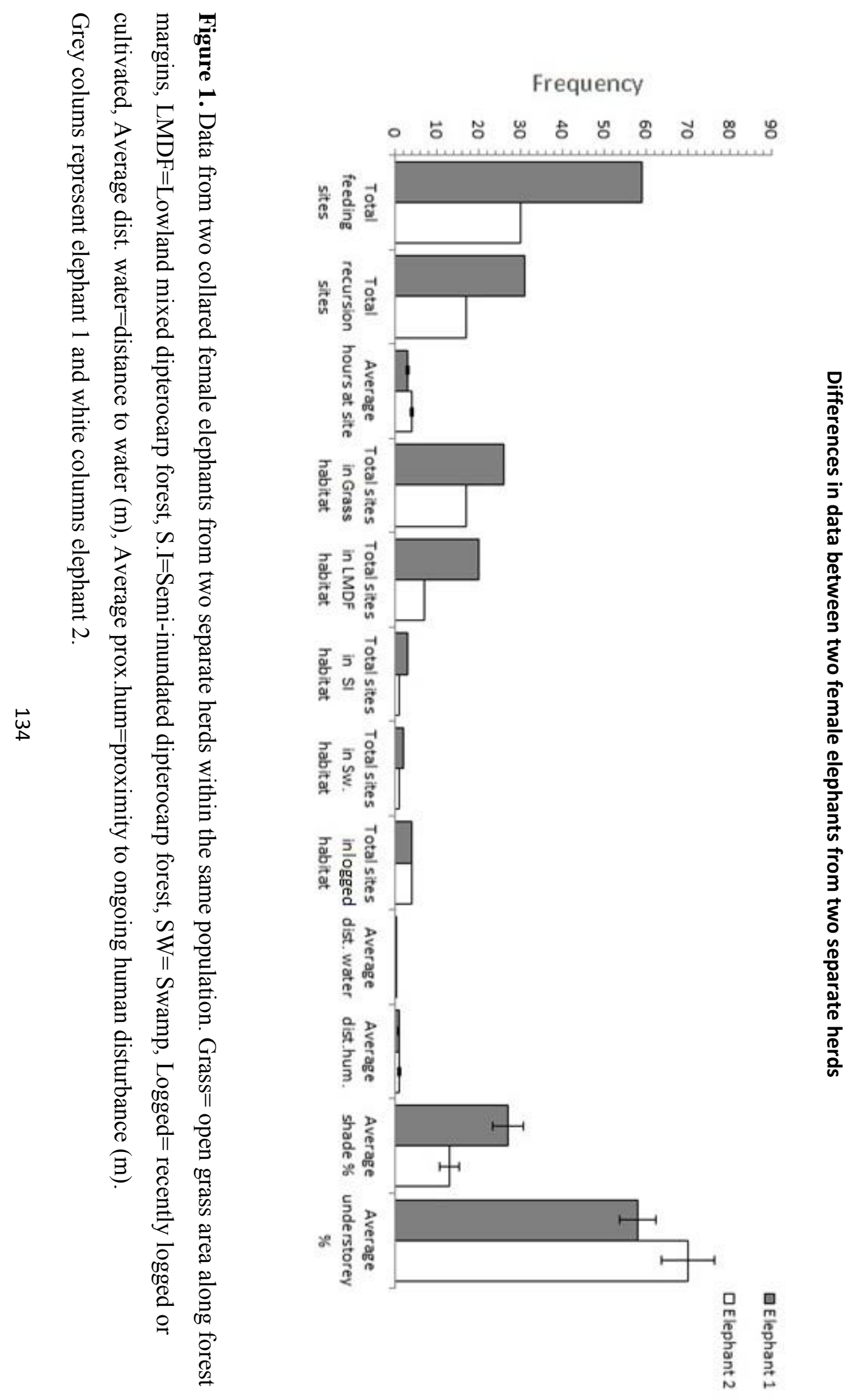




\section{APPENDIX THREE}

\section{Preliminary analyses of elephant movement in LKWS using a Hidden Markovian Model}

\section{Introduction}

Movement ecology proposes that animals should switch between movement modes as a complex function of internal state, landscape characteristics, motion capacity and navigational capacity (Nathan et al., 2008). Whether animals are directing their movement toward distant goals or following a correlated random walk (CRW) with no distinct goals, analysis of these movement paths can reveal changes in the rate of travel that correspond to changes in foraging behaviour (Johnson et al., 2002; Fauchald \& Tveraa, 2003; Brooks \& Harris, 2008). For example, if food is readily available, a random walk (RW) without complex search mechanisms is expected (Focardi \& Tinelli, 1996). This pattern might also be expressed as a CRW (Kareiva \&Shigesada, 1983; Bovet \& Benhamou, 1988), where movement is governed at a small spatial scale, with animals altering their movement paths according to local environmental stimuli.

Over short temporal scales the movement of an animal can be described by using mathematical models such as RW's and CRW's (Okubo, 1980; Turchin, 1998; Okubo \& Levin, 2001). However, over longer temporal scales models such as these do not often describe changes in patterns of movement associated with different behavioural states of the individual (Firle et al., 1998; Morales \& Ellner, 2002). According to Morales et al. (2004) one way to accommodate multiple behaviours is to develop different movement models for a number of states of movement (Grünbaum, 2000; Skalski \& Gilliam, 2003). An analysis, using Hidden Markovian Models (HMM) can identify multiple underlying behavioural states. RW's and CRW's are very simple special cases of HMM's. A HMM resembles a multi-state random walk. There are multiple behavioural states, and in each of these states the animal performs a particular type of random walk. For example, when in an intensive search state such as after encountering a habitat patch with abundant food, step lengths will be short, turns will be frequent and turning angles large. In contrast, extensive search states will be characterized by longer step lengths and small and infrequent turning angles (Zollner \& Lima, 1999; Langrock et al., 2012). 


\section{Method}

Observed movement data

From May 2011- May 2012 a local network (Celcom) recorded the hourly positions of a female elephant using a GSM satellite collar (downloaded from www.awt.co.za). Data supplied included the date, time, hourly GPS position, altitude, speed and movement direction.

The movement pathmetrics tool in the Geospatial Modelling Environment for ArcGIS (version 10) was used to calculate the step length 1 (hourly distance in metres), and turning angle $\alpha$ (the absolute angle between the previous step and the next step, with $0^{\circ}$ representing movement in the same direction as previoulsy) between 7884 data points.

\section{Hidden Markovian model using observed data}

A HMM is a time series model that comprises two components, an observable series and an underlying, non-observable state sequence. The observed variables relate to some quantification of movement, in this case the step lengths and turning angles of the observed data. The behavioural states cannot be observed (i.e. they are hidden) and are designed to account for over-dispersion and serial correlation in the observed series. In the present context of animal movement they can be interpreted as behavioural states of the observed animal (Langrock et al., 2012). This HMM allows movement patterns to change according to switches in the underlying behavioural state process. The HMM was conducted using $\mathrm{R}$ 2.12.1.

\section{Results \& Discussion}

In both the encamped and active states, the elephant performed a CRW, but the two CRW's were determined by different parameters and thus involved different movement dynamics. Figure 1 illustrates how black lines correspond to state 1, the state in which the elephant is not very active, in terms of movement throughout their range. Red lines correspond to state 2 (active) where the elephant on average takes much longer steps. The state that leads to higher steps, state 2, also involves a higher directional persistence than the other. Figure 2 illustrates movement points derived from the observed data. 
During both the encamped and active states the elephant movement followed a correlated random walk. However, more directional persistence and longer step lengths occurred during the active state and more sinuosity and shorter step lengths occurred during the encamped state, although turning angles were still quite directional in the latter. The movement pattern appears to follow long moves followed by short moves between clusters of points. This may reflect long movement to resource-rich areas followed by short moves within these areas.

How recursion can be incorporated into a HMM is yet to be fully understood. Also, how movement across the home-range and between foraging sites might influence recursion requires further investigation and ideally sampling of all feeding sites in sequence throughout the elephant range. 


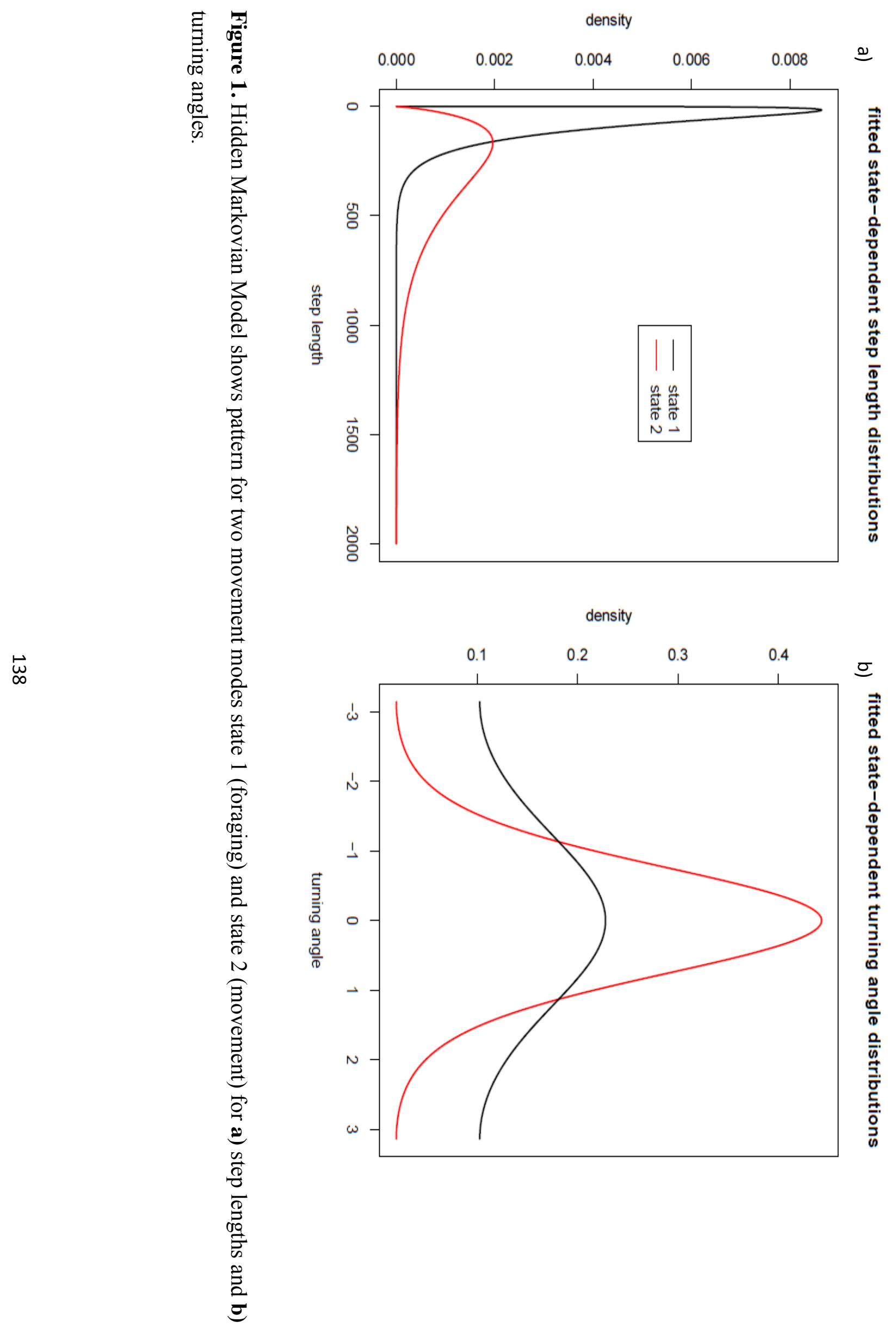




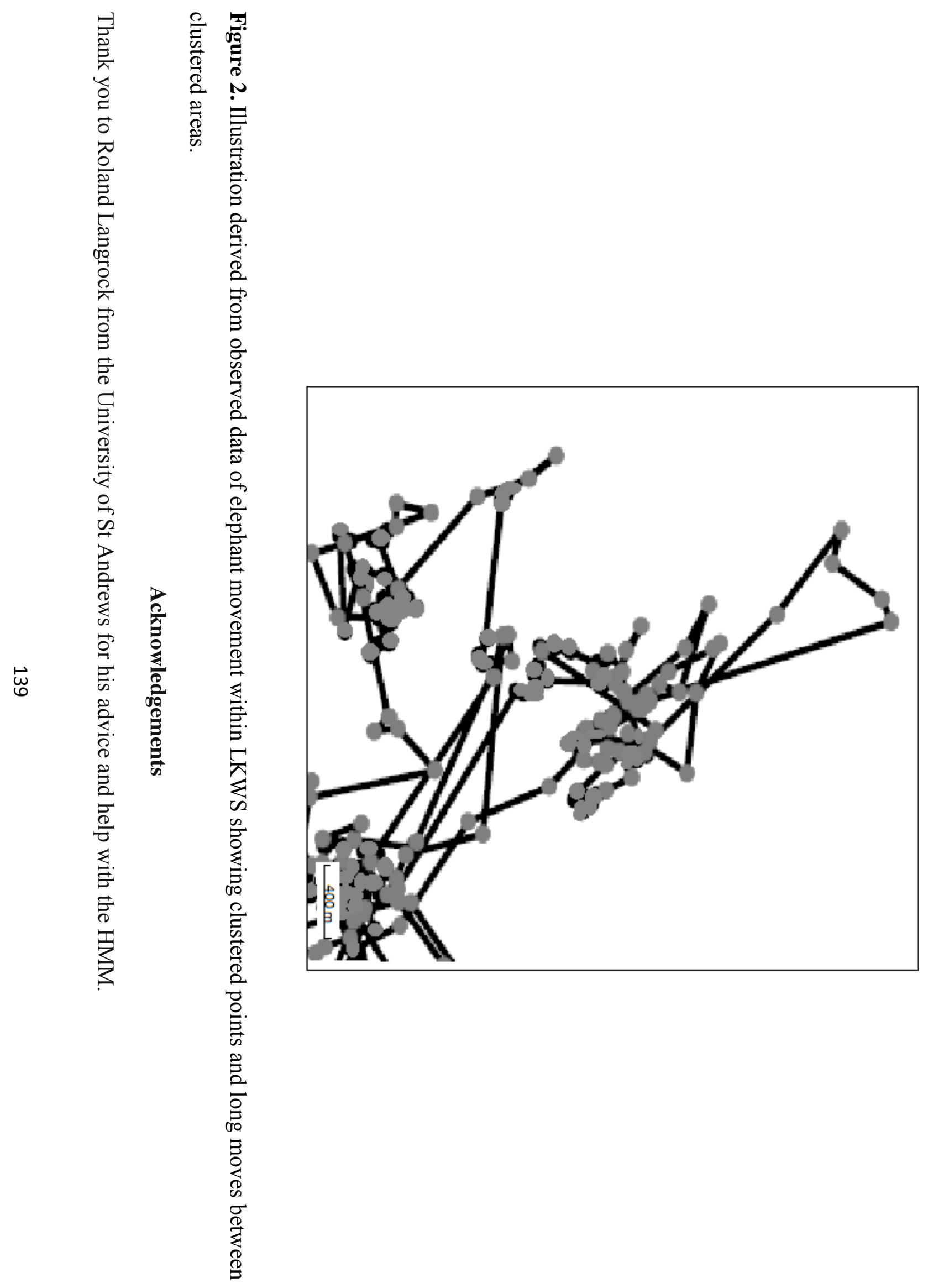




\title{
Foraging site recursion by forest elephants Elephas maximus borneensis
}

\author{
Megan ENGLISH ${ }^{1 *}$, Marc ANCRENAZ ${ }^{2}$, Graeme GILLESPIE ${ }^{3}$, \\ Benoit GOOSSENS ${ }^{4,5,6}$, Sen NATHAN ${ }^{6}$, Wayne LINKLATER ${ }^{1,7}$ \\ ${ }^{1}$ Centre for Biodiversity and Restoration Ecology, School of Biological Sciences, New Kirk Building, Kelburn Parade, Kelburn \\ Campus, Victoria University of Wellington, 6012, New Zealand \\ ${ }^{2}$ Hutan Kinabatangan Orangutan Conservation Project and Elephant Conservation Unit, Sukau, Kinabatangan, Sabah, Malaysia \\ ${ }^{3}$ Zoology Department, University of Melbourne, Parkville, Victoria, 3010, Australia \\ ${ }^{4}$ Danau Girang Field Centre, c/o Sabah Wildlife Department, Wisma Muis, 88100 Kota Kinabalu, Sabah, Malaysia \\ ${ }^{5}$ Organisms and Environment Division, School of Biosciences, Cardiff University, Sir Martin Evans Building, Museum Avenue, \\ Cardiff CF10 3AX, UK \\ ${ }^{6}$ Sabah Wildlife Department, Wisma Muis, 88100 Kota Kinabalu, Sabah, Malaysia \\ ${ }^{7}$ Centre for African Conservation Ecology, Nelson Mandela Metropolitan University, Port Elizabeth, South Africa
}

\begin{abstract}
Recursion by herbivores is the repeated use of the same site or plants. Recursion by wild animals is rarely investigated but may be ubiquitous. Optimal foraging theory predicts site recursion as a function of the quality of the site, extent of its last use, and time since its last use because these influence site resource status and recovery. We used GPS collars, behaviour and site sampling to investigate recursion to foraging sites for two elephant Elephas maximus borneensis herds in the Lower Kinabatangan Wildlife Sanctuary, Borneo, over a 12 month period. Recursion occurred to 48 out of 87 foraging sites and was most common within 48 hours or between 151-250 days, indicating two different types of recursion. Recursion was more likely to occur if the site had previously been occupied for longer. Moreover, the time spent at a site at recursion was the same as the time spent at the site on the first occasion. The number of days that had passed between the first visit and recursion was also positively correlated with how much time was spent at the site at recursion. Habitat type also influenced the intensity of site-use, with more time spent at recursion within riverine/open grass areas along forest margins compared to other habitat types. Recursion is a common behaviour used by the elephants and its pattern suggests it may be a foraging strategy for revisiting areas of greater value. The qualities of recursion sites might usefully be incorporated into landscape management strategies for elephant conservation in the area [Current Zoology 60 (4): 551-559, 2014].
\end{abstract}

Keywords Asian elephant, Recursion, Site re-use, Herbivory, Megaherbivore, Optimal foraging

Understanding how animals utilise and navigate a landscape is essential to comprehending species ecological and population processes (Stephens and Krebs, 1986; Turchin, 1991; Bartumeus et al., 2005). Feed quality and the spatial distribution of foraging sites may influence foraging strategies of herbivores and the way in which they utilise the landscape (Owen-Smith and Novellie, 1982; Belovsky, 1984; Pyke, 1984; Senft et al., 1987; Adler et al., 2001). Recognising foraging patterns across the landscape has benefits for species and habitat conservation. The spatial and temporal variation in species foraging behaviour and habitat utilisation may be incorporated into landscape management strategies and habitat assessment.

The return by animals to previously utilised foraging sites is known as recursion. Studies of recursion are few (Bar-David et al., 2009; Li et al., 2012; Riotte-Lambert et al., 2013). However, studies of animal behaviors that resemble recursion have been explored more extensively. Those studies used different terminology to recursion but have explored a similar process. Examples of foraging recursion include, the influence of spatial memory and resource relocation in bees (Kadmon, 1992; Williams and Thomson, 1998; Stout and Goulson, 2002; Menzel et al., 2005), nectar feeding birds returning to flowers (Gill, 1988; Burke and Fulham, 2003), sheep and cattle returning to grass patches (Bailey et al., 1989; Dumont and Petit, 1998) and primates returning to fruit trees (Garber, 1988; Garber and Jelinik, 2006; Erhart and Overdorff, 2008; Janson, 1998; Watts, 1998; Porter

Received April 12, 2014; accepted June 24, 2014.

* Corresponding author. E-mail: Megan.English@vuw.ac.nz

(C) 2014 Current Zoology 
and Garber, 2013; Janmaat et al., 2013).

Temporal patterns in site revisits have been related to resource recovery, for example in insects (Bell, 1990; Williams and Thomson, 1998) and birds (Motacilla sp.) (Davies and Houston, 1981). The predictability and order of repeated visits to previously utilised sites has been explored extensively in bumblebees (Bombus sp.) (Comba, 1999; Heinrich, 1976; Manning, 1956; Thomson, 1996; Thomson et al., 1982, 1987), euglossine bees (Euglossini spp.) (Janzen, 1971; Ackerman et al., 1982; Dressler, 1982), butterflies (Danaus sp.) (Gilbert, 1980), hummingbirds (Phaethornis spp.) (Gill, 1988; Garrison and Gass, 1999; Tiebout, 1991), wagtails (Motacilla sp.) (Davies and Houston, 1981), bats (Glossophaga sp., Pippistrellus sp.) (Lemke, 1984; Racey and Swift, 1985), and primates (Saguinus spp., Cebus sp., Gorilla sp.) (Garber, 1988; Janson, 1998; Watts, 1998). Additionally, the influence of resource renewal on site re-use has been explored using simulations of animal movement patterns (Possingham, 1989; Bar-David et al., 2009; Ohashi and Thomson, 2005). Nevertheless, only some studies have explored foraging site re-use in uncontrolled environments and these studies are largely limited to primates (Garber, 1988; Watts, 1998; Garber and Jetlinik, 2006; Erhart and Overdorff, 2008; Porter and Garber, 2013; Janmaat et al., 2013) and a few ungulates (Syncerus sp., Aepyceros sp.) (Bar-David et al., 2009; Riotte-Lambert et al., 2013).

Habitat quality and its importance to animals, or individual resource quality and its importance within foraging sites, might be best understood by measuring the amount of time animals spend at these sites and how frequently they return to them. Single point-in-time surveys, designed to identify selected resources or avoided habitat features, that do not take into account temporal variation in resource use might under- or over- estimate the relative importance of sites visited and their resource characteristics. If an animal repeatedly visits a site and spends more time at a site relative to others, this may help to identify high quality areas or more critical resources.

We investigated foraging site recursion by a wild population of Bornean elephants Elephas maximus borneensis and the foraging mechanisms that might explain recursion behavior. Our aims were to identify if recursion occurs, how often, and what environmental and biological variables may influence this behavior. In an optimally foraging herbivore, recursive site-use should be a function of the intensity of last use. Recursion should also relate to time since last use to allow for resource recovery after depletion (Ohashi and Thomson,
2005). The composition of the site should influence recursion because a herbivore will have specific resource requirements and site quality and recovery rates may vary with endogenous (for example, nutrient content) and exogenous (for example, climatic) influences. Recursion behavior may also, however, be an indicator of diminishing habitat of suitable quality or capacity because, as prime habitat becomes less available, recursion frequency should increase but time spent at sites decline. However, over time we would expect an optimally foraging herbivore to avoid unprofitable areas.

We expected elephants to spend more time at recursion sites compared to non-recursion sites if the former were higher quality, such as those sites containing preferred food plants like grasses and bamboos (English et al., 2014). Additionally, we expected elephants to return to sites at two temporal scales. Longer recursion times should occur in order for resources to sufficiently recover, especially where the site was used more extensively previously. Short-term recursions should occur as part of a site-sampling strategy (Owen-Smith, 2002). We also expected site characteristics such as habitat type, distance to water, distance to human habitation, shade and understorey cover to influence recursion and the intensity of site use. We expected more intensive use of sites within habitats containing preferred food plants of the Bornean elephant, such as within riverine and open grass areas along forest margins (English et al., 2014), sites closer to a water source (McKay, 1973; Sukumar, 1990), sites further from human habitation (Blake, 2002) and sites less shaded (Barnes et al., 1995; Powell, 1997) allowing for more understorey growth, in particular grasses and bamboos (McKay, 1973; Shoshani and Eisenberg, 1982).

\section{Materials and Methods}

\subsection{Study site}

The Lower Kinabatangan Wildlife Sanctuary (LKWS) is located in Eastern Sabah, Malaysia. The sanctuary is a lowland floodplain that comprises a matrix of habitat types in predominantly degraded forest ecosystems. The floodplain is characterized by a warm, wet and humid tropical climate. Mean monthly temperatures range between $21^{\circ} \mathrm{C}$ and $34^{\circ} \mathrm{C}$ (Ancrenaz et al., 2004). Flooding occurs mainly between November and March during the west monsoon. Rainfall averages $3000 \mathrm{~mm} \mathrm{y}^{-1}$ (Sooryanarayama, 1995). Soils are predominantly alluvial and derived from sedimentary deposits often rich in magnesium. In areas of freshwater swamps soils contain a high proportion of peat (Azmi, 1998). 
This study focused on the area between the villages of Abai and Batu Puteh (518'-N 542'-N, 117 ${ }^{\circ} 54^{\prime}$-E $\left.118^{\circ} 33^{\prime}-\mathrm{E}\right)$, which were the downriver and upriver limits of the LKWS elephant population's range. The study area contains 7 sections, each section referred to as a 'lot' (approximately $218 \mathrm{~km}^{2}$ ), including $89 \mathrm{~km}^{2}$ of protected forest reserves (Estes et al., 2012). The elephant herds utilised their whole range throughout the year including use of privately owned forests and cultivated land, particularly oil palm plantations that were adjacent to and between forested areas.

\subsection{Focal species}

The Bornean elephant, an endangered sub-species of Asian elephant Elephas maximus (IUCN, 2013) is found only in the eastern and central parts of Sabah (Alfred at al., 2010) as well as the extreme north of Indonesian Kalimantan. The main threats to Bornean elephants are changes in habitat particularly conversion of forest to oil palm plantations and resulting elevation in humanelephant conflict. Elephants in LKWS are mostly restricted to the linear fragments of forest along the $\mathrm{Ki}$ nabatangan River (Estes et al., 2012) (Fig. 1).

\subsection{Site location}

Two adult female elephants from separate herds, each consisting of approximately 20-30 individuals, were randomly selected to attach a collar with a GPS transmitter in 2011. Neither female was the matriarch to her herd which she belonged. Positions were recorded at hourly intervals for 12 months from April 2011 to May 2012. We identified and visited sites used by elephant throughout their range in April and May 2011, the early dry season. To identify sites used by the elephant herds, the latest GPS position recorded of each group on the days of observation was located and then fresh elephant sign, (footprints, dung, feed sign and vocalisations) was tracked until the focal female and her herd were located.

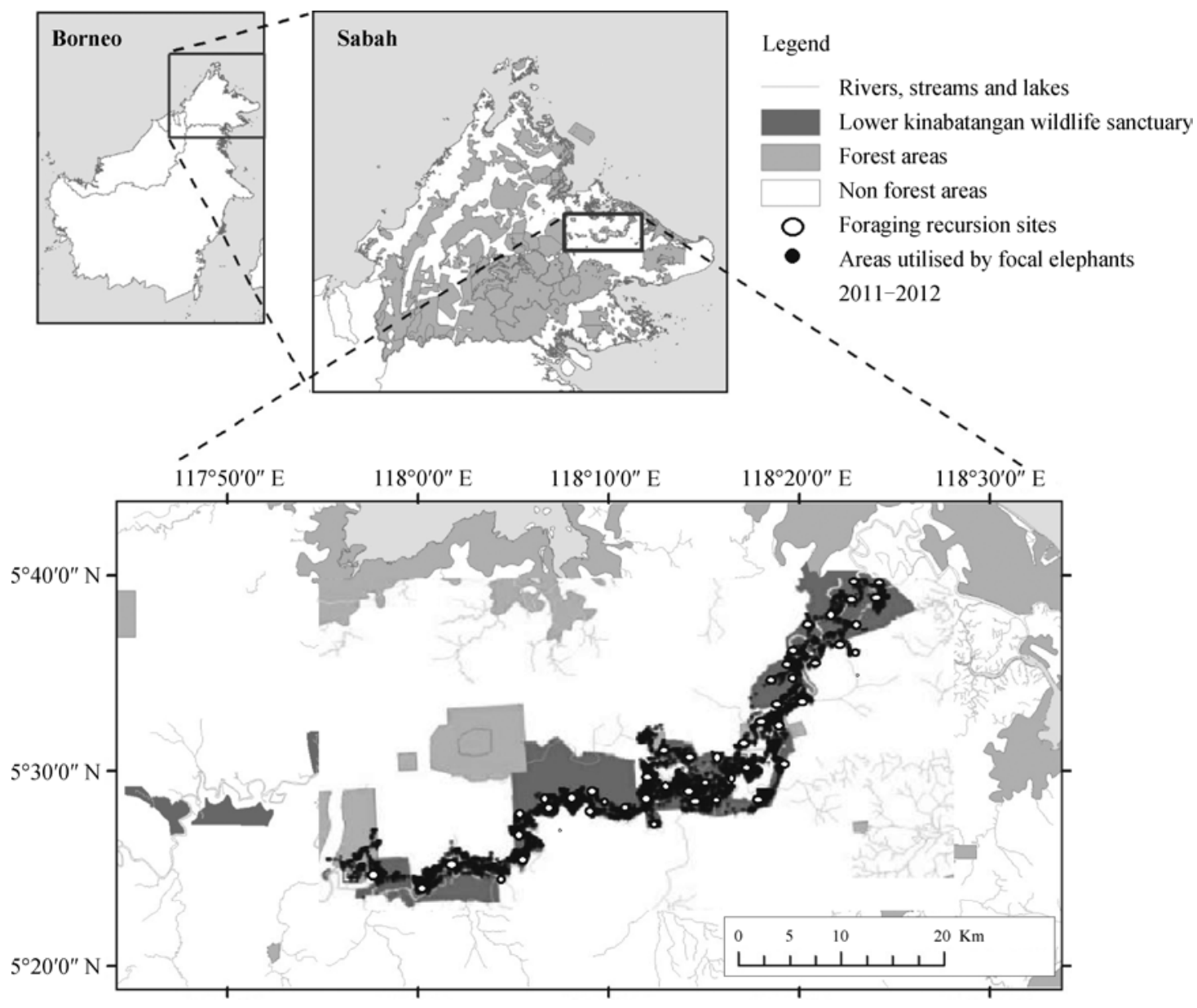

Fig. 1 Map of the Lower Kinabatangan Wildlife Sanctuary, Sabah (Adapted from www.cloudedleopard.org)

Black points illustrate the areas used by the two focal herds for 12 months from 1/04/11 to 1/04/2012. White points indicate the location of foraging sites that received recursion. 
Once an elephant group was sighted we positioned ourselves at a distance so as not to disturb them. The activity of the majority of elephants at the site at the time of observation was recorded as either foraging, resting or moving. Moving sites were those where the focal female and her herd were seen walking without stopping to feed. Resting sites were areas where the majority of elephants in the group were seen standing motionless, apart from ear and tail movement, or lying down and not feeding. Foraging sites, the focus of this study, were areas where the majority of the herd were seen collecting and ingesting food at the time of observation. Once the activity of the group was categorised we then counted the number of elephants in the group and then waited and allowed the group to move before sampling the site. These observations were carried out on the two separate groups.

\subsection{Site characteristics}

Foraging sites refer to areas in which we observed the herds during April-May 2011 (during the early to mid-dry season). These were the sites where recursion was measured. The habitat type of each site was categorised as one of riverine/open grass areas along forest margins, lowland dipterocarp forest, semi-inundated dipterocarp forest, swamp and highly disturbed/cultivated land according to previous classifications (Azmi, 1998; English unpub. data). Distance to the nearest water source (m), proximity to human habitation (m), shade (\%) and understorey cover (\%) were also recorded at each site. Distance to water and proximity to human habitation were measured using a Garmin 60csx Global Positioning System (GPS). Water sources included rivers, tributaries and ox-bow lakes but did not include swamps of stagnant, non-free flowing water. Proximity to human habitation was the distance in metres to areas with frequent human presence such as villages, dirt roads, bitumen roads and plantations. Shade percentage was measured using a spherical densiometer. This tool enabled calculation of the percentage of the forest canopy cover which was reflected onto a 24 cell grid convex mirror and measured near the centre of where the herd had been observed (see Lemmon 1956). Understorey cover was measured using a $2 \mathrm{~m}$ long PVC pipe marked every $2 \mathrm{~cm}$ along its length. The number of $2 \mathrm{~cm}$ marks covered by vegetation was counted from a distance of $5 \mathrm{~m}$ when the pipe was held horizontally 30 $\mathrm{cm}$ from the ground by an assistant. This was then converted to a percentage of understorey cover in an area resembling the understorey vegetation before elephant disturbance.

\subsection{Defining and measuring site recursion}

A site was defined as the area that covered $100 \mathrm{~m}$ radius surrounding each measurement point taken from the centre of the elephant herd. The density of hourly GPS recordings was used as a measure of the amount of time spent within a 100-m diameter of the measurement point at each foraging site and was quantified for comparison between recursion and non-recursion foraging sites. The number of individuals in each group was counted on one hundred separate occasions for each group and was between 20-30 individuals per group (Group 1= $25 \pm 0.24$ and Group 2= $26 \pm 0.26$ ).

Using the recorded GPS positions from the elephant collars uploaded to Google Earth (Google Inc. 2010) along with the locations of foraging sites, we counted the number of returns to each site within 12 months, since the date of direct observation. The time period between each return was quantified. GPS points with a Positional Dilution of Precision (PDOP) of $>6$ were removed from analyses due to lack of precision (Langley, 1999).

\subsection{Statistical analyses}

The data collected from both elephant groups was analysed using a $t$-test to compare differences in the frequency of recursion events, density of points at sites and the above mentioned habitat variables. The only variable that was significantly different between the two elephant groups was shade \% (English unpub.data). However this variable was later removed from further analyses and so the datasets were pooled (see below).

A Principal Components Analysis (PCA) reduced confounding effects of partial correlation between environmental variables (distance to water, proximity to human habitation, shade \% and understorey \%). The PC identified that understorey and shade were strongly associated with Eigenvalues $<1$ and were therefore removed from further analyses, thus reducing the number of covariates in the model to distance to water and proximity to ongoing human habitation.

A Generalised Linear Model (GLM) (SPSS version 18.0, 2009) was used to determine if foraging site characteristics including distance to water, proximity to human habitation and habitat type influenced the dependent variable of recursion $(1=$ recursion, $0=$ no recursion). The amount of time the herd spent at a foraging site at the first visit (when the herd was initially observed at the site) was also included to determine if this influenced whether the site was later returned to.

A further GLM was used to analyse foraging sites with recursion to determine if there was a relationship 
between the time spent at the site at the first visit, time (days) between visits, proximity to human habitation, distance to water, habitat type and the dependent variable - time spent at the site at recursion.

The number of foraging sites where elephants were seen feeding on grasses/bamboo, gingers, palms, lianas and woody species (Table 1) was quantified and compared between recursion and non-recursion sites using a one-way ANOVA test. Food plants selected were measured from direct observation of elephants feeding, with plants seen ingested by the elephants identified to species level and later categorised into the above growth forms.

A chi-square test was used to determine if recursion frequency varied over temporal scales or if variation occurred randomly with no significant influence of time.

\section{Results}

Eighty-seven foraging sites were measured. Seventy-five movement and 43 resting sites were also initially measured but not included in the analyses for this study, which focuses on recursion to foraging sites only.

Table 1 Plant species and their growth form selected by elephants at foraging sites in the Lower Kinabatangan Wildlife Sanctuary, Sabah

\begin{tabular}{|c|c|c|}
\hline Family & Genus & Plant form \\
\hline Poaceae & Dinochloa scabrida & Grass/Bamboo \\
\hline Poaceae & Pannicum sp. & Grass \\
\hline Poaceae & Phragmites karka & Grass \\
\hline Poaceae & Pennisetum sp. & Grass \\
\hline Zingiberaceae & Alpinia ligulata & Ginger \\
\hline Maranthaceae & Donax canniformis & Ginger \\
\hline Arecaceae & Licuala sp. & Palm \\
\hline Arecaceae & Calamus caesius & Palm \\
\hline Arecaceae & Arenga sp. & Palm \\
\hline Leguminosae & Spatholobus sp. & Liana \\
\hline Leguminosae & Fordia splendidissima & Liana \\
\hline Lophopyxidaceae & Lopophaxis mangai & Liana \\
\hline Dilleneaceae & Dillenia excelsa & Woody species \\
\hline Sterculiaceae & Pterospermum sp. & Woody species \\
\hline Euphorbiaceae & Mollotus sp. & Woody species \\
\hline Euphorbiaceae & Claoxylon sp. & Woody species \\
\hline Euphorbiaceae & Maccaranga sp. & Woody species \\
\hline Myrtaceae & Eugenia sp. & Woody species \\
\hline Moraceae & Ficus sp. & Woody species \\
\hline Guttiferae & Garcinia parvifolia & Woody species \\
\hline
\end{tabular}

Foraging sites were returned to by each herd over varying temporal scales ranging from within days, to weeks and months of the previous visit. Forty-eight foraging sites were returned to within 12 months. A large percentage of sites were returned to within 48 hours of the previous visit and the remaining sites were returned to over varying time-scales peaking again between 151250 days. The difference in recursion frequency through time, therefore, was not random (Chi-square, $d f=10, P$ $<0.01$. Fig. 2). Elephants spent more time at the first visit compared to the second visit if they returned within 48 hours to a site ( $t$-test, $n=11, P=0.00$ ).

We found a significant positive relationship between the hours at a site at the first visit and recursion $\left(\chi_{1}^{2}=\right.$ $10.68, P=0.001$, Fig. 3 ), with more time spent at sites that were returned to. No relationship was found between hours at the site on first visit and other biological or environmental site characteristics including distance to water, proximity to human habitation or habitat type.

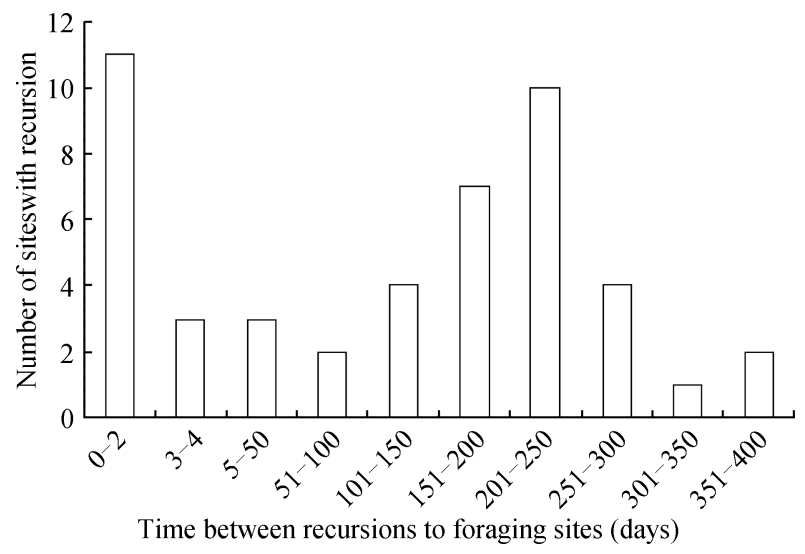

Fig. 2 Time period (days) between recursions by Bornean elephants in the Lower Kinabatangan Wildlife Sanctuary, Sabah, Malaysia over a 12 month period

A total of 48 foraging sites out of 87 were returned to.

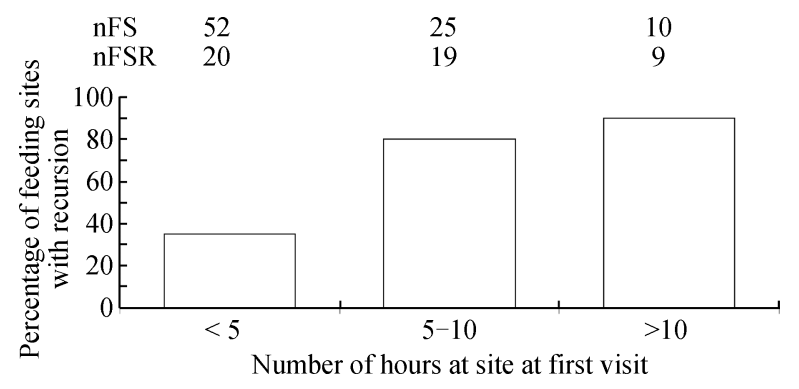

Fig. 3 Comparison between the time spent (hours) at recursion and non-recursion sites within the Lower Kinabatangan Wildlife Sanctuary, Sabah

The occurrence of recursion was higher to foraging sites where elephants had spent more time at the previous visit (nFS is the total number of feeding sites $=87, \mathrm{nFSR}$ is the total feeding sites with recursion $=48$ ). 
We also found a significant positive relationship between the number of days between visits (Fig. 4A), time spent at a site at the first visit (Fig. 4B) and habitat type (Fig. 4C) with the amount of time at a site at recursion (hours at first visit, Wald chi-square $=89.51, d f=1, P<$ 0.01 , days between returns, Wald $\chi^{2}=13.77, d f=1, P=$ 0.00 , habitat type, Wald $\chi^{2}=3.44, d f=1, P=0.043$ ).
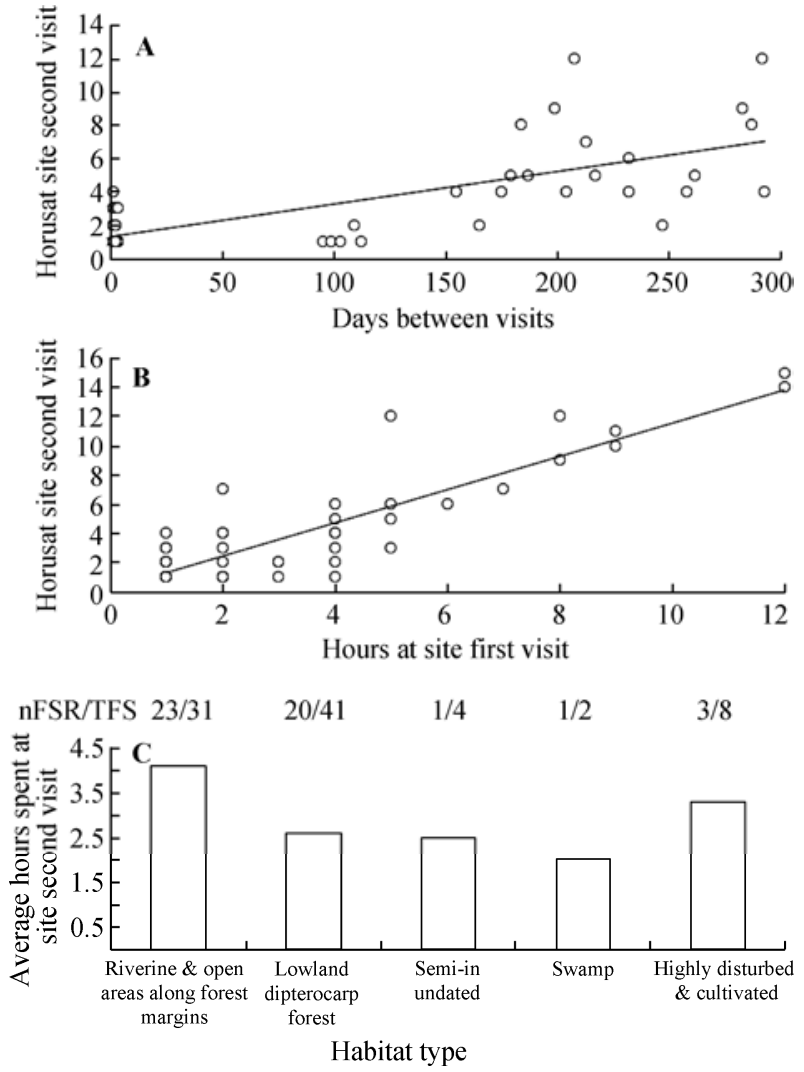

Fig. 4 Variables influencing time Bornean elephants within the Lower Kinabatangan Wildlife Sanctuary, Sabah spent at second visit to a site (i.e. $1^{\text {st }}$ recursion)

A) days between visits B) time spent at sites at first visit and $\mathbf{C}$ ) habitat. nFRS is the number of foraging sites that received recursion and nTFS refers to the total number of foraging sites within each habitat type.

From direct observations of the elephant herds foraging we found that there was a significant relationship between recursion and foraging sites where they were seen foraging on grasses and bamboos (ANOVA, $d f=$ 86, $F=14.041, P<0.001)$ and a significant relationship between foraging on lianas and sites that were not returned to within the 12 month study period (ANOVA $d f$ $=86, F=8.501, F=0.005$ ) (Fig. 5). Woody plants, palms and gingers had no significant influence on recursion $(P=0.095, P=0.157$ and $P=0.504$ respectively).

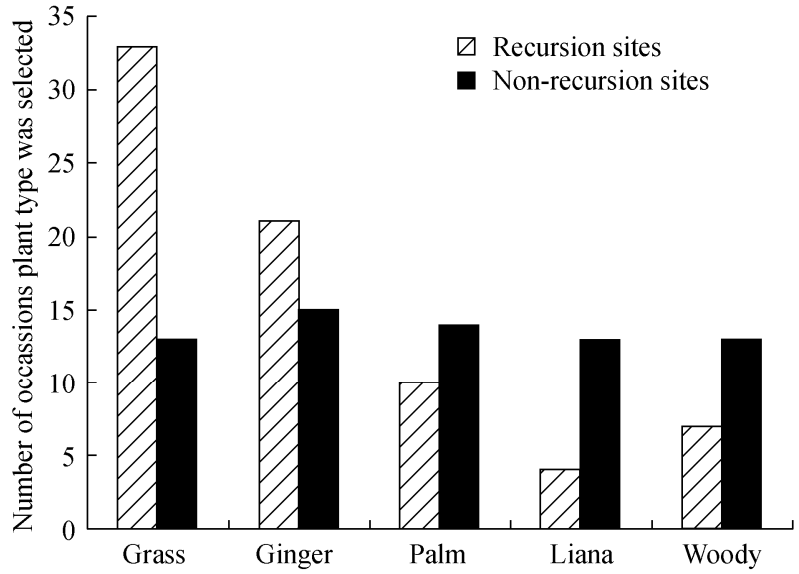

Fig. 5 Plant types selected for feeding by Bornean elephants within LKWS at recursion and non-recursion sites

\section{Discussion}

Recursion by elephants in the LKWS was common. It most commonly occurred during two time periods: within 48 hours of the previous visit and between 151250 days after the last visit, suggesting there are two different reasons for recursion.

We also found that the occurrence of recursion to foraging sites was influenced by the amount of time elephants had spent at a site previously, with recursions occurring more often to sites where they had spent more time in the past. A positive relationship was found between the number of days between the first visit and recursion, the number of hours spent at a site at the first visit, and the amount of time spent at a site at recursion. The habitat type of the site also influenced how much time was spent at a site during recursion. Additionally, elephants fed more on grasses and bamboos at recursion sites compared to sites they did not return to. Grasses and bamboos are most commonly found in the riverine and open grass habitats along forest margins (English et al., 2014) where elephants spent more time at recursion.

A relationship between the amount of time allocated to a particular area per visit and the resource quality of the area has previously been identified in large herbivore foraging behavior (Bailey et al., 1996). Our results suggest that, as more time was spent at recursion sites compared to non-recursion sites, then these sites, and sites with similar characteristics as recursion sites, are favourable to elephants and therefore should be considered high quality.

\subsection{Short -term recursions}

Recursions to a foraging site within 48 hours of the previous visit were expected to be a site-sampling stra- 
tegy where short duration visits were followed by longer duration visits, with the elephants returning to feed more extensively once the site quality was known relative to other sites in the area. We found the opposite, however. Elephants spent more time at sites at the first visit compared to the second visit (recursion) when they returned within 48 hours. This foraging strategy may be a way of reinforcing acceptance of a site over time (Owen-Smith, 2002). Alternatively, shorter second visits might be due to the elephants checking that all food within the site was exploited, resulting in less feeding time due to reduced food quality and insufficient regrowth of vegetation. Furthermore, induced responses in plants following herbivory, such as increased tannin content (Karban and Myers, 1989), could reduce the palatability of plants at sites recently visited.

\subsection{Long-term recursions}

Recursions after longer periods may be related to plant recovery rates (Bar-David et al., 2009) where herbivores return to exploit a feeding site once resources have sufficiently recovered. The more time elephants spent at sites at the first visit, the more days passed before they returned and the more time was spent at the site at recursion. This suggests that recursion occurring after 151-250 days is most likely related to resource recovery. To minimise energy expenditure and make optimal use of its home-range, a herbivore would benefit by monitoring vegetation depletion and renewal rates (McNaughton et al., 1997). This strategy would reduce the occurrence of returning to areas that had recently been exploited and facilitate the return after resource renewal.

Elephants may shift among sites in the same general region or move between a set of foraging areas. Recursions that occur after a longer time-period may occur after all sections have been exploited and enough time has passed for vegetation to regenerate in the areas previously visited (Bailey et al., 1996). This differs from recursions that occur within 48 hours where insufficient time has passed for resource regeneration. Long-term recursions to previously foraged areas, therefore, may be a beneficial foraging strategy for herbivores, enabling them to consume regrowing vegetation in a stage of high primary productivity and palatability (McNaughton, 1985; Gordon and Lindsay, 1990). Moreover, recursions may accelerate nutrient cycling in highly foraged sites (Gordon and Lindsay, 1990; McNaughton et al., 1997) and maintain them as nutrient hotspots (Winnie et al., 2008). Elephants may also select certain plants at a foraging site at specific times of the year.
This could explain why some sites received less recursions than others, for example where they had selected woody plants previously.

Both long- and short-term foraging strategies may be a form of associative learning, although in some species returning to a resource once it has been replenished is innate (Burke and Fulham, 2003). Innate foraging decisions associated with the spatial and temporal availability of resources may also occur in elephants. However, for such a highly social and long-lived species with large home-ranges, learned behavior within elephant herds is likely, especially considering their highly developed spatial and temporal memory (Hart et al., 2008). It is therefore probable that elephants remember areas containing their preferred food choices and return to them after sufficient time has passed for resources to replenish.

\subsection{Implications for elephant habitat management}

The identification and conservation of sites of recursion for elephants should be a priority in the design and management of reserves. For the LKWS population, riverine and open grass areas along forest margins appear to be key recursion sites and so these areas should be a conservation priority. Loss of access to recursion sites, or sites with similar characteristics, from either anthropogenic or natural disturbance may lead to increased human-elephant conflict due to resources being sought elsewhere, such as in oil palm plantations. Additionally, conservation practitioners and wildlife departments should establish protected corridors linking recursion sites to ensure accessibility for elephants.

The temporal pattern of site recursion described in this study is a reflection of elephant movement patterns amongst foraging sites. An investigation of movement was beyond the scope of the present study but further studies of recursion will benefit from investigations of the movement patterns that support recursion over different temporal scales. We observed recursion to occur over two time-scales: short- and long-term. Such a recursion could be supported by random or stratified random movement patterns, especially given spatial constraints of habitat boundaries, and so potentially modelled using random-walk models; for example, correlated random walks (McCulloch and Cain, 1989). Alternatively and much more likely, long-term recursions occur as a result of lengthy, directed moves to clusters of foraging sites followed by short-term recursions within the clusters. This type of movement would be expected to follow a Hidden Markovian Model (Langrock et al., 2012). The models that best explain movement pattern while also 
generating observed patterns of recursion remain to be found.

Aknowledgements Thank you to Victoria University of Wellington for supporting the study and providing a doctoral scholarship and a completion scholarship for Megan English, and to the Economic Planning Unit and Sabah Wildlife Department for permitting the research in the Lower Kinabatangan Wildlife Sanctuary. Thank you to Sulaiman Ismail from HUTAN Elephant Conservation Unit for helping with data collection and Danau Girang Field Centre and Nurzhafarina Othman for allowing access to the collared elephant data. Thank you to Andrew Hearn from the Bornean Clouded Leopard Programme for use of the map of LKWS. This project was supported by funds to Megan English and Dr Wayne Linklater from Zoos Victoria, Australia.

\section{References}

Ackerman JD, Mesler MR, Lu KL, Montalvo AM, 1982. Foodforaging behavior of male Euglossini (Hymenoptera, Apidae): Vagabonds or trapliners? Biotropica 14: 241-248.

Adler P, Raff D, Lauenroth W, 2001. The effect of grazing on the spatial heterogeneity of vegetation. Oecologia 128: 465-479.

Alfred R, Ahmad AH, 2010. Density and population estimation of the Bornean elephants Elephas maximus borneensis in Sabah. Online Journal of Biological Sciences, 10: 92-102.

Ancrenaz M, Calaque R, Lackman-Ancrenaz I, 2004. Orangutan nesting behaviour in disturbed forest of Sabah, Malaysia: Implications for nest consensus. International Journal of Primatology 25: 983-1000.

Azmi R, 1998. Natural Vegetation of the Kinabatangan Floodplain. Part 1: Background and Preliminary Checklist. Report. Kota Kinabalu, Sabah: WWF-Malaysia.

Bailey DW, Rittenhouse LR, Hart RH, Swift DM, Richards RW, 1989. Association of relative food availabilities and locations by cattle. Journal of Range Management 42: 480-482.

Bailey DW, Gross JE, Laca EA, Rittenhouse LR, Coughenour MB et al., 1996. Mechanisms that result in large herbivore grazing distribution patterns. Journal of Range Management 49: 386400.

Bar-David S, Bar-David I, Cross PC, Ryan SJ, Knechtel CU et al., 2009. Methods for assessing movement path recursion with application to African buffalo in South Africa. Ecology 90: 2467-2479.

Barnes RFW, Blom A, Alers MPT, Barnes KL, 1995. An estimate of the numbers of forest elephants in Gabon. Journal of Tropical Ecology 11: 27-37.

Bartumeus F, Da Luz MGE, Viswanathan GM, Catalan J, 2005. Animal search strategies: A quantitative random-walk analysis. Ecology 86: 3078-3087.

Blake S, 2002. The Ecology of Forest Elephant Distribution and Its Implications for Conservation. Ph.D. dissertation, Edinburgh, University of Edinburgh.

Bell W, 1990. Searching behavior patterns in insects. Annual Review Entomology 35: 447-467.

Belovsky GE, 1984. Herbivore optimal foraging: A comparative test of three models. American Naturalist 124: 97-115.
Burke D, Fulham BJ, 2003. An evolved spatial memory bias in a nectar-feeding bird? Animal Behaviour 66: 695-701.

Comba L, 1999. Patch use by bumblebees (Hymenoptera Apidae): Temperature, wind, flower density and traplining. Ethology Ecology and Evolution 11: 243-264.

Davies NB, Houston AI, 1981. Owners and satellites-the economics of territory defense in the pied wagtail Motacilla alba. Journal of Animal Ecology 50: 157-180.

Dressler RL, 1982. Biology of the orchid bees (Euglossini). Annual Review Ecological Systems 13:373-394.

Dumont B, Petit M, 1998. Spatial memory of sheep at pasture. Applied Animal Behaviour Science 60: 43-53.

English M, Gillespie G, Ancrenaz M, Ismail S, Goossens B et al., 2014. Plant selection and avoidance by the Bornean elephant Elephas maximus borneensis in tropical forest: Does plant recovery rate after herbivory influence food choices? Journal of Tropical Ecology 30: 371-379.

Erhart EM, Overdorff DJ, 2008. Spatial memory during foraging in prosimian primates: Propithecus edwardsi and Eulemur fulvus rufus. Folia Primatologica 79: 185-196.

Estes JG, Othman N, Ismail S, Ancrenaz M, Goosens B et al., 2012. Quantity and configuration of available elephant habitat and related conservation concerns in the Lower Kinabatangan floodplain of Sabah, Malaysia. PloS one 7: e44601.

Garber PA, 1988. Foraging decisions during nectar feeding by tamarin monkeys (Saguinus mystax and Saguinus fuscicollis, Callitrichidae, Primates) in Amazonian Peru. Biotropica 20: 100-106.

Garber PA, Jelinek PE, 2006. Travel patterns and spatial mapping in Nicaraguan mantled howler monkeys Alouatta palliata. In: Estrada A, Garber PA, Pavelka MSM, Luecke L ed. New Perspectives in the Study of Mesoamerican Primates. Springer US: 287-309.

Garrison JSE, Gass CL, 1999. Response of a traplining hummingbird to changes in nectar availability. Behavioural Ecology 10: 714-725.

Gilbert LE, 1980. Ecological consequences of a coevolved mutualism between butterflies and plants. In: Gilbert LE, Raven PH ed. Coevolution of Animals and Plants. Austin: University of Texas Press, 210-231.

Gill FB, 1988. Trapline foraging by hermit hummingbirds: Competition for an undefended, renewable resource. Ecology 69: 1933-1942.

Gordon I J, Lindsay WK, 1990. Could mammalian herbivores manage their resources? Oikos 59: 270-280.

Hart BL, Hart LA, Pinter-Wollman N, 2008. Large brains and cognition: Where do elephants fit in? Neuroscience and Biobehavioral Reviews 32: 86-98.

Heinrich B, 1976. The foraging specialisations of individual bumblebees. Ecological Monographs 46: 105-128.

IUCN, 2013. IUCN Red List of Threatened Species. Version 2013.2.

Janmaat KR, Ban SD, Boesch C, 2013. Taï chimpanzees use botanical skills to discover fruit: What we can learn from their mistakes. Animal cognition 16: 851-860.

Janson CH, 1998. Experimental evidence for spatial memory in foraging wild capuchin monkeys Cebus apella. Animal Behaviour 55: 1229-1243.

Janzen DH, 1971. Euglossine bees as long-distance pollinators of 
tropical plants. Science 171: 203-205.

Kadmon R, 1992. Dynamics of forager arrivals and nectar renewal in flowers of Anchusa stigosa. Oecologia 92: 552-555.

Karban R, Myers JH, 1989. Induced plant responses to herbivory. Annual Review of Ecology and Systematics 20: 331-348.

Langley RB, 1999. Dilution of precision. GPS World 10: 52-59.

Langrock R, King R, Matthiopoulos J, Thomas L, Fortin D et al., 2012. Flexible and practical modeling of animal telemetry data: Hidden Markov models and extensions. Ecology 93: 23362342.

Lemke TO, 1984. Foraging ecology of the long-nosed bat Glossophaga soricina, with respect to resource availability. Ecology 65: 538-548.

Lemmon PE, 1956. A spherical densiometer for estimating forest overstory density. Forest Science 2: 314-320.

Li Z, Han J, Ding B, Kays R, 2012. Mining periodic behaviors of object movements for animal and biological sustainability studies. Data Min. Knowl. Discovery 24: 355-386.

Manning A, 1956. Some aspects of the foraging behaviour of bumble-bees. Behaviour 9: 164-201.

McCulloch CE, Cain ML, 1989. Analyzing discrete movement data as a correlated random walk. Ecology 70: 383-388.

McKay GM, 1973. Behavior and ecology of the Asiatic elephant in southeastern Ceylon. Smithsonian Contributions to Zoology 125: 1-113.

McNaughton SJ, 1985. Ecology of a grazing ecosystem: The Serengeti. Ecological Monographs 55: 259-294.

McNaughton SJ, Banikwa FF, McNaughton MM, 1997. Promotion of the cycling of diet enhancing nutrients by African grazers. Science 278: 1798-1800.

Menzel R, Greggers U, Smith A, Berger S, Brandt R et al., 2005. Honey bees navigate according to a map-like spatial memory. Proceedings of the National Academy of Sciences of the United States of America 102: 3040-3045.

Ohashi K, Thomson JD, 2005. Efficient harvesting of renewing resources. Behavioral Ecology 16: 592-605.

Owen-Smith N, Novellie P, 1982. What should a clever ungulate eat? American Naturalist 119: 151-178.

Owen-Smith RN, 2002. Adaptive Herbivore Ecology: From Resources to Populations in Variable Environments. Cambridge: Cambridge University Press.

Porter LM, Garber PA, 2013. Foraging and spatial memory in wild Weddell's saddleback tamarins Saguinus fuscicollis weddelli when moving between distant and out-of-sight goals. International Journal of Primatology 34: 30-48.

Powell JA, 1997. The Ecology of Forest Elephants Loxodonta africana cyclotis in Banyang-Mbo and Korup Forests, Cameroon with Particular Reference to Their Role as Seed Dispersal Agents. Doctoral dissertation, University of Cambridge.

Possingham HP, 1989. The distribution and abundance of resources encountered by a forager. American Naturalist 133: 42-60.
Pyke GH, 1984. Optimal foraging theory: A critical review. Annual Review of Ecological Systems 15: 523-575.

Racey PA, Swift SM, 1985. Feeding ecology of Pipistrellus pipistrellus (Chiroptera, Vespertilionidae) during pregnancy and lactation. 1. Foraging behavior. Journal Animal Ecology 54: 205-215.

Riotte-Lambert L, Benhamou S, Chamaille-Jammes S, 2013. Periodicity analysis of movement recursions. Journal of Theoretical Biology 317: 238-243.

Senft RL, Coughenour MB, Bailey DW, Rittenhouse LR, Sala OE et al., 1987. Large herbivore foraging and ecological hierarchies. BioScience 37: 789-795.

Shoshani J, Eisenberg JF, 1982. Elephas maximus. Mammalian Species 182: 1-8.

Sooryanarayama S, 1995. Floods in Malaysia: Patterns and implications. Malaysian Journal of Tropical Ecology 26: 35-46.

SPSS, 2009. PASW Statistics for Windows, Version 18.0. Chicago: SPSS Inc.

Stephens DW, Krebs JR, 1986. Foraging Theory. Princeton: Princeton University Press.

Stout JC, Goulson D, 2002. The influence of nectar secretion rates on the responses of bumblebees (Bombus spp.) to previously visited flowers. Behavioral Ecology and Sociobiology 52: 239246.

Sukumar R, 1990. Ecology of the Asian elephant in southern India. II. Feeding habits and crop raiding patterns. Journal of Tropical Ecology 6: 33-53.

Thomson JD, Maddison WP, Plowright RC, 1982. Behavior of bumble bee pollinators of Aralia hispida Vent (Araliaceae). Oecologia 54: 326-336.

Thomson JD, Peterson SC, Harder LD, 1987. Response of traplining bumble bees to competition experiments: Shifts in feeding location and efficiency. Oecologia 71: 295-300.

Thomson JD, 1996. Trapline foraging by bumblebees: I. Persistence of flight-path geometry. Behavioural Ecology 7: 158-164.

Tiebout HM III, 1991. Daytime energy management by tropical hummingbirds: Responses to foraging constraint. Ecology 72: 839-851.

Turchin P, 1991. Translating foraging movements in heterogeneous environments into the spatial distribution of foragers. Ecology 72: $1253-1266$.

Watts DP, 1998. Long-term habitat use by mountain gorillas Gorilla gorilla beringei. 2 . Reuse of foraging areas in relation to resource abundance, quality, and depletion. Int. J. Primatol. 19: 681-702.

Williams NM, Thomson JD, 1998. Trapline foraging by bumble bees: III. Temporal patterns of visitation and foraging success at single plants. Behavioural Ecology 9: 612-621.

Winnie JA, Cross P, Getz W, 2008. Habitat quality and heterogeneity influence distribution and behaviour in African buffalo Syncerus caffer. Ecology 89: 1457-1468. 
Journal of Tropical Ecology

http://journals.cambridge.org/TRO

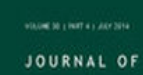

Additional services for Journal of Tropical Ecology:

Email alerts: $\underline{\text { Click here }}$

Subscriptions: $\underline{\text { Click here }}$

Commercial reprints: $\underline{\text { Click here }}$

Terms of use : $\underline{\text { Click here }}$

\section{Plant selection and avoidance by the Bornean elephant (Elephas maximus borneensis) in tropical forest: does plant recovery rate after herbivory influence food choices?}

Megan English, Graeme Gillespie, Marc Ancrenaz, Sulaiman Ismail, Benoit Goossens, Sen Nathan and Wayne Linklater

Journal of Tropical Ecology / Volume 30 / Issue 04 / July 2014, pp 371 - 379

DOI: 10.1017/S0266467414000157, Published online: 24 April 2014

Link to this article: http://journals.cambridge.org/abstract S0266467414000157

How to cite this article:

Megan English, Graeme Gillespie, Marc Ancrenaz, Sulaiman Ismail, Benoit Goossens, Sen Nathan and Wayne Linklater (2014). Plant selection and avoidance by the Bornean elephant (Elephas maximus borneensis) in tropical forest: does plant recovery rate after herbivory influence food choices? . Journal of Tropical Ecology, 30, pp 371-379 doi:10.1017/ S0266467414000157

Request Permissions : $\underline{\text { Click here }}$ 


\title{
Plant selection and avoidance by the Bornean elephant (Elephas maximus borneensis) in tropical forest: does plant recovery rate after herbivory influence food choices?
}

\author{
Megan English*,1, Graeme Gillespie†, Marc Ancrenazł, Sulaiman Ismailł, Benoit Goossens§;\#,**, \\ Sen Nathan** and Wayne Linklater*, $\dagger$
}

\author{
* Centre for Biodiversity and Restoration Ecology, School of Biological Sciences, New Kirk Building, Kelburn Parade, Kelburn Campus, Victoria University of Wellington, \\ New Zealand, 6012 \\ $†$ Zoology Department, University of Melbourne, Parkville, Victoria, 3010, Australia \\ $\ddagger$ HUTAN Elephant Conservation Unit and Kinabatangan Orangutan Conservation Project, Sukau, Kinabatangan, Sabah, Malaysia \\ $\S$ Danau Girang Field Centre, c/o Sabah Wildlife Department, Wisma Muis, 88100 Kota Kinabalu, Sabah, Malaysia \\ \# Organisms and Environment Division, School of Biosciences, Cardiff University, Sir Martin Evans Building, Museum Avenue, Cardiff CF10 3AX, UK \\ ** Sabah Wildlife Department, Wisma Muis, 88100 Kota Kinabalu, Sabah, Malaysia \\ i† Centre for African Conservation Ecology, Nelson Mandela Metropolitan University, Port Elizabeth, South Africa \\ (Received 29 October 2013; revised 17 March 2014; accepted 18 March 2014; first published online 24 April 2014)
}

\begin{abstract}
The plant vigour hypothesis proposes that herbivores should favour feeding on more vigorously growing plants or plant modules. Similarly, we would expect herbivores to favour plants that regrow vigorously after herbivory. Larger animals, like elephants, may also select plant species relative to their availability and prefer species with larger growth forms in order to meet their intake requirements. The food preferences of the Bornean elephant (Elephas maximus borneensis) in the Lower Kinabatangan Wildlife Sanctuary, Sabah, Malaysia, were investigated along 12 transects in areas where elephants were recently sighted feeding. One hundred and eighty-two plants were eaten and 185 plants were measured for species availability along transects. Species vigour was determined by the monthly regrowth in new shoot length after elephant feeding and the number of new shoots produced on each plant. Measurements were carried out on each plant for 9 mo or until the new shoot was eaten. Plant sizes were determined from their basal diameter. The Bornean elephant did not prefer more vigorous species or species with larger growth forms. New shoots did not grow longer on preferred than avoided species. Additionally, unlike other elephants that live in a forest environment, the Bornean elephant preferred species from the Poaceae (specifically Phragmites karka and Dinochloa scabrida) over other plant types including gingers, palms, lianas and woody trees.
\end{abstract}

Key Words: Borneo, Bornean elephant, feed preference, large herbivore, plant size, plant vigour

\section{INTRODUCTION}

The plant vigour hypothesis (PVH) proposes that herbivores prefer feeding on plants that grow more vigorously (Price 1991). A plant's 'vigour' refers to its comparative growth rate. The $\mathrm{PVH}$ has been supported in studies on insects (Baker 1972, Craighead 1950, Furniss \& Carolin 1977, Keen 1952, Price et al. 1987) and vertebrates (Bergström \& Hjeljord 1987, Danell et al. 1985). Vigorous plants should also grow faster after herbivory to compensate for the damage (Coley \& Aide 1990, Coley et al. 1985). More vigorous plants should

\footnotetext{
${ }^{1}$ Corresponding author. Email: Megan.English@vuw.ac.nz
}

have higher nutrient concentrations and less vigorous plants more chemical defences (Price 1991) and this may be the basis of herbivore foraging decisions.

Herbivore size may influence foraging decisions with respect to plant vigour. Larger herbivores, like elephant, are less selective of plant modules. Rather than responding only to plant chemical or structural properties, a larger herbivore may also respond to plant size (Vivas et al. 1991, Wilson \& Kerley 2003). Makhabu et al. (2006) found no relationship between the vigour of browse species and feeding preferences of the African elephant (Loxodonta africana). They suggested that the elephant selects whole plants, rather than just the new growth. The influence of plant vigour on food selection by Asian elephant species in a rain-forest environment has not yet been investigated. 
Elephant species should be less selective than other herbivores. Lower metabolic requirements, larger gut volume and food retention time, mean larger herbivores survive on lower-quality food (Bell 1971, Demment \& van Soest 1985, Jarman 1974, Shrader et al. 2012). They can trade food quality for quantity by consuming an abundant, low-value resource instead of searching for less common, higher-value forage (Demment \& van Soest 1985, du Toit \& Owen-Smith 1989). For this reason we propose that the $\mathrm{PVH}$ is unlikely to explain Asian elephant food choices, but that plants with larger growth forms will be preferred.

Plants have been found to respond both positively and negatively to herbivory. A plant's regrowth rate may increase or, if too much plant material has been taken, decrease because, for example, plant resources are invested instead in the production of antiherbivore defences (McNaughton 1983). We investigated whether plant size or regrowth vigour after herbivory influence Bornean elephant (Elephas maximus borneensis) preferences.

Herbivore food preferences are reflected by the vegetation chosen in proportion to its availability (Johnson 1980). Selection-availability or avoidance ratios reveal if animals fed randomly or selectively. Where browse is plentiful it dominates the diet of some forest elephant species (Blake 2002, Chen et al. 2006, Pradhan et al. 2008, Sukumar \& Ramesh 1992). But grasses were the major component of the diet of forest elephants throughout the year in Cameroon (Tchamba \& Seme 1993) and Olivier (1978) found the Malaysian elephant to avoid feeding on woody trees and prefer palms, herbs and grasses. A seasonal deterioration in grass quality has been used to explain a preference for browse by the Asian elephant (Pradhan et al. 2008, Sukumar 1990).

Based on the literature, we hypothesized that Bornean forest elephant diet would be dominated by species such as palms, gingers and woody trees rather than plants in the Poaceae, because the habitat types in which these are found are less common in this tropical forest landscape (English, unpubl. data). We expect elephant, therefore, to select plants proportional to their availability and especially the larger plants that provide abundant biomass. Due to an ability to feed on lower-quality food, because of its body size, we do not expect plant vigour to influence Bornean elephant food preferences.

\section{METHODS}

\section{Study site}

The Lower Kinabatangan Wildlife Sanctuary (LKWS) is located in South-Eastern Sabah, Malaysia. The sanctuary is a lowland floodplain that comprises a matrix of habitat types of degraded to highly degraded forest ecosystems. The floodplain is characterized by a warm, wet and humid tropical climate. Temperature variation is diurnal rather than seasonal and mean monthly temperatures range between $21^{\circ} \mathrm{C}$ and $34^{\circ} \mathrm{C}$ (Ancrenaz et al. 2004). Floods mainly occur between November and March during the west monsoon with rainfall averaging $3000 \mathrm{~mm}$ $\mathrm{y}^{-1}$ (Sooryanarayama 1995). Soils are predominantly alluvial, derived from sedimentary deposits often rich in magnesium and, in areas of freshwater swamp, soils often contain a high proportion of peat (Azmi unpubl. data).

This study focused on the area between the villages of Abai and Batu Puteh $\left(5^{\circ} 18^{\prime}-5^{\circ} 42^{\prime} \mathrm{N}, 117^{\circ} 54^{\prime}-\right.$ $118^{\circ} 33^{\prime} \mathrm{E}$ ), which were the downriver and upriver limits of the LKWS elephant population's range (Figure 1). The study area contains lots 1-7 (approximately $218 \mathrm{~km}^{2}$ ) of the LKWS including $89 \mathrm{~km}^{2}$ of protected forest reserves (Estes et al. 2012). 'Lots' represent the different sections of the sanctuary. Elephants also used the privately owned forests and cultivated land, such as oil palm plantations that were adjacent to and between forested areas.

\section{Focal species}

The Bornean elephant, an endangered subspecies of the Asian elephant (Elephas maximus), is found only in the eastern and central parts of Sabah (Alfred \& Ahmad 2010) as well as the extreme north of Indonesian Kalimantan. The main threat to the Bornean elephant population is the change in habitat from forest to agriculture, mainly oil palm plantations, and the resulting human-elephant conflicts. Elephant in LKWS are restricted to the linear fragments of forest along the Kinabatangan River (Estes et al. 2012).

\section{Vegetation sampling}

Sampling sites were selected opportunistically throughout the elephant's home-range. These sites represented the elephant range used by the herds in March-June 2011, which is early dry season (Figure 1). We searched sections of the sanctuary from the river and tracks for elephants and recent elephant signs. Signs included fresh dung, urine, fresh footprints and recently browsed plants.

We established 50-m transects at places where elephants were feeding. The transects were at least 300 $\mathrm{m}$ apart to minimize site autocorrelation. One transect was established per day. We tracked fresh elephant signs including footprints, dung and signs of feeding to establish the transect along the group feeding path. All plants showing signs of elephant feeding within $2 \mathrm{~m}$ either side of transects were marked and labelled with the date and a reference number. Samples of all plant species 


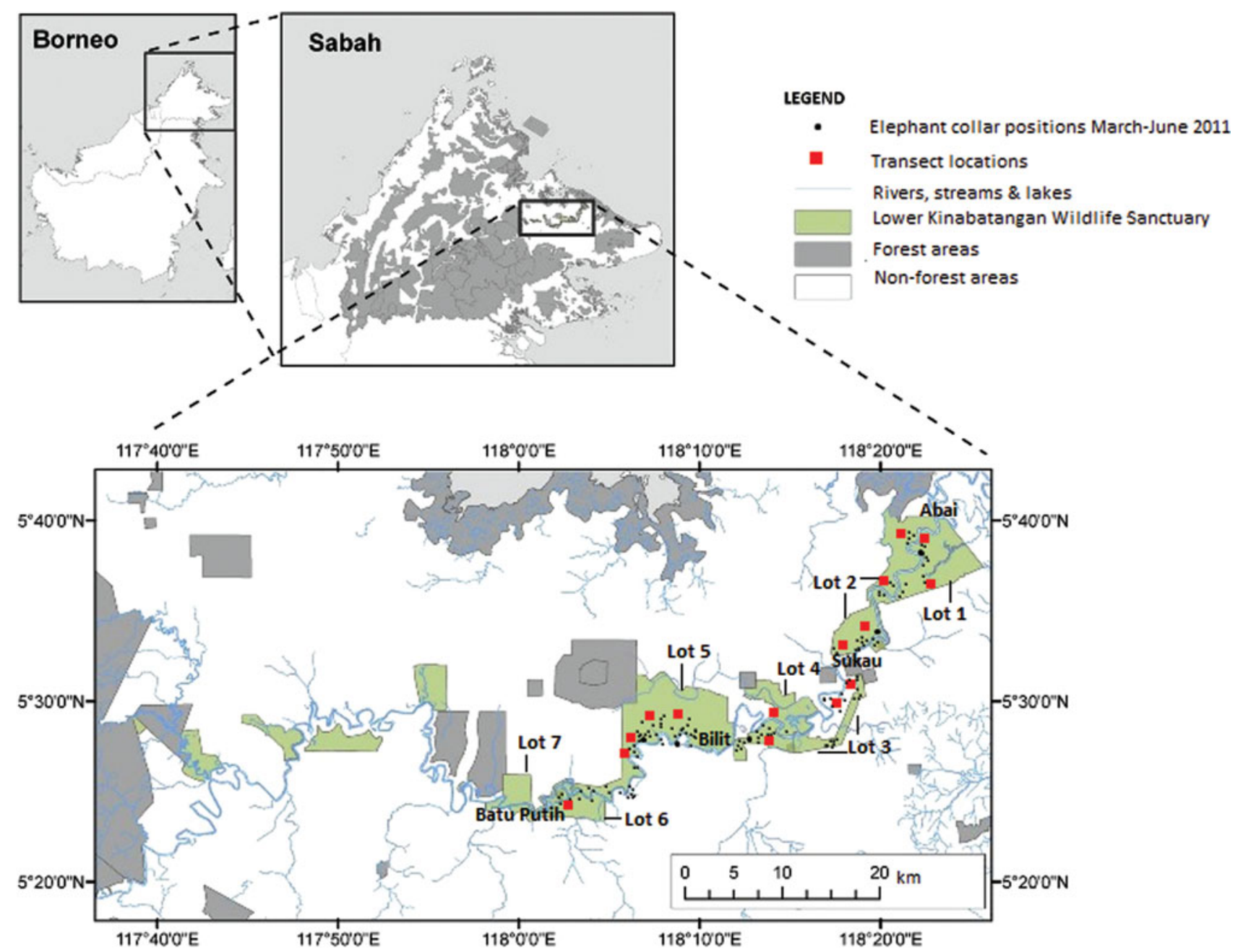

Figure 1. The Lower Kinabatangan Wildlife Sanctuary, Sabah, Malaysia (adapted from Clouded Leopard Project, Sabah, www.cloudedleopard.org).

were collected for identification at the Sabah Forestry Department Herbarium (SAN), Sandakan.

The height and basal diameter of the stem showing the feed signs were measured to establish the size and relative age of the plants selected by the elephants. Measures of availability were taken at 5-m intervals along the transect, where the species closest to the transect was recorded. Transects and marked plants were revisited and regrowth measured each month from April 2011 to December 2011. If the plant died or had been rebrowsed by elephant or other herbivores, thus preventing measurements of regrowth, this was also recorded.

Selected plants had diverse growth forms and responded to herbivore feeding by recovering in different ways. Monthly regrowth measurements were taken on a selected new shoot closest to the node nearest the feed sign, or from the plant base, depending on how the plant recovered. Only recovery visible above the ground and within a $30-\mathrm{cm}$ radius of the focal plant was measured. Measurements included new shoot growth in length, basal diameter of the new shoot and a count of the number of new shoots produced each month.

\section{Data analysis}

Plant species preference. Plant species preference was calculated using the relative availability (RA) of each species compared with their relative use (RU) by the Bornean elephant. We focused only on frequently encountered species by limiting the preference analysis to those species for which more than five individual plants were sampled. Species and sample sizes included in analysis are listed in Table 1.

$$
R A=\frac{N a}{T a}
$$


Table 1. Plant species selected by Bornean elephant and available along transects within the Lower Kinabatangan Wildlife Sanctuary, Sabah, Malaysia. Plant species are included in analyses for Figures 2 and 3 and were identified by Sabah Forestry Department herbarium (SAN), Sandakan, Sabah.

\begin{tabular}{|c|c|c|c|c|}
\hline $\begin{array}{l}\text { Plant group } \\
\text { (Figure 2) }\end{array}$ & Family & Species & $\begin{array}{c}\text { Total sample } \\
\text { size }\end{array}$ & $\begin{array}{c}\text { Code } \\
\text { (Figure 3) }\end{array}$ \\
\hline Grass & Poaceae & Phragmites karka (Retz.) Steud. & 91 & - \\
\hline Bamboo & Poaceae & Dinochloa scabrida S. Dransf. & 25 & - \\
\hline Ginger & Zingiberaceae & Alpinia ligulata K. Schum. & 49 & G \\
\hline Ginger & Costaceae & Costus speciosus J. Koenig & 12 & A \\
\hline Ginger & Marantaceae & Donax canniformis K. Schum. & 76 & $\mathrm{~F}$ \\
\hline Palm & Arecaceae & Licuala sp. & 11 & $\mathrm{~B}$ \\
\hline Palm & Arecaceae & Arenga sp. & 9 & $\mathrm{H}$ \\
\hline Palm & Arecaceae & Daemonorops sp. & 7 & I \\
\hline Liana & Leguminosae & Fordia splendidissima (Blume ex Miq.) J.R.M Buijsen & 12 & $\mathrm{~J}$ \\
\hline Woody & Melastomataceae & Memecylon sp. & 14 & $\mathrm{~K}$ \\
\hline Woody & Dilleniaceae & Dillenia excelsa (Jack) Gilg. & 11 & $\mathrm{~L}$ \\
\hline Woody & Rubiaceae & Gardenia elata Ridl. & 9 & M \\
\hline Woody & Myrtaceae & Syzygium sp. & 10 & $\mathrm{~N}$ \\
\hline Woody & Phyllanthaceae & Bridelia stipularis Blume & 9 & $\mathrm{O}$ \\
\hline Woody & Euphorbiaceae & Mallotus muticus (Muell. Arg.) Airy Shaw & 9 & $\mathrm{P}$ \\
\hline Woody & Sapindaceae & Lepisanthes fruticosa (Roxb.) Leenh. & 10 & $\mathrm{D}$ \\
\hline Woody & Guttiferae & Garcinia parvifolia (Miq.) Miq. & 8 & $\mathrm{C}$ \\
\hline Woody & Alangiaceae & Alangium javanicum (Bl.) Wang. & 6 & $\mathrm{E}$ \\
\hline
\end{tabular}

$\mathrm{Na}$ is the number of available plants of a given species and $\mathrm{Ta}$ is the number of available plants across all species (185).

$$
R U=\frac{N u}{T u}
$$

$\mathrm{Nu}$ is the number of times a species was selected. Tu is the total number of plants selected for feeding across all species (182).

$$
\text { Preference ratio }=\frac{R U}{R A}
$$

Species with a preference ratio $>1$ were selected and those $<1$ were avoided (Petrides 1975). The ratios were then converted to binary numbers where selected $=1$ and avoided $=0$.

Grass and browse selection. Chi-square tests between grass, bamboo, palms, Zingiberales (ginger hereafter), lianas and woody trees were used to determine if the differences between use and availability were significant. Statistical analyses were undertaken using SPSS 18.0.

New shoot volume. The vigour of each species was determined by taking averages of (1) the average number of new shoots, (2) the average values of change in new shoot length and (3) the average growth in basal area of the new shoot for each species between months. These were then used to calculate the average new shoot volume for each species using the formula for a cone $\left(V=\pi r^{2} h / 3\right)$.

$$
\begin{gathered}
=(\text { shoot basal area } \times \text { average monthly shoot length }) / 3 \\
\times \text { average number of new stems } \\
\frac{\pi r^{2} L}{3} \times S
\end{gathered}
$$

Where $\pi r^{2}$ is the basal area, $L$ is the average growth in shoot length per month and $S$ is the average number of new stems produced by the plant per month.

Plant vigour and size. The variables for plant vigour included the maximum monthly average for each species of growth in length of the new shoot (mm), number of new shoots produced and volume of new shoots $\left(\mathrm{mm}^{3}\right)$. Poaceae were not included in the vigour analyses and species with sample sizes of less than five were removed. A Principal Component Analysis (PCA) was used to reduce confounding effects of partial correlation between variables of plant regrowth vigour, thus reducing the number of covariates in the model to two. The principal component with which volume was most strongly associated had an eigenvalue $<1$ and was therefore removed from the regrowth vigour analyses. The length of new growth and number of stems produced was analysed using a Generalized Linear Model (GLM) with the preference ratio, a binary logistic, as the dependent variable using SPSS 18.0. The regrowth vigour of woody trees was first analysed to compare to previous studies, then vigour of gingers, palms and lianas was included in 


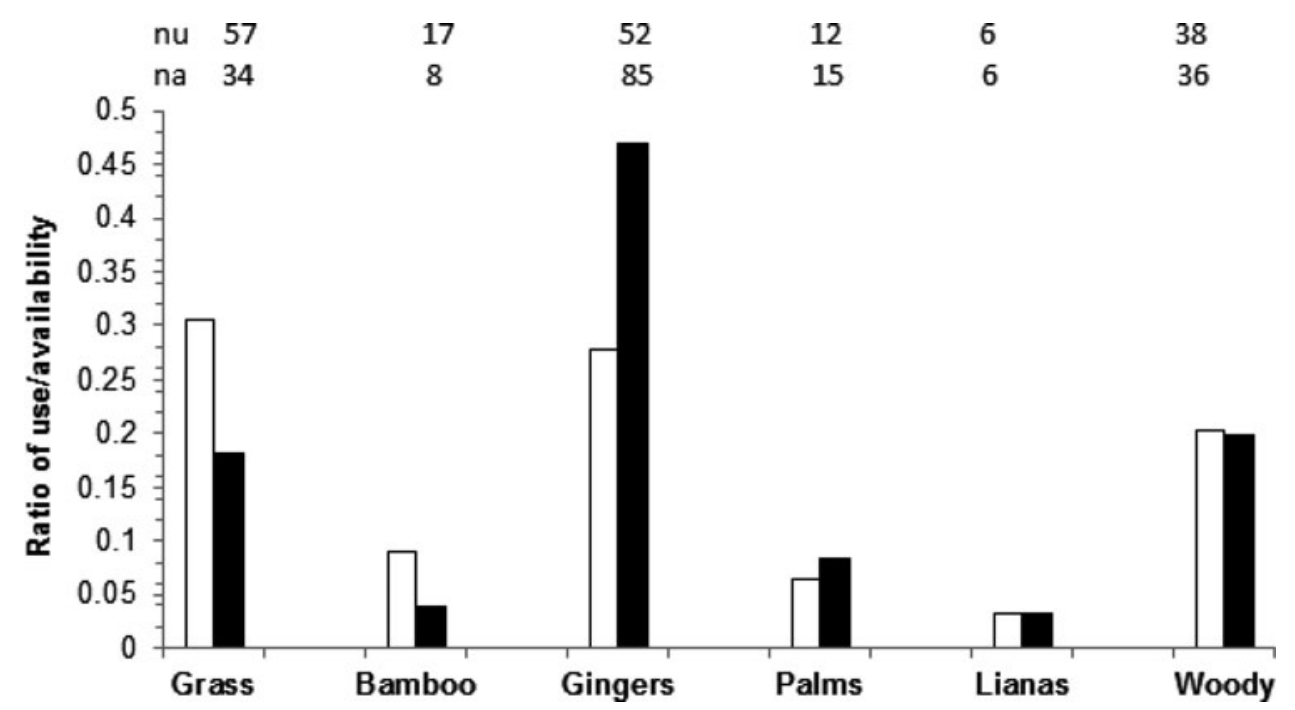

Figure 2. The ratio of plants selected by the Bornean elephant and plant availability in the Lower Kinabatangan Wildlife Sanctuary, Sabah. Plant species are in plant groups (grass, bamboo, gingers, palms, lianas and woody species). Used ratios of plants are white bars and available ratios of plants are black bars. The number of individual plants used (nu) and number of individual plants available (na) for each plant group are shown. Grasses are Phragmites karka; bamboo Dinochloa scabrida; gingers include Alpinia ligulata, Costus speciosus and Donax canniformis; palms include Licuala sp., Arenga sp. and Daemonorops sp.; lianas include Fordia splendidissima and woody includes Memecylon sp., Dillenia excelsa, Gardenia elata, Syzygium sp., Bridelia stipularis, Mallotus muticus, Lepisanthes fruticosa, Alangium javanicum and Garcinia parvifolia.

analyses due to these other plant types being available to elephants in a rain-forest environment. Plant size, using the basal diameter of the main stem $(\mathrm{mm})$, was also analysed using a GLM with the preference ratio as the dependent variable.

\section{RESULTS}

A total of 182 plants were eaten and 185 available plants were measured. Eighteen species were included in the analyses. Plant species were grouped into plant types for used and available comprising Poaceae (Phragmites karka, a grass and Dinochloa scabrida, a bamboo), gingers, palms, lianas and woody trees (Figure 2). The elephant favoured the grass and bamboo more than other plant types (Pearson chi-square, $\mathrm{N}=18, \chi^{2}{ }_{1}=0.920, \mathrm{P}=0.012$ ) although these were less common in the landscape (43 available Poaceae samples) compared with other species (142 available samples). The elephant preferred six and avoided 10 of the most common species along transects (Figure 3). Palms such as Licuala sp., and gingers such as Costus speciosus and Donax canniformis, were selected. Woody trees were less common, but Garcinia parvifolia, Lepisanthes sp. and Alangium sp. were most abundant and selected.

TheBornean elephant did not select woody trees that recovered more vigorously (GLM Logistic Regression, Wald Chi-square, $\mathrm{df}=5$ : new shoot length $\chi^{2}{ }_{1}=1.17, \mathrm{P}=$ 0.278; number of new stems $\chi^{2}{ }_{1}=0.479, \mathrm{P}=0.489$ ), nor did it prefer more vigorously regrowing species when more plant forms were included in analyses such as gingers, lianas and palms (GLM Logistic Regression, Wald Chi-square, $\mathrm{df}=12$ : new shoot length $\chi^{2}{ }_{1}=0.598$, $\mathrm{P}=0.439$; number of new stems $\chi^{2}{ }_{1}=0.231, \mathrm{P}=$ 0.631). Plant size did not influence selection (GLM Logistic Regression, Wald Chi-square, $\mathrm{df}=13$ : basal diameter $\left.\chi^{2}{ }_{1}=0.117, P=0.733\right)$. Favoured species were therefore not larger, their new growth not longer, nor did they produce more new stems than avoided plants.

\section{DISCUSSION}

We expected the Bornean elephant to select plants proportional to their availability and prefer palms, gingers, lianas and woody trees over grasses, including bamboos. We proposed that plant size, rather than plant regrowth vigour, might influence food choices. We did not find support for the influence of plant regrowth vigour or plant size on elephant food-plant choices but we did determine that the elephant in LKWS preferred to feed on Poaceae (Phragmites karka and Dinochloa scabrida) proportionately more than their availability, compared with other measured species.

\section{Plant selection}

Bornean elephant in the LKWS fed mainly on a subset of available species. Food-plant selection was not explained by the relative abundance of plant species. This finding 
(a)
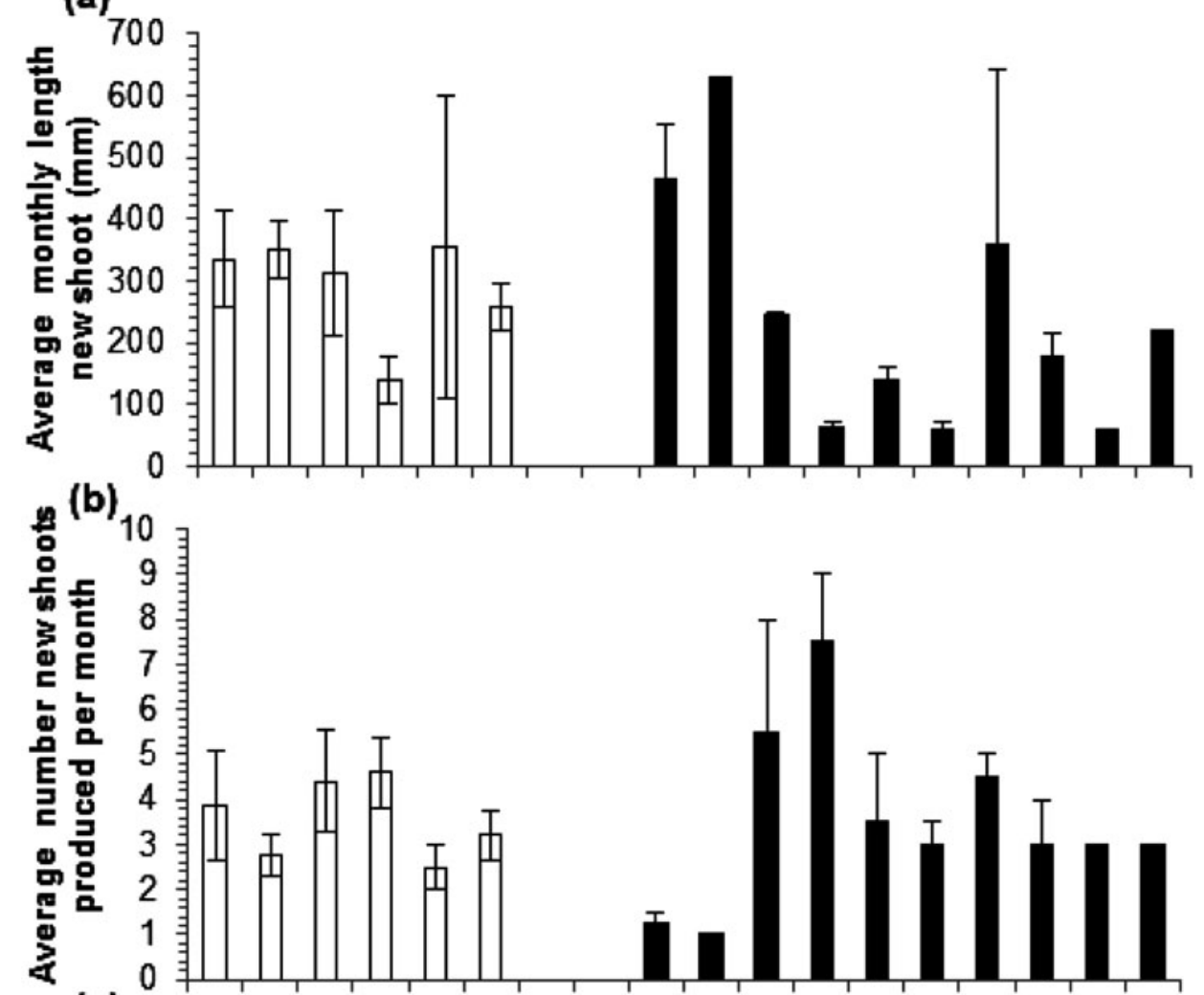

(c)

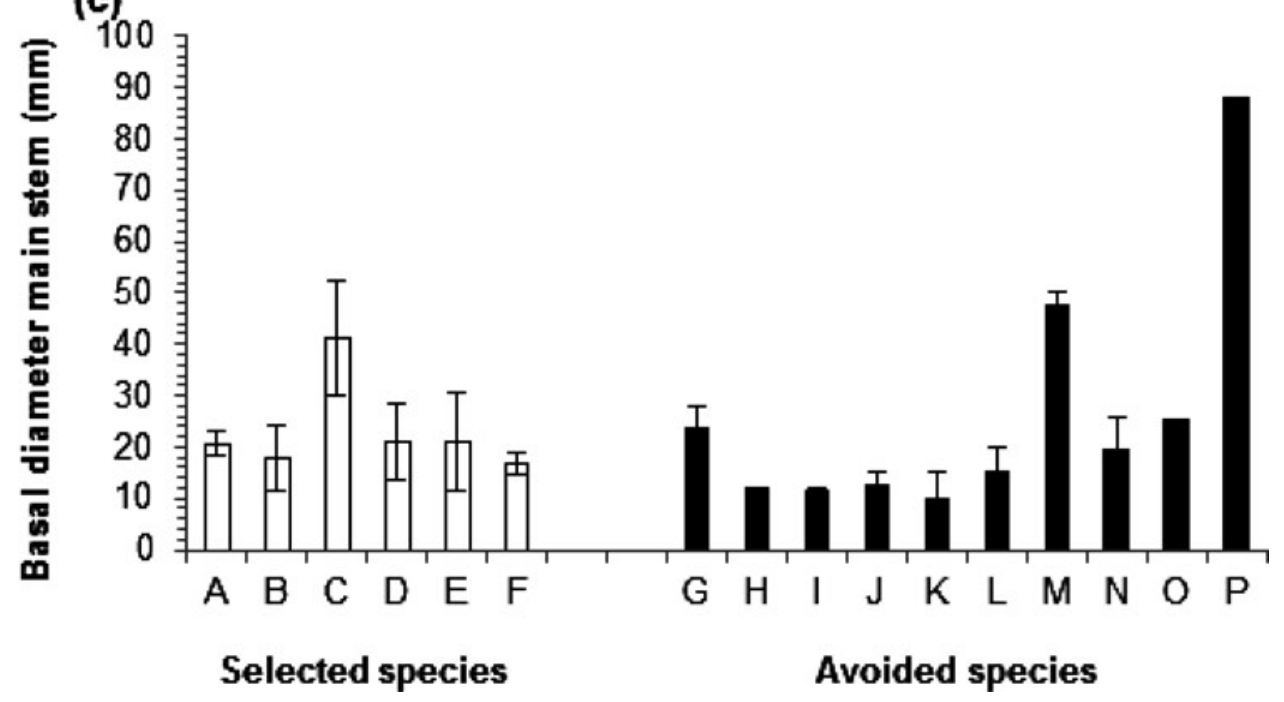

Figure 3. Plant selection and avoidance by the Bornean elephant in the Lower Kinabatangan Wildlife Sanctuary, Sabah, related to regrowth vigour for each species. Includes the average monthly growth in new shoot length ( $\mathrm{mm}$ ) (a) average monthly number of new shoots (b) and plant size using average basal diameter of the main stem $(\mathrm{mm})(\mathrm{c})$. Error bars represent standard error of individual samples within a species. Selected species in white and avoided species in black. Letters correspond to species names given in Table 1.

is consistent with several other studies on the feeding behaviour of African elephant (Loxodonta africana), African forest elephant (Loxodonta cyclotis) and Asian elephant (Elephas maximus) (Codron et al. 2011, McKay 1973, Olivier 1978, Seydack et al. 2000, Short 1981, Sukumar 1990, Williamson 1975, Wing \& Buss 1970).
Most studies of other elephant taxa (Loxodonta cyclotis) (Blake 2002) and (Elephas maximus) (Sukumar 1990) describe their preference for browse unless grass was the dominant vegetation, whereas Tchamba \& Seme (1993) and Olivier (1978) found grasses to be preferred despite being less abundant. In LKWS, although open grassed 
areas along forest margins (containing early-successional species such as bamboo) and riverine areas (containing semi-aquatic grasses such as reed), cover just $14 \%$ of the landscape (compared with $60 \%$ forested areas and $26 \%$ permanent swamp; English, unpubl. data). The Bornean elephant preferred feeding on the two Poaceae (Phragmites karka and Dinochloa scabrida) in riverine and open grassed areas along forest margins, than other species in the study. Thus, there is variation in the literature of the importance of grass species in the diet of the forest elephant relative to its abundance.

Asian elephants are believed to switch their diet preferences from grass to browse depending on seasonal changes in plant quality. For example, browse is consumed more in the dry season and grass in the early wet season (Sukumar 1990); although some other studies found elephants to prefer grasses despite seasonal influences (Olivier 1978, Tchamba \& Seme 1993). Our study period was in the dry season when more browsing might be expected but still the Bornean elephant preferred to feed on the two Poaceae mentioned. Seasonal influence is unlikely to be a major factor influencing plant quality in our study site because, despite a wet and dry season, rainfall is common throughout the year. Moreover, one of the species (Phragmites karka) is semi-aquatic and found in close proximity to a permanent water source. Moreover, these species are also perennial. It is unknown if there was any natural open areas within the forest historically in Sabah. Logging is believed to have started at least a century ago and the area could have been inhabited for many centuries by nomadic villages and the forest cleared for farming purposes. The river itself has been affected by a series of disturbances due to flooding and natural processes decimating forest and replacing with open areas containing early-successional species such as grasses, including bamboos (R. Nilus pers. comm.). However, regardless of their origin, these areas provide the elephants with much of their preferred food plants.

A second scenario that might explain why the Bornean elephant prefers Poaceae in the LKWS is that the quality of other plants within the elephant herd's range may be poor, because the remnant habitat is poor or the overall habitat quality may have declined. Higher quality habitat may have been replaced by oil palm cultivation (Elaeis guineensis) and forced the elephant to use areas that may be less optimal for foraging, or it may be exceeding the forests capacity to support it. An increase in the LKWS elephant population in the last $10 \mathrm{y}(<100->200$ individuals) (Ancrenaz pers. comm.) may have depleted preferred browse species and increased its use of Poaceae species to compensate. Bulk feeding on species such as reed and bamboo may enable elephants to meet intake requirements unable to be met by more abundant species.

Another scenario involves the influence of river hydrology on soil and plant quality through sediment and nutrient deposition by water filtered through the riparian zone. Rain-forest soil quality can range from highly leached, infertile soils to fertile, less-weathered, alluvial soils (Ashton 2004). Grasses, such as the common reed, in a floodplain landscape may be preferred due to the soil quality in riverine areas having higher nutrient concentration compared with soils further from the river where browse species are found. Moreover, vegetation growing on nutrient-deficient soils has been found to contain more chemical defences compared with plants found in areas with nutrient-rich soils (Coley et al. 1985, Owen-Smith \& Cooper 1987). Therefore, some plant species may be relatively high in chemical defences and of poorer quality to elephant within our study site. In addition, regular feeding increases soil nitrogen cycling and denitrification from herbivore dung deposition and urine, leading to elements being returned to the soil in readily available forms (McNaughton et al. 1988, Reuss 1986, Risser \& Parton 1982). The result is improved nitrogen availability for plants in these areas (Hamilton \& Frank 2001, Holland \& Detling 1990, McNaughton et al. 1997, Risser \& Parton 1982). Whatever the cause of plant preference by the Bornean elephant in LKWS it is clear that the common reed and bamboo are a significant part of its diet in a way not previously appreciated.

\section{Plant regrowth vigour and size}

The Bornean elephant of the LKWS did not prefer more vigorously regrowing plant species. This finding supports predictions based on body size and metabolism where the elephant represents an upper extreme in their tolerance of lower-quality food compared with smaller herbivores (Bell 1971, Demment \& van Soest 1985, Jarman 1974). For a larger animal that can accept a lower-quality diet, almost the whole plant is a homogenously acceptable food item, whereas for a smaller animal requiring a higher-quality diet a plant is a set of heterogeneous parts, from among which the more nutritious components must be selected (Bell 1971, Jarman 1974). High-quality parts of plants generally form smaller food items than do the low-quality parts. Thus, it is fitting that the diet selection of elephant, which must select their food for quantity rather than quality, should not be influenced by selection for more vigorous plants which are generally of higher nutritional quality (Price 1991).

Previous studies have found conflicting results in plant size preferences of elephants. The African forest elephant has been reported to prefer woody species with a smaller diameter at breast height (dbh) (Blake \& Inkamba-Nkulu 2004, Wing \& Buss 1970). In contrast, the Bornean elephant shows a preference for larger dbh in one woody species (Macaranga sp.) (Matsuyabashi et al. 2006). Compared with small herbivores, an elephant may 
consume more modules of a plant as it has a larger bite size and use of a prehensile trunk. Thus elephant might respond positively to a plant's size because more edible components are available (bark, leaves, new shoots, fruit and roots) (Vivas et al. 1991, Wilson \& Kerley 2003). We expected Bornean elephant to prefer larger plants as part of its optimal foraging strategy, as they should be less selective and conserve energy rather than seeking resources elsewhere (Charnov 1974). However, we found that selection and avoidance was not influenced by the size of the plant.

Our results suggest Bornean elephant foraging behaviour occurs at a larger spatial scale than at the plant level. Shrader et al. (2012) proposed that because elephant were generally forced to feed less selectively to provide sufficient intake, it is possible that it will make foraging decisions at the habitat or site level rather than at the individual plant level and this may also be the case with the elephants in our study.

\section{Implications for management of habitat and population}

Our findings about Bornean elephant food choices have direct implications for the restoration and management of elephant habitat in Sabah. Our results suggest that preservation of open areas along forest margins where Dinochloa scabrida is common (Dransfield 1992) and riverine areas where Phragmites karka are common is necessary for elephant conservation. Some forest disturbance is not deleterious as increased sunlight in ecotone and open areas encourages growth of earlysuccessional species such as those preferred by the Bornean elephant. Currently open areas along forest margins are actively planted with tree species as this is considered the foundation state for restoration. We recommend, however, that wildlife and habitat rehabilitation managers set aside some open areas for Poaceae throughout the LKWS. Replanting of trees along the river bank is important for minimizing erosion and providing opportunities for food or movement to a number of species, such as primates. It is also visually appealing for ecotourism in the area. The importance of bamboos and reeds, and perhaps other species within Poaceae, for elephants, however, should be incorporated into management and restoration planning.

\section{ACKNOWLEDGEMENTS}

We thank Victoria University of Wellington for supporting the study and providing a doctoral scholarship and a completion scholarship for Megan English, and are grateful to the Economic Planning Unit and Sabah
Wildlife Department for permitting the research in the Lower Kinabatangan Wildlife Sanctuary. Research was made possible by field assistants provided by HUTAN Kinabatangan Orang-utan Conservation Project and their Elephant Conservation Unit. We thank Danau Girang Field Centre for providing a vehicle and field staff, Dr John Sugau, Sabah Forestry Department, for identifying plant species and Professor Phillip GarnockJones from Victoria University of Wellington. This project was supported by funds to Megan English and Dr Wayne Linklater from Zoos Victoria, Australia.

\section{LITERATURE CITED}

ALFRED, R.\& AHMAD, A. H. 2010. Density and population estimation of the Bornean elephants (Elephas maximus borneensis) in Sabah. OnLine Journal of Biological Sciences 10:92-102.

ANCRENAZ, M., CALAQUE, R. \& LACKMAN-ANCRENAZ, I. 2004. Orangutan nesting behaviour in disturbed forest of Sabah, Malaysia: implications for nest consensus. International Journal of Primatology 25:983-1000.

ASHTON, S. 2004. Soils in the tropics. Pp. 56-68 in Losos, E. C. \& Leigh, E. G. (eds). Tropical forest diversity and dynamism, findings from a large-scale plot network. University of Chicago Press, Chicago.

BAKER, W. L. 1972. Eastern forest insects. U.S Department of Agriculture and Forest Service Miscellaneous Publication 1175:1-642.

BELL, R. H. V. 1971. A grazing ecosystem in the Serengeti. Scientific American 225:86-93.

BERGSTRÖM, R. \& HJELJORD, O. 1987. Moose and vegetation interactions in northwestern Europe and Poland. Swedish Wildlife Research Supplement 1:213-217.

BLAKE, S. 2002. The ecology of forest elephant distribution and its implications for conservation. Ph.D thesis, University of Edinburgh, Edinburgh.

BLAKE, S. \& INKAMBA-NKULU, C. 2004. Fruit, minerals and forest elephant trails: do all roads lead to Rome? Biotropica 36:392-401.

CHARNOV, E. L. 1974. Optimal foraging, the marginal value theorem. Theoretical Population Biology 9:129-136.

CHEN, J., DENG, X., ZHANG, L. \& BAI, Z. 2006. Diet composition and foraging ecology of Asian elephants in Shangyong, Xishuangbanna,China. Acta Ecologica Sinica 26:309-316.

CODRON, J., CODRON, D., LEE-THORP, J. A., SPONHEIMER, M., KIRKMAN, K., DUFFY, K. J. \& SEALY, J. 2011. Landscape-scale feeding patterns of African elephant inferred from carbon isotope analysis of faeces. Oecologia 165:89-99.

COLEY, P. D. \& AIDE, T. M. 1990. A comparison of herbivory and plant defenses in temperate and tropical broad-leaved forests. Pp. 25-49 in Price, P. W., Lewinsohn, T. M., Fernandes, G. W. \& Benson, W. W. (eds). Plant-animal interactions: evolutionary ecology in tropical and temperate regions. Wiley, New York.

COLEY, P.D., BRYANT, J. P.\& CHAPIN, F. S. 1985. Resource availability and plant anti-herbivore defense. Science 230:895-899. 
CRAIGHEAD, F. C. 1950. Insect enemies of eastern forests. U.S Department of Agriculture and Forest Service Miscellaneous Publication 657:1-679.

DANELL, K., HUSS-DANELL, K. \& BERGSTRÖM, R. 1985. Interactions between browsing moose and two species of birch in Sweden. Ecology 66:1867-1878.

DEMMENT, M. W. \& VAN SOEST, P. J. 1985. A nutritional explanation for body-size patterns of ruminant and nonruminant herbivores. American Naturalist 125:641-672.

DRANSFIELD, S. 1992. The bamboos of Sabah. Forestry Department, Sabah.

DU TOIT, J. T. \& OWEN-SMITH, N. 1989. Body size, population metabolism and habitat specialization among large African herbivores. American Naturalist 133:736-740.

ESTES, J. G., OTHMAN, N., ISMAIL, S., ANCRENAZ, M., GOOSSENS, B., AMBU, L. N. \& PALMIOTTO, P. A. 2012. Quantity and configuration of available elephant habitat and related conservation concerns in the Lower Kinabatangan floodplain of Sabah, Malaysia. PloS One 7: e44601.

FURNISS, R. L. \& CAROLIN, V. M. 1977. Western forest insects. U.S Department of Agriculture and Forest Service Miscellaneous Publication 1339:1-654.

HAMILTON, E. W. \& FRANK, D. A. 2001. Can plants stimulate soil microbes and their own nutrient supply? Evidence from a grazing tolerant grass. Ecology 82:2397-2402.

HOLLAND, E. A. \& DETLING, J. K. 1990. Plant response to herbivory and below ground nitrogen cycling. Ecology 74:855-862.

JARMAN, P. J. 1974. The social organisation of antelope in relation to their ecology. Behaviour 48:215-266.

JOHNSON, D. H. 1980. The comparison of usage and availability measurements for evaluating resource preference. Ecology 61:6571.

KEEN, F. P. 1952. Insect enemies of western forests. U.S Department of Agriculture and Forest Service Miscellaneous Publication 273:1280.

MAKHABU, S.W., SKARPE, C., HYTTEBORN, H. \& MPOFU, Z. D. 2006. The plant vigour hypothesis revisited - how is browsing by ungulates and elephants related to woody species growth rate? Plant Ecology 184:163-172.

MATSUYABASHI, H., LAGAN, P. \& SUKOR, J. R. 2006. Utilisation of Macaranga trees by the Asian elephant (Elephas maximus) in Borneo. Mammal Study 31:115-118.

MCKAY, G. M. 1973. The ecology and behavior of the Asiatic elephant in southeastern Ceylon. Smithsonian Contributions to Zoology 125:1113.

MCNAUGHTON, S. J. 1983. Compensatory plant growth as a response to herbivory. Oikos 40:329-336.

MCNAUGHTON, S. J., REUSS, R. W. \& SEAGLE, S. W. 1988. Large mammals and process dynamics in African ecosystems. Bioscience 38:794-800.

MCNAUGHTON, S. J., BANIKWA, F. F. \& MCNAUGHTON, M. M. 1997. Promotion of the cycling of diet enhancing nutrients by African grazers. Science 278:1798-1800.
OLIVIER, R. C. D. 1978. On the ecology of the Asian elephant. Ph.D thesis, University of Cambridge, Cambridge.

OWEN-SMITH, N. \& COOPER, S. M. 1987. Palatability of woody plants to browsing ungulates in South African savannah. Ecology 68:319331.

PETRIDES, G. A. 1975. Principal foods versus preferred foods and their relations to stocking rate and range condition. Biological Conservation 7:161-169.

PRADHAN, N. M., WEGGE, P., MOE, S. R. \& SHRESTHA, A. K. 2008. Feeding ecology of two endangered sympatric megaherbivores: Asian elephant (Elephas maximus) and greater one-horned rhinoceros (Rhinoceros unicornis) in lowland Nepal. Wildlife Biology 14:147-154.

PRICE, P. W. 1991. The plant vigour hypothesis \& herbivore attack. Oikos 62:244-251.

PRICE, P. W., ROININEN, H. \& TAHVANAINEN, J. 1987. Why does the bud-galling sawfly, Euura mucronata, attack long shoots? Oecologica 74:1-6.

REUSS, R. W. 1986. The role of large herbivores in nutrient cycling of tropical savannas. Pp. 67-91 in Walker, R. H. (ed.). Determinants of tropical savannas. IRL Press, Oxford.

RISSER, P. G. \& PARTON, W. J. 1982. Ecosystem analysis of the tall grass prairie-nitrogen-cycle. Ecology 63:1342-1351.

SEYDACK, A. H., VERMUELLEN, C. \& HUISMAN, J. 2000. Habitat quality and the decline of an African elephant population: implications for conservation. South African Journal of Wildife Research 30:34-42.

SHORT, J. 1981. Diet and feeding behaviour of the forest elephant. Mammalia 45:176-185.

SHRADER, A. M., BELL, C., BERTOLLI, L. \& WARD, D. 2012. Forest or the trees: at what scale do elephants make foraging decisions? Acta Oecologica 42:3-10.

SOORYANARAYAMA, S. 1995. Floods in Malaysia: patterns and implications. Malaysian Journal of Tropical Geography 26:35-46.

SUKUMAR, R. 1990. Ecology of the Asian elephant in southern India. II. Feeding habits and crop raiding patterns. Journal of Tropical Ecology 6:33-53.

SUKUMAR, R. \& RAMESH, R. 1992. Stable carbon isotope ratios in Asian elephant collagen: implications for dietary studies. Oecologia 91:536-539.

TCHAMBA, M. N. \& SEME, P. M. 1993. Diet and feeding behaviour of the forest elephant in the Santchou Reserve, Cameroon. African Journal of Ecology 31:165-171.

VIVAS, H. J., SAETHER, B. E. \& ANDERSON, R. 1991. Optimal twig size selection of a generalist herbivore, the moose Alces alces: implications for plant-herbivore interactions. Journal of Animal Ecology 60:395408.

WILLIAMSON, B. R. 1975. Seasonal distribution of elephant in Wankie National Park. Arnoldia 7:1-16.

WILSON, S. L. \& KERLEY, G. L. H. 2003. Bite diameter selection by thicket browsers: the effect of body size and plant morphology on forage intake and quality. Forest Ecology Management 181:51-65.

WING, L. D\&BUSS, I. O. 1970. Elephants and forest. Wildlife Monographs 19:1-92. 


\section{Literature Cited}

Aarts, G., MacKenzie, M., McConnell, B., Fedak, M., \& Matthiopoulos, J. (2008).

Estimating space-use and habitat preference from wildlife telemetry data. Ecography, 31: 140-160.

Ackerman, J. D., Mesler, M. R., Lu, K. L., \& Montalvo, A. M. (1982). Food-foraging behaviour of male Euglossini (Hymenoptera, Apidae): vagabonds or trapliners? Biotropica, 14: $241-248$.

Acres, B. D., \& Folland, C. J. (1975). The Soils of the Sabah: Sandakan and Kinabatangan Districts. Land Resources Division, Directorate of Overseas Surveys.

Adler, P., Raff, D., \& Lauenroth, W. (2001). The effect of grazing on the spatial heterogeneity of vegetation. Oecologia, 128: $465-479$.

Alfred, R., \& Ahmad, A. H. (2010). Density and population estimation of the Bornean elephants (Elephas maximus borneensis) in Sabah. Online Journal of Biological Sciences, 10: 92-102.

Alfred, R., Ahmad, A. H., Payne, J., Williams, C., Ambu, L. N., How, P. M., \& Goossens, B. (2012). Home range and ranging behaviour of Bornean elephant (Elephas maximus borneensis) females. PloS One, 7: e31400.

Ambu, L. N., \& Andau, P.M. (2002). Asian Elephant Action Plan. Sabah Wildlife Department.

Ancrenaz, M., Calaque, R., \& Lackman-Ancrenaz, I. (2004). Orangutan nesting behaviour in disturbed forest of Sabah, Malaysia: Implications for nest consensus. International Journal of Primatology, 25: 983 -1000.

Anderson, J. E. (1991). A conceptual framework for evaluating and quantifying naturalness. Conservation Biology, 5: 347-352. 
Archibald, S. (2008). African grazing lawns-How fire, rainfall, and grazer numbers interact to affect grass community states. Journal of Wildlife Management, 72: 492-501.

Ashton, S. (2004). Soils in the tropics. In Losos, E. C. \& Leigh, E. G. (eds). Tropical forest diversity and dynamism, findings from a large-scale plot network. University of Chicago Press, Chicago. pp. 56-68.

Aycrigg, J. L., \& Porter, W. F. (1997). Sociospatial dynamics of white-tailed deer in the Central Adirondack Mountains. New York. J. Mammal., 78: 468-482.

Azmi, R. (1998). Natural Vegetation of the Kinabatangan Floodplain. Part 1: Background and Preliminary Checklist. Report. Kota Kinabalu, Sabah: WWF-Malaysia.

Bailey, D.W., Rittenhouse, L. R., Hart, R. H., Swift, D. M., \& Richards, R. W. (1989). Association of relative food availabilities and locations by cattle. Journal of Range Management, 42: 480-482.

Bailey, D. W., Gross, J. E., Laca, E. A., Rittenhouse, L. R., Coughenour, M. B., Swift, D. M., \& Sims, P. L. (1996). Mechanisms that result in large herbivore grazing distribution patterns. Journal of Range Management, 49: 386-400.

Baker, W. L. (1972). Eastern forest insects. U.S Department of Agriculture and Forest Service Miscellaneous Publication, 1175: 1-642.

Bar-David, S., Bar-David, I., Cross, P. C., Ryan, S.J., Knechtel, C. U., \& Getz, W. M. (2009). Methods for assessing movement path recursion with application to African buffalo in South Africa. Ecology, 90: 2467-2479.

Barnes, R. F. W., Blom, A., Alers, M. P. T., \& Barnes, K. L. (1995). An estimate of the numbers of forest elephants in Gabon. Journal of Tropical Ecology, 11: 27-37. 
Barraquand, F., \& Benhamou, S. (2008). Animal movements in heterogeneous landscapes: identifying profitable places and homogeneous movement bouts. Ecology, 89: 3336-3348

Barratt, D. G., \& Hall-Martin, A. (1991). The effect of indigenous browsers on valley bushveld in the Addo Elephant National Park. In Proceedings of the First Valley Bushveld Symposium. Grassland Society of South Africa. Howick. pp. 14-1.

Bartumeus, F., Da Luz, M. G. E., Viswanathan, G. M., \& Catalan, J. (2005). Animal search strategies: A quantitative random-walk analysis. Ecology, 86: 3078-3087.

Bates, L. A., Poole, J. H., \& Byrne, R. W. (2008). Elephant cognition. Current Biology, 18: 544-546.

Bates, D., Maechler, M., Bolker, B., \& Walker, S. (2014). lme4: Linear mixed-effects models using Eigen and S4. R package version 1.0-6. http:/CRAN.R-project.org/package=lme4.

Bazely, D. R., \& Jefferies, R. L. (1989). Lesser snow geese and the nitrogen economy of a grazed salt marsh. The Journal of Ecology, 77: 24-34.

Beals, E. W. (1984). Bray-Curtis ordination: an effective strategy for analysis of multivariate ecological data. Advances in Ecological Research, 14: 55.

Bell, R. H. V. (1971). A grazing ecosystem in the Serengeti. Scientific American, 225: 86-93.

Bell, W. (1990). Searching behaviour patterns in insects. Annual Review Entomology, 35: $447-467$.

Belsky, A.J., Canham, C.D. (1994). Forest gaps and isolated savanna trees. Bioscience, 44: 77-84. 
Belovsky, G. E. (1984). Herbivore optimal foraging: A comparative test of three models. American Naturalist, 124: 97-115.

Benedict, F. G. (1936). The physiology of the elephant. The Physiology of the Elephant.pp 302.

Benhamou, S. (1990). An analysis of the movement of the wood mouse Apodemus sylvaticus in its home range. Behavioural Processes, 22: 235-250.

Benhamou, S., \& Riotte-Lambert, L. (2012). Beyond the utilisation distribution: Identifying homerange areas that are intensively exploited or repeatedly visited. Ecological Modelling, 227: $112-116$.

Ben-Shahar, R. (1993). Patterns of elephant damage to vegetation in northern Botswana. Biological Conservation, 65: 249-256.

Bergström, R., \& Danell, K. (1987). Effects of simulated winter browsing by moose on morphology and biomass of two birch species. The Journal of Ecology, 75: 533-544.

Bergström, R., \& Hjeljord, O. (1987). Moose and vegetation interactions in North-western Europe and Poland. Swedish Wildlife Research Supplement, 1: 213-217.

Bergström, R., Skarpe, C., \& Danell, K. (2000). Plant responses and herbivory following simulated browsing and stem cutting of Combretum apiculatum. Journal of Vegetation Science, 11: 409-414.

Beyer, H. L., Haydon, D. T., Morales, J. M., Frair, J. L., Hebblewhite, M., Mitchell, M., \& Matthiopoulos, J. (2010). The interpretation of habitat preference metrics under useavailability designs. Philosophical Transactions of the Royal Society B: Biological Sciences, 365: 2245-2254.

Blake, S. (2002). The ecology of forest elephant distribution and its implications for conservation. ICAPB, Edinburgh, University of Edinburgh. pp. 303. 
Blake, S., \& Inkamba-Nkulu, C. (2004). Fruit, minerals and forest elephant trails: do all roads lead to Rome? Biotropica, 36: 392-401.

Bingman, V. P., \& Cheng, K. (2005). Mechanisms of animal global navigation: comparative perspectives and enduring challenges. Ethology Ecology \& Evolution, 17: 295-318.

Biquand, S., \& Biquand-Guyot, V. (1992). The influence of peers, lineage and environment on food selection of the criollo goat (Capra hircus). Applied Animal Behaviour Science, 34: $231-245$.

Bond, W.J. (1993). Keystone species. In: Schulze, E.D., Mooney, H.A. (Eds.), Biodiversity and Ecosystem Function. Springer-Verlag, pp. 237-253

Börger, L., Dalziel, B. D., \& Fryxell, J. M. (2008). Are there general mechanisms of animal home range behaviour? A review and prospects for future research. Ecology letters, 11: 637650.

Bos, D., Drent, R. H., Rubinigg, M., \& Stahl, J. (2005). The relative importance of food biomass and quality for patch and habitat choice in Brent Geese Branta bernicla. Ardea, 93: 5-16.

Bovet, P., \& Benhamou, S. (1988). Spatial analysis of animals' movements using a correlated random walk model. Journal of theoretical biology, 131: 419-433.

Bradshaw, I. G. A. (2004). Not by bread alone: symbolic loss, trauma, and recovery in elephant communities. Society and animals, 12: 143-158.

Brooks, C. J., \& Harris, S. (2008). Directed movement and orientation across a large natural landscape by zebras, equus burchelli antiquorum. Animal Behaviour, 76: 277-285.

Bryan, J. E., Shearman, P. L., Asner, G. P., Knapp, D. E., Aoro, G., \& Lokes, B. (2013). Extreme differences in forest degradation in Borneo: Comparing practices in Sarawak, Sabah, and Brunei. PloS one, 8: e69679. 
Bryant, J. P. (1981). Phytochemical deterrence of snowshoe hare browsing by adventitious shoots of four Alaskan trees. Science, 213: 889-890.

Burke, D., \& Fulham, B. J. (2003). An evolved spatial memory bias in a nectar-feeding bird? Animal Behaviour, 66: 695-701.

Cagnacci, F., Boitani, L., Powell, R. A., \& Boyce, M. S. (2010). Animal ecology meets GPSbased radiotelemetry: a perfect storm of opportunities and challenges. Philosophical Transactions of the Royal Society B: Biological Sciences, 365: 2157-2162.

Cargill, S. M., \& Jefferies, R. L. (1984). The effects of grazing by lesser snow geese on the vegetation of a sub-arctic salt marsh. Journal of Applied Ecology, 21: 669-686.

Chamaillé-Jammes, S., Fritz, H., \& Madzikanda, H. (2009). Piosphere contribution to landscape heterogeneity: a case study of remote-sensed woody cover in a high elephant density landscape. Ecography, 32: 871-880.

Charnov, E. L. (1976). Optimal foraging, the marginal value theorem. Theroetical population biology, 9: 129-136.

Chen, J., Deng, X., Zhang, L., \& Bai, Z. (2006). Diet composition and foraging ecology of Asian elephants in Shangyong, Xishuangbanna,China. Acta Ecologica Sinica, 26: 309-316.

Clarke, K. R., \& Warwick, R. M. (1994). Similarity-based testing for community pattern: the two-way layout with no replication. Marine Biology, 118: 167-176.

Clarke, K. R, Gorley, R. N. (2006). PRIMER v6: User Manual/Tutorial. PRIMER-E, Plymouth.

Codron, J., Codron, D., Lee-Thorp, J. A., Sponheimer, M., Kirkman, K., Duffy, K. J., \& Sealy, J. (2010). Landscape-scale feeding patterns of African elephant inferred from carbon isotope analysis of faeces. Oecologia, 165: 89-99. 
Coley, P. D., \& Aide, T. M. (1991). Comparison of herbivory and plant defenses in temperate and tropical broad-leaved forests. Plant-animal interactions: evolutionary ecology in tropical and temperate regions. pp 25-49.

Coley, P. D., Bryant, J. P., \& Chapin, F. S. (1985). Resource availability and plant antiherbivore defense. Science, 230: 895-899.

Comba, L. (1999). Patch use by bumblebees (Hymenoptera Apidae): Temperature, wind, flower density and traplining. Ethology Ecology and Evolution, 11: 243-264.

Conybeare, A. M. (2004). Elephant impacts on vegetation and other biodiversity in the broadleaved woodlands of S-Central Africa. Biodiversity of the Four Corners Area: Technical Reviews Volume Two. Biodiversity Foundation for Africa, Bulawayo/Zambezi Society, Harare, Zimbabwe. pp. 477-508.

Coppock, D. L., Detling, J. K., Ellis, J. E., \& Dyer, M. I. (1983). Plant-herbivore interactions in a North American mixed-grass prairie. Oecologia, 56: 1-9.

Craighead, F. C. (1950). Insect enemies of eastern forests. U.S Department of Agriculture and Forest Service Miscellaneous Publication, 657: 1-679.

Cranbrook, E., Payne, J., \& Leh, C. M. U. (2008). Origin of the elephants Elephas maximus of Borneo. Saraw Mus J, 63: 1-25.

Cromsigt, J. P., \& Olff, H. (2008). Dynamics of grazing lawn formation: an experimental test of the role of scale-dependent processes. Oikos, 117: 1444-1452.

Cromsigt, J. P. G. M., \& Kuijper, D. P. J. (2011). Revisiting the browsing lawn concept: Evolutionary interactions or pruning herbivores? Perspectives in Plant Ecology, Evolution and Systematics, 13: 207-215. 
Dall, S. R., Giraldeau, L. A., Olsson, O., McNamara, J. M., \& Stephens, D. W. (2005). Information and its use by animals in evolutionary ecology. Trends in Ecology \& Evolution, 20: $187-193$.

Danell, K., Huss-Danell, K., \& Bergström, R. (1985). Interactions between browsing moose and two species of birch in Sweden. Ecology, 66: 1867-1878.

Davies, N. B., Houston, A. I. (1981). Owners and satellites-the economics of territory defense in the pied wagtail (Motacilla alba). Journal of Animal Ecology, 50: 157-180.

De Boer, W. F., Fairall, N., Van Aarde, R. J., \& Ntumi, C. P. (2005). Use of space and habitat by elephants (Loxodonta africana) in the Maputo Elephant Reserve, Mozambique. South African Journal of Wildlife Research, 35: 139-146.

De Knegt, H. J., Van Langevelde, F., Skidmore, A. K., Delsink, A., Slotow, R., Henley, S., \& Prins, H. H. (2011). The spatial scaling of habitat selection by African elephants. Journal of Animal Ecology, 80: 270-281.

de Mazancourt, C., Loreau, M., \& Abbadie, L. (1998). Grazing optimization and nutrient cycling: when do herbivores enhance plant production? Ecology, 79: 2242-2252.

Demment, M. W., \& van Soest, P. J. (1985). A nutritional explanation for body-size patterns of ruminant and nonruminant herbivores. American Naturalist, 125: 641-672.

Detling, J. K., \& Painter, E. L. (1983). Defoliation responses of western wheatgrass populations with diverse histories of prairie dog grazing. Oecologia, 57: 65-71.

DeVries, A. P. J, du Toit, J. T. \& Owen-Smith, N. (1989). Body size, population metabolism and habitat specialization among large African herbivores. The American Naturalist, 133: 736-740. 
Dressler, R. L. (1982). Biology of the orchid bees (Euglossini). Annual Review Ecological Systems 13: 373-394.

Dumont, B., \& Petit, M. (1998). Spatial memory of sheep at pasture. Applied Animal Behaviour Science, 60: 43-53.

Duncan, A. J., Hartley, S. E., \& Iason, G. R. (1998). The effect of previous browsing damage on the morphology and chemical composition of Sitka spruce (Picea sitchensis) saplings and on their subsequent susceptibility to browsing by red deer (Cervus elaphus). Forest Ecology and Management, 103: 57-67.

du Toit, J. T., \& Owen-Smith, N. (1989). Body size, population metabolism and habitat specialization among large African herbivores. The American Naturalist, 133: 736-740.

du Toit, J. T., Bryant, J. P., \& Frisby, K. (1990). Regrowth and palatability of Acacia shoots following pruning by African savanna browsers. Ecology, 71: 149-154.

El Aich, A., \& Rittenhouse, L. R. (1988). Use of habitats by free-grazing sheep. Applied Animal Behaviour Science, 21: 223-231.

English M, Kaplan G, Rogers LJ. (2014a). Is painting by elephants in zoos as enriching as we are led to believe? PeerJ 2:e471.

English, M., Ancrenaz, M., Gillespie, G., Goossens, B., Nathan, S., \& Linklater, W. L. (2014b). Foraging site recursion by forest elephants (Elephas maximus borneensis). Current Zoology, 60: 551-559.

English, M., Gillespie, G., Ancrenaz, M., Ismail, S., Goossens, B., Nathan, S \& Linklater, W. L. (2014c). Plant selection and avoidance by the Bornean elephant (Elephas maximus borneensis) in tropical forest: does plant recovery rate after herbivory influence food choices? Journal of Tropical Ecology, 30: 371-379.

English, M. (2015). Resource use and recursion by a mega-herbivore (Elephas maximus borneensis). Ph.D. thesis, Victoria University of Wellington, New Zealand. 
English, M., Gillespie, G., Goossens, B., Ismail, S., Ancrenaz, M., \& Linklater, W. (2015).

Recursion to food plants by free-ranging Bornean elephant. PeerJ, 3, e1030.

Erhart, E. M., Overdorff, D. J. (2008). Spatial memory during foraging in prosimian primates: Propithecus edwardsi and Eulemur fulvus rufus. Folia Primatologica, 79: 185-196.

Estes, J. G., Othman, N., Ismail, S., Ancrenaz, M., Goossens, B., Ambu, L. N., \& Palmiotto, P. A. (2012). Quantity and configuration of available elephant habitat and related conservation concerns in the Lower Kinabatangan floodplain of Sabah, Malaysia. PloS one, 7: e44601.

Everson, A.C. (1966). Effects of frequent clipping at different stubble heights on western wheatgrass (Agropyron smithii Rydb.). Agronomy Journal, 58: 33-35.

Fagan, W. F., Lewis, M. A., Auger-Méthé, M., Avgar, T., Benhamou, S., Breed, G., \& Mueller, T. (2013). Spatial memory and animal movement. Ecology letters, 16: 1316-1329.

Fauchald, P., \& Tveraa, T. (2003). Using first-passage time in the analysis of area-restricted search and habitat selection. Ecology, 84: 282-288.

Fernandes, G. W. \& Benson, W. W. (eds). Plant-animal interactions: evolutionary ecology in tropical and temperate regions. Wiley, New York.

Fernando, P., Vidya, T. C., Payne, J., Stuewe, M., Davison, G., Alfred, R. J., \& Melnick, D. J. (2003). DNA analysis indicates that Asian elephants are native to Borneo and are therefore a high priority for conservation. PLoS biology, 1: e6.

Firle, S., Bommarco, R., Ekbom, B., \& Natielo, M. (1998). The influence of movement and resting behaviour on the range of three carabid beetles. Ecology, 79: 2113-2122.

Fornoni, J. (2011). Ecological and evolutionary implications of plant tolerance to herbivory. Funct Ecol 25:399-407. 
Focardi, S., \& Tinelli, A. (1996). May random processes explain mating success in leks? Behavioural Processes, 36: 227-237.

Fretwell, S. D., \& Lucas, H. L. (1970). On territorial behaviour and other factors influencing habitat distribution in birds. Acta Biotheoretica, 19: 16-36.

Fryxell, J. M, Greever, J., \& Sinclair, A. R. E. (1988). Why are migratory ungulates so abundant? American Naturalist, 131: 781 - 798.

Fryxell, J. M. (1995). Aggregation and migration by grazing ungulates in relation to resources and predators. In: Serengeti 11: Dynamics, management and conservation of an ecosystem. Sinclair, R.E \& Arcese, P. (Eds). University of Chicago Press.

Fryxell, J. M., Wilmshurst, J. F., Sinclair, A. R., Haydon, D. T., Holt, R. D., \& Abrams, P. A. (2005). Landscape scale, heterogeneity, and the viability of Serengeti grazers. Ecology Letters, 8: 328-335.

Furniss, R. L., \& Carolin, V. M. (1977). Western forest insects. U.S Department of Agriculture and Forest Service Miscellaneous Publication, 1339: 1-654.

Galanti, V., Preatoni, D., Martinoli, A., Wauters, L. A., \& Tosi, G. (2006). Space and habitat use of the African elephant in the Tarangire-Manyara ecosystem, Tanzania: implications for conservation. Mammalian Biology-Zeitschrift für Säugetierkunde, 71: 99-114.

Garber, P. A. (1988). Foraging decisions during nectar feeding by tamarin monkeys (Saguinus mystax and Saguinus fuscicollis, Callitrichidae, Primates) in Amazonian Peru. Biotropica, 20: 100-106.

Garber, P. A., \& Jelinek, P. E. (2006). Travel patterns and spatial mapping in Nicaraguan mantled howler monkeys (Alouatta palliate). In: Estrada A, Garber PA, Pavelka MSM, Luecke Led. Springer US: New Perspectives in the Study of Mesoamerican Primates. pp. 287-309. 
Garrison, J. S. E., \& Gass, C. L. (1999). Response of a traplining hummingbird to changes in nectar availability. Behavioural Ecology, 10: 714-725.

Geist, V. (1971). Mountain sheep. A study in behavior and evolution. Univ. Chicago Press, Chicago. 383pp.

Gilbert, L. E. (1980). Ecological consequences of a coevolved mutualism between butterflies and plants. In: Gilbert LE, Raven PH ed. Coevolution of Animals and Plants. Austin: University of Texas Press. pp. 210-231.

Gill, F. B. (1988). Trapline foraging by hermit hummingbirds: Competition for an Undefended, Renewable Resource. Ecology, 69: 1933-1942.

Gillingham, M. P., \& Bunnell, F. L. (1989). Effects of learning on food selection and searching behaviour of deer. Canadian Journal of Zoology, 67: 24-32.

Gluesing, E. A., \& Balph, D. F. (1980). An aspect of feeding behaviour and its importance to grazing systems. Journal of Range Management, 33: 115-139.

Gordon, I. J., \& Lindsay, K. W. (1990). Could mammalian herbivores "manage" their own resources? Oikos, 59: 270-280.

Greco, B. J., Brown, T. K., Andrews, J. R., Swaisgood, R. R., \& Caine, N. G. (2013). Social learning in captive African elephants (Loxodonta africana). Animal cognition, 16: 459-469.

Greenwood, P. J., \& Harvey, P. M. (1982). The natal and breeding dispersal of birds. Annual Review of Ecological. Systems, 13: 1-21. 
Gregory, S. V., Swanson, F. J., McKee, W. A., \& Cummins, K. W. (1991). An ecosystem perspective of riparian zones. Bioscience, 41: 540-551.

Grünbaum, D. (2000). Advection-diffusion equations for internal state-mediated random walks. SIAM Journal of Applied Mathematics, 61: 43-73.

Hai, T. C., Ng, A., Prudente, C., Pang, C., \& Yee, J. T. C. (2001). Balancing the need for sustainable oil palm development and conservation: The Lower Kinabatangan floodplains experience. In ISP National Seminar.

Hamilton, E.W., \& Frank, D. A. (2001). Can plants stimulate soil microbes and their own nutrient supply? Evidence from a grazing tolerant grass. Ecology, 82: 2397-2402.

Hansson, L., Fahrig, L., Merriam, G. (Eds). (1995). Mosaic landscapes and ecological processes. Chapman and Hall, London.

Harris, G. M., Russell, G. J., van Aarde, R. I., \& Pimm, S. L. (2008). Rules of habitat use by elephants Loxodonta africana in southern Africa: insights for regional management. Oryx, 42: $66-75$.

Hart, B. L., Hart, L. A., \& Pinter-Wollman, N. (2008). Large brains and cognition: Where do elephants fit in? Neuroscience and Biobehavioural Reviews, 32: 86-98.

Hastings, A., Byers, J. E., Crooks, J. A., Cuddington, K., Jones, C. G., Lambrinos, J. G., Talley, T. S., \& Wilson, W. G. (2007). Ecosystem engineering in space and time. Ecology Letters, 10:153-164.

Heinrich, B. (1976). The foraging specialisations of individual bumblebees. Ecological Monographs, 46: 105-128.

Hik, D. S., \& Jefferies, R. L. (1990). Increases in the net above-ground primary production of a salt-marsh forage grass: a test of the predictions of the herbivore-optimization model. The Journal of Ecology, 180-195. 
Hing, S., Othman, N., Nathan, S. K., Fox, M., Fisher, M., \& Goossens, B. (2013). First parasitological survey of Endangered Bornean elephants Elephas maximus borneensis. Endangered Species Research, 21: 223-230

Hodder, R. M., \& Low, W. A. (1978). Grazing distribution of free-ranging cattle at three sites in the Alice Springs District, Central Australia. The Rangeland Journal, 1: 95-105.

Holdo, R. M., Holt, R. D., Coughenour, M. B., \& Ritchie, M. E. (2007). Plant productivity and soil nitrogen as a function of grazing, migration and fire in an African Savanna. Journal of Ecology, 95: 115-128.

Holland, E. A., \& Detling, J. K. (1990). Plant response to herbivory and below ground nitrogen cycling. Ecology, 74: 855-862.

Holling, C. S. (1996). Cross-scale morphology, geometry, and dynamics of ecosystems. In Ecosystem Management. Springer New York. pp. 351-423

Hulbert, I. A., \& Andersen, R. (2001). Food competition between a large ruminant and a small hindgut fermenter: the case of the roe deer and mountain hare. Oecologia, 128: 499508.

Huntly, N. (1991). Herbivores and the dynamics of communities and ecosystems. Annual Review Ecological Systems, 22: 447-503.

Huston, M. A., \& Huston, M. A. (1994). Biological diversity: the coexistence of species. Cambridge University Press.

Hutton, J., Adams, W. M., \& Murombedzi, J. (2005). Back to the Barriers? Changing Narratives in Biodiversity Conservation. Forum for development Studies, 32: 341-365. 
Illius, A. W. \& Gordon, I. J. (1990). Constraints on diet selection and foraging behaviour in mammalian herbivores. Behavioural Mechanisms of Food Selection (ed. R.N. Hughes). Springer-Verlag, Berlin. pp. 369-393.

IUCN Redlist. (2014). http://www.iucnredlist.org/

Jachman, H., \& Bell, R. H. V. (1985). Utilisation by elephants of the Brachystegia woodlands of the Kasungu National Park, Malawi. African Journal of Ecology, 23: 245-258.

Jameson, D. A. (1963). Responses of individual plants to harvesting. Botanical Reviews, 29: $532-594$.

Janis, C. M. (1976). The evolutionary strategy of the Equidae and the origins of rumen and cecal digestion. Evolution, 30: 757-774.

Janmaat, K. R., Byrne, R. W., \& Zuberbühler, K. (2006). Evidence for a spatial memory of fruiting states of rainforest trees in wild mangabeys. Animal Behaviour, 72: 797-807.

Janmaat, K. R., Ban, S. D., \& Boesch, C. (2013). Taï chimpanzees use botanical skills to discover fruit: What we can learn from their mistakes. Animal Cognition, 16: 851-860.

Janson, C.H. (1998). Experimental evidence for spatial memory in foraging wild capuchin monkeys Cebus apella. Animal Behaviour, 55: 1229-1243.

Janzen, D. H. (1971). Euglossine bees as long-distance pollinators of tropical plants. Science, 171: 203-205.

Jarman, P. J. (1974). The social organisation of antelope in relation to their ecology.

Behaviour, 48: 215-266.

Johnson, D. H. (1980). The comparison of usage and availability for evaluating resource preference. Ecology, 61: 65-71. 
Johnson, C. J., Parker, K. L., Heard, D. C., \& Gillingham, M. P. (2002). Movement parameters of ungulates and scale-specific responses to the environment. Journal of Animal Ecology, 71: 225-235.

Jones, C. G., Lawton, J. H., \& Shachak, M. (1996). Organisms as ecosystem engineers. In Ecosystem Management. Springer New York. pp. 130-147.

Kadmon, R. (1992). Dynamics of forager arrivals and nectar renewal in flowers of Anchusa stigosa. Oecologia, 92: 552-555.

Kamstra, L. D. (1973). Seasonal changes in quality of some important range grasses. Journal of Range Management, 26: 289-291.

Karban, R., \& Myers, J. H. (1989). Induced plant responses to herbivory. Annual Review of Ecology and Systematics, 20: 331-348.

Kareiva, P. M., \& Shigesada, N. (1983). Analysing insect movement as a correlated random walk. Oecologia, 56: 234-238.

Keen, F. P. (1958). Cone and seed insects of western forest trees. US Department of Agriculture, No. 1169.

Kertson, B. N., \& Marzluff, J. M. (2011). Improving studies of resource selection by understanding resource use. Environmental Conservation, 38: 18-27.

Koehler, H. H. (2000). Natural regeneration and succession-results from a 13 years study with reference to mesofauna and vegetation, and implications for management. Landscape and Urban Planning, 51: 123-130.

Kozakiewicz, M. (1995). Resource tracking in space and time. In Mosaic landscapes and ecological processes. Springer Netherlands. pp. 136-148. 
Kumar, M. A., Mudappa, D., \& Raman, T. S. (2010). Asian elephant Elephas maximus habitat use and ranging in fragmented rainforest and plantations in the Anamalai Hills, India. Tropical Conservation Science, 3: 143-58.

Kunin, W. E. (1998). Extrapolating species abundance across spatial scales. Science, 281: $1513-1515$.

Langley, R. B. (1999). Dilution of precision. GPS World, 10: 52-59.

Langrock, R., King, R., Matthiopoulos, J., Thomas, L., Fortin, D., \& Morales, J. M. (2012). Flexible and practical modeling of animal telemetry data: hidden Markov models and extensions. Ecology, 93: 2336-2342.

Latip, A, N., Bauer, S., \& Umzarulazijo Umar, M. (2013). Forest Management in Lower Kinabatangan Sabah, East Malaysia: Cost-Benefit Analysis. Journal of Social \& Development Sciences, 4: 376-386.

Launchbaugh, K. L., \& Howery, L. D. (2005). Understanding landscape use patterns of livestock as a consequence of foraging behaviour. Rangeland Ecology \& Management, 58: 99-108.

Laws, R. M. (1970). Elephants as agents of habitat and landscape change in East Africa. Oikos, 21: 1-15.

Leimgruber, P., Gagnon, J. B., Wemmer, C., Kelly, D. S., Songer, M. A., \& Selig, E. R. (2003). Fragmentation of Asia's remaining wildlands: implications for Asian elephant conservation. Animal Conservation, 6: 347-359.

Lemmon, P. E. (1956). A spherical densiometer for estimating forest overstory density. Forest Science, 2: 314-320.

Lemke, T. O. (1984). Foraging ecology of the long-nosed bat Glossophaga soricina, with respect to resource availability. Ecology, 65: 538-548. 
Li, Z., Han, J., Ding, B., \& Kays, R. (2012). Mining periodic behaviours of object movements for animal and biological sustainability studies. Data Min. Knowl. Discovery, 24: $355-386$.

Lima, S. L., \& Zollner, P. A. (1996). Towards a behavioural ecology of ecological landscapes. Trends in Ecology \& Evolution, 11: 131-135.

Makhabu, S. W., Skarpe, C., \& Hytteborn, H. (2006a). Elephant impact on shoot distribution on trees and on rebrowsing by smaller browsers. Acta oecologica, 30: 136-146.

Makhabu, S.W., Skarpe, C., Hytteborn, H., \& Mpofu, Z. D. (2006b). The plant vigour hypothesis revisited- how is browsing by ungulates and elephants related to woody species growth rate? Plant Ecology, 184: 163-172.

Malim, T. P. (2002). Kinabatangan Wildlife Management Plan: Mechanisms of the Plan and It's Benefits. Forum on Sustainable Management of Ecotourism in Wetlands: The Case of Lower Kinbatangan Floodplain. Kota Kinabalu: Institute for Development Studies (Sabah), World Wide Fund for Nature, Malaysia (WWFM) and Sabah Wildlife Department.

Manly, B. F. J., McDonald, L. L., Thomas, D. L., McDonald, T. L., \& Erickson, W. P., (2002). Resource selection by animals: statistical analysis and design for field studies. Second edition. Kluwer Press, Boston, Massachusetts, USA.

Manning, A. (1956). Some aspects of the foraging behaviour of bumble-bees. Behaviour, 9: $164-201$.

Mapaure, I. N., \& Campbell, B. M. (2002). Changes in miombo woodland cover in and around Sengwa Wildlife Research area, Zimbabwe in relation to elephants and fire. African Journal of Ecology, 40: 167-185.

Matsubayashi, H., Lagan, P., \& Sukpr, J. R. (2006). Utilisation of Macaranga trees by the Asian elephant (Elephas maximus) in Borneo. Mammal Study, 31: 115-118. 
McCulloch, C. E., Cain, M. L. (1989). Analyzing discrete movement data as a correlated random walk. Ecology, 70: 383-388.

McKay, G. M. (1973). Behaviour and ecology of the Asiatic elephant in southeastern Ceylon. Smithsonian Contributions to Zoology, 125: 1-113.

McNamara, J. M., \& Houston, A. I. (1996). State-dependent life histories. Nature, 380: 215221.

McNaughton, S. J. (1976). Serengeti migratory wildebeest: facilitation of energy flow by grazing. Science, 191:92-94.

McNaughton, S. J. (1979). Grazing as an optimization process: grass-ungulate relationships in the Serengeti. American Naturalist, 113: 691-703.

McNaughton, S. J. (1983). Compensatory plant growth as a response to herbivory. Oikos, 40 : $329-336$.

McNaughton, S. J. (1984). Grazing lawns: animals in herds, plant form, and coevolution. American Naturalist, 124: 863-886.

McNaughton, S. J. (1985). Ecology of a grazing ecosystem: The Serengeti. Ecological Monographs, 55: 259-294.

McNaughton, S. J., Ruess, R. W., \& Seagle, S. W. (1988). Large mammals and process dynamics in African ecosystems. BioScience, 38: 794-800.

McNaughton, S. J., Banyikwa, F. F., \& McNaughton, M. M. (1997). Promotion of the cycling of diet-enhancing nutrients by African grazers. Science, 278: 1798-1800. 
Menzel, R., Greggers, U., Smith, A., Berger, S., Brandt, R., Brunke, S., \& Watzl, S. (2005). Honey bees navigate according to a map-like spatial memory. Proceedings of the National Academy of Sciences of the United States of America, 102: 3040-3045.

Morales, J. M., \& Ellner, S. P. (2002). Scaling up animal movements in heterogeneous landscapes: the importance of behaviour. Ecology, 83: 2240-2247.

Morales, J. M., Haydon, D. T., Frair, J., Holsinger, K. E., \& Fryxell, J. M. (2004). Extracting more out of relocation data: building movement models as mixtures of random walks. Ecology, 85: 2436-2445.

Moss, C. J. (2012). Elephant memories: thirteen years in the life of an elephant family. University of Chicago Press.

Mouissie, A. M., Apol, M. E. F., Heil, G. W., \& van Diggelen, R. (2008). Creation and preservation of vegetation patterns by grazing. Ecological Modelling, 218: 60-72.

Nathan, R., Getz, W. M., Revilla, E., Holyoak, M., Kadmon, R., Saltz, D., \& Smouse, P. E. (2008). A movement ecology paradigm for unifying organismal movement research. Proceedings of the National Academy of Sciences, 105: 19052-19059.

Nicotri, M. E. (1980). Factors involved in herbivore food preference. Journal of Experimental Marine Biology and Ecology, 42: 13-26.

Oaten, A. (1977). Optimal foraging in patches: a case for stochasticity. Theoretical Population Biology, 12: 263-285.

O’Connor, T. G., Goodman, P. S., \& Clegg, B. (2007). A functional hypothesis of the threat of local extirpation of woody plant species by elephant in Africa. Biological Conservation, 136: $329-345$.

Ohashi, K., \& Thomson, J. D. (2005). Efficient harvesting of renewing resources. Behavioural Ecology, 16: 592-605. 
Okubo, A. (1980). Diffusion and ecological problems: mathematical models. SpringerVerlag, Berlin.

Okubo, A., \& Levin, S. A. (Eds.). (2001). Diffusion and ecological problems: modern perspectives (Vol. 14). Springer.

Olff, H., \& Ritchie, M. E. (1998). Effects of herbivores on grassland plant diversity. Trends in ecology \& evolution, 13: 261-265.

Olivier, R. C. D. (1978). On the ecology of the Asian elephant. Ph.D thesis, University of Cambridge.

Olofsson, J., Kitti, H., Rautiainen, P., Stark, S., \& Oksanen, L. (2001). Effects of summer grazing by reindeer on composition of vegetation, productivity and nitrogen cycling. Ecography, 24: 13-24.

Olofsson, J., E Hulme, P., Oksanen, L., \& Suominen, O. (2004). Importance of large and small mammalian herbivores for the plant community structure in the forest tundra ecotone. Oikos, 106: 324-334.

Osborn, F. V. (2004). Seasonal variation of feeding patterns and food selection by cropraiding elephants in Zimbabwe. African Journal of Ecology, 42: 322-327.

Othman, N., Mohamed, M., Ahmad, A. H., Nathan, S., Pierson, H. T., \& Goossens, B. (2008). A preliminary study on the morphometrics of the Bornean Elephant. Journal of Tropical Biology Conservation, 4: 1109-1113.

Othman, N., Fernando, P., Yoganand, K., Ancrenaz, M., Alfred, R. J., Nathan, S., \& Goossens, B. (2013). Elephant Conservation and Mitigation of Human-Elephant Conflict in Government of Malaysia-UNDP Multiple-Use Forest Landscapes Project Area in Sabah. GAJAH, 39: 19-23.

Owen-Smith, N., \& Novellie, P. (1982). What should a clever ungulate eat? American Naturalist.pp 151-178. 
Owen-Smith, N., \& Cooper, S. M. (1987). Palatability of woody plants to browsing ungulates in South African savannah. Ecology, 68: 319-331.

Owen-Smith, R. N. (1988). Mega-herbivores: The influence of very large body size on ecology. Cambridge; New York: Cambridge University Press.

Owen-Smith, N. (2002). Adaptive Herbivore Ecology: From Resources to Populations in Variable Environments. Cambridge: Cambridge University Press.

Owen-Smith, N., \& Chafota, J. (2012). Selective feeding by a mega-herbivore, the African elephant. Journal of Mammalogy, 93: 698-705.

Papastamatiou, Y. P., Meyer, C. G., Carvalho, F., Dale, J. J., Hutchinson, M. R., \& Holland, K. N. (2013). Telemetry and random-walk models reveal complex patterns of partial migration in a large marine predator. Ecology, 94: 2595-2606.

Parra, R. (1978). Comparison of foregut and hindgut fermentation in herbivores. The ecology of arboreal folivores, pp. 205-229.

Patterson, T. A., Thomas, L., Wilcox, C., Ovaskainen, O., \& Matthiopoulos, J. (2008). Statespace models of individual animal movement. Trends in ecology \& evolution, 23: 87-94.

Payne, J. (1989). A tourism feasability study for the proposed KinabatanganWildlife Sanctuary. In WWF - Malaysia Int. Rep., Kuala Lumpur, Malaysia.

Payne, J., \& Andau, P. (1997). Kinabatangan River Conservation Area. In Elasmobranch Biodiversity, Conservation and Management. Proceedings of the International Seminar and Workshop. Sabah, East Malaysia, pp. 243-244.

Payne, J., \& Davies, G. (2013). Conservation of rainforest mammals in Sabah: Long term perspectives. The Raffles Bulletin of Zoology, 29: 187-201.

Plumptre, A. J. (1993). The effects of trampling damage by herbivores on the vegetation of the Parc National des Volcans, Rwanda. African Journal of Ecology, 32: 115-129. 
Porter, L. M., \& Garber, P.A. (2013). Foraging and spatial memory in wild Weddell's saddleback tamarins Saguinus fuscicollis weddelli when moving between distant and out-ofsight goals. International Journal of Primatology, 34: 30-48.

Possingham, H. P. (1989). The distribution and abundance of resources encountered by a forager. American Naturalist, 133: 42-60.

Powell, J. A. (1997). The ecology of forest elephants Loxodonta africana cyclotis in Banyang- Mbo and Korup forests, Cameroon with particular reference to their role as seed dispersal agents. Doctoral dissertation, University of Cambridge.

Pradhan, N. M., Wegge, P., Moe, S. R., \& Shrestha, A. K. (2008). Feeding ecology of two endangered sympatric mega-herbivores: Asian elephant (Elephas maximus) and greater onehorned rhinoceros (Rhinoceros unicornis) in lowland Nepal. Wildlife Biology, 14: 147-154.

Price, P.W., Roininen, H., \& Tahvanainen, J. (1987). Why does the bud-galling sawfly, Euura mucronata, attack long shoots? Oecologica, 74: 1-6.

Price, P.W. (1991). The plant vigour hypothesis \& herbivore attack. Oikos, 62: 244-251.

Pringle, R. M. (2008). Elephants as agents of habitat creation for small vertebrates at the patch scale. Ecology, 89: 26-33.

Prins, H. H. T., Ydenberg, R. C., \& Drent, R. H. (1980). The interaction of brent geese Branta bernicla and sea plantain Plantago maritima during spring staging. Acta Bot Neerl, 29: 585-596.

Prins, H. H. T., Olff, H., Newbery, D. M., \& Brown, N. D. (1998). Species-richness of African grazer assemblages: towards a functional explanation. In Dynamics of tropical communities: the 37th symposium of the British Ecological Society, Cambridge University, 1996. (pp. 449-490). Blackwell Science Ltd.

Provenza, F. D., Scott, C. B., Phy, T. S., \& Lynch, J. J. (1996). Preference of sheep for foods varying in flavours and nutrients. Journal of Animal Science, 74: 2355-2361. 
Provenza, F. D., Villalba, J. J., Dziba, L.E., Atwood, S.B., \& Banner, R.E. (2003). Linking herbivore experience, varied diets, and plant biochemical diversity. Small Ruminant Research, 49: 257-274.

Pulliam, H. R., \& Danielson, B. J. (1991). Sources, sinks, and habitat selection: a landscape perspective on population dynamics. American naturalist, 137: 50-S66.

Pyke, G. H. (1984). Optimal foraging theory: A critical review. Annual Review of Ecological Systems 15: 523-575.

Racey, P. A., Swift, S. M. (1985). Feeding ecology of Pipistrellus pipistrellus (Chiroptera, Vespertilionidae) during pregnancy and lactation. 1. Foraging behaviour. Journal Animal Ecology, 54: 205-215.

Real, R., \& Vargas, J. M. (1996). The probabilistic basis of Jaccard's index of similarity. Systematic biology, 380-385.

Redfern, J. V., Grant, R., Biggs, H., \& Getz, W. M. (2003). Surface-water constraints on herbivore foraging in the Kruger National Park, South Africa. Ecology, 84: 2092-2107.

Riotte-Lambert, L. Benhamou, S., \& Chamaille-Jammes, S. (2013). Periodicity analysis of movement recursions. Journal of Theoretical Biology, 317: 238-243.

Risser, P. G., \& Parton, W. J. (1982). Ecosystem analysis of the tall grass prairie-nitrogencycle. Ecology, 63: 1342-1351.

Rood, E., Ganie, A. A., \& Nijman, V. (2010). Using presence-only modelling to predict Asian elephant habitat use in a tropical forest landscape: implications for conservation. Diversity and Distributions, 16: 975-984.

Ruess, R. W., \& McNaughton, S. J. (1984). Urea as a promotive coupler of plant-herbivore interactions. Oecologia, 63: 331-337. 
Ruess, R.W. (1986). The role of large herbivores in nutrient cycling of tropical savannas. In Walker, R. H (ed.). Determinants of tropical savannas. IRL Press, Oxford. pp. 67-91.

Runting, R. K., Meijaard, E., Abram, N. K., Wells, J. A., Gaveau, D. G., Ancrenaz, M., Posssingham, H. P., Wich, S. A., Ardiansyah, F., Gumal, M. T., Ambu, L. N., \& Wilson, K. A. In review. Alternative futures for tropical forests. Nature Scientific Reports.

Rutina, L. P., Moe, S. R., \& Swenson, J. E. (2005). Elephant Loxodonta africana driven woodland conversion to shrubland improves dry-season browse availability for impalas Aepyceros melampus. Wildlife Biology, 11: 207-213.

Sabah Department of Statistics (2001). http://www.statistics.gov.my

Sanderson, E. W., Redford, K. H., Vedder, A., Coppolillo, P. B., \& Ward, S. E. (2002). A conceptual model for conservation planning based on landscape species requirements. Landscape and urban planning, 58: 41-56.

Schoen, J. W., and Kirchhoff. M. D. (1985).Seasonal distribution and home-range patterns of Sitka black-tailed deer on Admirality Island, southeast Alaska. Journal of Wildlife Management, 49: 96-103.

Senft, R. L., Coughenour, M. B., Bailey, D. W., Rittenhouse, L. R., Sala, O. E., \& Swift, D. M. (1987). Large herbivore foraging and ecological hierarchies. BioScience, 37: 789-799.

Seydack, A. H., Vermuellen, C., \& Huisaman, J. (2000). Habitat quality and the decline of an African elephant population: implications for conservation. South African Journal of Wildlife Research, 30: 34-42.

Sha, J. C., Bernard, H., \& Nathan, S. (2008). Status and conservation of proboscis monkeys (Nasalis larvatus) in Sabah, East Malaysia. Primate Conservation, 23: 107-120. 
Shannon, G., Page, B., Slotow, R., \& Duffy, K. (2006). African elephant home range and habitat selection in Pongola Game Reserve, South Africa. African Zoology, 41: 37-44.

Sharma, R., Goossens, B., Kun-Rodrigues, C., Teixeira, T., Othman, N., Boone, J. Q., \& Chikhi, L. (2012). Two different high throughput sequencing approaches identify thousands of de novo genomic markers for the genetically depleted Bornean elephant. PloS one, 7: e49533.

Short, J. (1981). Diet and feeding behaviour of the forest elephant. Mammalia, 45: 176-185.

Shoshani, J., \& Eisenberg, J. F. (1982). Elephas maximus. Mammalian Species, 182: 1-8.

Shoshani, J., \& Tassy, P. (2005). Advances in proboscidean taxonomy \& classification, anatomy \& physiology, and ecology \& behaviour. Quaternary International, 126: 5-20.

Shrader, A. M., Bell, C., Bertolli, L. \& Ward, D. (2012). Forest or the trees: at what scale do elephants make foraging decisions? Acta Oecologica, 42: 3-10.

Sinclair, A. R. E. (1977). The African buffalo: a study of resource limitation of populations. University of Chicago Press, Chicago.

Sitompul, A. F. (2011). Ecology and conservation of Sumatran elephants (Elephas maximus sumatranus) in Sumatra, Indonesia. PhD Dissertation, University of Massachusetts Amherst.

Sitompul, A. F., Griffin, C. R., \& Fuller, T. K. (2013). Sumatran elephant ranging behavior in a fragmented rainforest landscape. Int. J. Biodiv. Conserv,5: 66-72.

Skalski, G. T., \& Gilliam, J. F. (2000). Modeling diffusive spread in a heterogeneous population: a movement study with stream fish. Ecology, 81: 1685-1700.

Sooryanarayama, S. (1995). Floods in Malaysia: Patterns and implications. Malaysian Journal of Tropical Ecology, 26: 35-46. 
Soulé, M. E. (1985). What is conservation biology? BioScience, 35: 727-734.

SPSS. (2009). PASW Statistics for Windows, Version 18.0. Chicago: SPSS Inc.

Staub, C. G., Binford, M. W., \& Stevens, F. R. (2013). Elephant herbivory in Majete Wildlife Reserve, Malawi. African Journal of Ecology, 51: 536-543.

Steinheim, G., Wegge, P., Fjellstad, J. I., Jnawali, S. R., \& Weladji, R. B. (2005). Dry season diets and habitat use of sympatric Asian elephants (Elephas maximus) and greater one-horned rhinoceros (Rhinocerus unicornis) in Nepal. Journal of Zoology, 265: 377-385.

Stephens, D. W., \& Krebs, J. R. (1986). Foraging theory. Princeton: Princeton University Press.

Stokke, S., \& du Toit, J. T. (2002). Sexual segregation in habitat use by elephants in Chobe National Park, Botswana. African Journal of Ecology, 40: 360-371.

Stout, J. C., \& Goulson, D. (2002). The influence of nectar secretion rates on the responses of bumblebees (Bombus spp.) to previously visited flowers. Behavioural Ecology and Sociobiology, 52: 239-246.

Sukumar, R. (1989). Ecology of the Asian elephant in Southern India 1. Movement and habitat utilisation patterns. Journal of Tropical Ecology, 5: 1-18.

Sukumar, R. (1990). Ecology of the Asian elephant in southern India. II. Feeding habits and crop raiding patterns. Journal of Tropical Ecology, 6: 33-53.

Sukumar, R., \& Ramesh, R. (1992). Stable carbon isotope ratios in Asian elephant collagen: implications for dietary studies. Stable carbon isotope ratios in Asian elephant collagen: implications for dietary studies. Oecologia, 91: 536-539.

Sukumar, R. (2003). The living elephants: evolutionary ecology, behaviour, and conservation. Oxford University Press. 
Switzer, P.V. (1993). Site fidelity in predictable and unpredictable habitats. Evolutionary Ecology, 7: 533-555.

Switzer, P. V. (1997). Factors affecting site fidelity in a territorial animal (Perithemis tenera). Animal Behaviour, 53: 865-877.

Tchamba, M. N., \& Seme, P. M. (1993). Diet and feeding behaviour of the forest elephant in the Santchou Reserve, Cameroon. African Journal of Ecology, 31: 165-171.

Theuerkauf, J., Ellenberg, H., Waitkuwait, W. E., \& Mühlenberg, M. (2001). Forest elephant distribution and habitat use in the Bossematié Forest Reserve, Ivory Coast. Pachyderm, 30: $37-43$.

Thomas, D. L., \& Taylor, E. J. (1990). Study designs and tests for comparing resource use and availability. The Journal of Wildlife Management, 54: 322-330.

Thomson, J.D., Maddison, W.P., \& Plowright, R.C. (1982). Behaviour of bumble bee pollinators of Aralia hispida Vent (Araliaceae). Oecologia, 54: 326-336.

Thomson, J. D., Peterson, S. C., \& Harder, L. D. (1987). Response of traplining bumble bees to competition experiments: Shifts in feeding location and efficiency. Oecologia, 71: 295300 .

Thomson, J. D. (1996). Trapline foraging by bumblebees: I. Persistence of flight-path geometry. Behavioural Ecology, 7: 158-164.

Tiebout, H. M. III. (1991). Daytime energy management by tropical hummingbirds: Responses to foraging constraint. Ecology, 72: 839-851.

Tilman, D., \& Kareiva, P. M. (Eds.). (1997). Spatial ecology: the role of space in population dynamics and interspecific interactions (Vol. 30). Princeton University Press.

Turchin, P. (1991). Translating foraging movements in heterogeneous environments into the spatial distribution of foragers. Ecology, 72: 1253-1266. 
Turchin, P. (1998). Quantitative analysis of movement: measuring and modeling population redistribution in animals and plants (Vol. 1). Sunderland, Massachusetts, USA: Sinauer Associates.

Valeix, M., Fritz, H., Chamaillé-Jammes, S., Bourgarel, M., \& Murindagomo, F. (2008). Fluctuations in abundance of large herbivore populations: insights into the influence of dry season rainfall and elephant numbers from long-term data. Animal conservation, 11: 391-400.

Van Aarde, R. J., \& Jackson, T. P. (2007). Megaparks for metapopulations: addressing the causes of locally high elephant numbers in southern Africa. Biological Conservation, 134: 289-297.

Vaz, J. (1997). The Kinabatangan Floodplain - An Introduction. WWF Malaysia. 64pp.

Vivas, H. J., Saether, B. E., \& Anderson, R. (1991). Optimal twig size selection of a generalist herbivore, the moose Alces alces: implications for plant-herbivore interactions. Journal of Animal Ecology, 60: 395-408.

Wall, J., Wittemyer, G., Klinkenberg, B., LeMay, V., \& Douglas-Hamilton, I. (2013). Characterizing properties and drivers of long distance movements by elephants (Loxodonta africana) in the Gourma, Mali. Biological Conservation, 157: 60-68.

Watts, D. P. (1998). Long-term habitat use by mountain gorillas Gorilla gorilla beringei. 2. Reuse of foraging areas in relation to resource abundance, quality, and depletion. International Journal of Primatology, 19: 681-702.

Web of Science (http://thomsonreuters.com/thomson-reuters-web-of-science)

Webber, C. E., Sereivathana, T., Maltby, M. P., \& Lee, P. C. (2011). Elephant crop-raiding and human-elephant conflict in Cambodia: Crop selection and seasonal timings of raids. Oryx, 45: 243-251. 
White, R. G. (1983). Foraging patterns and their multiplier effects on productivity of northern ungulates. Oikos, 40: 377-384.

White. G. C., \& Garrott. R. A. (1990). Analysis of wildlife radiotracking data. Academic Press. New York.

White, P. S., \& Harrod, J. (1997). Disturbance and diversity in a landscape context. In Wildlife and Landscape Ecology. Springer New York. pp. 128-159.

Williams, N. M., \& Thomson, J. D. (1998). Trapline foraging by bumble bees: III. Temporal patterns of visitation and foraging success at single plants. Behavioural Ecology, 9: 612-621.

Williamson, B. R. (1975). Seasonal distribution of elephant in Wankie National Park. Arnoldia, 7: 1-16.

Wilson, S. L., \& Kerley, G. L. H. (2003). Bite diameter selection by thicket browsers: the effect of body size and plant morphology on forage intake and quality. Forest Ecology Management, 181: 51-65.

Wilson, G., Desai, A. A., Sim, D. A., \& Linklater, W. L. (2013). The influence of the invasive weed Lantana camara on elephant habitat use in Mudumalai Tiger Reserve, southern India. Journal of Tropical Ecology, 29: 199-207.

Wing, L. D., \& Buss, I. O. (1970). Elephants and forest. Wildlife Monographs, 19: 1-92.

Winnie, J. A., Cross, P., \& Getz, W. (2008). Habitat quality and heterogeneity influence distribution and behaviour in African buffalo (Syncerus caffer). Ecology, 89: 1457-146. 
Wittmer, H. U., McLellan, B. N., \& Hovey, F. W. (2006). Factors influencing variation in site fidelity of woodland caribou (Rangifer tarandus caribou) in southeastern British Columbia. Canadian Journal of Zoology, 84: 537-545.

Wolf, J. B., \& Trillmich, F. (2007). Beyond habitat requirements: individual fine-scale site fidelity in a colony of the Galapagos sea lion (Zalophus wollebaeki) creates conditions for social structuring. Oecologia, 152: 553-567.

Wolf, M., Frair, J., Merrill, E., \& Turchin, P. (2009). The attraction of the known: the importance of spatial familiarity in habitat selection in wapiti Cervus elaphus. Ecography, 32: $401-410$.

Woods, P. (1989). Effects of Logging, Drought, and Fire on Structure and Composition of Tropical Forests in Sabah, Malaysia. Biotropica, 21: 291-298.

Ydenberg, R. C., \& Prins, H. T. (1981). Spring grazing and the manipulation of food quality by barnacle geese. Journal of Applied Ecology, 18: 443-453.

Zollner, P. A., \& Lima, S. L. (1999). Search strategies for landscape-level interpatch movements. Ecology, 80: 1019-1030. 